\title{
Nonlinear, dispersive, shallow-water waves developed by a moving bed
}

\author{
By Marc Villeneuve
}

Department of Civil Engineering \& Applied Mechanic's McGill University, Montreal

July 1989

A Thesis submitted to the Faculty of Graduate Studiess and Research in partial fulfillment of the requirements for the degren of Master in Engineering. 


\section{Abstract}

Landslides and avalanches plunging into lakes or reservoirs located in momtanous regions can generate large waves which can result in loss of life and significant property damage. As is the case with tsumamis generated in the ocean by molew water seismic activity, landslide induced water waves result from the motom of a solid boundary of the fluid. The present study deals with the mathemationl modelling of water waves developed in a channel by a moving bed. A set of depth-averiged governing equations is derived to predict the evolution of the free sunfuce resulting from a predetermined bed motion. These efuations, whech constitute a generalisation of the Boussinesq system for waves over a Hat bed, include both nonlinear and dispersive effects. Numerical solutions are obtained by using the finte difference method coupled with a Flux Corrected Transport (FCT) algorithm. 'The resultung model is used to predict the waves resulting from simple bed motions. The nume' ical results show an excellent agreement with corresponding "xperimental icsults obtained by .J. Sander at ETH, Zirich.

\section{Sommaire}

En région montagneuse, les glisscments de terrain et avalanche's plongeant dans les lacs ou réservoirs peuvent provoruer des ondes de forte amplitude constatuant. une menace pour les populations et les popriétés avoisinantes. Tout comme less tsunamis provocqués dans l'océan par nne activité sismique sons-marine, les ondes induites dans leau par des glissements dr terrain résultont d'un monvement. d'une des frontières solides du fluide La présrnte étude ponte sur la modólisition mathématique d'intumescences engendrées dans un canal pair nu mouvement, du fond. Des équations intégrées par rapport à la profondeur sont diveloppóés en vur

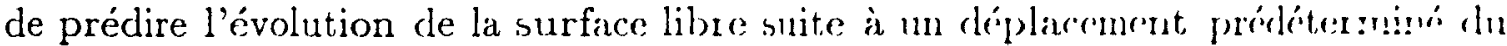
lit. Ces équations constituent une généralisation de la formulation do Bonssinesq s'appliquant aux ondes propägées sur un fond plat Elles incluent is lis fois des effotts non-linéaires et de dispersion. Des solutions numériquess sont obtermuses i l'aide rle la méthode des différences finies associée à un algonithme de 'Flux Correctcol Transport' (FCT). Le modele résultant est utilisé ponr prédne les ondres engendrán pan

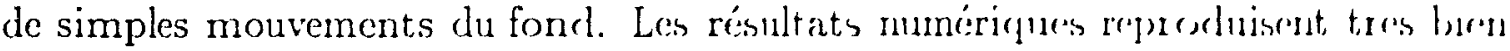
des résultats expérimentaux correspondants obtenus par J. Sinde. à ETH, Zauirh. 


\section{Acknowledgements}

The author is grateful to Dr. Stuart B. Savage for his help and guidance and to the Natural Sciences and Engineering Research Council of Canada (NSERC) for financial support of his work He also wishes to thank Dr. K. Hutter and J. Sander of the Laboratory of Hydraulics, Hydrology and Glaciology (VAW) of the Swiss Federal Institute of 'Technology (ETH) in Zürich for their kind help and cooperation and the Director Di D. Vischer for permitting the unpublished results of J. Sander to be quoted in this thesis. 


\section{Contents}

Abstract ........................

Acknowledgements .................... .

List of Figures . ...................

List of Tables . . . . . . . . . . . . . . . . . . n

1 Introduction 1

1.1 Theoretical background . . . . . . . . . . . . . 1

1.2 Thesis' outline . . . . . . . . . . . . . . . . i

2 Governing equations 10

2.1 Derivation of the governing equations . . . . . . . . . . . 10

2.2 Scaling of the rning equations . . . . . . . . . . . 15)

3 Numerical solutions $\quad 21$

3.1 The finte difference method . . . . . . . . . . . . . . . 21

31.1 Eulerian description ... ............... 22

31.2 Lagrangian description . . . . . . . . . . . . 21

3.1 .3 Explicit and implecit formulations . . . . . . . . 24;

32 Finte difference formulation of the geverning equations . . . . 28

3.2.1 Chose of the flow field description . . . . . . . . 28

32.2 Eulerian schrme . . . . . . . . . . . . 29)

3.2.3 Lagrangian scheme . . . . . . . . . . . . 32

3.3 Initial and boundary conditions . . . . . . . . . . . . 34

3.3 .1 Initial conditions . . . . . . . . . . . . . . 34

3.3.2 Boundary conditions ... . . . . . . . . . . . 35)

3.4 The Flux Corrected Transpont (FCT) methorl _. . . . .

4 Computations and experiments 41

41 Types of bed motion considened . . . . . . . . . H

4.1.1 Moving wall . . . . . . . . . . . . . . H2

412 Submerged wedge . . . . . . . . . . . . . . . . . . . . . .

4.1.3 Moving shelf . . . . . . . . . . . . 11

4.1.4 Rotating plate .............. . . . 4

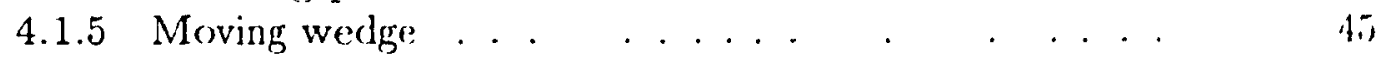

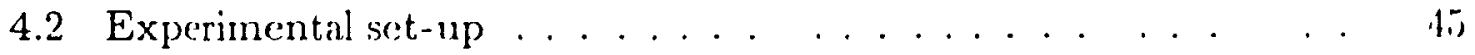

4.2.1 Rotating platc . . . . . . . . . . . . . 4.)

4.2.2 Lateral displacements $\quad \ldots . . . . . . .446$

4.3 Computer models . . . . . $\quad$. . . . . . . . . . 47

4.3.1 General description ... . . . . . . . . . . 17

4.3.2 Program WALL . . . . . . . . . . . . . . 49

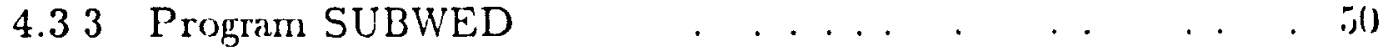

4.3.4 Program SHELF . . . . . . . . . . . . . . . 51 


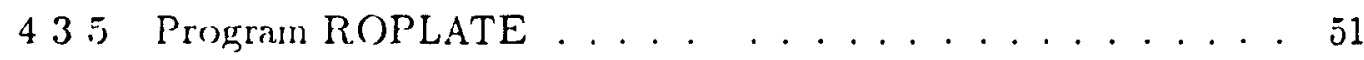

4.3.6 Program WEDGE ................ 51

5 Comparison of results 53

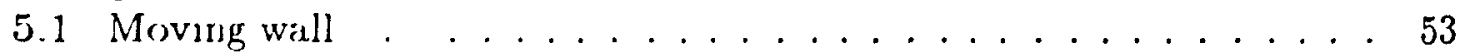

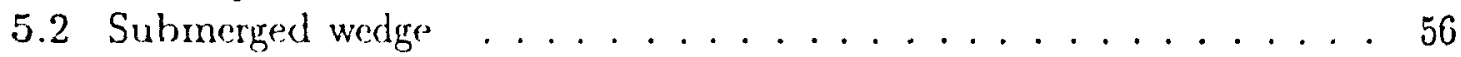

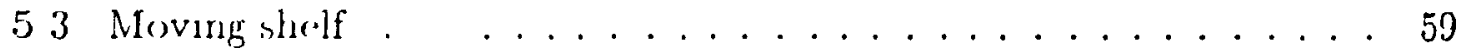

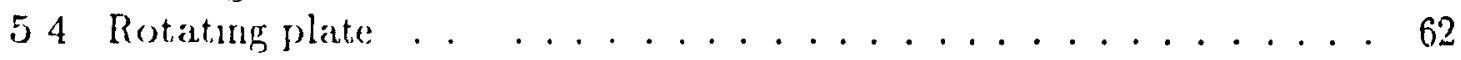

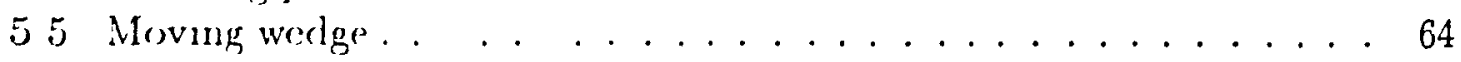

6 Conclusions $\quad 67$

$\begin{array}{ll}\text { References } & 69\end{array}$

\section{A ppendices}

A Validity of the shallow-water approximation .............A-1

B Derivation of the governing equations using an expansion method . . B-1

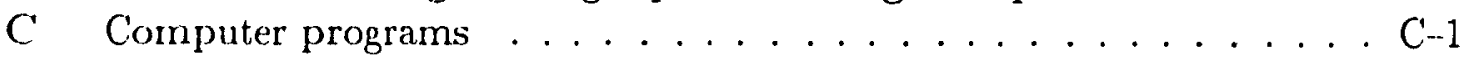

D Supplementary results ..................... D 


\section{List of Figures}

2.1 Definition sketch for waves generated by a moving bed. . . . . 10

31 The Euler an finite difference problem. . . . . . . . . . 22

3.2 The Lagrangian finite difference problem. . . . . . . . . . 24

33 The Lagrangian grid. . . . . . . . . . . . . . . . 25

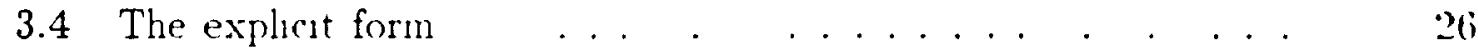

3.5 The fully implicit form . . . . . . . . . . . . .

3.6 The Crank-Nicolson form . . . . . . . . . . . 27

3.7 Eulerian description of the flow field . . . . . . . . . 29)

3.8 Lagrangan description of the flow field. . . . . . . . . . 33

4.1 Types of bed motion consudered. . . . . . . . . . . . 43

5.1 a Wave generated by a moving wall. . . . . . . . . . . 5.3

$5.1 \mathrm{~b}$ Wave generated by a moving wall (comparison with experment) . 5i)

5.2 a Wave generated by a submerged wedge. . . . . . . . . . 5 (j)

$5.2 \mathrm{~b}$ Wave generated by a submerged wedge (comparison with experiment) 58

5.3 a Wave generated by a moving shelf. . . . . . . . . . . 60

5.3b Wave generated by a moving shelf (comparison with expermuent). 61

$5.4 \mathrm{a}$ Wave generated by a rotating plate ... . . . . $\quad 62$

$5.4 b$ Wave generated by a rotating plate (comparison with expeninent)

55 a Wave generated by a moving werlge . . . . . . (5;)

$5.5 \mathrm{~b}$ Wave generated by a moving wedge (comparison with expernnent) (bif 


\section{List of Tables}

41 Types of bed motion considered and corresponding computer programs 48 


\section{INTRODUCTION}

\subsection{Theoretical background}

In mountainous regions, rockslides, snow or ice avalanches and calving glacie'rs somutimes enter lakes and water supply reservoirs. In doing so they often generate lange waves which can result in loss of life and significant property damage. One of the greatest disasters of that type occured in the Vaiont Valley, Italy in 1963 where a wave generated by a landslide into a reservoir completely destroyed a town and clanmel over 2,000 lives. Similar events dating to, at least, 1731 also occured m many fouds of Norway and caused hundreds of fatalities. Among other well known examples ald the 1792 catastrophe of Shimbara Bay, Japan; the 1850 and 1905 glacier falls of D) enchantment Bay and the 1958 rockslide of Lituya Bay, Alaskin, and findly the 1971 landslide in Lake Yan. inuin, Peru. The combined death toll from all these disisters exceeds 20,000 people (Slingerland \& Voight 1979). Huber (1982) has given dultulh of five rock avalanches and a bankslide that occured in Swiss lakes between 1!)'! and 1974; all resulted in serious damage to the shoreline structures.

Landslide generated waves in lakes and reservoirs have nuch in common will tsunamis which are oceanic waves resulting from underwater seismic activity (nee Voit (1987) for a recent review of tsunamis). Three stages of development are usullly associated with both of these phenomena:

1. Generation of an initial wave by a motion of the fluid's boundary

2. Free propagation in water of approximately constant dopth.

3. Propagation of the wave in coastal waters of nonuniform depth where the shal lowness results in amplification and strong deformation of the wave profile pmos to the runup on beaches.

One of the important problems arising in the theoretical treatment, of the wo then. stages is the choice of approximations to obtain appropriate mathematical morlels 
Peregrine (1972) reviews important water wave equations and their underlying approximations.

The equations of the linear (or 'small-amplitude') theory are obtained by linearization of the free surface boundary conditions; they are only valid for waves propagating in water having a depth of the same crder as the wavelength (i.e. deep water waves) and an amplitude negligible compared to the water depth (i.e. smallamplitude waves). These waves have a tendency to spread out as they propagate, an effect known as frequency dispersion. In one spatial dimension, if we denote the resper tive positions of the freesurface and the bed by $\eta$ and $h$, we can use the velocity potentual $\phi$ to write :

$$
\begin{gathered}
\nabla^{2} \phi=0 \\
\eta_{t}=\phi_{y} \text { and } \phi_{t}+g \eta=0 \text { on } y=0 \\
\phi_{x} h_{x}+\phi_{y}=0 \text { on } y=-h(x)
\end{gathered}
$$

If on the other hand we only know that the water depth to wavelength ratio is small (i ' shallow-water, or 'long' waves), it can be assumed that the pressure distribution is hydrostatic or equivalently that the horizontal velocity is uniform with depth. The resulting nonlinear equations form the basis of the 'finite-amplitude, shallow-water theory':

$$
\begin{aligned}
& \eta_{t}+[(h+\eta) \bar{u}]_{x}=0 \\
& \bar{u}_{t}+\overline{u u}_{x}+g \eta_{x}=0
\end{aligned}
$$

where $\bar{u}$ represents the uniform horizontal velocity of the fluid. Waves predicted by that theory steepen as they propagate under the influence of nonlinearity: this phenomenon is sometimes called amplitude dispersion.

If in addition to the shallow-water approximation, it is assumed that the amplitude to water depth ratio is of small (but not negligible) order, equations can be derived In which the nonlinear effect of amplitude dispersion is approximately balanced by the linear effect of frequency dispersion. The resulting weakly nonlinear. dispersive "quations are called the Boussinesq equations. The typical solution of these equations 
is the solitary wave which is a single wave of elevation propagating without change in form. For waves propagating in water of unit depth, the Boussinesq system (Witham 1974) is

$$
\begin{gathered}
\eta_{t}+\left[(h+\eta ! \bar{u}]_{\Gamma}=0\right. \\
\bar{u}_{t}+\overline{u u}_{x}+g \eta_{x}=\frac{1}{3} \bar{u}_{x x t}
\end{gathered}
$$

Finally, the neglect of both the nonlinear and dispersive terms in the above the. ories leads to the 'linearized long-wave theory':

$$
\begin{gathered}
\eta_{t}+(h \bar{u})_{x}=0 \\
\bar{u}_{t}+g \eta_{x}=0 .
\end{gathered}
$$

In the following text, the term 'linear theory' will only refer to the linearrzed, small. amplitude equations and not to the linearized long-wave equations.

Since they represent the most direct threat to shoreline populations and strurtures, the shoaling and runup of landslide generated waves and tsunams have teceiverl much attention This third stage of development involves waves in shallow water and clearly requires a nonlinear theory. In most cases, the incoming wave in mostelled as a solitary wave propagating over a nonuniform (usually uniformly slopmung) bed. Weakly nonlinear, dispersive equations were derived by Peregrine (19667). 'Thesse depth-averaged equations are a generalization of the Boussinesq system for waven over a flat bed. Higher order equations have also been derived (Seabras-fiantos, Renonaud \& Temperville 1987).

As it approaches the shoreline, the wave front steepens under the mflucuce of shallowness. The treatment of the actual runup depends on the wave chardeteristirs and on the beach geometry. If the wave slope is small-as it is for most tsunimul: and landslide generated waves-or if the beach slope is large, the wave: will not have a tendency to break. If the beach slope steepness does not exceed a certain limit, the runup can then be described analytically by uniformly valid solutions of the finiteamplitude, shallow-water (Airy) equations (Carrier \& Greenspan 1958, Spielvogrel 
1975). Otherwise, it can be modelled by numerical solutions of equations of the Boussinesq type (Pedersen \& Gjevik 1983). For steeper wave slopes or for very mild beach slopes, the incoming wave will break. Peregrine (1983) reviews the case of breaking waves.

The linear theory and the Boussinesq system have both been used to model the frec propagation stage in water of approximately constant depth. The respective validity of these models is highly dependent on the characteristics of the initially generated waves and on the water depth of the region in which they subsequently propragate.

The choice of an appropriate model for the generation stage still remains an open question. Until recently, before the availability of numerical solutions, the linear theory was almost the inevitable choice. Therefore, it had to be assumed that the amplitude of the generated wave was very small relative to both the water depth and the wavelength. The generation of the wave was often represented as an initial disturbance (clevation or depression) of the free surface imposed at the end of a constant depth channel (Kranzer \& Keller 1959, Wiegel et al. 1970, Noda 1971). That initial condition is equivalent to the vertically falling box model used by Noda (1970) to simulate a vertical rockfall into a channel or to the impulsive upthrust of a bed's segment used by Hammack (1973) in the study of tsunami generation. In all cases, the resulting theoretical problem was similar to the well known Cauchy-Poisson problem (Wehausen \& Laitonen 1960). Mc"e recently, Hunt (19SS) used the linear theory to model landslide generated waves by injecting an instantaneous point source of flud through the bottom of a channel; his results were very close to those of Noda (1970). Solutions of the linear theory ate integrals which are obtained by various transform methods. These integrals are usually very difficult to evaluate numerically due to the oscillatory nature of the integrands and the usual procedure is to apply asymptotic methods (stationary phase and steepest descent) which yield solutions which are valid only for large time and large distances from the source of generation.

Wiegel et al. (1970) compared their solutions with the experiments of Prins (1958) 
and also performed their own experiments to simulate vertical rochfalls by diopping vertically falling boxes at the enc of a channel. Noda (1970) compared his theoretical results with these box dop experiments. In both cases, it was found that the lincil theory was only valid for small height and length of the mitial distubance / Wieger af al. 1970) or equivalently for small length and vertical displacements of the falling hox (Noda 1970). For larger dimensions, nonlmear effects became noticeable as solitury waves and bores started to develop. Noda (1970) also simulated horizontal landsidess by the horizontal translation of a vertical wall. The corresponding experiments of Das \& Wiegel (1972) showed the results of the linear theory to be only valid for small displacements of the wall. The above conclusions were to be expected since the linean theory is only applicable in the case of small-amplitude waves which in turn can only be generated by small displacements or dimensions of the moving boundaries.

However, since most of the above theoretiral results were only valid for lang" values of time and distance, it was not possible to determine if the linedu theny only became invalid in the free propagation stage or right from the stait of the generation. Hammack (1973) looked at this problem in the context of tsmunmis generated by a positive or negative vertical displacement of a segment of the berl. He considered separately the ranges of validity of the linear theory in the generotion and propagation stages. By scaling the fundamental equations of motion, he fomm that, for impulsive bed motions, the linear theory was only correct for generalion by very small bed displacements. For slow bed motions, he showed the linear theory to be valid for arbitrary large bed displacements. Using Ursell number considerations, he also claimed that the Boussinesq system should be preferred to the linear theresy for the free propagation stage during which nonlinear effects accumulate? with tume The Ursell number is a dimensionless number measuring the relative mportiur. of nonlinear to linear wave effects (Ursell 1953).

From the above, it appears that one of the main disadvantages of the lincar thersy is that it only possesses narrow ranges of validity and thus can only describe very limited generating conditions. Under the influence of frequency dispersion, waves 
generated and propagated using the linear theory eventually become independent of the geometry and time history of the generating process and only depend on the volume of water displaced originally (Hunt 1988). That description may account for waves created by rapid rockfalls of small dimensions but certainly does not describe properly laige progressive bankslides in which the generated wave often propagates without change in shape, almost as a solitary wave (Huber 1982). Furthermore, the complexity of the integral solutions even for simple bed motions precludes the application of the linear theory to more realistic descriptions of the moving bed. Sabatier (1983) used the linear theory for waves generated by ground motions on a slope.

One of the first attempts to use numerical solutions of nonlinear wave theories In the description of the generation stage was made by Hwang \& Divoky (1970). They used the finite-amplitude, shallow-water equations in two spatial dimensions to model the tsunami which resulted from the Alaskan Good Friday earthquake of 1964. The effect of the bed motion was incorporated directly in the depth-averaged continuity equation. In that respect the numerical treatment of the governing equations possesses the advantage of allowing one to treat nonlinearity and more complex bed motions with little increase in difficulty over that corresponding to the use of the linear wave equations. That is of considerable importance since, as pointed out by II wang \& Divoky (1970), one of the fundamental problems in the generation phase is the accurate estimation of the bed motion, not only in its permanent displacement (as implied by impulsive linear theories), but also in its complicated time history. Comparison of their numerical results with limited field data showed an encouraging agreement.

Rancy \& Butler (1976) used a similar procedure to study landslide generated waves but modified the governing equations to include forcing functions accounting for the volume displacement, viscous drag and "form" drag exerted by a landslide on the water. Their numerical solutions were only presumed good for the leading wave snce their governing equations did not properly account for wave rumup and 
reflection.

The finite-amplitude, shallow-water theory has the advantage over the linear the. ory of allowing for the propagation of 'arger amplitude waves. However, it can harlly' be used in the free propagation stage since it ignores frequency dispersion and ult. mately leads to an infinite 'wave steepuess. Therefore, as with the case of the lum theory, the nonlinear nondispersive theory suffers from a narrow range of applicability:

The same can also be said about the linearized long-wave equations (Tuch \&. Hwang 1972) which can be used to some extent in the generation stage but raprdly become invalid.

The logical alternative to the above theories is to assume that the amplitude to depth ratio is small and derive model equations which combine the linear efrect of frequency dispersion and the nonlinear effect of amplitude dispersion. Wu (19si) derived weakly nonlinear, dispersive (Boussinesq) equations including the elfer ts of the moving bed. These equations would appear to constitute a very good model tor the complete description of the generation and propagation stages. Unfortumalcly, Wu did not present numerical results for actual moving bed problems.

\subsection{Thesis' outline}

The following thesis is concerned with the mathematical modelling of water waves developed by a moving bed. It was performed at McCill University in conjunctimn with corresponding experimental work carried out at the Laboratory of Hydiaulis, Hydrology and Glaciology directed by Dr. Daniel Vischer and affiliated to the Siwish Federal Institute of Technology in Zürich. The experiments were made by Johamnus Sander under the direction of Drs. Kolumban IIutter and Daniel Vischer.

The basic idea was to check the validity of a depth-averaged, weakly nomlunin and dispersive (i.e. Boussinesq) approach to the numerical modelling of watcr waves induced by landslides and avalanches plunging into alpine lakes and water supply reservoirs. The logical starting point was to consider generation and propagation in 
one spatial dimension before studying more realistic problems in two dimensions.

The present study deals primarily with waves generated at the upstream end of a long channel. The generation of the wave by the incoming material is simulated by the motion of an impervious boundary at the end of the channel. The above schematizations lead to the development of a model which has the advantage of being applicable not only to landslide generated waves but to all problems involving the generation of water waves under the action of moving solid boundaries.

The thesis is divided in six sections. In $\S 2$, the fundamental equations governing the motion of an inviscid, incompressible and irrotational fluid aro integrated over the water depth. The resulting depth-averaged equations give the free surface disturbance $\eta(x, l)$ and the depth-averaged fluid velocity $\bar{u}(x, t)$ resulting from a known bed history $h(x, t)$. These high-order equations are a generalization of those derived by $\mathrm{Su} \&$ Gardner (1969) for waves over a steady, uniform bed and by Seabra-Santos, Renouard \&: Temperville (1987) for waves over a steady, nonuniform bed. The validity of the shallow-water assumption in problems involving a moving bed is discussed in Appendix A.

Scaling parameters pertaining to the wave characteristics are introduced in $\S 2.2$ in order to reduce the governing equations to a 'Boussinesq' formulation in which nonlinear wave effects are approximately balanced by dispersive effects. The resulting equations are identical to those derived by Wu (1981). Appendix B outlines a derivation of these equations using a more direct pertubation method.

Finite difference schemes are developed in $\S 3$ to solve the depth-averaged contimuity and momentum equations. The basic algorithm consists of a simple three step scheme in which both explicit and implicit finite difference approximations are used. The Eulerian specification of the flow field is used except in problems involving a laterally moving waterline. These problems are more easily treated with the help of the Lagrangian formulation.

Initial conditions and boundary conditions applying to the various types of bed motions are described in $\S 3.3$. A Flux Corrected Transport (FCT) algorithm is pre- 
sented in $\S 3.4$ in order to eliminate spurious oscillations from the computed free surface profiles.

Five simple moving bed geometries are modelled both numerically and expen mentally:

1. Moving vertical wall,

2. Moving submerged wedge,

3. Moving shelf,

4. Rotating plate,

5. Moving wedge.

The above bed motions are presented and described in \$1.1. Apart from the 'rotating plate' wave generating mechanism, the four remaining bed motions arise from the horizontal translation of a wavemaker having a variable geometry.

The experimental set-up used by Sander (19S8) is briefly described in $2 \mathrm{am}$ corresponding computer models are introduced in $\$ 4.3$. Appendix $\mathrm{C}$ gives the listing of the Pascal source codes of the programs.

Comparisons between the numerical and experimcntal results are treated in (35) In every cases, the agreement between computed and measured wave heights is axcollont. and confirms the validity of the weakly nonlinear, dispersive numerical model for the simulation of water waves developed by a moving bed. 


\section{GOVERNING EQUATIONS}

\subsection{Derivation of the governing equations}

We start by considering an incompressible, inviscid and irrotational fluid bounded by a free surface $y=\eta(x, t)$ and a bed $y=-h(x, t)$ with the position of the free surface at rest being $y=0$. The total water depth $h+\eta$ is denoted by $H$ (Figure 2.1). Our objective in the study of water waves developed by a moving bed consists in

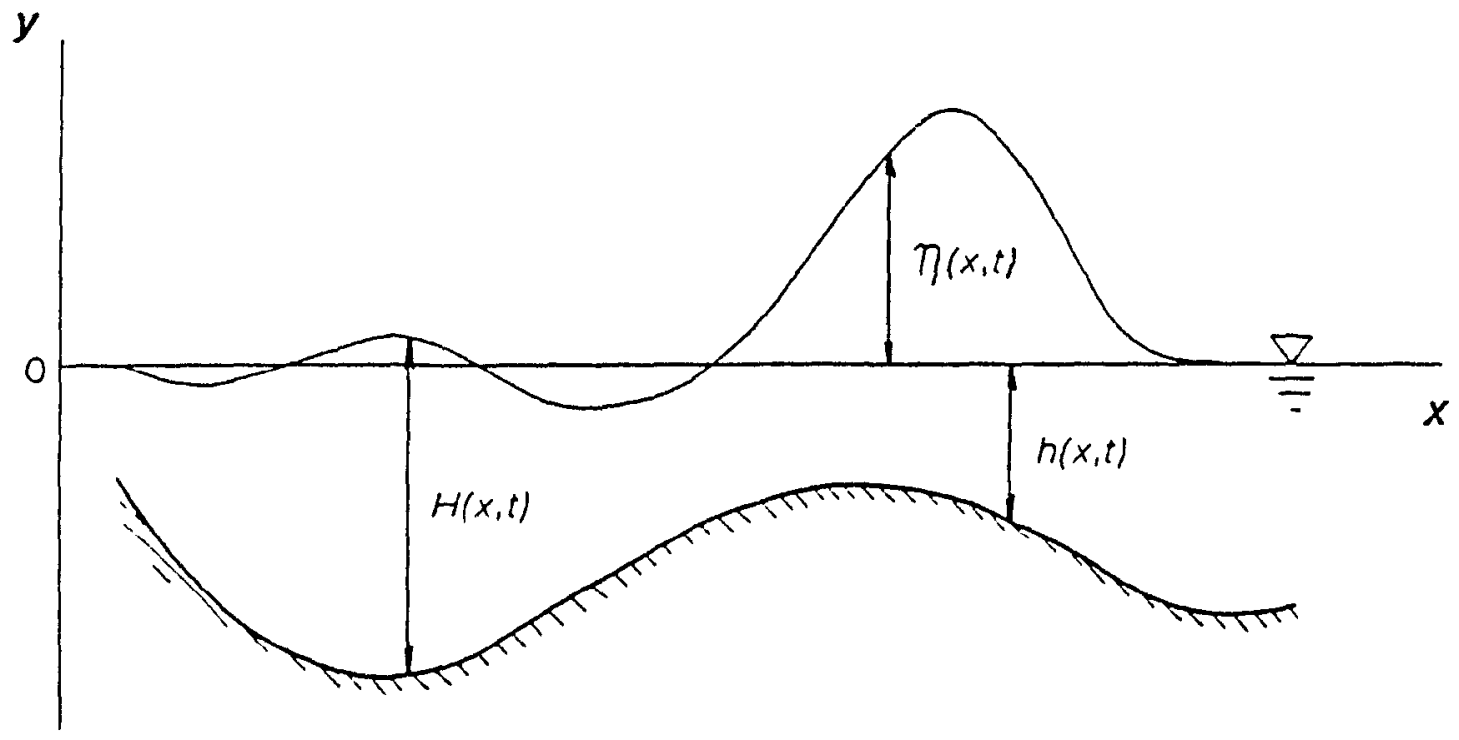

Figure 2.1: Definition sketch for waves generated by a moving bed.

determining the deformation $\eta(x, t)$ of the free surface resulting from a prescribed motion $h(x, t)$ of the bed. The following non-dimensional variables are used:

$$
\begin{aligned}
(x, y, \eta, h) & =h_{o}^{-1}\left(x^{\prime}, y^{\prime}, \eta^{\prime}, h^{\prime}\right), \\
(u, v) & =\left(g h_{o}\right)^{-\frac{1}{2}}\left(u^{\prime}, v^{\prime}\right), \\
p & =p^{\prime} /\left(\rho g h_{o}\right), \\
t & =\left(g / h_{o}\right)^{\frac{1}{2}} t^{\prime},
\end{aligned}
$$

where the primes denote dimensional (i.e. physical) variables and $h_{0}$ is a characteristic value for the water depth. The horizontal and vertical components of the fluid velocity 
are respectively $u=u(x, y, t)$ and $v=v(x, y, t)$ The pressure at any point in the llund is given by $p(x, y, t)$. The constant fluid density is $\rho$ and the gravitational acceleralum $g$ acts vertically downward. The fundamental two-dimensional equations of motion are then:

$$
\begin{gathered}
\frac{\partial u}{\partial x}+\frac{\partial v}{\partial y}=0 \\
\frac{\partial u}{\partial t}+u \frac{\partial u}{\partial x}+v \frac{\partial u}{\partial y}=-\frac{\partial p}{\partial x} \\
\frac{\partial v}{\partial t}+u \frac{\partial v}{\partial x}+v \frac{\partial v}{\partial y}=-\frac{\partial p}{\partial y}-1 \\
\frac{\partial u}{\partial y}-\frac{\partial v}{\partial x}=0
\end{gathered}
$$

Equation (2.1) is the continuity equation for an incompressible fluid. Equations (2:2) and (2.3) are Euler's momentum equations in the $x$ and $y$ directions respectively and (2.4) is the irrotationality condition. Equations (2.1)--(2.1) are subject to thire kinematic boundary conditions

$$
\begin{aligned}
& v_{s}=\frac{\partial \eta}{\partial t}+u_{s} \frac{\partial \eta}{\partial x} \\
& v_{b}=-\frac{\partial h}{\partial t}-u_{b} \frac{\partial h}{\partial x}
\end{aligned}
$$

and the stress free surface condition

$$
p_{s}=0
$$

where the subscripts $s$ and $b$ respectively indicate quantities evaluated at thr fir. surface and at the bed. The boundary conditions (2.5) and (2.6) state lliat a flurd particle located at the free surface (or at the bed) subsequently moves along with tha" free surface (or the bed). The boundary condition (2.7) simply implies that. there is no pressure acting on the free surface.

Integration of the continuity equation (2.1) in the vertical direction, from the l, red to the free surface, yields

$$
v_{s}-v_{b}=u_{s} \frac{\partial \eta}{\partial x}+u_{b} \frac{\partial h}{\partial x}-\frac{\partial}{\partial x} \int_{-h}^{\eta} u d y
$$


in whuch the kinematic boundary conditions (2.5) and (2.6) can be substituted to give

$$
\frac{\partial}{\partial t}(h+\eta)+\frac{\partial}{\partial x}[(h+\eta) \bar{u}]=0
$$

where

$$
\bar{u}(x, t)=\frac{1}{H} \int_{-h}^{\eta} u(x, y, t) d y .
$$

Equation (2.9) is the depth-averaged continuity equation. For a known bed history $h(x, t)$, it gives the evolution of the free surface $\eta$, provided we know the depthaveragerl velocity $\bar{u}$. Note that (2.9) is exact since no approximation was made in its derivation.

For shallow-water wave propagation, it is usually assumed that the horizontal velocity of the fluid is approximately uniform with depth:

$$
u(x, y, t) \simeq \bar{u}(x, t) .
$$

By mtegrating the continuity equation (2.1) using (2.11) and the kinematic boundary condition (2.6), we can obtain a corresponding approximation for the vertical fluid velocity:

$$
v(x, y, t) \simeq-\frac{\partial h}{\partial t}-\frac{\partial}{\partial x}(h \bar{u})-y \frac{\partial \bar{u}}{\partial x} .
$$

In view of deriving an evolution equation for $\bar{u}$, we substitute the approxiomation (2.11) in the $x$-momentum equation (2.2):

$$
\frac{\partial \bar{u}}{\partial t}+\bar{u} \frac{\partial \bar{u}}{\partial x}+\frac{\partial p}{\partial x}=0 .
$$

Equation (2.13) can be integrated from $y=-h$ to $\eta$ with the boundary conditions $(2.5)-(2.7)$ to give

$$
\frac{\partial}{\partial t}(H \bar{u})+\frac{\partial}{\partial x}\left[H\left(\bar{u}^{2}+\bar{p}\right)\right]=p_{b} \frac{\partial h}{\partial x}
$$

where the depth-averaged pressure is

$$
\bar{p}(x, t)=\frac{1}{H} \int_{-h}^{\eta} p(x, y, t) d y .
$$


It is important to note that the depth-averaging of the complete $x$-momentum enuntion (2.2), without the approximation (2.11), yields

$$
\frac{\partial}{\partial t}(H \bar{u})+\frac{\partial}{\partial x}\left[H\left(\bar{u}^{2}+\bar{p}\right)+H\left(\overline{u^{2}}-\bar{u}^{2}\right)\right]=p_{b} \frac{\partial h}{\partial x}
$$

with

$$
\overline{u^{2}}(x, t)=\frac{1}{H} \int_{-h}^{\eta} u^{2}(x, y, t) d y .
$$

Therefore the validity of the approximation (2.11) which led to equation $(2.14)$ implen that the term $H\left(\overline{u^{2}}-\bar{u}^{2}\right)$ is of small order compared to the remaining terms of equat ien (2.16). That requirement is always satisfied for a flat bed, but in the case of a moving bed, it imposes certain restraints on the bed slope and velocity (Appendix $A$ )

An expression for the pressure is obtained by integration of the $y$-momentum equation (2.3) with the dynamic boundary condition (2.6):

$$
p=(\eta-y)+\int_{y}^{\eta} \frac{d v}{d t} d y
$$

where

$$
\frac{d}{d t}=\frac{\partial}{\partial t}+u \frac{\partial}{\partial x}+v \frac{\partial}{\partial y}
$$

which can be approximated as:

$$
\frac{d}{d t} \simeq \frac{\partial}{\partial t}+\bar{u} \frac{\partial}{\partial x}+v \frac{\partial}{\partial y} .
$$

The second term on the right hand side of (2.18) represents the correction to the hydrostatic pressure. Using the approximation (2.12) for $v$, we can write:

$$
\frac{d v}{d t}=-\frac{d h_{t}}{d t}-\frac{d(h \bar{u})_{x}}{d t}-y \frac{d \bar{u}_{x}}{d t}-v \bar{u}_{x}
$$

or

$$
\frac{d v}{d t}=-\beta-\gamma-y \alpha
$$

where

$$
\begin{gathered}
\alpha=\frac{d \bar{u}_{x}}{d t}-\bar{u}_{x}^{2}, \\
\beta=\frac{d(h \bar{u})_{x}}{d t}-(h \bar{u})_{x} \bar{u}_{x},
\end{gathered}
$$




$$
\gamma=\frac{d h_{t}}{d t}-h_{t} \bar{u}_{x}
$$

Substutution of (2.19) in (2.18) gaves

$$
p=(\eta-y)(1-\beta-\gamma)-\left(\frac{\eta^{2}-y^{2}}{2}\right) \alpha .
$$

The pressure at the bed and the depth-averaged pressure are then:

$$
\begin{gathered}
p_{b}=H(1-\beta-\gamma)-\left(\frac{\eta^{2}-h^{2}}{2}\right) \alpha \\
\bar{p}=\frac{H}{2}(1-\beta-\gamma)-\frac{1}{H}\left(\frac{\eta^{3}}{3}+\frac{\eta^{2} h}{2}-\frac{h^{3}}{6}\right) \alpha .
\end{gathered}
$$

Finally, substitution of $(2.22)-(2.23)$ in (2.14) gives:

$$
\begin{aligned}
& \frac{\partial}{\partial t}(H \bar{u})+\frac{\partial}{\partial x}\left[H \bar{u}^{2}+\frac{H^{2}}{2}(1-\beta-\gamma)-\left(\frac{\eta^{3}}{3}+\frac{\eta^{2} h}{2}-\frac{h^{3}}{6}\right) \alpha\right] \\
& =\frac{\partial h}{\partial x}\left[H(1-\beta-\gamma)-\left(\frac{\eta^{2}-h^{2}}{2}\right) \alpha\right] \text {. }
\end{aligned}
$$

Lifuation (2.24) togethes with the contmusty equation (2.9) constitute a set of depthaveraged equations governing the amplitude $\eta$ and velocity $\bar{u}$ of waves developed by amoving bed.

If the bed is not moving, $h_{t}=0$ and (2.24) can be simplified. The term $\gamma$ defined by $(220 c)$ vanishes and $\beta$ in (2.20b) reduces to:

$$
\beta=\bar{u}_{t} h_{x}+\bar{u}^{2} h_{x x}+\overline{u u}_{x} h_{x}+h \alpha .
$$

The resulting equation is then:

$$
\frac{\partial}{\partial t}(H \bar{u})+\frac{\partial}{\partial x}\left[H \bar{u}^{2}+\frac{H^{2}}{2}(1-\phi)-\frac{H^{3}}{3} \alpha\right]=\frac{\partial h}{\partial x}\left[H(1-\phi)-\frac{H^{2}}{2} \alpha\right]
$$

where

$$
\phi=\beta-h \alpha=\bar{u}_{t} h_{x}+\overline{u u}_{x} h_{x}+\bar{u}^{2} h_{x x}
$$

Equation (2.25) is the same as the equation derived by Seabra-Santos, Renouard \& 'Temperville (198T). 
For waves over a flat, horizontal bed, $\gamma$ is again zero and since $h_{x}=0$, we haw $\beta=h \alpha$. The governing equation (2. 24) becomes

$$
\frac{\partial}{\partial t}(H \bar{u})+\frac{\partial}{\partial x}\left(H \bar{u}^{2}+\frac{H^{2}}{2}-\frac{H^{3}}{3} \alpha\right)=0
$$

which is identical to the "correction" equation to the shallow-water theory demend by Su \& Gardner (1969).

\subsection{Scaling of the governing equations}

Compared with corresponding 'Boussinesq' formulations, the governung colustium (2.24)-(2.26) derived in the previous section include extra terms which ase of higher order. However, for most practical problems, these terms are so small that they can be expected to have little or no effect on the resulting solutions. If appropulate scaling parameters are introduced to represent the respective order of magmutude of the problem's variables, these higher order terms can be identified and climinulled Such a procedure not only gives insight into the relative importance of the valums terms in the governing equations but also facilitates the computations of numenual solutions by greatly reducing the length of the equations that have to be progt ammened

Two scaling factors are usually associated with water wave theorles. 'The walle depth to wavelength ratio is represented by $\sigma$ while $\epsilon$ denotes the wave ampliturle to water depth ratio:

$$
\begin{aligned}
& \sigma=\frac{h_{o}}{\lambda}, \\
& \epsilon=\frac{a}{h_{o}} .
\end{aligned}
$$

The relative size of these parameters is incorporated in the well known Ursell mumb's

$$
U r=\frac{\epsilon}{\sigma^{2}}=\frac{a \lambda^{2}}{h_{o}^{3}}
$$

which measures the importance of nonlinear to linear effects in wave propagation (Ursell 19.53). Thus $U r \ll 1$ for linear waves, while $U r \gg 1$ for fully nonlincaar (Aury) waves. Our interest lies in weakly nonlinear, dispersive (Boussinesq) waves for which $U r \sim 1$. We therefore assume $\epsilon \sim \sigma^{2}$ in what follows. 
For all types of long waves, since the variations of $x$ and $t$ are both small in terms of the wavelength, we can write

$$
(x, t)=\frac{1}{\sigma}\left(x^{*}, t^{*}\right)
$$

where the asterisks denote non-dimensional, scaled variables. The relation (2.27a) implies that the change in the scaled variables is of $O(1)$ for a significant change in the: wave. Furthermore, weakly nonlinear, dispersive, shallow-water waves also have a small amplitude to depth ratio. The appropriate scaling for $\eta$ and $\bar{u}$ is then

$$
(\eta, \bar{u})=c\left(\eta^{*}, \bar{u}^{*}\right)
$$

which states that a small change in wave amplitude or in depth-averaged velocity becomes $O(1)$ in the scaled variables.

In the case of a moving bed, scaling factors can also be determined for the bed velocity $h_{t}$ and slope $h_{x}$. Clearly, since $\eta=O(\epsilon)$ and $h=O(1)$, we have $H=O(1)$. Equation (2.27) can be substituted in the depth-averaged continuity equation (2.9) lo give.

$$
\frac{\partial h}{\partial t}+c\left(\bar{u}^{*} \frac{\partial h}{\partial x}+\sigma h \frac{\partial \bar{u}^{*}}{\partial x^{*}}\right)+\epsilon^{2} \sigma\left(\bar{u}^{*} \frac{\partial \eta^{*}}{\partial x^{*}}+\eta^{*} \frac{\partial \bar{u}^{*}}{\partial x^{*}}\right)=0 .
$$

for the above equation to be consistent, we will therefore impose

$$
\frac{\partial h}{\partial t}=\epsilon \sigma\left(h_{t}\right)^{*}
$$

and

$$
\frac{\partial h}{\partial x}=\sigma\left(h_{x}\right)^{*}
$$

which leads to the following non-diniensional, scaled depth-averaged continuity equalion:

$$
\left(h_{t}\right)^{*}+\frac{\partial \eta^{*}}{\partial t^{*}}+h \frac{\partial \bar{u}^{*}}{\partial x^{*}}+\bar{u}^{*}\left(h_{x}\right)^{*}+\epsilon\left(\bar{u}^{*} \frac{\partial \eta^{*}}{\partial x^{*}}+\eta^{*} \frac{\partial \bar{u}^{*}}{\partial x^{*}}\right)=0 .
$$

With the help of the continuity equation (2.9), the momentum equation (2.24) can be learranged as :

$$
H\left(\frac{\partial \bar{u}}{\partial t}+\bar{u} \frac{\partial \bar{u}}{\partial x}\right)=H \frac{\partial H}{\partial x}(-1+\beta+\gamma)+\frac{H^{2}}{2} \frac{\partial}{\partial x}(\beta+\gamma)
$$




$$
\begin{aligned}
& +\left[\frac{\partial}{\partial x}\left(\frac{\eta^{3}}{3}+\frac{\eta^{2} h}{2}-\frac{h^{3}}{6}\right)-\frac{\partial h}{\partial x}\left(\frac{\eta^{2}-h^{2}}{2}\right)\right] \alpha \\
& +\left(\frac{\eta^{3}}{3}+\frac{\eta^{2} h}{2}-\frac{h^{3}}{6}\right) \frac{\partial \alpha}{\partial x}+\frac{\partial h}{\partial x}[H(1-\beta-\gamma)]
\end{aligned}
$$

which simplifies to

$$
\begin{aligned}
\frac{\partial \bar{u}}{\partial t}+\bar{u} \frac{\partial \bar{u}}{\partial x}+\frac{\partial \eta}{\partial x}= & \frac{H}{2} \frac{\partial}{\partial x}(\beta+\gamma)+\frac{1}{H}\left(\frac{\eta^{3}}{3}+\frac{\eta^{2} h}{2}-\frac{h^{3}}{6}\right) \frac{\partial \alpha}{\partial x} \\
& +\frac{\partial \eta}{\partial x}(\eta \alpha+\beta+\alpha) .
\end{aligned}
$$

The terms $\alpha, \beta$ and $\gamma$ defined by (2.20) can be scaled as follows :

$$
\begin{gathered}
\alpha^{*}=\left(\epsilon \sigma^{2}\right) \bar{u}_{x^{*} t^{*}}^{*}+\left(\epsilon^{2} \sigma^{2}\right)\left(\bar{u}^{*} \bar{u}_{x^{*} x^{*}}-\bar{u}_{x^{*}}^{* 2}\right) \\
\beta^{*}=\left(\epsilon \sigma^{2}\right)\left(h \bar{u}^{*}\right)_{x^{*} t^{*}}+\left(\epsilon^{2} \sigma^{2}\right)\left[\bar{u}^{*}\left(h \bar{u}^{*}\right)_{x^{*} x^{*}}-\left(h \bar{u}^{*}\right)_{\left.x^{*} \bar{u}_{x^{*}}^{*}\right]}\right. \\
\gamma^{*}=\left(\epsilon \sigma^{2}\right)\left(h_{t}\right)_{t^{*}}^{*}+\left(\epsilon^{2} \sigma^{2}\right)\left[\bar{u}^{*}\left(h_{t}\right)_{x^{*}}^{*}-\left(h_{t}\right)^{*} \bar{u}_{x^{*}}^{*}\right]
\end{gathered}
$$

and substitution of (2.27) and (2.32) in equation (2.31) gives the following sc aleel momentum equation :

$$
\begin{array}{r}
(\epsilon \sigma) \frac{\partial \bar{u}^{*}}{\partial t^{*}}+\left(\epsilon^{2} \sigma\right) \bar{u}^{*} \frac{\partial \bar{u}^{*}}{\partial x^{*}}+(\epsilon \sigma) \frac{\partial \eta^{*}}{\partial x^{*}}=\sigma \frac{\left(h+\epsilon \eta^{*}\right)}{2} \frac{\partial}{\partial x^{*}}\left[\left(\epsilon \sigma^{2}\left(h_{\imath}\right)_{t^{*}}^{*}+O\left(\epsilon^{2} \sigma^{2}\right)\right]\right. \\
+\frac{\sigma}{H}\left(\epsilon^{3} \frac{\eta^{* 3}}{3}+\epsilon^{2} \frac{\eta^{* 2} h}{2}-\frac{h^{3}}{6}\right) \frac{\partial}{\partial x^{*}}\left[\left(\epsilon \sigma^{2}\right) \bar{u}_{x^{*} t^{*}}+O\left(\epsilon^{2} \sigma^{2}\right)\right] \\
+\epsilon \sigma \frac{\partial \eta^{*}}{\partial x^{*}}\left[\epsilon \eta^{*}\left(\epsilon \sigma^{2} \bar{u}_{x^{*} t^{*}}\right)+O\left(c^{2} \sigma^{2}\right)\right] \\
+\epsilon \sigma \frac{\partial \eta^{*}}{\partial x^{*}}\left[\left(\epsilon \sigma^{2}\right)\left(h_{t}\right)_{t^{*}}+\left(\epsilon \sigma^{2}\right)\left(h \bar{u}^{*}\right)_{x^{*} t^{*}}+O\left(\epsilon^{2} \sigma^{2}\right)\right]
\end{array}
$$

Keeping only the terms larger than $O\left(\epsilon^{2} \sigma^{3}\right)$, we obtain

$$
\begin{aligned}
(\epsilon \sigma) \frac{\partial \bar{u}^{*}}{\partial t^{*}}+\left(\epsilon^{2} \sigma\right) \bar{u}^{*} \frac{\partial \bar{u}^{*}}{\partial x^{*}}+(\epsilon \sigma) \frac{\partial \eta^{*}}{\partial x^{*}}= & \left(\epsilon \sigma^{3}\right) \frac{h}{2}\left[\left(h_{t}\right)^{*}+\left(h \bar{u}^{*}\right)_{x^{*}}\right]_{\iota^{*} s^{*}} \\
& -\left(\epsilon \sigma^{3}\right) \frac{h^{2}}{6} \bar{u}_{x^{*} t^{*} x^{*}}+()\left(c^{2} \sigma^{3}\right)
\end{aligned}
$$

in which it was assumed that $\frac{h^{3}}{H}=h^{2}$. Finally, we can go back to the original non-dimensional variables to get

$$
\bar{u}_{t}+\overline{u u}_{x}+\eta_{x}=\frac{h}{2}\left[h_{t}+(h \bar{u})_{x}\right]_{t x}-\frac{h^{2}}{6} \bar{u}_{x t x}+O\left(\epsilon^{2} \sigma^{3}\right)
$$




$$
\begin{aligned}
= & \frac{h^{2}}{3} \bar{u}_{x t x}+\frac{h}{2} h_{x x} \bar{u}_{t}+\frac{h}{2} h_{x}\left(\bar{u}_{x t}+\bar{u}_{t x}\right) \\
& +\frac{h}{2} h_{t t x}+\frac{h}{2}\left(h_{x t}+h_{t x}\right) \bar{u}_{x}+\frac{h}{2} h_{x t x} \bar{u} \\
& +\frac{h}{2} h_{t} \bar{u}_{x x}+O\left(\epsilon^{2} \sigma^{3}\right) .
\end{aligned}
$$

Lyuatuon (2.33) constitutes a 'low-order' representation of the 'complete' momentum cquation (224). In fact, (2.33) together with the continuity equation (2.9) represent the Boussinesq formulation of the equations governing waves generated by a moving lod. The assumptions made in the derivation of (2.33) imply that the waves of interest are nonlinear, dispersive, shallow-water waves. Equation (2.9) and (2.33) thenefore constitute a generalisation of the well-known Boussinesq system for waves over a flat bed.

It is important to note that, since $x$ and $t$ are both assumed to be of the same order, the operator $\frac{\partial^{2}}{\partial x \partial t}$ in equation $(2.33)$ can be replaced by $\frac{\partial^{2}}{\partial t \partial x}$ which gives the following equation :

$$
\begin{aligned}
\bar{u}_{t}+\overline{u u}_{x}+\eta_{x}= & \frac{h}{2}\left[h_{t}+(h \bar{u})_{x}\right]_{x t}-\frac{h^{2}}{6} \bar{u}_{x x t}+O\left(\epsilon^{2} \sigma^{3}\right) \\
= & \frac{h^{2}}{3} \bar{u}_{x x t}+\frac{h}{2} h_{x x} \bar{u}_{t}+h h_{x} \bar{u}_{x t} \\
& +\frac{h}{2} h_{t x t}+h h_{x t} \bar{u}_{x}+\frac{h}{2} h_{x x t} \bar{u} \\
& +\frac{h}{2} h_{t} \bar{u}_{x x}+O\left(\epsilon^{2} \sigma^{3}\right) .
\end{aligned}
$$

The order of appearance of the $x$ and $t$ partial derivatives only depends on the pocchlure used to obtain the final governing equation. In the derivation of (2.33), we assumed $u(x, y, t) \simeq \bar{u}(x, t)$ and depth-averaged the resulting $x$-momentum equation (2.13). All these terms of equation (2.24) (or equation 2.33) which would not appear in the standard Airy equation and which represent the deviation of the pressure from the hydrostatic state thus came from the depth-integration of the horizontal pressure gradient $\frac{\partial_{y}}{\partial_{3}}$.

However a different procedure can be used. We can start by stating that, to an order $O(t \sigma)$, the vertical velocity $v(x, y, t)$ is given exactly by the expression on the 
right hand side of approximation $(2.12)$, i.e.

$$
v(x, y, t)=-\frac{\partial h}{\partial t}-\frac{\partial}{\partial x}(h \bar{u})-y \frac{\partial \bar{u}}{\partial x}+O\left(\epsilon^{2} \sigma\right) .
$$

Then from the irrotationality condition (2.4) (which was not used in the derivation of (2.33)) we can deduce (Appendix A):

$$
u=\bar{u}-\left(y+\frac{h}{2}\right)\left[h_{t}+(h \bar{u})_{x}\right]_{x}-\left(\frac{y^{2}}{2}+\frac{h^{2}}{6}\right) \bar{u}_{2 x}+O\left(c^{2} \sigma^{2}\right) .
$$

Furthermore, the expression (2.21) for the pressure derived earlier can be writien an

$$
p=(\eta-y)+y\left[h_{t}+(h \bar{u})_{x}\right]_{t}+\frac{y^{2}}{2} \pi_{x t}+O\left(\iota^{2} \sigma^{2}\right) .
$$

Substitution of (2.35)-(2.37) in the $x$-momentum equation (2.2) gives

$$
\begin{aligned}
\bar{u}_{t}+\bar{u}_{x}+\eta_{x}= & \left(y+\frac{h}{2}\right)\left[h_{t}+(h \bar{u})_{x}\right]_{2 t}+\left(\frac{y^{2}}{2}+\frac{h^{2}}{6}\right) \bar{u}_{\lambda \perp t} \\
& -y\left[h_{t}+(h \bar{u})_{x}\right]_{t x}-\frac{y^{2}}{2} \bar{u}_{x t x}+O\left(\epsilon^{2} \sigma^{3}\right)
\end{aligned}
$$

which implies that $\frac{\partial^{2}}{\partial t \partial x}=\frac{\partial^{2}}{\partial x \partial t}$ and leads to equation (2.33) or (2.34). 'The above procedure is incorporated more systematically in a perturbation approach of the problem in which the basic variables are expanded in terms of the small parameters $\epsilon$ and $\sigma . \mathrm{Wu}(1981)$ used such a procedure to obtain an equation identical to (2.3.1) Appendix B shows a derivation of (2.34) using an expansion method.

Since the dispersive term usually appears as $\bar{u}_{x x t}$ in the Boussinesc systenll, we will henceforth used the formulation (2.34). It is worth noticing that, altomgh temm of $O\left(\epsilon^{2} \sigma^{3}\right)$ were left out from (2.34), the last term $\frac{h}{2} h_{t} \bar{u}_{x x}$ is also $O\left(\epsilon^{2} \sigma^{3}\right)$ accosdung tes the scales introduced at the beginning of this section. That contradiction onigmatres in the term $(h \bar{u})_{x x t}$ which is $O\left(\epsilon \sigma^{3}\right)$ but gives rise to $\frac{h}{2} h_{t} \bar{u}_{x x}$, a term of $O\left(c^{2} \sigma^{\cdot 3}\right)$, wher II the partial differentiation is carried out. Therefore the presence of a term of ()$\left(r^{2} \sigma^{3}\right)$ in the final equation is only due to a slight scaling inconsistency and for that rensen, the term $\frac{h}{2} h_{t} \bar{u}_{x x}$ is not eliminated from the final equation.

As it was done for the 'high-order' equation (2.24), we can reduce the govermmn equation for the cases of nonuniform and flat beds. If the bed is sloping but rost. 
moving, equation (2.34) reduces to

$$
\begin{aligned}
\bar{u}_{t}+\overline{u u}_{x}+\eta_{x} & =\frac{h}{2}\left[(h \bar{u})_{x}\right]_{x t}-\frac{h^{2}}{6} \bar{u}_{x x t}+O\left(\epsilon^{2} \sigma^{3}\right) \\
\cdot & =\frac{h^{2}}{3} \bar{u}_{x x t}+\frac{h}{2} h_{x x} \bar{u}_{t}+h h_{x} \bar{u}_{x t}+O\left(\epsilon^{2} \sigma^{3}\right)
\end{aligned}
$$

as denved by Peregrine (1967) in the study of long waves on beaches. If we also restrict the bed to a zero slope, we obtain the basic Boussinesq equation:

$$
\bar{u}_{t}+\overline{u u}_{x}+\eta_{x}=\frac{h^{2}}{3} \bar{u}_{x x t}+O\left(\epsilon^{2} \sigma^{3}\right)
$$

In the next section, equation (2.34) together with the continuity equation (2.9) will be used to model problems of waves generated by a moving bed. 


\section{NUMERICAL SOLUTIONS}

Because of the nonlinear and dispersive terms appearing in the governing equations obtained in $\$ 2.2$, it is extremely difficult (if not impossible) in general to derive analytical solutions to those equations. For that reason, we have to resont to a numerical method to obtain approximate solutions for the amplitude $\eta$ and velurilly

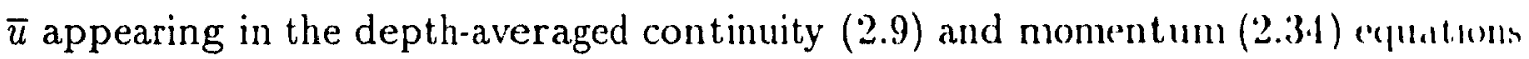

\subsection{The finite difference method}

The finite difference method is one of the oldest numerical methods uned III the solution of differential equations. Because of its simplicity and reliability, it still constitutes a very widespread tool for the numcrical solution of fluid flow problems For the sake of completeness, a brief elementary review of various aspects of the fintle difference method follows.

The basis of the finite difference method consists in replacing a function dedimed over a continuous solution domain by approximations of that function evaluated at discrete locations of the solution domain called grid points. These approxinntions may then be used to express the derivatives of the function in tcrms of algelliar equations called finite difference equations. The important conseguence of the fimt. difference method is therefore the possibility to transform one or more diffrimlintul equations into an equivalent algebraic problem.

In the present problem, we are concerned with the approximation of dependent flow variables such as $\eta, \bar{u}$ and $h$ which are functions of spatial position $x$ and time $t$. Thus, we may consider an arbitrary function $f(x, t)$ which is assumed to b. the solution of a boundary value problem defined over a domain $0 \leq x \leq x_{N}$ and $0 \leq 1 \leq$ $t_{P}$. The starting point of the finite difference method is to replace this contimum solution domain by a discrete set of points denoted by $\left(x_{1}, t_{j}\right)$ such that $0 \leq x_{1} \leq s_{N}$ and $0 \leq t_{j} \leq t_{P}$ where $i=0,1, \ldots, N$ and $j=0,1, \ldots, P$ (Figure 3.1). The solutum domain thus takes the appearance of a grid and for that reason the points $\left(x_{1}, t,\right)$ are 
called grid points. The mesh size in the $t$-direction is constant and denoted by $\Delta t$ so that $t_{P}=P \Delta t$. The spatial mesh size is denoted by $\Delta x_{i}$, a distance which may vary with time. The finite difference approximation of $f(x, t)$ evaluated at the grid point $(\imath, \jmath)$ is denoted by $f_{1}^{?}$.

\subsubsection{Eulerian description}

Most of the finite difference models used in fluid mechanics and hydraulics involve an Eulerian description of the flow field. That implies that the flow variables are calculated at fixed locations in the solution domain and therefore that the grid points spacing in the $x$-direction does not change with time. We will only consider the case where the spatial mesh size is constant, i. e. $\Delta x_{1}=\Delta x$ such that $x_{N}=N \Delta x$ (Figure $3.1)$

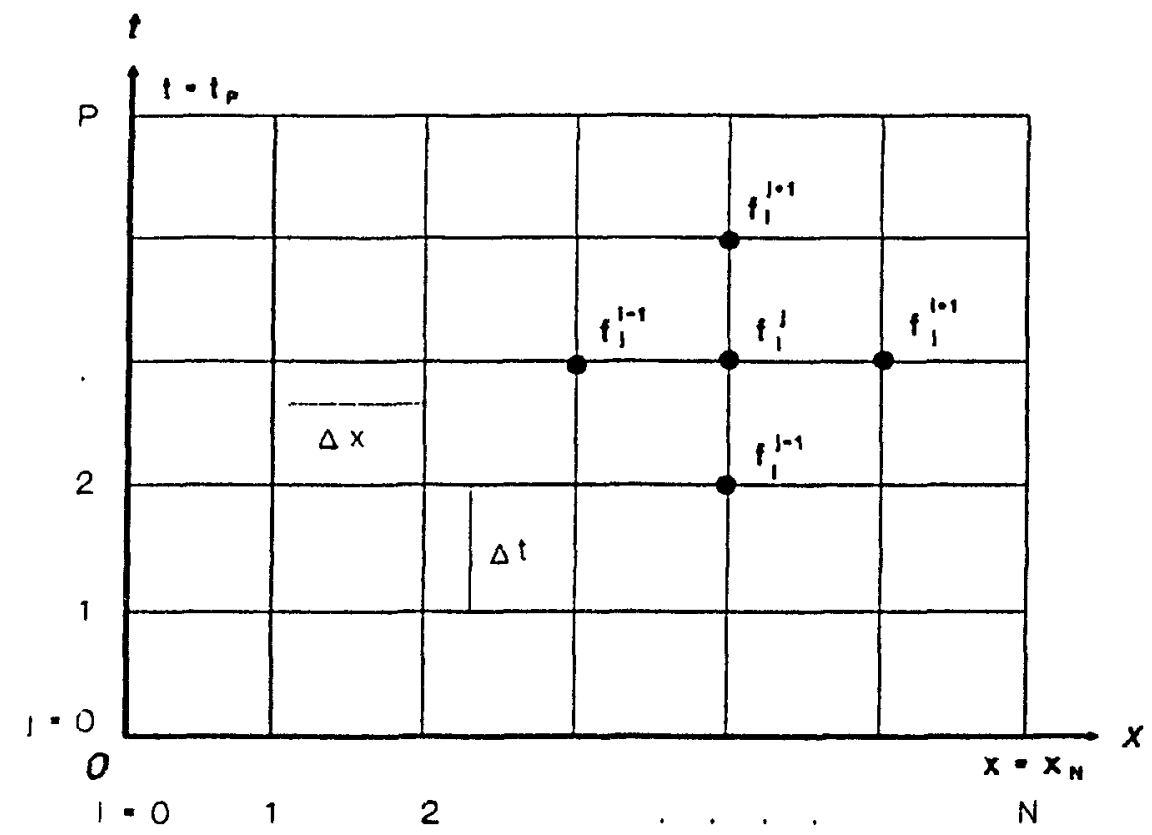

Figure 3.1: The Eulerian finite difference problem.

At any given time, if $f(x, t)$ has continuous spatial derivatives, it can be approximated in the neighborhood of $x=x_{1}$ by the following Taylor series:

$$
f(x, t)=f_{i}+\left(x-x_{1}\right) \frac{\partial f}{\partial x_{i}}+\frac{\left(x-x_{i}\right)^{2}}{2} \frac{\partial^{2} f}{\partial x_{\imath}^{2}}+\frac{\left(x-x_{i}\right)^{3}}{3 !} \frac{\partial^{3} f}{\partial x_{i}^{3}}+\cdots
$$


where $f_{t}$ denotes the finite difference approximation of $f\left(x_{1}, t\right)$. Using this Thylon series, we can write the following approximation for $f\left(x_{1+1}, t\right)$ :

$$
f_{\imath+1}=f_{1}+\Delta x \frac{\partial f}{\partial x_{\imath}}+\frac{\Delta x^{2}}{2} \frac{\partial^{2} f}{\partial x_{1}^{2}}+\frac{\Delta x^{3}}{3 !} \frac{\partial^{3} f}{\partial x_{1}^{3}}+O\left(\Delta x^{4}\right)
$$

The series (3.1) directly gives a finite difference approximation for $\frac{\partial f}{\partial x}$ evalualed at $\dot{x}=x_{\imath}$ :

$$
\frac{\partial f}{\partial x_{1}}=\frac{f_{1+1}-f_{1}}{\Delta x}+O(\Delta x)
$$

In a similar way, we can approximate $f\left(x_{t-1}, t\right)$ by the series

$$
f_{\imath-1}=f_{\imath}-\Delta x \frac{\partial f}{\partial x_{2}}+\frac{\Delta x^{2}}{2} \frac{\partial^{2} f}{\partial x_{\imath}^{2}}-\frac{\Delta x^{3}}{3 !} \frac{\partial^{3} f}{\partial x_{\mathfrak{\imath}}^{3}}+O\left(\Delta x^{4}\right)
$$

from which we obtain

$$
\frac{\partial f}{\partial x_{i}}=\frac{f_{i}-f_{i-1}}{\Delta x}+O(\Delta x)
$$

Equations (3.2) and (3.4) are respectively called forward and backward differme. equations. In both cases the approximation involves an error tem of $O(\Delta . r)$. 'The' magnitude of this error term is reduced if we subtract (3.3) from (3.1) to obtann

$$
\frac{\partial f}{\partial x_{i}}=\frac{f_{i+1}-f_{i-1}}{2 \Delta x}+O\left(\Delta x^{2}\right)
$$

which is a central difference representation of $\frac{\partial f}{\partial x}$. A finite difference approximation for $\frac{\partial^{2} f}{\partial x^{2}}$ at $x=x_{1}$ can be obtained by addition of (3.1) and (3.3) :

$$
\frac{\partial^{2} f}{\partial x_{1}^{2}}=\frac{f_{\imath+1}-2 f_{\imath}+f_{1-1}}{\Delta x^{2}}+O\left(\Delta x^{2}\right)
$$

We follow a similar procedure to derive finite difference approximations for the time derivatives of $f(x, t)$. At any given location in space, approximations for $f\left(x, t_{3+1}\right)$ and $f\left(x, t_{\jmath}\right)$ in the neighborhood of $t=t_{\jmath+\frac{1}{2}}$ can be written as

$$
f^{\jmath+1}=f^{\jmath+\frac{1}{2}}+(\Delta t / 2) \frac{\partial f}{\partial t_{\jmath+\frac{1}{2}}}+\frac{(\Delta t / 2)^{2}}{2} \frac{\partial^{2} f}{\partial t^{2}{ }^{2}+\frac{1}{2}}+O\left(\Delta t^{3}\right)
$$

and

$$
f^{\jmath}=f^{\jmath+\frac{1}{2}}-(\Delta t / 2) \frac{\partial f}{\partial t_{j+\frac{1}{2}}}+\frac{(\Delta t / 2)^{2}}{2} \frac{\partial^{2} f}{\partial t_{j+\frac{1}{2}}^{2}}+O\left(\Delta t^{3}\right)
$$


Subtraction of (3.8) from (3 7) gives an approximation of $\frac{\partial f}{\partial t}$ evaluated at $t=t_{\jmath+\frac{1}{2}}$ :

$$
\frac{\partial f}{\partial t_{\jmath+\frac{1}{2}}}=\frac{f^{\jmath+1}-f^{\jmath}}{\Delta t}+O\left(\Delta t^{2}\right)
$$

and addition of the two series gives :

$$
\frac{\partial^{2} f}{\partial t_{j+\frac{1}{2}}^{2}}=\frac{f^{\jmath+1}-2 f^{\jmath+\frac{1}{2}}+f^{\jmath}}{(\Delta t / 2)^{2}}+O\left(\Delta t^{2}\right)
$$

\subsubsection{Lagrangian description}

In the Lagrangian description of the flow field, the flow variables are not evaluated in a fixed reference frame but rather at the instantaneous locations of the moving flund particles. In the finite difference method, that implies that the grid moves with the fluid partıcles and therefore that the spatial mesh size varies with time, 1 e. $\Delta x_{1}=\Delta x_{1}(t)=\frac{1}{2}\left(x_{t+1}-x_{i-1}\right)$ for $i=1,2, \ldots, N-1$. Depending on the motion of the fluid, the space-time grid can then take the distorted appearance of figure 3.2 .

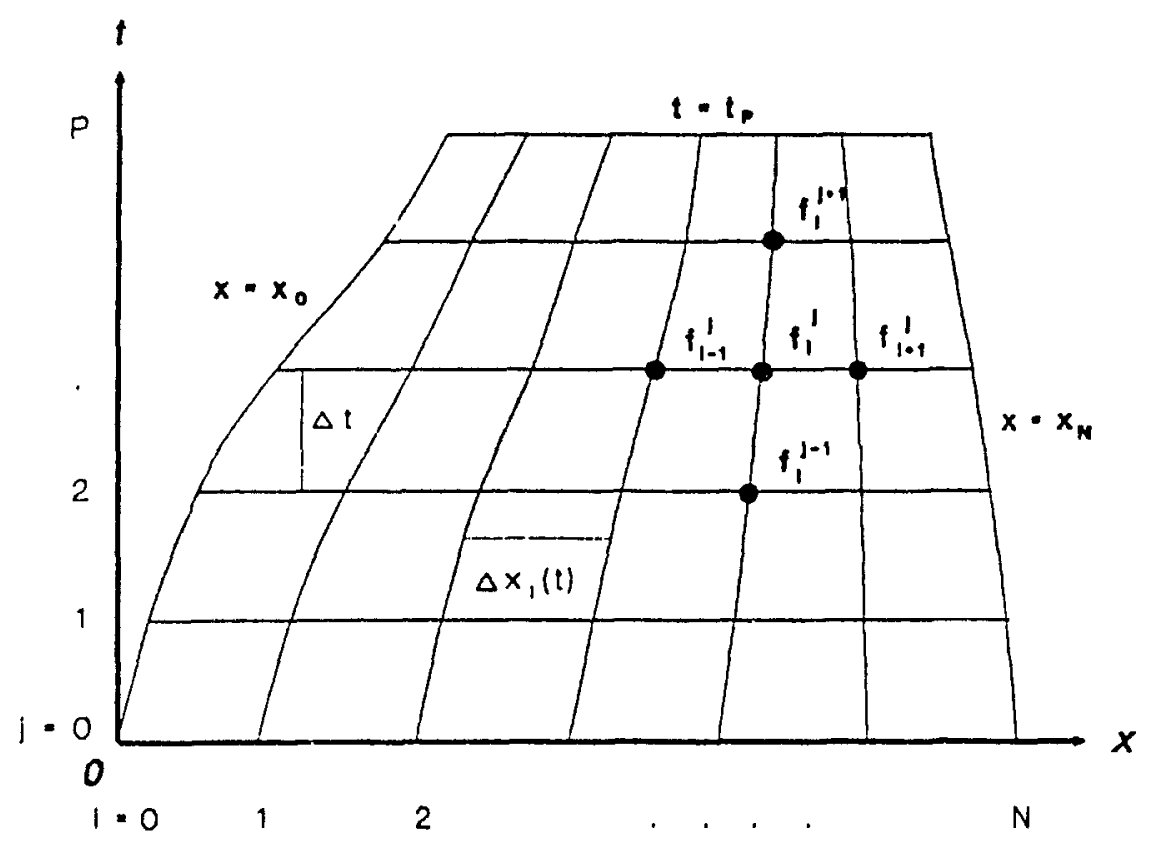

Figure 3.2: The Lagrangian finite difference problem. 
If at in given time we let $\Delta x=x_{t+1}-x_{1}$ and $\Delta x^{\prime}=x_{1}-x_{1-1}$ (Figure 3.3), we can approximate $f\left(x_{0+1}, t\right)$ and $f\left(x_{1-1}, t\right)$ by power series about $x=x_{1}$ :

$$
\begin{aligned}
& f_{i+1}=f_{i}+\Delta x \frac{\partial f}{\partial x_{i}}+\frac{\Delta x^{2}}{2} \frac{\partial^{2} f}{\partial x_{1}^{2}}+O\left(\Delta x^{3}\right) \\
& f_{i-1}=f_{i}+\Delta x^{\prime} \frac{\partial f}{\partial x_{i}}+\frac{\Delta x^{\prime 2}}{2} \frac{\partial^{2} f}{\partial x_{i}^{2}}+O\left(\Delta x^{3}\right)
\end{aligned}
$$

Subtraction of (3.12) from (3.11) gives

$$
\begin{aligned}
f_{\imath+1}-f_{\imath-1} & =\left(\Delta x+\Delta x^{\prime}\right) \frac{\partial f}{\partial x_{\imath}}+\frac{1}{2}\left(\Delta x^{2}-\Delta x^{\prime 2}\right) \frac{\partial^{2} f}{\partial x_{i}^{2}}+O\left[\max \left(\Delta x^{3}, \Delta x^{\prime \prime \prime}\right)\right] \\
& =2 \Delta x_{i} \frac{\partial f}{\partial x_{i}}+\frac{1}{2}(2 \delta x)\left(2 \Delta x_{\imath}\right) \frac{\partial^{2} f}{\partial x_{i}^{2}}+O\left[\max \left(\Delta x^{3}, \Delta x^{\prime 3}\right)\right]
\end{aligned}
$$

where $\delta x=\frac{1}{2}\left(\Delta x-\Delta x^{\prime}\right)$ (Figure 3.3). The central difference formula for the tin it

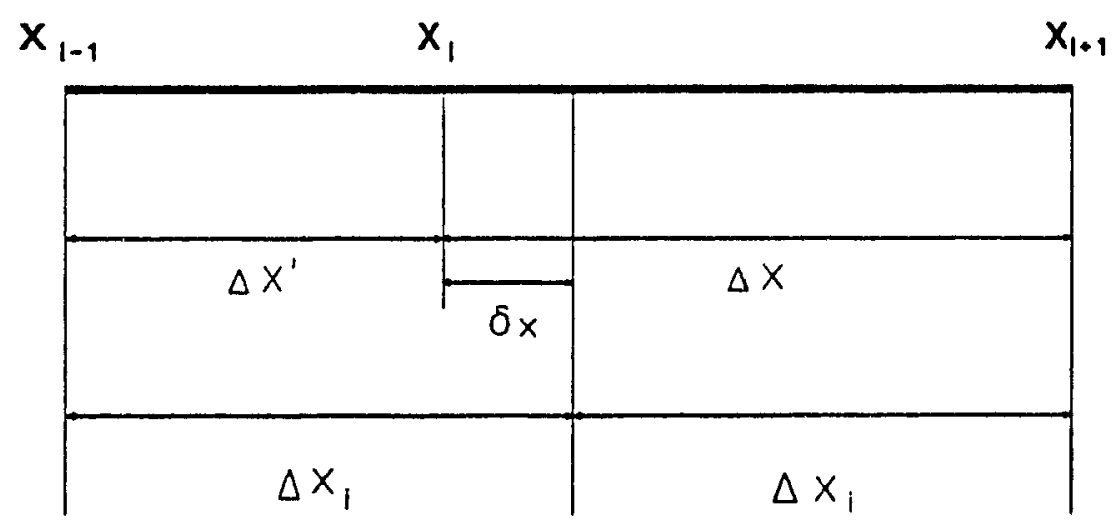

Figure 3.3: The Lagrangian grid.

order spatial derivative at $x=x_{1}$ is then

$$
\frac{\partial f}{\partial x_{i}}=\frac{f_{i+1}-f_{i-1}}{2 \Delta x_{i}}+O\left[\max \left(\delta x, \Delta x^{2}, \Delta x^{\prime 2}\right)\right]
$$

The error term in (3.13) is a function of the grid deformation. For a fixed grid of uniform mesh size, $\delta x=0, \Delta x^{\prime}=\Delta x$ and (3.13) is identical to (3.5). However as the distortion of the grid increases, the approximation (3.13) increasingly looses accuracy (Boris 1981). Addition of (3.11) and (3.12) gives

$$
\frac{\partial^{2} f}{\partial x_{1}^{2}}=\frac{f_{i+1}-2 f_{2}+f_{i-1}}{\frac{1}{2}\left(\Delta x^{2}+\Delta x^{\prime 2}\right)}+\cdots
$$


which is also subject to an error term depending on the grid deformation.

For the time derivatives, a procedure similar to the one used in the Eulerian case is followed. The important difference is that the resulting derivatives are not evaluated locally but rather following the fluid. They are total (or material) derivatives and are expressed as :

$$
\frac{d f_{i}}{d t_{\jmath+\frac{1}{2}}}=\frac{f_{i}^{\jmath+1}-f_{i}^{3}}{\Delta t}+O\left(\Delta t^{2}\right)
$$

ind

$$
\frac{d^{2} f_{1}}{d t_{j+\frac{1}{2}}}=\frac{f_{i}^{j+1}-2 f_{i}^{j+\frac{1}{2}}+f_{i}^{\jmath}}{(\Delta t / 2)}+O\left(\Delta t^{2}\right)
$$

\subsubsection{Explicit and implicit formulations}

When the finite difference method is applied to time-dependent problems, the resultIng algebraic equations may etther have an explicit or an implicit solution. Therefore a finite difference scheme can be classified as explicit or implicit according to the algebraic problem to which it leads.

The explicit form may be represented schematically by figure 3.4 in which the dots and crosses indicate grid points needed in the computations of spatial and temporal derivatives respectively. In that type of scheme, the calculation of the solution at the 'new' tıme is only based on the solution at the previous time.

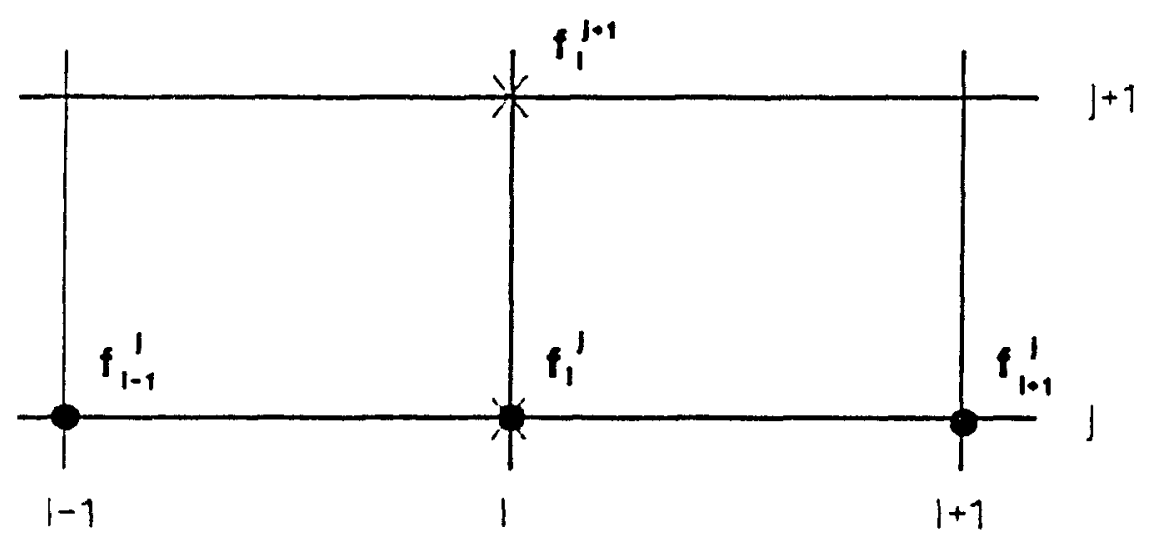

Figure 3.4: The explicit form. 
In an implicit scheme, the unknown solution at the new time not only depends on the solution at the previous time but also on values of the function at this new tume This type of scheme leads to a system of equations which must be solved smmultanc'ously. Figure 3.5 represents a fully implicit scheme in which all the derlvatives ate

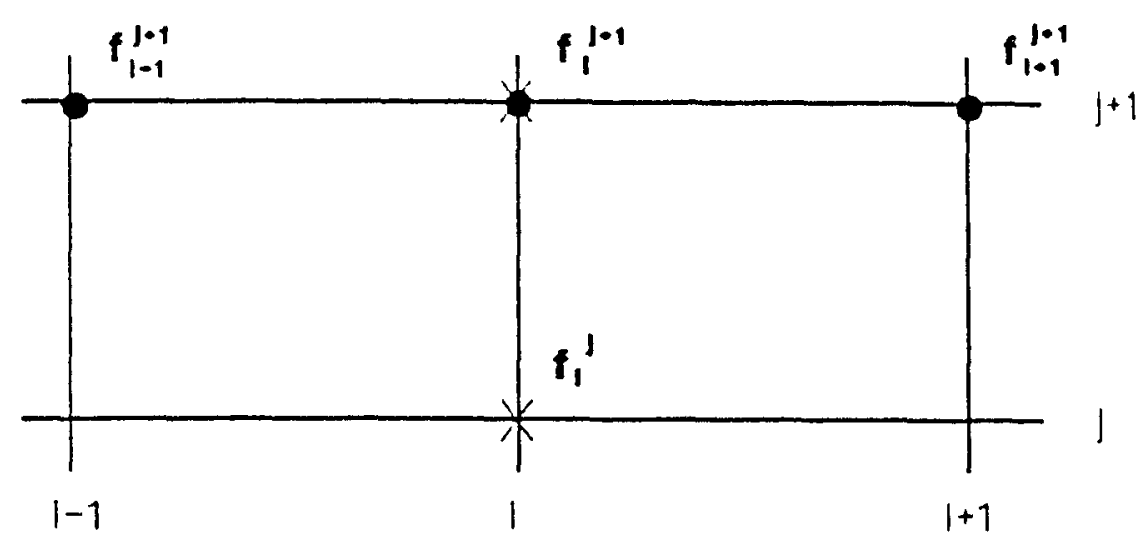

Figure 3.5: The fully implicit form.

evaluated at the advanced time. Figure 3.6 shows an implicst scheme: proporen by Crank and Nicolson in 1947. The derivatives are now evaluated at the intermenliat." time and calculated by averaging values at the previous and advanced times.

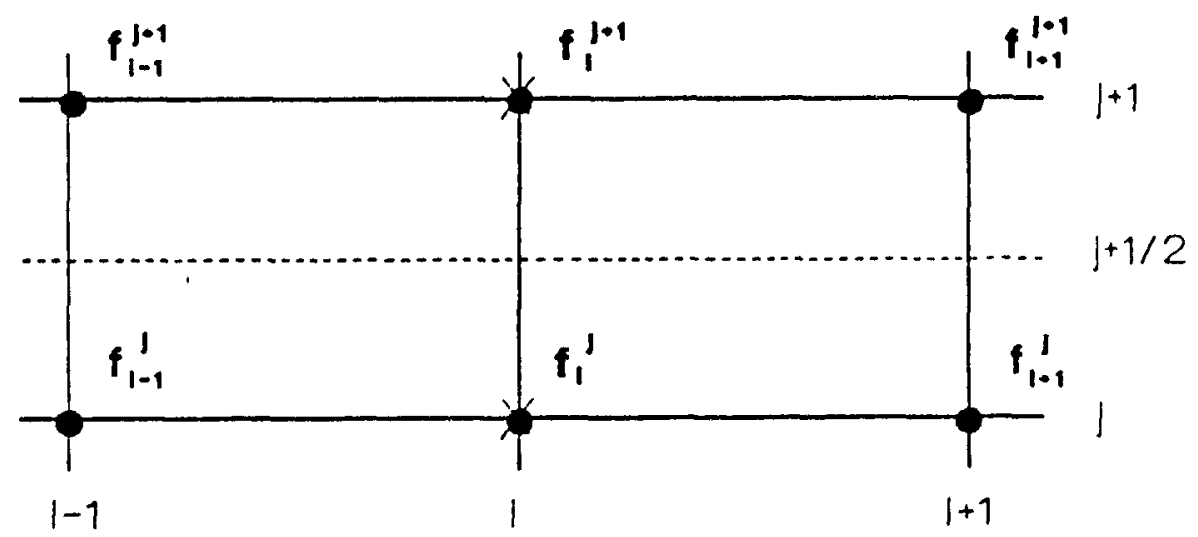

Figure 3.6: The Crank-Nicolson form.

Explicit schemes have the advantage of being simple to formulate but they are subject to stability conditions which impose certain limitations on the mesh size. (On 
the other hand, implicit schemes can be shown to be unconditionally stable but their formulation is often complex and requires longer computation times.

In the next section the equations derived in $\S 2.2$ will be formulated in terms of explicit and implicit finite difference methods.

\subsection{Finite difference formulation of the governing equations}

We will now use the basic principles developed in $§ 3.1$ to obtain finite difference formulations for the governing continuity and momentum equations.

\subsubsection{Choice of the flow field description}

The first question which must be answered in the application of the finite difference mothod to the governing equations (2.9) and (2.34) is whether to use the Eulerian or Lagrangian specification of the flow field.

Equations (2.9) and (2.34) were derived from the fundamental Eulerian equations of motion (2.1 to 2.3). It would therefore appear natural to use the Eulerian finite difference method introduced in §3.1.1. As already pointed out, that method implies that the spatial grid points are not associated with the moving fluid particles (Lagrangian specification) but rather with evenly spaced fixed locations in the flow field.

However, in some moving bed problems, the water surface may intersect the moving segment of the bed at a point of zero depth. In such problems, the upstream boundary of the flow field is constituted by a moving waterline. The 'moving wedge' problem mentioned in $\S 1$ and presented in $\S 4.1$ is an example of such a case. The major disadvantage implied in the Eulerian treatment of these problems is that the number of grid points involved in the description of the free surface may constantly vary. That cocates difficulties in the computer coding of the scheme and almost mariably requires interpolation or extrapolation procedures. The above remarks could also be made about the Eulerian treatment of wave run-up on beaches in which 
various methods must be used to overcome the difficulties associated with the shoreline boundary (see for example Hibberd \& Peregrine 1979).

The obvious alternative in problems involving a moving waterline is to use a Lagrangian description of the flow field. In that way, the location of that point of zero depth is easily followed since it always corresponds to one of the moving grid points.

We will now describe both the Eulerian and the Lagrangan finte difference for mulations of the governing equations (2.9) and (2.34).

\subsubsection{Eulerian scheme}

The functions $\eta, \bar{u}$ and $h$ are all specified on the same spatial grid points. Figure $3 i$ shows the arrangment of the flow field at any given time. Since we are only dealing with equations in one spatial dimension, the grid points actually correspond to vertical interfaces separating fluid elements bounded by the bed and the free surface

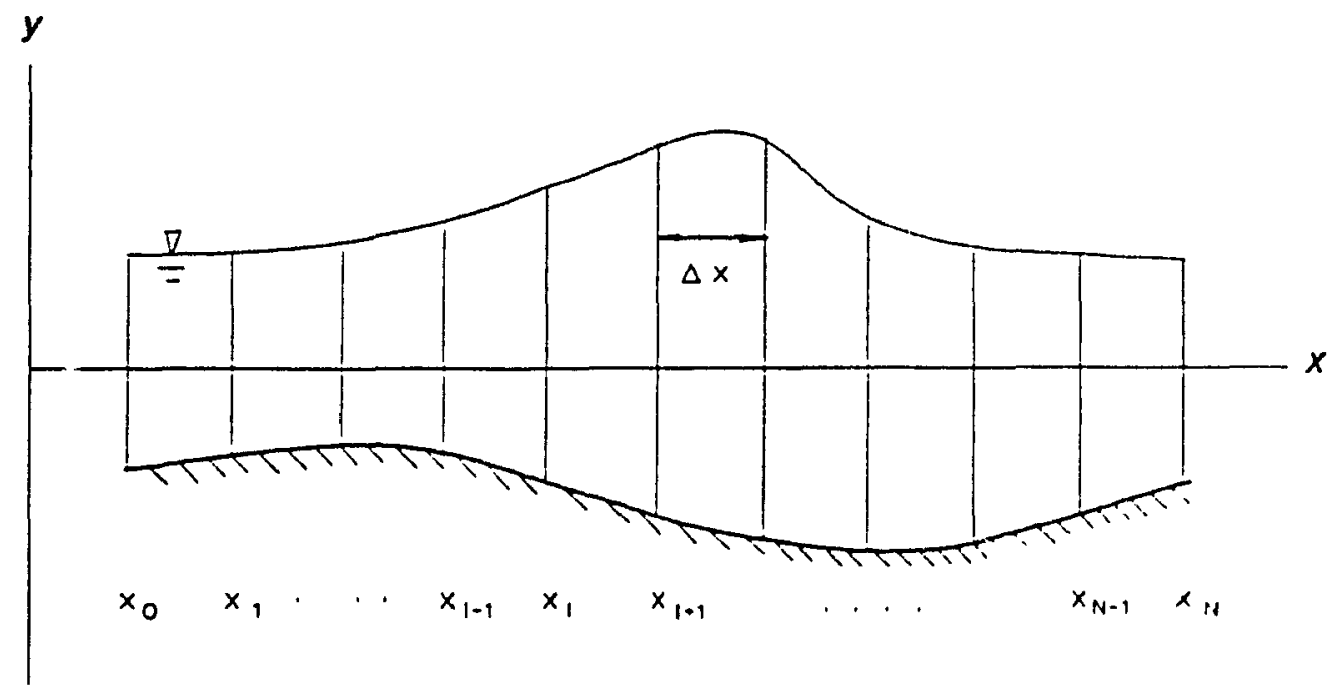

Figure 3.7: Eulerian description of the flow field.

The proposed numerical scheme is divided in three main parts :

1. Explicit solution of the depth-averaged continuity equation to obtain the 
predicted free surface position.

2. Implicit (Crank-Nicolson) solution of the momentum equation $(\dot{2} .34)$ to obtain the depth-averaged velocity.

3. Explicit solution of the depth-averaged continuity equation to obtain the corrected free surface position.

As a prerequisite to the application of the above scheme, we assume that initial conditions at $t=0$ and boundary conditions at $x=x_{0}$ and $x=x_{N}$ are known for the free surface position $\eta$ and the depth-averaged fluid velocity $\bar{u}$. Furthermore, we assume that the bed variation $h(x, t)$ is known everywhere in the solution domain.

We now look more closely into each of the above 3 steps :

- Explicul calculation of the predicted free surface position $\tilde{\eta}_{3}^{j+1}$.

The depth-averaged contınuity equation (2.9) can be written as

$$
\frac{\partial \eta}{\partial t}=-(h+\eta) \frac{\partial \bar{u}}{\partial x}-\bar{u} \frac{\partial \eta}{\partial x}-\bar{u} \frac{\partial h}{\partial x}-\frac{\partial h}{\partial t}
$$

Using the finite-difference formulae developed in $§ 3.1 .1$, we approximate (3.17) as :

$$
\begin{aligned}
& \tilde{\eta}_{1}^{j+1}=\eta_{1}^{j}-\Delta t\left[\frac{1}{2}\left(h_{\imath}^{j+1}+h_{\imath}^{J}\right)+\eta_{1}^{j}\right] \frac{\bar{u}_{i+1}^{J}-\bar{u}_{\imath-1}^{J}}{2 \Delta x} \\
& -\Delta t \bar{u}_{i}^{j}\left(\frac{\eta_{i+1}^{j}-\eta_{i-1}^{j}}{2 \Delta x}\right) \\
& -\Delta t \bar{u}_{i}^{\jmath}\left(\frac{h_{i+1}^{j+1}-h_{i-1}^{3+1}+h_{i+1}^{3}-h_{i-1}^{j}}{4 \Delta x}\right) \\
& -\left(h_{\mathrm{t}}^{3+1}-h_{\mathrm{i}}^{3}\right) \text {. }
\end{aligned}
$$

For known values of $\eta$ and $\bar{u}$ at time $t=t_{3}$, equation (3.18) can be used to calculate explicitly predicted values of the free surface position at time $t=t_{j+1}$ denoted by $i_{1}^{j+1}$ where $\imath=1,2, \ldots, N-1$. 
- Implicit calculatıon of the depth-averaged velocity $\bar{u}_{*}^{3+1}$.

With the help of the finite-difference formulae introduced in $\$ 3.1 .1$ and the ('ankNicolson scheme described in $\S 3.1 .3$, the depth-averaged momentum equation (2.3.1) can be written as :

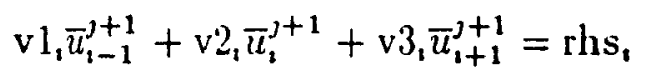

where

$$
\begin{aligned}
& \mathrm{vl}_{\mathrm{v}}=-\frac{\bar{u}_{\mathrm{s}}^{\jmath}}{4 \Delta x}-A+B+\frac{h_{\mathrm{t}}^{j+1}}{4 \Delta x} C-\frac{h_{\mathrm{t}}^{\jmath+1}}{2 \Delta x^{2}} \bar{u}_{\mathrm{\imath}}^{\jmath} m_{\mathrm{t}}^{\jmath+1}-\frac{h_{\mathrm{t}}^{\jmath+1}}{4 \Delta x^{2}} F \\
& \mathrm{v}_{2}=\frac{1}{\Delta t}+2 A-\frac{D}{\Delta t}-\frac{h_{\mathrm{z}}^{j+1}}{4} E+\frac{h_{1}^{j+1}}{\Delta x^{2}} \bar{u}_{\mathrm{a}}^{J} m_{\mathrm{a}}^{j+1}+\frac{h_{\mathrm{s}}^{j+1}}{2 \Delta x^{2}} F^{\prime} \\
& \mathrm{v} 3_{i}=\frac{\bar{u}_{i}^{J}}{4 \Delta x}-A-B-\frac{h_{i}^{j+1}}{4 \Delta x} C-\frac{h_{t}^{j+1}}{2 \Delta x^{2}} \bar{u}_{\imath}^{\jmath} m_{\imath}^{j+1}-\frac{h_{i}^{j+1}}{4 \Delta x^{2}} F
\end{aligned}
$$

and

$$
\begin{aligned}
& \mathrm{rhs}_{\imath}=\quad \vec{u}_{\mathfrak{\imath}-1}^{J}\left(\frac{\vec{u}_{\mathrm{i}}^{J}}{4 \Delta x}-A+B-\frac{h_{\mathrm{i}}^{\jmath}}{4 \Delta x} C+\frac{h_{1}^{\jmath}}{2 \Delta x^{2}} \bar{u}_{\mathrm{i}}^{\jmath} m_{\mathrm{i}}^{\jmath}+\frac{h_{\mathrm{i}}^{\jmath}}{4 \Delta x^{2}} F\right) \\
& +\bar{u}_{1}^{3}\left(\frac{1}{\Delta t}+2 A-\frac{D}{\Delta t}-\frac{h_{t}^{J}}{4} E-\frac{h_{1}^{J}}{\Delta x^{2}} \pi_{1}^{J} m_{1}^{J}-\frac{h_{2}^{J}}{2 \Delta x^{2}} l^{\prime}\right)
\end{aligned}
$$

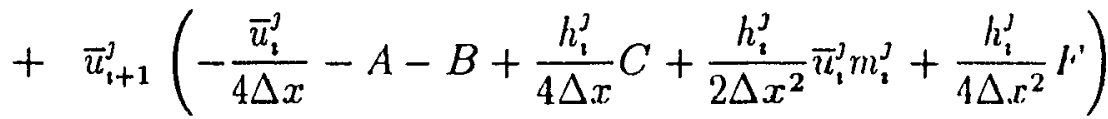

$$
\begin{aligned}
& -F+\left[\left(h_{\mathfrak{\imath}}^{j+1}+h_{\mathfrak{\imath}}^{j}\right)\left(r_{\mathfrak{1}+1}^{\jmath}-r_{\mathfrak{\imath}-1}^{\jmath}\right)\right] /\left(8 \Delta x^{2}\right)
\end{aligned}
$$

Also

$$
\begin{aligned}
& \left.A=\frac{1}{6}\left[\left(h_{\imath}^{\jmath}\right)^{2}+\left(h_{\imath}^{j+1}\right)^{2}\right)\right] \\
& B=\frac{1}{4 \Delta x}\left[h_{\mathfrak{\imath}}^{J}\left(h_{\mathfrak{\imath}+1}^{\jmath}-h_{\mathfrak{\imath}-1}^{\jmath}\right)+h_{\mathfrak{\imath}}^{j+1}\left(h_{\mathfrak{\imath}+1}^{j+1}-h_{\mathfrak{\imath}-1}^{j+1}\right)\right] \\
& C=\frac{1}{2 \Delta x \Delta t}\left(h_{t+1}^{j+1}-h_{\imath-1}^{j+1}-h_{\imath+1}^{3}+h_{\imath-1}^{j}\right) \\
& D=\frac{1}{4 \Delta x^{2}}\left[h_{\mathrm{i}}^{j}\left(h_{\mathrm{i}+1}^{j}-2 h_{\mathrm{\imath}}^{j}+h_{\imath-1}^{j}\right)+h_{\mathrm{i}}^{j+1}\left(h_{\mathrm{i}+1}^{j+1}-2 h_{\mathrm{i}}^{j+1}+h_{\mathrm{i}-1}^{j+1}\right)\right] \\
& E=\frac{1}{\Delta x^{2} \Delta t}\left[\left(h_{\mathfrak{t}+1}^{j+1}-2 h_{\mathrm{i}}^{j+1}+h_{\mathfrak{i}-1}^{j+1}\right)-\left(h_{\mathrm{t}+1}^{j}-2 h_{\mathrm{t}}^{3}+h_{\mathrm{t}-1}^{\jmath}\right)\right] \\
& F=\frac{1}{4 \Delta x}\left(\eta_{2+1}^{3+1}-\eta_{z-1}^{3+1}+\eta_{i+1}^{3}-\eta_{i-1}^{3}\right) \\
& m_{\imath}^{\jmath}=\frac{1}{2 \Delta x}\left(h_{\imath+1}^{\jmath}-h_{\imath-1}^{\jmath}\right) \\
& r_{1}^{\jmath}=\frac{1}{(\Delta t / 2)^{2}}\left(h_{\mathfrak{\imath}}^{3+1}-2 h_{\imath}^{3+\frac{1}{2}}+h_{\mathfrak{\imath}}^{3}\right)
\end{aligned}
$$


The only unknown in (3.19) is the velocity field $\bar{u}_{i}^{3+1}$ for $i=1,2, \ldots, N-1$ which olviously cannot be calculated explicitly. By rewriting (3.19) in matrix form as

$$
V \bar{l}^{\jmath+1}=\mathrm{RHS}
$$

(1)

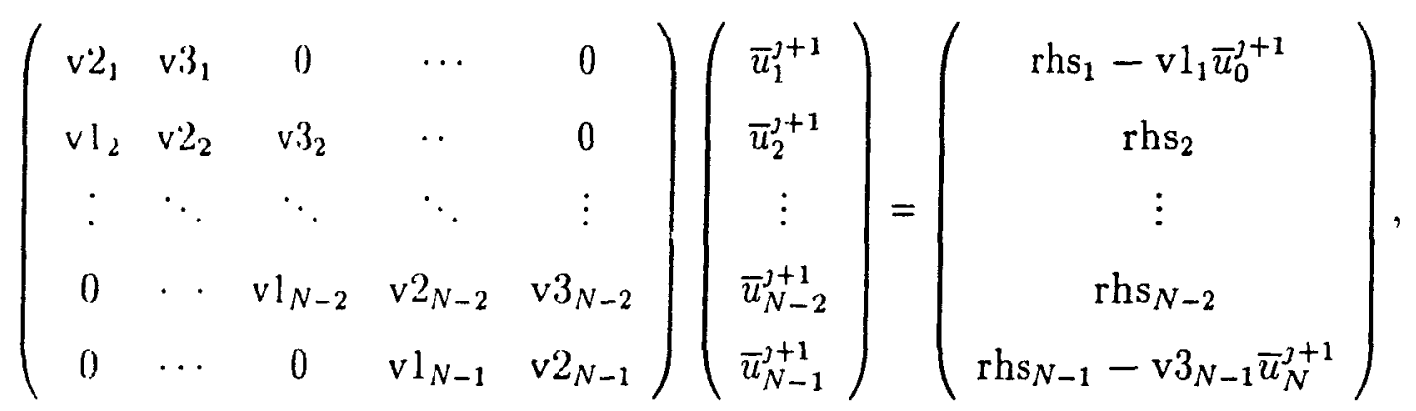

we see that this velocity field is the solution of a system of linear non-homogeneous rquations having a tridiagonal coefficient matrix. That type of system can be solved by a straightforward elimination procedure.

- Explicul calculation of the corrccted free surface position $\eta_{3}^{j+1}$.

In this last stcp, we calculate a corrected value $\eta_{1}^{j+1}$ for the free surface position by using the velocity field $\bar{u}_{1}^{3+1}$ calculated in the second step. Equation (2.9) is solved ixplicitly as.

$$
\begin{aligned}
& \eta_{1}^{j+1}=\eta_{1}^{j}-\Delta t\left[\frac{1}{2}\left(h_{\imath}^{\jmath+1}+h_{\imath}^{j}\right)+\eta_{\imath}^{\jmath}\right] \frac{\bar{u}_{\imath+1}^{j+1}-\bar{u}_{i-1}^{j+1}+\bar{u}_{\imath+1}^{J}-\bar{u}_{i-1}^{\jmath}}{4 \Delta x} \\
& -\Delta t\left(\frac{\bar{u}_{i}^{j+1}+\bar{u}_{i}^{\jmath}}{2}\right) \frac{\eta_{z+1}^{\jmath}-\eta_{z-1}^{\jmath}}{2 \Delta x} \\
& -\Delta t\left(\frac{\bar{u}_{t}^{j+1}+\bar{u}_{\imath}^{\jmath}}{2}\right) \frac{h_{\imath+1}^{j+1}-h_{t-1}^{j+1}+h_{t+1}^{\jmath}-h_{\imath-1}^{\jmath}}{4 \Delta x} \\
& -\left(h_{1}^{j+1}-h_{\imath}^{j}\right) \text {. }
\end{aligned}
$$

\subsubsection{Lagrangian scheme}

Figure (3.7) shows a Lagrangian configuration of the flow field. The important difDencuce with respect to the Eulerian representation of Figure (3.6) is that the fluid Interfaces on which $\eta, \bar{u}$ and $h$ are specified are now allowed to move. 


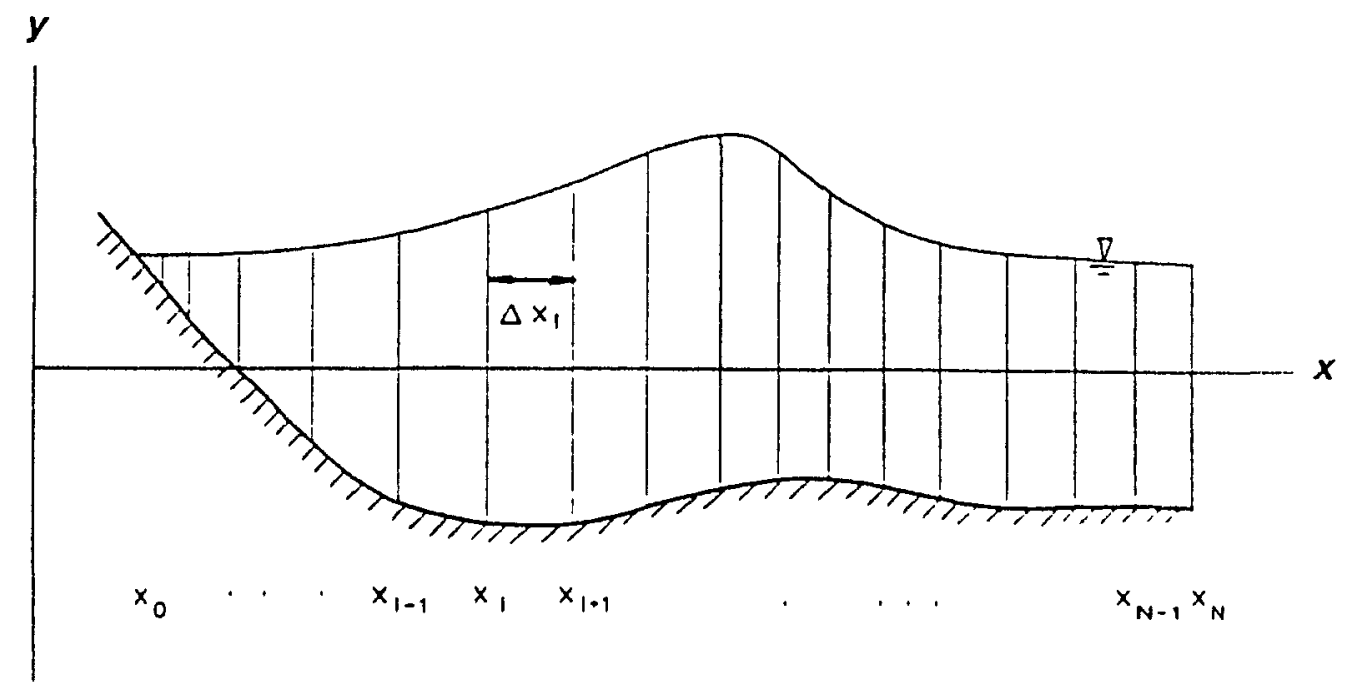

Figure 3.8: Lagrangian description of the flow field.

As mentioned before, the depth-averaged continuity and momentum equations (2.9) and (2.34) constitute Eulerian formulations. By introducing the material derivative (or 'derivative following the fluid')

$$
\frac{d}{d t}=\frac{\partial}{\partial t}+\bar{u} \frac{\partial}{\partial x}
$$

and applying it to the free surface position $\eta$

$$
\frac{d \eta}{d t}=\frac{\partial \eta}{\partial t}+\bar{u} \frac{\partial \eta}{\partial x}
$$

and to the depth-averaged velocity $\bar{u}$

$$
\frac{d \bar{u}}{d t}=\frac{\partial \bar{u}}{\partial t}+\bar{u} \frac{\partial \bar{u}}{\partial x},
$$

we can rewrite (2.9) and (2.34) as :

$$
\begin{gathered}
\frac{d \eta}{d t}=-(h+\eta) \bar{u}_{x}-\frac{d h}{d t} \\
\frac{d \bar{u}}{d t}=-\eta_{x}+\frac{h^{2}}{3} \frac{d \bar{u}_{x x}}{d t} \\
+h h_{x} \frac{d \bar{u}_{x}}{d t}+\frac{h}{2} \frac{d^{2} h_{x}}{d t^{2}}+h \bar{u}_{x} \frac{d h_{x}}{d t}+\frac{h}{2} \bar{u}_{x x} \frac{d h}{d t}
\end{gathered}
$$


Equation (3.22) is exact while (3.23) is valıd up to order $O\left(\epsilon^{2} \sigma^{3}\right)$. For waves over a flat bed, equation (3.22) and (3.23) reduce to :

$$
\begin{gathered}
\frac{d \eta}{d t}=-(h+\eta) \bar{u}_{x} \\
\frac{d \bar{u}}{d t}=-\eta_{x}+\frac{h^{2}}{3} \frac{d \bar{u}_{x x}}{d t}
\end{gathered}
$$

Taking the material derivative of $x$, we also obtain

$$
\frac{d x}{d t}=\bar{u}
$$

which can be used to define the position interfaces $x_{z}(i=0,1, \ldots, N)$ arising from thir Lagrangian finite difference description of the flow field.

For the finite difference approximation of (3.22) to (3.25), a three step procedure very similar to the one described in $§ 3.2 .2$ is used. The only modifications introduced by the use of a moving grid are the replacement of the constant $\Delta x$ by a variable mesh size $\Delta x_{1}$ and the displacement of the computation grid calculated from the finte difference approximation of (3.26):

$$
x_{\imath}^{j+1}=x_{\imath}^{j}+\Delta t\left[\theta \bar{u}_{\imath}^{j+1}+(1-\theta) \bar{u}_{\mathfrak{\imath}}^{\jmath}\right]
$$

where $0 \leq 0 \leq 1$

\subsection{Initial and boundary conditions}

As mentioned before, the numerical solution of the governing equations requires the hnowledge of initial and boundary conditions for $\eta$ and $\bar{u}$. Some of these conditions are obvious and aise directly from the physics of the problem while others must be dermed indirectly by using the governing equations.

\subsubsection{Initial conditions}

In all the problems to be treated in $\S 4$, the initial conditions correspond to still water. Thus

$$
\eta(x, 0)=0
$$


and

$$
\bar{u}(x, 0)=0
$$

for all $x$. In finite difference form, the conditions (3.28) and (3.29) berome

$$
\begin{aligned}
& \eta_{\imath}^{0}=0 \\
& \bar{u}_{\mathfrak{\imath}}^{0}=0
\end{aligned}
$$

for $i=0,1,2, \ldots, N$.

\subsubsection{Boundary conditions}

In problems where the flow field is bounded by a vertical wall at $x=x_{0}$, wo have the following boundary condition for the depth-averaged fluid velocity

$$
\bar{u}\left(x_{0}, t\right)=\bar{u}_{w}(t)
$$

where $\bar{u}_{w}(t)$ is the horizontal velocity of the wall. In finite difference, we have

$$
\bar{u}_{0}^{J}=\bar{u}_{w}\left(t_{\jmath}\right)
$$

If the wall is not moving, $\bar{u}_{w}=0$ and

$$
\bar{u}_{0}^{3}=0
$$

In all the cases to be treated in $\S 4$, the flow field is bounded at all times by a fixcer vertical wall at the downstream end $x=x_{N}$ such that

$$
\bar{u}_{N}^{J}=0
$$

for $\jmath=0,1,2, \ldots, P$.

The free surface position $\eta$ at $x=x_{0}$ and $x=x_{N}$ is unknown for $t>0$. 'Thrir. fore, unlike the case of the velocity, simple Dirichlet boundary conditions cannot, be deduced directly. However, by combining the velocity boundary conditions with the depth-averaged momentum equation (2.34), we can derive boundary condituons of 
the Neumann type for $\eta$. For example if $\bar{u}\left(x_{0}, t\right)=0$, equation (2.34) evaluated at $x=x_{0}$ becomes

$$
\eta_{x}\left(x_{0}, t\right)=\left[h h_{x t} \bar{u}_{x}+\frac{h}{2} h_{t} \bar{u}_{x x}+\frac{h}{2} h_{t x t}\right]_{x=x_{0}} .
$$

Equation (3 36) can be further simplified in cases where the bed acceleration at $x=x_{0}$ is equal to zero:

$$
\eta_{x}\left(x_{0}, t\right)=\left[h h_{x t} \bar{u}_{x}+\frac{h}{2} h_{t} \bar{u}_{x x}\right]_{x=x_{0}} .
$$

Furthermore, if the bed slope is constant at $x=x_{0}$, we have

$$
\eta_{x}\left(x_{0}, t\right)=\left[\frac{h}{2} h_{t} \bar{u}_{x x}\right]_{x=x_{0}}
$$

and funally, for a non moving bed at $x=x_{0}$ :

$$
\eta_{x}\left(x_{0}, t\right)=0
$$

If however, $\bar{u}\left(x_{0}, t\right)=\bar{u}_{w}(t)$, for the case of the non moving bed at $x=x_{0}$, we can write:

$$
\eta_{x}\left(x_{0}, t\right)=-\frac{d \bar{u}_{w}(t)}{d t} .
$$

At the downstream end $x=x_{N}$, we will always have :

$$
\eta_{x}\left(x_{N}, t\right)=0
$$

The finite difference approximations of equations (3.36)-(3.41) will be used together with the other initial and boundary conditions in the numerical solution of the various problems presented in $\S 4$.

\subsection{The Flux Corrected Transport (FCT) method}

In $\S 4$, specific moving bed problems will be solved with the help of the finite difference approximations derived in $\S 3.2$ for the governing equations. In most cases, these bed motions will be such that strong deformations of the free surface position will occur over relatively short distances. In other words, considerable variations in $\eta$ (and consequently on $\bar{u}$ ) will take place over a very small number of grid points. 
It is a well known fact that in finite defference methods the treatment of solutions with sharp gradients may lead to accuracy and/or stability publems. Standurd techniques available to circumvent these difficulties inchude gunl refimment in the regions of sharp gradients and the introduction of artiticial vincosity. Howerer, one of the most effective technque is certainly the so-called Flux Corrected Trampont (FCT) method which was initially developped hy Boris \& Book $(1973,1975,1976)$ for the numerical treatment of shock waves in compressible flows. In vew of applying it to the treatment of the problems presented in $\$ 4$, wo now give a brief summary of that method.

The first important thing to note is that the FCT nethod is not a nument cal scheme in itself but rather a procedure for handling ste(p) gradients solutums calculated by means of standatd mumerical techniques such an the finte defference method. We here follow the general detintion of FCT given by Zalesalk (1979).

We start by considering the following conser vation c(puation

$$
u_{t}+f_{s}=0
$$

where $w$ and $f$ are both functions of the independent varbalbles $r$ and $t$. A linite. difference approximation to $w$ is sald to be in conservative on "flux" fonm if it can be written as :

$$
w_{1}^{j+1}=w_{1}^{J}-\Delta x_{1}^{-1}\left[F_{1+(1 / 2)}-F_{1-(1 / 2)}\right]
$$

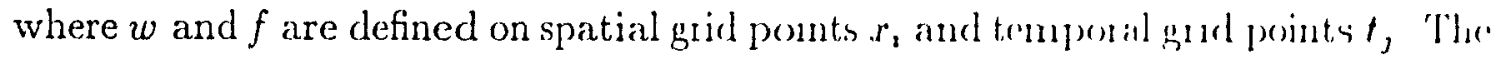
spatial mesh size is given by $\Delta x_{1}=\frac{1}{2}\left(r_{t+1}-r_{t-1}\right)$ The tarm $F_{1+(1 / 2)}$ ind $F_{t-11 / 2)}$

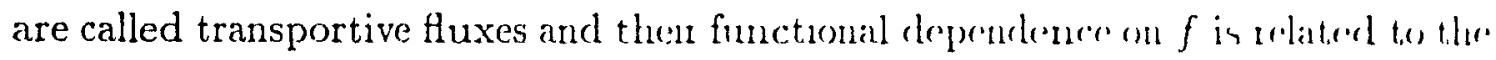
specific finite difference scheme which is used to approximatre (3.12). For cximulle, in the case of an Eulerian (fixed grid) Crank-Nicolson srlecmen, wo wonld hitve:

$$
F_{i+(1 / 2)}=\frac{\Delta t}{4}\left[f_{1+1}^{j+1}+f_{i+1}^{\jmath}\right]
$$

It can be shown (Boris \& Book 1973) that in the segions of sterep gradiernts in $w$, finite difference approximations to (3.42) may suffer fosm $f_{1}$ we prossible problums 
First, if the particular integration scheme which is used is of high order (order 2 or above, e.g. leapfrog, Crank-Nicolson, ...), the solution $w$ will show dispersive 'ripples'. On the other hand, if a low order scheme (e.g. Lax-Friedrichs, donor coll, .) is used, the solution will not be affected by ripples but will suffer from excessive numerical diffusion. A similar result is also obtained if a zeroth order artificial viscosity is added to a high order scheme.

The basic idea behind FCT is the combination of the best aspects of the above two schemes: the accuracy of a high order scheme coupled with the diffusive properties of a low order scheme. The originality of the method is that it constructs a net transportive flux point by point as a weighted average of a flux computed by a low order scheme and a flux computed by a high order scheme. This weighting procedure favors the use of the high order flux except in regions of steep gradients where the arising dispersive 'ripples' are attenuated by increasing the contribution of the low order flux. Therefore FCT can be thought of as a selective application of numerical diffusion which is limited to steep regions of the solution domain in which that diffusion is most needed. That constitute a major advantage over most artificial viscosity methods in which the solution is usually diffused uniformly over the whole domain.

The formal FCT procedure can be defined as follows (Zalesak 1979) :

1. Compute $F_{1+(1 / 2)}^{H}$, the transportive flux as given by a high order scheme.

2. Compute $F_{1+(1 / 2)}^{L}$, the transportive flux as given by a low order scheme guaranteed to give monotonic (ripple-free) solutions.

3. Define the "antidifusive flux":

$$
A_{i+(1 / 2)} \equiv F_{i+(1 / 2)}^{H}-F_{i+(1 / 2)}^{L} \text {. }
$$

4. Compute the updated low order ("transported and diffused") solution:

$$
w_{i}^{t d}=w_{1}^{3}-\Delta x_{1}^{-1}\left[F_{i+(1 / 2)}^{L}-F_{i-(1 / 2)}^{L}\right]
$$


5. Limit the antidiffusive flux by using

$$
A_{i+(1 / 2)}^{c}=C_{1+(1 / 2)} A_{1+(1 / 2)} \text { with } G \leq C_{1+(1 / 2)} \leq 1
$$

in a manner such that $u^{3+1}$ as computed in (6) is free of extrema not found in $w^{t d}$ or $w^{w}$.

6. Compute the final solution by using the limited antiduffusive fluxes calculated in (5):

$$
w_{\imath}^{j+1}=w_{\imath}^{t d}-\Delta x_{i}^{-1}\left[A_{\imath+(1 / 2)}^{c}-A_{1-(1 / 2)}^{c}\right] .
$$

The crucial part of the FCT method is obviously the choice of an appropriate selection rule for limiting the antidiffusive fluxes in (5). This important flux limitation or "flux correction" step determines the respective contribution of the dispersive (high orden) and diffusive (low order) effects in the final solution.

For the problems of $\S 4$, the simple FCT algorithm proposed by Peyret \& Taylor (1983) will be used to improve the numerical solution of the depth-averaged contmuily equation :

1. Compute $\eta_{t}^{j+1}$ by the high order method described in $\$ 3.2$.

2. Compute a low order (transported and diffused) estimation $\bar{\eta}_{t}^{j+1}$ of the solution by using

$$
\bar{\eta}_{i}^{j+1}=\eta_{i}^{j+1}+\sigma\left[\eta_{t+1}^{j+1}-2 \eta_{1}^{j+1}+\eta_{1-1}^{j+1}\right] .
$$

where $\sigma$ is an artificial viscosity coefficient.

3. Compute the limited antidiffusive flux $A_{i+(1 / 2)}^{c}$ by using the following selen tion rule :

$$
A_{t+(1 / 2)}^{c}=S \max \left[0, \min \left(S \Delta_{t-(1 / 2)},\left|A_{\imath+(1 / 2)}^{1}\right|, S \Delta_{t+(3 / 2)}\right)\right]
$$

with

$$
\begin{aligned}
A_{t+(1 / 2)}^{1} & =\frac{1}{8}\left(\eta_{t+1}^{j+1}-\eta_{\mathfrak{t}}^{\jmath+1}\right) \\
S & =\operatorname{sign}\left(\Delta_{t+(1 / 2)}\right) \\
\Delta_{t+(1 / 2)} & =\bar{\eta}_{\imath+1}^{\jmath+1}-\bar{\eta}_{\mathrm{t}}^{j+1} .
\end{aligned}
$$


4 Compute the final solution

$$
\eta_{1}^{j+1}=\bar{\eta}_{1}^{j+1}-\left(A_{1+(1 / 2)}^{c}-A_{1-(1 / 2)}^{c}\right)
$$

Although it may not be obvious at first sight, the above algorithm constitutes a specific example of the application of the formal procedure described previously. 


\section{COMPUTATIONS AND EXPERIMENTS}

As pointed out in $\S 1$, there are numerous instances in which water waves can be generated in a water body by the motion of the underlying bed. Natural phenomena such as landslides, rockfalls and submarine earthquakes as well as underwater voludule eruptions or explosions are a few examples of the many possible sources of such waw formation. One of the important questions arising in the modelling (pliysical or numerical) of these problems lies in the proper choice of a wave generation medhammu which must be as close as possible to reality.

In problems of water waves generated in a channel by underwater earthpushes (tsunamis) or explosions, the wave generation mechanism usually consists in the sudden vertical upthrust of a segment of the bed (Kranzer \& Keller 1959); Hwang \& Divoky 1970; Hammack 1973). This disturbed segment remains under water it all times.

Waves generated by landslides or other types of sliding material arc oftell simmlated by two distinct wave generation mechanisms: the horizontal motion of a voitual wall and the vertical fall of a box (Wiegel et al. 1970; Noda 1970; Das \& Wiegel 197:). In both cases, the disturbed segment is located at the end of a channel and intersects the water surface. The logic behind this type of modelling is that the actual wave generation mechanism probably consists of a mixed horizontal-vertical type of motum which lies somwhere between the fully horizontal and vertical generation modes.

In this section, we present five cases of wave generation by a moving low and describe their corresponding experimental and numerical models.

\subsection{Types of bed motion considered}

The present study was made in collaboration with workers at the Laboratosy of Hydraulics, Hydrology and Glaciology (VAW) of the Swiss Federal Institute of 'Tie.hnology (ETH) in Zurich.

The five following numerical simulations were conceived in conjunction with iden- 
tical laboratory experiments in order to permit a direct comparison of computational and experimental results. The experiments were performed at VAW in 1987-88 by Johannes Sander under the direction of Drs. K.Hutter and D.Vischer.

\subsubsection{Moving wall}

Formally speaking, the horizontal motion of a vertical wall at the end of a long channel does not constitute an example of wave generation by a moving bed. Indeed, the modelling of that problem by the Boussinesq theory does not require the use of the general equation (2.34) but can rather be handled by the simple Boussinesq equation (2.39) for waves over a flat bed with appropriate boundary conditions to morlel the moving wall.

However, we will still treat this case for two important reasons. Firstly, it constitutes an obvious starting point in the modelling of landslide generated waves. Secondly, that representation not only gives a rough simulation of the actual problem but it also can be easily implemented in an experimental or numerical model.

Figure 4.1 a shows that arrangement which from now on will be referred to as the 'moving wall'. The wave generation simply arises from the displacement of the wall over a distance $d$ with a velocity $\bar{u}_{w}(t)$ during a time interval $0 \leq t \leq t_{f}$.

\subsubsection{Submerged wedge}

The second wave generator which will be considered consists of the horizontal displacement of an inclined plate of constant slope $m$ at the end of a channel (Figure 4.1b) At $t=0$, the channel depth is constant. This wedge shape then moves over a distance $d$ into the channel with an horizontal velocity $\bar{u}_{w}(t)$. The total duration $t_{f}$ of the displacement is always chosen such that the resulting bed deformation does not reach the level of the undisturbed free surface. For that reason, we will refer to this wave generating device as the 'submerged wedge'. The fact that the moving bed never intersects the undisturbed free surface greatly simplifies the numerical solution of the problem by avoiding the treatment of a moving waterline. 


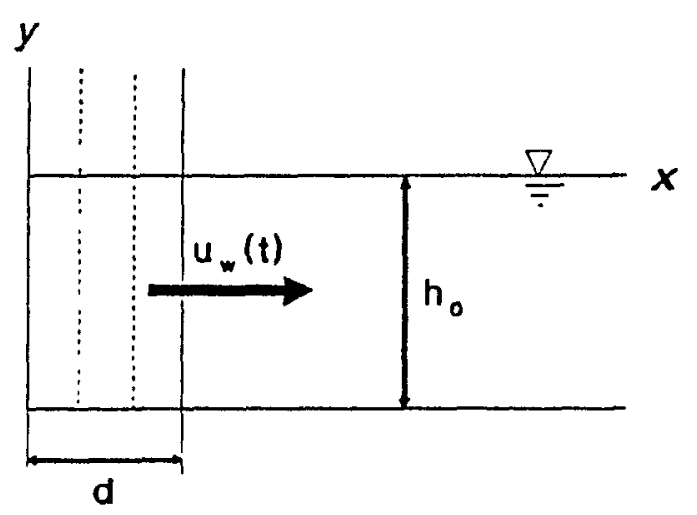

a) Moving wall

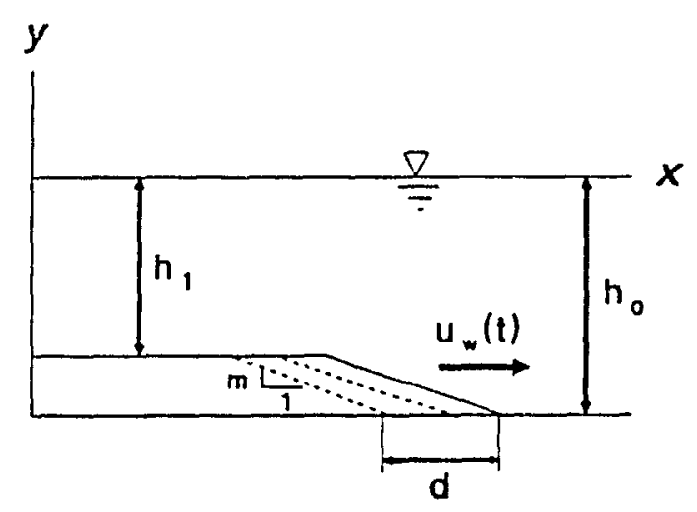

c) Moving shelf

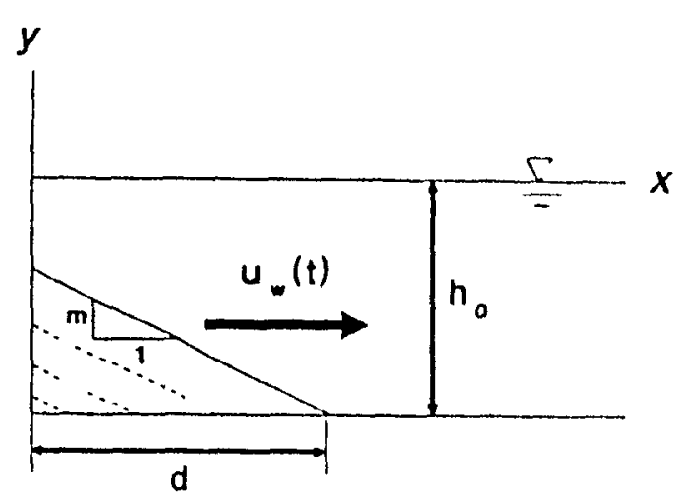

b) Submerged werlge

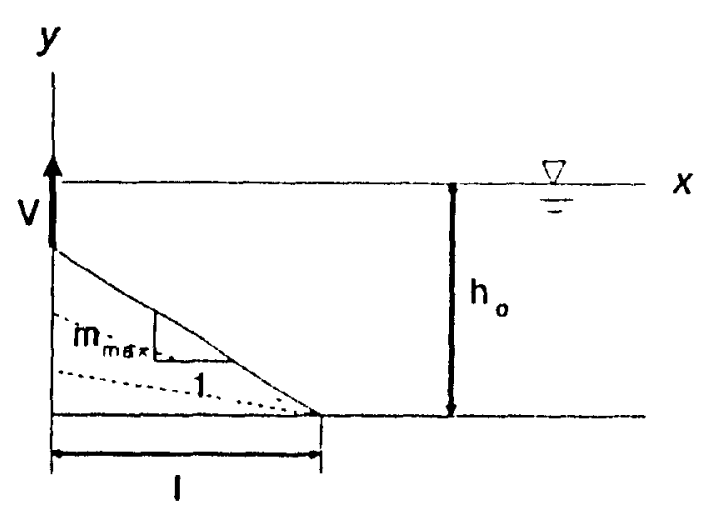

d) Rotating plate

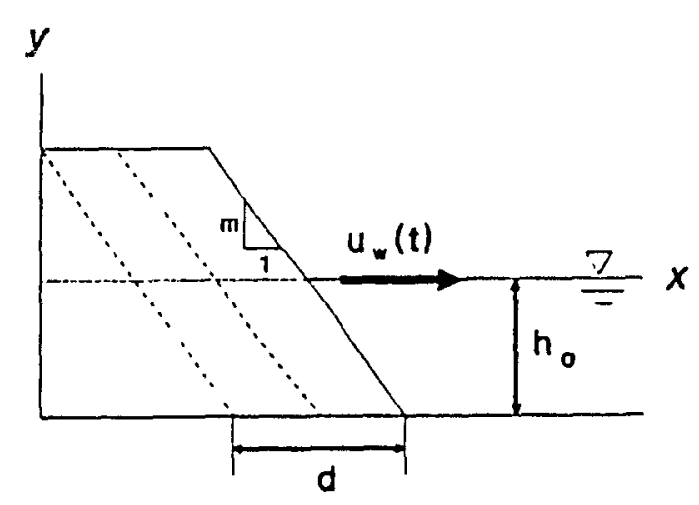

e) Moving wedge

Figure 4.1: Types of bed motion considered. 
Compared to the moving wall, the submerged wedge certainly constitutes a better representation of the entry of a landslide in water. The model was inspired by the probable configuration of a mass of granular material which is inclined at its angle of repose after entering the water.

\subsubsection{Moving shelf}

This wave generating device constitutes an extension of the previous case, the only differences being that the wedge now posseses a predefined height less than the undisturbed water depth and that its motion takes place some distance away from the upstream end of the channel (Figure 4.1c). The depth above the shelf is denoted by $h_{1}$. The resulting arrangment is referred to as the 'moving shelf'. This model can probably simulate the advance of a long strip of material along the bed of the channel. Contrary to the two previous wave generating devices, the moving shelf could probably be useful in the modelling of tsunamis.

The model was conceived as an extension of the shelf topography which was often used in the study of solitary wave propagation (Madsen \& Mei 1969; Johnson 1972; Helfrich \& Melville 1986).

\subsubsection{Rotating plate}

'The 'rotating plate' wave generating device consists of a plate which is rotated about a fixed location $x=l$ at the bottom of the channel (Figure 4.1d). The plate initially concides with the bed of the channel and is then lifted with a constant vertical velocity $V$ at $x=0$ until the slope reaches a maximum value $m_{\max }$ at $t=t_{f}$ which is always chosen such that the deformed bed does not go beyond the undisturbed free surface level. Unlike the previous wave generators, the rotating plate does not involve a lateral translation of the wave generating device in the $x$-direction. Furthermore, Its iesulting motion implies a strong vertical component.

The rotating plate could well represent the accumulation at the bottom of the channel of a granular mass which does not travel laterally but rather builds up into 
a sloping heap.

\subsubsection{Moving wedge}

Compared to the four other wave generating devices, the 'moving wedge' (Figure 4.1e) probably constitutes the best, model for landslide generated waves. It molves the motion of an inclined wail spanning accross the whole water depth. The hor zontal bed velocity is denoted by $\bar{u}_{w}(t)$ and its constant slope by $m$. The resultung bed deformation is similar to the case of the submerged wedge excopt that it now intersects the free surface. As pointed in $\$ 3.2 .2$, the moving wedge nodel mtioduces the difficulty associated with the numerical treatment of the constantly moving pont of intersection between the bed and the free sulface.

\subsection{Experimental set-up}

As mentioned earlier, the five wave generating devices described above wese modellerl experimentally at the Laboratory of Hydraulics, Hydrology and Glaciology (VAIV) in Zurich, Switzerland (Sander 1988).

The channel used for the experiments was $16 \mathrm{~m}$ long and had a crows section of $0.3 \mathrm{~m} \times 0.3 \mathrm{~m}$. The bottom and back walls were made of $1 \mathrm{~cm}$ thick dark grev PVC sheets while the front was made of a $1 \mathrm{~cm}$ thuck plexiglass wall to permut durect observation of the propagated waves. Two types of wave generating mechanisms wan used.

\subsubsection{Rotating plate}

For the rotating plate generator, a flat plate 30 to $50 \mathrm{~cm}$ long located at the upstirim end of the channel was hinged to a cylindrical bearing at the bed. The plate was rotated around that hinge by the vertical traction of an electrical motor. At $x=($, the water was bounded by a vertical plate which was free to move in the vertir al direction as the rotating plate was set in motion. 


\subsubsection{Lateral displacements}

For all the remaining wave generating devices, a common basic mechanism was used. A piston driven by an electrical stepping motor was installed at the upstream end of the channel. A gearing with cross-like slot guides was used to transform the rotation of the motor into the horizontal translation of two straight parallel bars. Different piston geometries were attached to the extremity of these bars to make up the various wave: generating devices.

Fon the moving wall, a simple vertical plate was used. In the case of the moving werlge, it, was replared by inclined plates with slopes of 15,30 or $45^{\circ}$. The submerged wedge generator was obtained.by placing a moveable vertical partition above the mclmed plate at $x=0$. For the moving shelf, a long, shallow rectangular box was inst,alled in front of the vertical wall piston. Again the water was bounded at $x=0$ by a vertical partition

The electrical stepping motor turns at a constant frequency. By neglecting its initisl acceleration and final deceleration, it can be shown that the rotation of the motor gives rise to an horizontal piston velocity which has a sinusoidal variation in time (Sander 1988). The piston velocity can be expressed as :

$$
\bar{u}_{w}(t)=v^{*} \sin \left\{\pi\left[\tau_{1}+\frac{\left(\tau_{2}-\tau_{1}\right)}{t_{f}} t\right]\right\}
$$

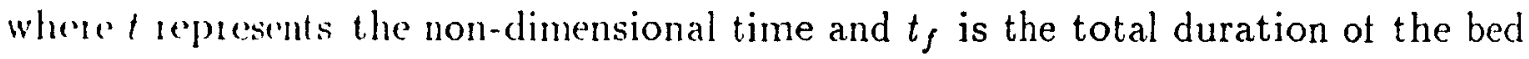
mot 1on. 'The' maximum piston velocity is denoted by $v^{*}$. The parameters $\tau_{1}$ and $T_{2}$ depend on the specific wave generating device which is used. In all experiments, $u_{w}(t)$ is non-negdtive at all times (i.e. no pulsating motion of the piston). For the moving wall, moving shelf and moving wedge, we always have $\tau_{1}=0$ and $\tau_{2}=1$. For th" submerged wedge, values of $\tau_{1}=\frac{1}{4}, \frac{1}{3}$ and $\tau_{2}=\frac{3}{4}, \frac{2}{3}$ are also used.

The tesulting horizontal displacement $d$ of the wave generating device was always predefined in each experiment. By integration of (4.1),

$$
d=\int_{0}^{t_{f}} \bar{u}_{w}(t) d t=v^{*} \int_{0}^{t_{f}} \sin \left\{\frac{\pi}{t_{f}}\left[t_{f} \tau_{1}+\left(\tau_{2}-\tau_{1}\right) t\right]\right\} d t
$$


from which we can deduce the following expression for the total duation of the hed motion :

$$
t_{f}=\frac{\pi\left(\tau_{2}-T_{1}\right) d}{v^{*}\left(\cos \pi \tau_{1}-\cos \pi \tau_{2}\right)}
$$

Equation (4.2) will be useful in the development of the computer models which will be presented in the next subsection.

\subsection{Computer models}

Five computer programs were developed in order to model the various cascen of willm induced by a moving bed described in $\$ 4.1$. We now give an overview of these pon grams' general characteristics and a description of their source code.

\subsubsection{General description}

All the computations were performed on IBM PC' or compatibles computers of the $\mathrm{XT}$ and AT type.

The programs were edited, compiled and run with the help of the TIIRBB() PAS CAL package (versions 3.0 and 4.0). The graphical output was produced by PL()'T. CALL 1.0.

Depending on the extent of the solution domain, the total computation tume fon each problem rülged from 3 to 10 hours.

The source codes of the five programs are listed in $\Lambda_{\text {ppendix }}(;$ along with the definition of the variables and of the constants used to specify the mithal paramelom of the problems. Table 4.1. gives the name of these programs and the wave gensmatme, mechanisms to which they correspond. All these programs have the same main structure, which can be divided in 3 basic parts.

A first set of procedures specifies the initial and boundary conditions for the dependent variables $\eta$ and $\bar{u}$. The initial conditions always correspond to a still water surface and are given by equations (3.30) and (3.31). The boundary comditum depend on the geometry and the type of displacement of each particular problem (sow 
$\{3.3 .32)$.

\begin{tabular}{|l|l|}
\hline \hline Type of wave generation & Name of program \\
\hline Moving wall & WALL \\
Submerged wedge & SUBWED \\
Moving shelf & SHELF \\
Rotating plate & ROPLATE \\
Moving wedge & WEDGE \\
\hline
\end{tabular}

Thble 4.1. Types of bed motion considered and corresponding computer programs

A second group of procedures insures the proper mathematical modelling of the evolution $h(x, t)$ of the moving bed. That part of the program is very important since the bed displacement history directly influences the characteristics of the resulting wise profile

The core of the programs involves the application of the numerical methods of $\S 3$ 10 the solution of the governing equations. This segment of the programs therefore pertorms the actual calculation of $\eta$ and $\bar{u}$ resulting from a known bed motion with appoptiate initial and boundary conditions

For the rexsons already pointed out in $\$ 3.2 .1$, both the Eulerian and the Lagranglan fimte difference schemes are used. The Lagrangian scheme is applied to the moving wall (progtam W'ALL) and the moving wedge problems (program WEDGE), which anc the two cases involving a point of intersection between the water surface and the wave gencrating device. The treatment of the three other cases (programs SUBUWED, SIIELF, ROPLATE) relies on Eulerian schemes.

In the case of Lagrangian computations, a fourth set of procedures was developed for the task of reallocating the moving spatial grid points to their new location at every tume step.

In orde' to droid possible accuracy or stability problems, the FCT scheme de- 
- scribed in $\$ 3.4$ was implemented in each program to eliminate non-physical ripples in the calculated wave profiles. The scheme proved to be necessary in all the prob lems treated except for the moving wall where a satusfactory solution was obtanned whithout the use of FCT.

We now proceed to a separate description of each program.

\subsubsection{Program WALL}

The WALL program models the moving wall problem. It is listed in Appendix (' on pages $\mathrm{C}-2$ to $\mathrm{C}-\mathrm{T}$ The main program is rolatively short and relies on the nue ol various procedures.

The procedure 'Initial_Cond' creates an initial spatıal grid having a const.unt menh size ' $\mathrm{dx}$ '. It also specifies on each grid points $(\iota=0,1,2, \ldots, n)$ initial value's '(r/hal|l]' and ' $u l[i]$ ' for the free surface position and the depth-averaged velocity which com. spond to equations (3.30) and (3.31), i.e. fluid at 1 est.

The velocity boundary conditions are implemented by the proredure 'U_B, 'whul uses equations (3.33) and (3.35). The free surface boundary conditions are beded on equations (3.40) and (3.41) and calculated by the procedure 'Etha_BC'

The horizontal motion of the wall is modelled by the functions 'Vpiston' and 'Accpiston' which respectively give the velocity and acceleration of the wall at. any given time. The function 'Vpiston' is based on equation (4.1) with $\tau_{1}=0$ and $\tau_{2}=1$

To obtain the numerical solutions of the governing equations, the three step) scheme proposed in $\S 3.2$ is incor porated on thee distinct procerdures.

The procedure 'Continuity_Predictor' calculates a predicted value 'Ml, halld' (' $1,2, \ldots, n-1)$ for the free surface position using equation (.3 24) with the "old' values 'ul[l]' of the velocity field. The pocedure 'Momentum' solvex equatom (3.25) by using an implicit (Crank-Nicolson) finite difference scheme.

The updated velocity field ' $\mathrm{Q} 2[\imath]$ ' $(\imath=0,1,2, \ldots, n-1)$ is obtained as the: solutucm of a tridiagonal matrix system by the procedure: 'Tridiag'.

Finally, the procedure 'Continuity_Corrector' also solves (324) but uses the value's 
'u2 $2[2]$ ' of the updated velocity field.

At rach time step, the updated location ' $\mathrm{x} 2[\mathrm{i}]$ ' of the moving grid points is determined by means of two procedures : 'Approx-Interfaces' and 'Interfaces'. The first one gives the approximate location of the grid points (or fluid interfaces) as given by equation (327) with $\theta=0$. The procedure 'Interfaces' is used after the 'Momentum' procedure and solves equation (3.24) with $\theta=1 / 2$.

\subsubsection{Program SUBWED}

This program uses an Eulerian finite-difference scheme to model the waves generated by a moving, submerged wedge. It is listed on pages $\mathrm{C}-8$ to $\mathrm{C}-17$ of Appendix $\mathrm{C}$.

The main program begins by the imposition of initial conditions and velocity boundary conditions via the procedures 'Initial_Cond' and 'U.Bc'. The velocity boundany conditions (3.3.1) and (3.35) are used.

The free surface boundary conditions are handled by the procedures 'Etha_Bc_ Pred' and 'Ttha_Bc_Corr' in accordance with equations (3.38) and (3.41).

The evolution of the moving bed is treated by the procedures 'Bed_is_lloving' and 'Move_Bed' which are based on equation (4.1). The procedure 'Stationary_Bed' is used to specify the topography of the bed once its motion has stop.

The contunuty equation is again solved in two steps by the procedure "Contiunuty-Predictor' which uses the finite-differenre expression (3.18) and by 'Continuthy_concector' which uses (3.20).

'The updated velocity field ' $12[2]$ ' is calculated by the procedure 'Momentum' in acordance with the implucit (Crank-Nicolson) scheme presented in §3.2.1). To reduce the computation time, two distinct procedures are used separately for calculations duning the bed motion (procedure 'Momentum_Fxt') and after the bed has come to u'st (procedure 'Momentum_Fx'). Both procedures use the 'Tridiag' matrix solver.

The procedue FCT corrects the updated free surface position 'etha2[2]' by using the Flux Cortected Transport (FC,T) algorithm presented in $\S 3.4$. 


\subsubsection{Program SHELF}

This program modelling the generation of a wave by the motion of a shelf is listed in Appendix $\mathrm{C}$, pages $\mathrm{C}-18$ to $\mathrm{C}-27$.

The source code is almost identical to SUBWED. The only differences are in the specification of the bed motion and in the free surface boundary conditions collouluted by the procedure 'Etha_Bc'. The upstream and downstream boundaries nuw bu', consists of fixed vertical walls over a non-moving bed and the conditions (33.39) and (3.41) are used.

\subsubsection{Progran ROPLATE}

This program models the rotating plate problem described in 34.14 . 'The lusting of ROPLATE can be found on pp. C-28 to C-37. Again, this program closely resemblum the other two (SUBWED and SHELF) using an Eulerian scheme.

The main difference is now in the specification of the bed motion which dexen now imply an horizontal sinusoidal velocity as given by (4.1) but rather a vert tual pull of constant velocity (Figure 4.2a).

The upstream free surface boundary condition at is now given by e(fual.10n (:3 33)

\subsubsection{Program WEDGE}

This program (pp. C-38 to C-47) treats the moving wedge problem of \$.1.1.5 As mentioned before, it uses a Lagrangian finite difference scheme. It comstituture a gerll eralisation of the program WALL in which the complete governing equat.on!s (3.22) (3.23) are used instead of (3.24)-(3.25).

The upstream boundary condition, now consists in a laterally movmg pomt of zero depth. Instead of using the equations derived in $\$ 3.3 .2$, we simply express the free surface boundary condition at this point by the equation

$$
\eta\left(x_{0}, t\right)=-h\left(x_{0}, t\right)
$$

which is used in procedure 'Etha_Bc'. 
Furthermore, the upstream velocity boundary condition is now calculated by a simple extrapolation (procedure 'U_Bc_Corr') of the values of the velocity field at the grod points $\imath=1$ and 2 .

The morlelling of the moving bed is now done by the procedure 'Find_Depth'. Unlike the previous cases, that procedure allows the use of an interpolating polynomal in order to sinooth off the transition between the sloping segment and the flat prit of the bed (Figure 4.1e). The interpolating polynomial is given by (Pedersen \& (ijevik 198:3) :

$$
p(s)=\frac{1}{32}\left(s^{6}-5 s^{4}+15 s^{2}-16 s+5\right)
$$

whicese

$$
s=\frac{m}{l_{t}}\left(x_{i}-x_{b}\right)
$$

and

$$
\begin{aligned}
& x_{b}=\text { position of the foot of the wedge, } \\
& m=\text { slope of the wedge, } \\
& l_{t}=\text { required length of the smoothed transition. }
\end{aligned}
$$

Thus feature was adred in order to investigate the effect of the slope discontinuity on the araluation of the fitst order spatial derivative $\frac{\partial h}{\partial x}$ at $x=x_{b}$. 


\section{COMPARISON OF RESULTS}

Graphical outputs obtained from the five computer models described carles will nw» be presented and compared with corresponding experimental results.

\subsection{Moving wall}

Figure 5.1a gives a representation in the $x$ - $t$ plane of a wave generated by the latior.1 motion of a vertical wall. The wave profiles were computed with the program WiIII. based on the Lagrangian scheme described in \$3.2.3. The initial spatial ment $4, \ldots$ was set to $\Delta x=0.25$ and the temporal mesh size to $\Delta t=0.25$. The F('T algumlim was not used in the numerical solution. For the case shown, the molion of the wall was governed by equation (4.1) with $\tau_{1}=0, \tau_{2}=1$ and $v^{*}=0.2$. The wall moverl from $x=0$ to $x=1.67$ in a time $t_{f}=13.12$ as calculated from equation $(4.2)$. 'Th'. instantaneous position of the wall is indicated by a line which becomes pardllel to the. time axis for $t \geq 13.12$.

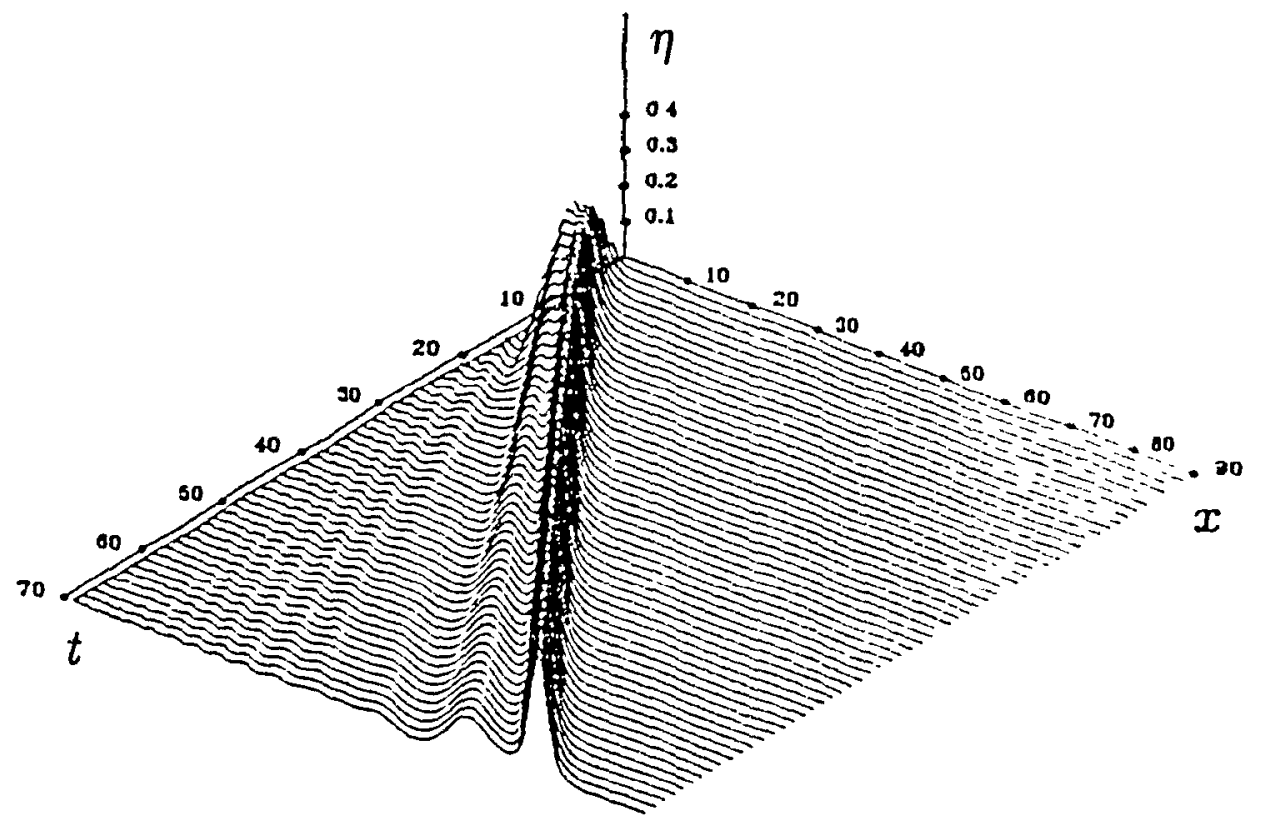

Figure 5.1a: Wave generated by a moving wall. 
As described in $\xi 2.1$, all the variables were non-dimensionalized according to a characteristic depth $h_{0}$ which was chosen to correspond to the free surface level at rest

Figure 5.1b shows a comparison of the above numerical output with results obtained from a channel experiment at VAW-ETH. The channel had an initial depth wrter depth of $h_{o}=15 \mathrm{~cm}$. The parameters governing the motion of the wall were all identical to the ones used in the computation. Results are presented as wave height recordings measured at eight locations along the channel. The experimental results arc repr'sented by a solid line and the computational ones by a dashed line.

It appears that the general agreement between computed and measured wave profiles is quite good. The free surface disturbance initially takes the form of a single lump) As it travels down the channel, the initial wave splits into a large leading wave followed by a train of smaller, dispersive oscrllations of decreasing amplitude. It can be shown (Sander 1988) that the leading wave matches almost exactly the solitary wave solution of the Boussinesq equation.It is worthwile noticing that the numerical model not only predicts the shape of that leading wave but it also gives a very good representation of the trailing dispersive waves.

However, there is a slight difference between computed and observed peak amplitudes Although the calculated peak of the initial disturbance (gauge 1) is very close to the measured value, it appears that further downst ream, the calculated amplitude is slightly larger. That small difference could probably be attributed to the neglect in the mathenatical model of the dissipation effects associated with the water viscosity and the wall friction in the channel.

The agreement between compuled and measured values indicates that our model call account properly for wave amplitudes in the order of $25 \%$ of the undisturbed water depth. Such values of the relative wave amplitude could probably not be treated usmg the 'small amplitude' theory. Figure D.1 in Appendix D shows a comparison between experimental and computational results for which a reasonable agreement was obtained even for a leading wave amplitude of more than 0.5 . 


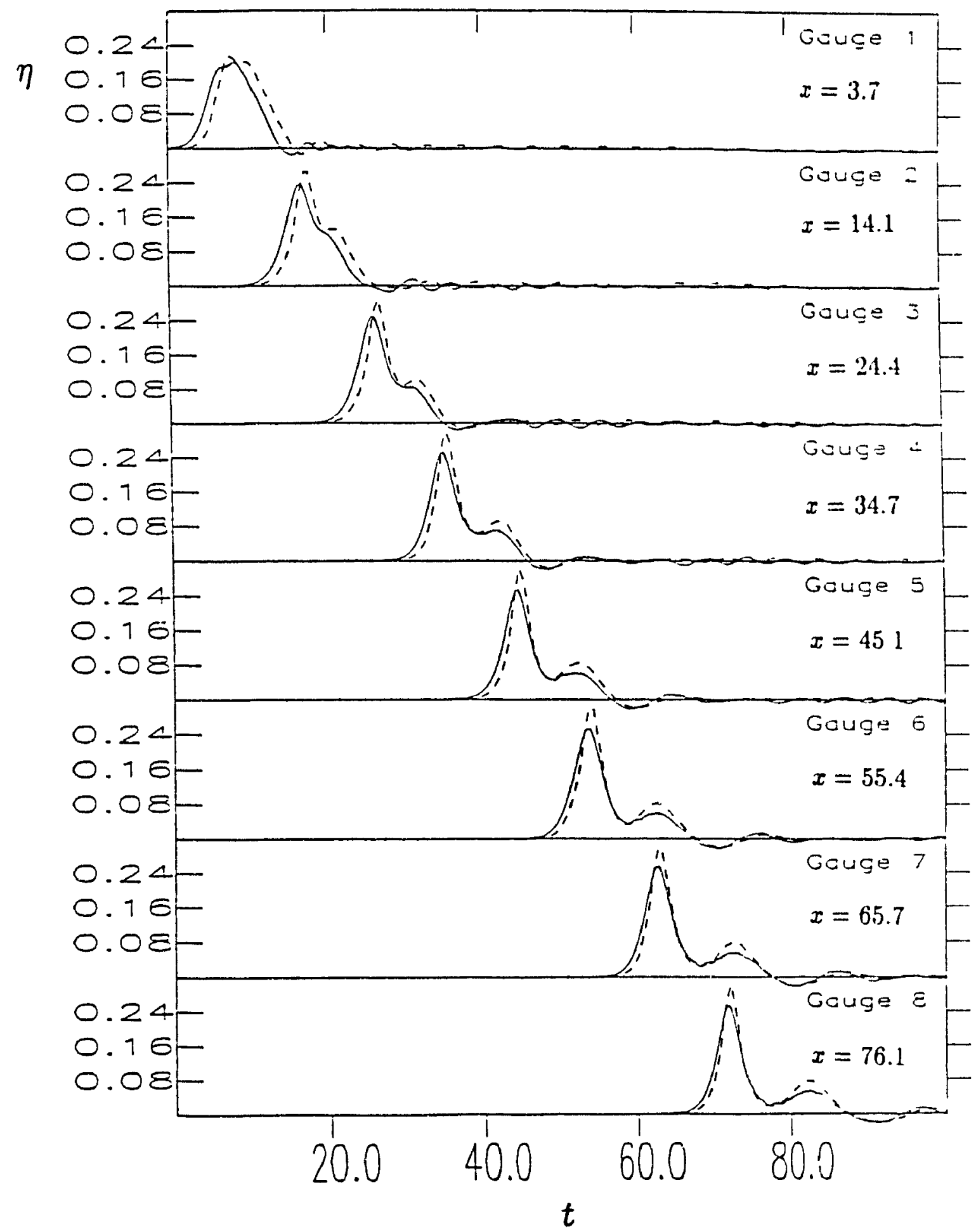

Figure 5.1b: Wave generated by a moving wall. Maximum horizontal veler ity of the wall $v^{*}=0.2$; total displacement $d=1.67$; duration of the motion $t_{\rho}=13.12$ Experiment -, compuration -.. 


\subsection{Submerged wedge}

The so-called 'submerged wedge' problem was the first moving bed problem to be treated in this study. For that reason, numerous computations were performed and supplementary results are presented in Appendix D.

Figure $5.2 \mathrm{a}$ represents the propagation of a wave generated by a moving submerged wedge having a slope of $0.268\left(15^{\circ}\right)$ and a sinusoidal velocity described by equation (41) with $\tau_{1}=0, \tau_{2}=1$ and $v^{*}=0.2$. The foot of the wedge moves from $x=0$ to $x=1.67$ in a time $t_{f}$ of 13.12 . The wave generation and propagation were computed by the program SUBIVED. The Eulerian scheme of $\$ 3.2 .2$ was used with a constant spat.ial mesh size of 0.25 and a temporal mesh size of 0.25 .

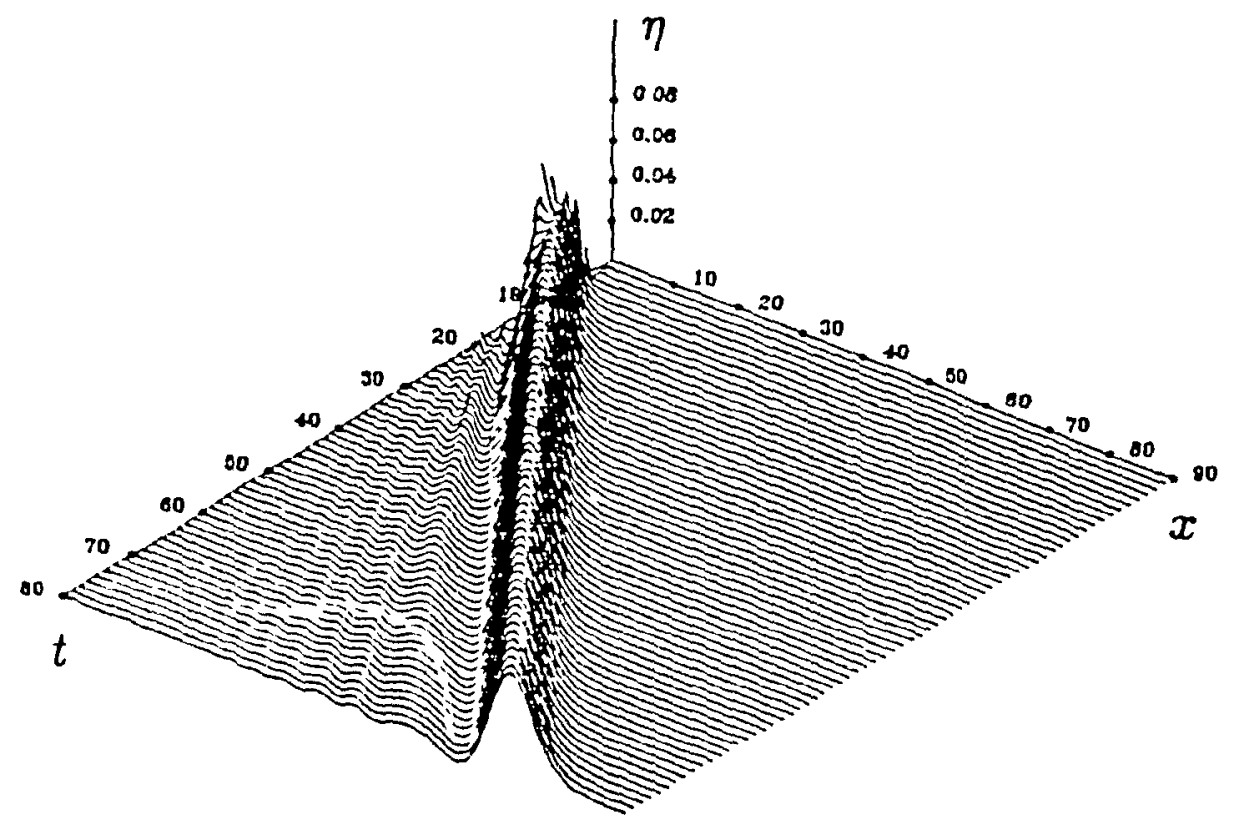

Figure 5.2a: Wave generated by a submerged wedge.

Unlike the case of the moving wall which we just described, the submerged wedge problem uses the FCT algorithm of $\S 3.4$. As already mentioned, the necessity of FCT In the subme،ged wedge problem (as well as in the three remaining cases involving a motion of the bed) can be explained by the fact that sharp variations in the bed clevation (and consequently on $\eta$ and $\bar{u}$ ) occur over only a few spatial grid points 
during the generation phase. That explains why FCT was not needed in the moving wall problem which was simply modelled by the Boussinesq system for waves over a flat bed with appropriate boundary conditions to handle the moving wall.

Figure D.2b in Apppendix D shows computational results obtained without the use of FCT for the bed motion conditions stated earlier. When compared to the sollul line of figure $5.2 \mathrm{~b}$ which was obtained from measurenents in a chaniel experiment. we see that the computed wave profiles of figure D.2b are unsatisfactory. $A$ tran of non-physical oscillations is trailing the leading wave. These 'wiggles' are chperialls important in wave profiles computed during the generation phase.

The results of a computation using the FCT algorithm with a diffusion combtaut $\sigma$ of 0.2 are shown in figure $5.2 \mathrm{a}$ and represented by a dashed line in figure 5.21) (these results are also shown on figure D.2a but with a different scale).

The agreement between computed and measured wave wave heights (Figure 5.2!) is even better than in the case of the moving wall. The calculated peak amplitudes now closely match the experimental values. Although the dissipative cifleck associated with the experiment are still probably present, it would seem that a con responding diffusion is introduced in the computed profiles by the use of I'('I'

Again, we observe that our model permits appropriate representation of both the leading wave and the trailing sequence of smaller dispersive waves. To reinfor.. that. statement, computations were carried out using the linearized long-wave "quations (1.4) and the shallow-water, finite-amplitude equations (1.2) in non-dimensiontal form (i.e. $g$ replaced by 1). The results are shown in Appendix D) in figures D).2i and I).21 We observe that both theories ate unable to predict the occurence of the thanling, dispersive waves. Furthermore, in both cases, the calculated leading wave is valud only for a short time. As it travels downstream, its amplitude becomes overestimated and, in the case of the finite-amplitude equations (Figure D.2d), it suffers from an excessive steepening. 


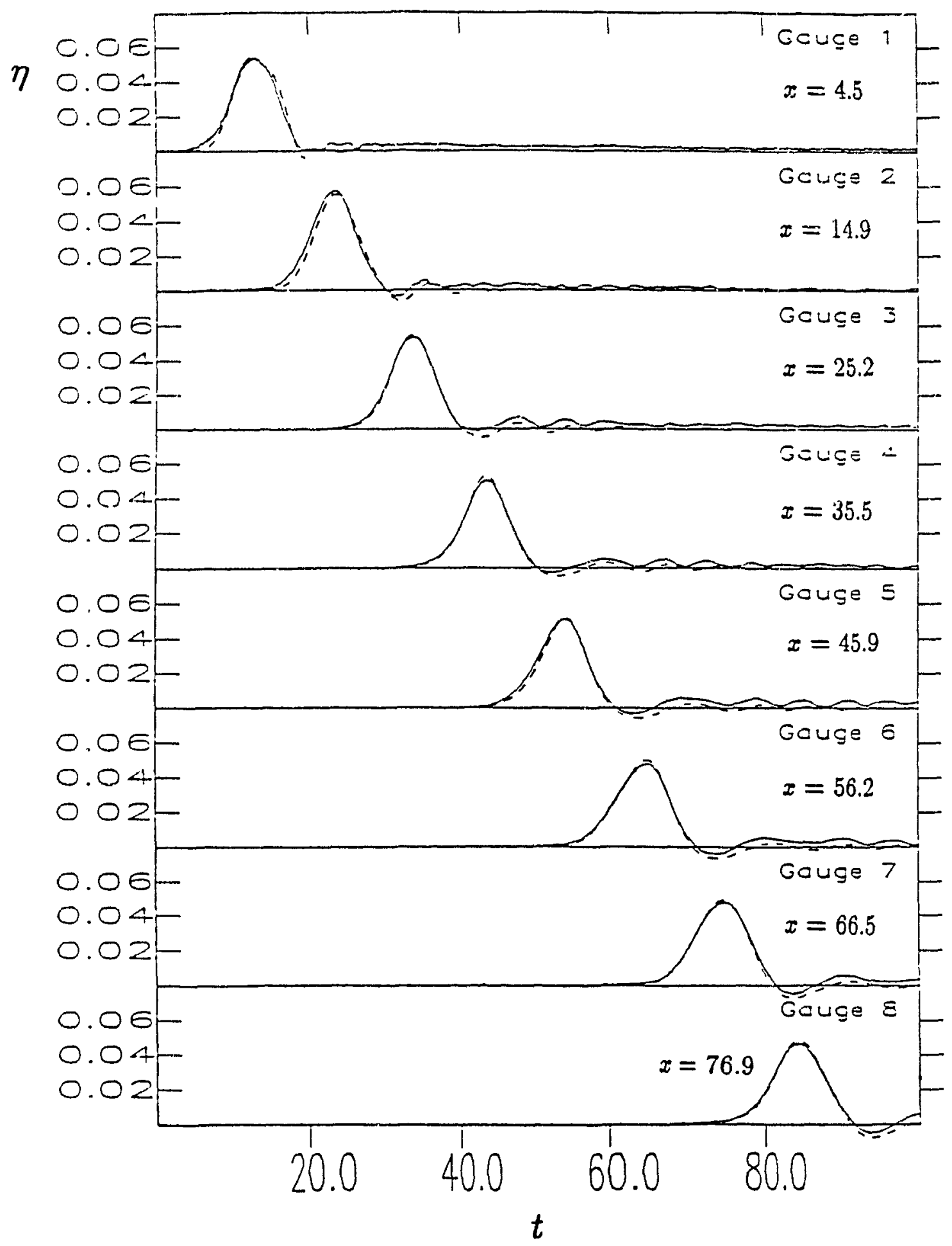

Figure 5.2b: Wave generated by a submerged wedge. Slope $m=0.268$; maximum horizontal velocity of the wedge $v^{*}=0.2$; total displacement $d=1.67$; duration of the bed motion $t_{f}=13.12$. Experiment - ,computation - - . 
In order to confirm the validity of our weakly nonlmear. dispersive approuch, the results of two other sirnulations are presented. Figures D.2e and I) af both involwe the . same displaced bed volume as the case we already described (Figure 1) an), The wate profiles of figure D.2e were ubtained by reduring the amplitude ${ }^{*}$ of the sinissoidal wedge velocity from 0.2 to 0.1 and by increasing the duration $t_{f}$ of the bed molwn from 13.12 to 26.23 in order to obtain the same displaced volume. Simularly, in tigule D. $2 f, v^{*}$ was kept at 0.2 but the slope was incieased from $0.268\left(15^{\circ}\right)$ to $0.577\left(30^{\circ}\right)$ thus reducing the motion time from 13.12 to 8.94 . The conclusion which can be drawn from comparison of figures D.2a, D.2e and D.2f is that the far field bethavem of the wave depends on the bed motion history and not only on its final displacement. This fact contradicts the conclusion which can be derived from the small-amplitude theory (Hunt 198s) and which states that, under the influence of dispersion, all waves created by the same resultant bed displacement should ultimately become identilu al

Supplementary results are presented on figures D.2g, D.2h and D $2 /$ to confirm the validity of the model.

\subsection{Moving shelf}

As mentioned in $\S 4.1 .3$, the 'moving shelf' wave generator is similar to the case of the submerged wedge. The interest of this wave generator lies in the fart that the initinl free surface disturbance is induced some distance away from the origin Them for. the moving shelf could probably be useful in the study of waves generated aw wy from the shoreline such as tsunamis.

Computations and corresponding experments were performed to modrl the popagation of a wave generated by the motion of a shelf having the following charactur istics:

- depth of water above the shelf $h_{1}=\frac{2}{3} h_{0}$ with $h_{0}=1.5 \mathrm{~cm}$ in the experiment,

- slope of the depth transition $m=0.577$,

- initial position of the top of the transition $=5$, 
- distance travelled by the shelf $d=1.67$,

- duration of mution $t_{f}=13.12$.

The Icsulting wave profile is shown in the $x$-t plane in figure 5.3a. The numerical results are compared with experimental values in figure $5.3 \mathrm{~b}$. The computations were performed with the program SHELF. The spatial and temporal mesh size were again set to 0 25. Flux Corrected Transport was used with $\sigma=0.2$.

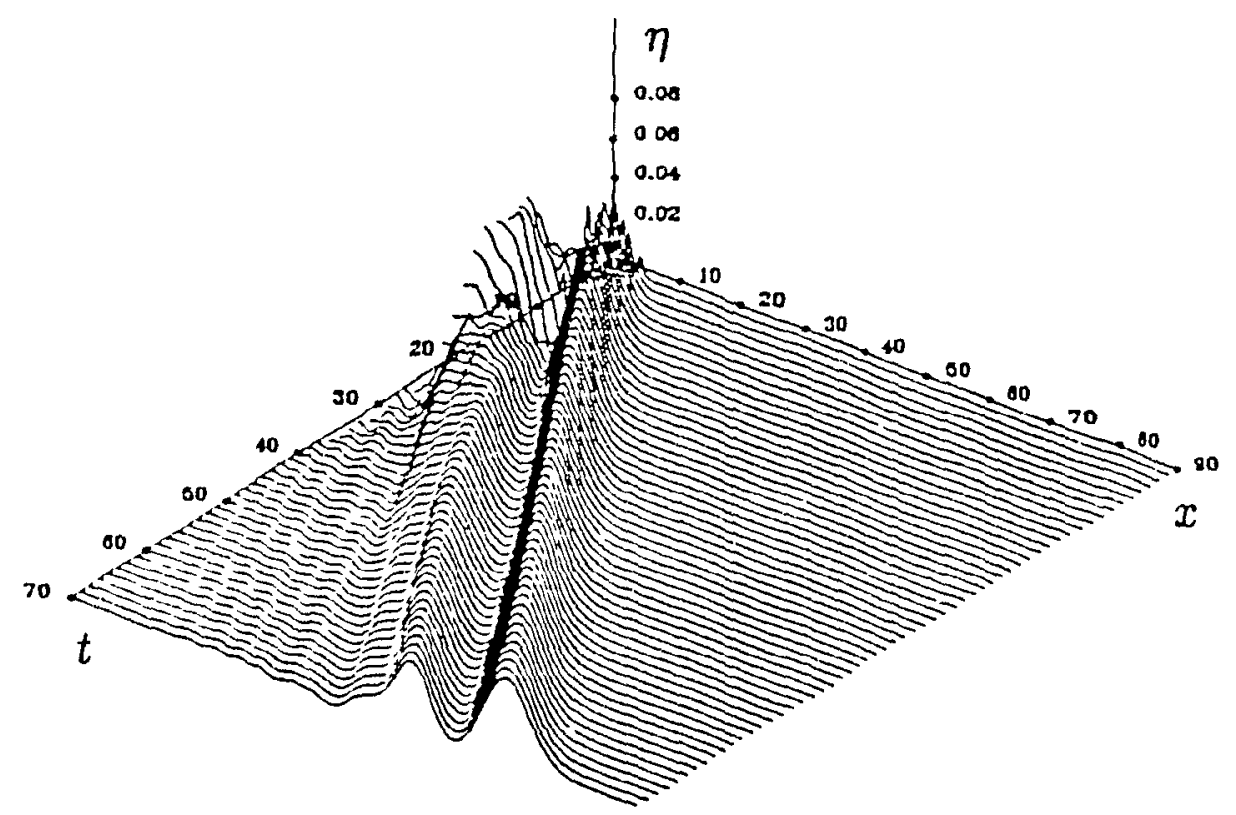

Figure 5.3a. Wave generated by a moving shelf.

The mut al disturbance is split up into a right running and a left running wave. Is it waches the origin, the left running wave is reflected and starts to trail the right tunnmg wave Figure 5.3b indicates a very good agreement between cmputed and experimental values.

The only observable difference is in the amplitude of the trough which follows the rellected a ave The calculated amplitude is slightly higher than the observed one. Howeser, as the increases, the numerical and experimental wave heights become chuil (se" sanges 3 to 6 ) 


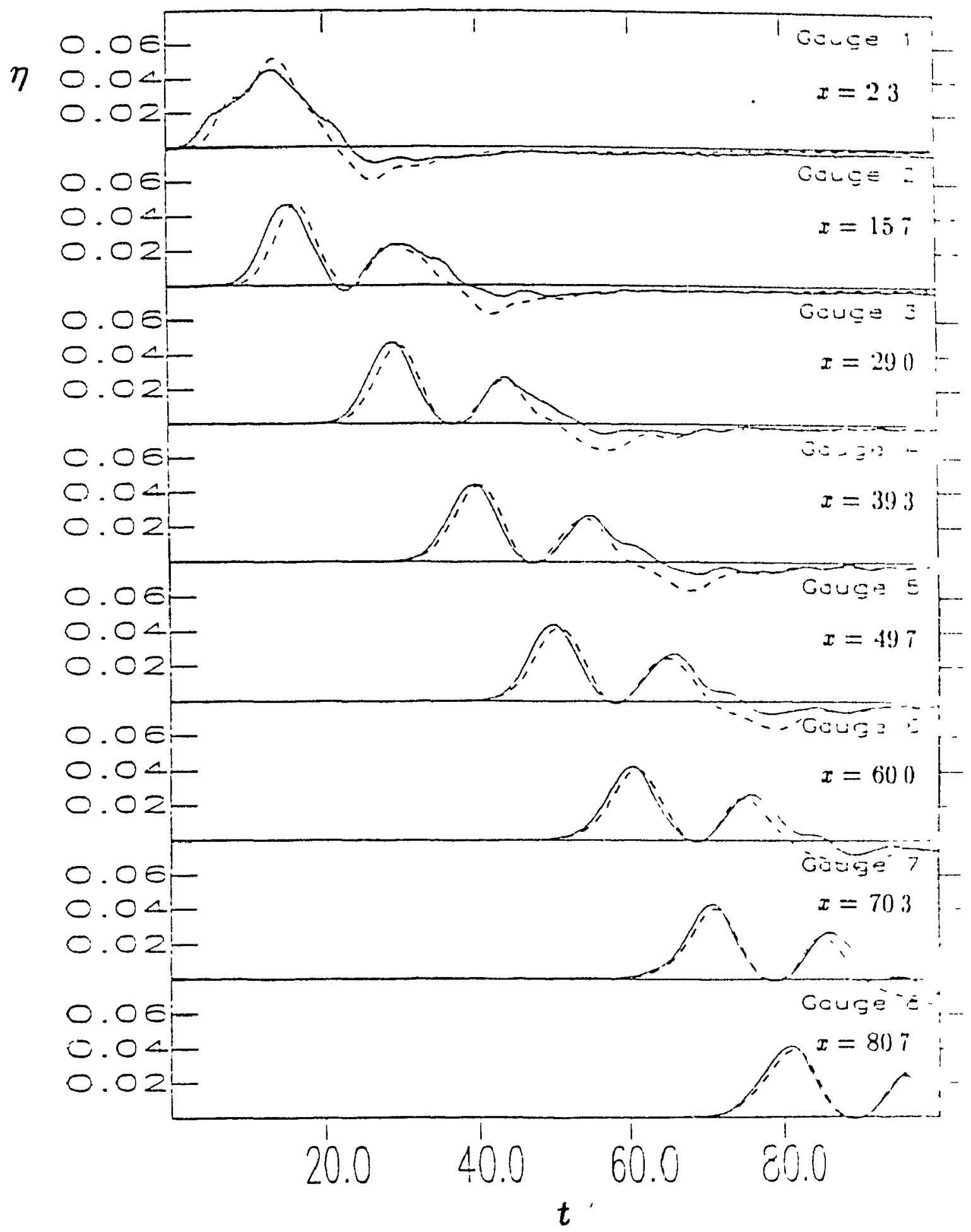

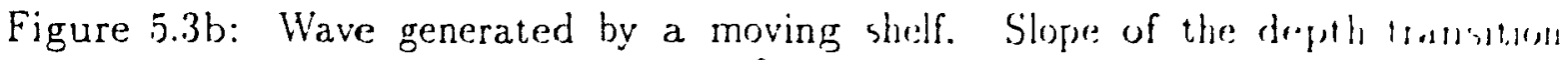
$m=0.57 i$; depth above the shelf $h_{1}=\frac{2}{3} h_{0}$, maxmum horizontal velis sty $10^{*}=112$ total displacement $d=1.67$; duration of the bed mot.on $t_{f}=1.3 .12 \mathrm{H.4.411}$ ment - computation -.. 


\subsection{Rotating plate}

The 'rotating plate' problem is the only one which does not involve a sinusoidal motion of the wave generator. The motion here simply consists of the rotation of a - Hlat plate resulting from a pull of constant velocity applied dt $x=0$.

The: program ROPLATE was used to calculate a numerical solution for the wave hinght $\eta$ The results ase shown in figure $5.4 \mathrm{ta}$ and compared with experimental data In figure 5. H) In both cases, the plate had a length $l=3.33$ and was rotated for $t_{f}=8$ with a veltical velocity $V=0.1$ at $x=0$

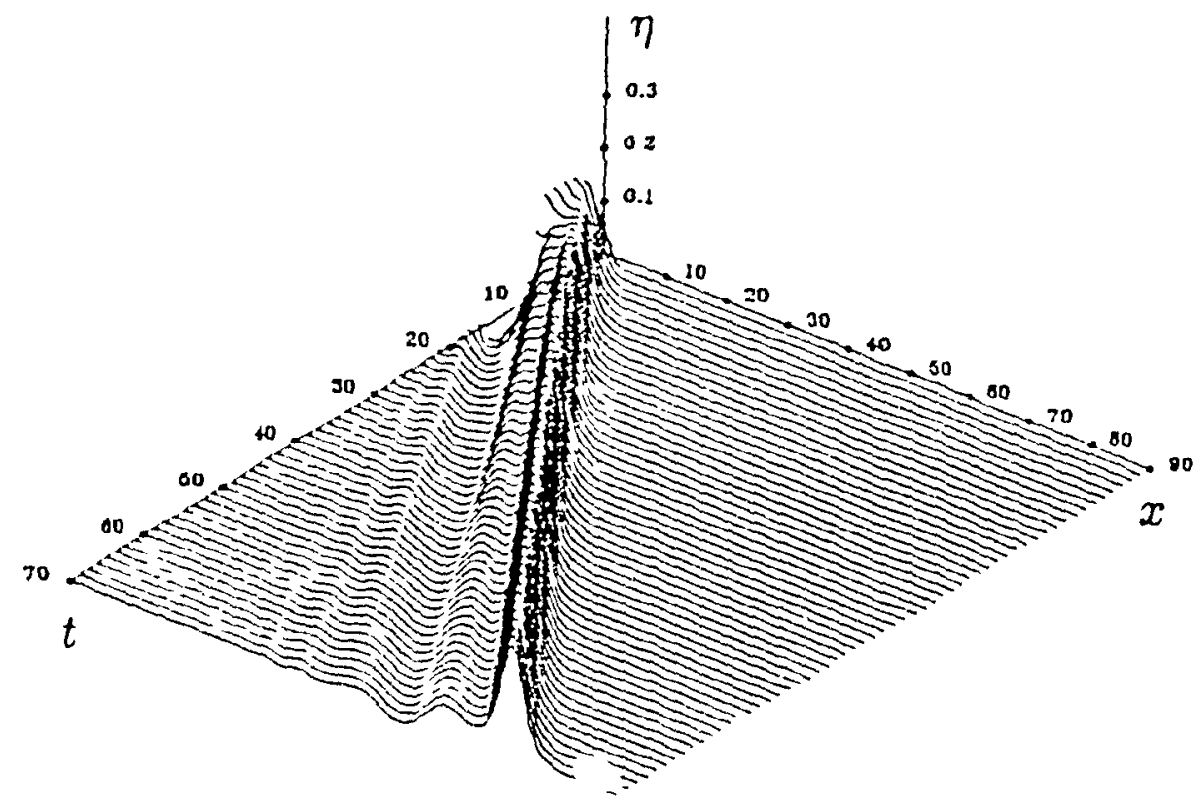

Figure 5.4a. Wave generated by a rotating plate.

In spute of the apparent smplicty of the wave generating device, the agreement herwern computed and observed wave herghts is not as good as in the previous cases. Ligute 5 th shows that the computed wave amplitude (dashed line) is always slightly larger than the experimental values (solid line) even in the initial generation stage. Therefore. unlike the case of the moving wall. the difference in amplitude cannot be smply attubuted to dissipatne effects in the wave channel.

It an also be observed that at gauge $1(x=10.3)$, the observed disintegration 


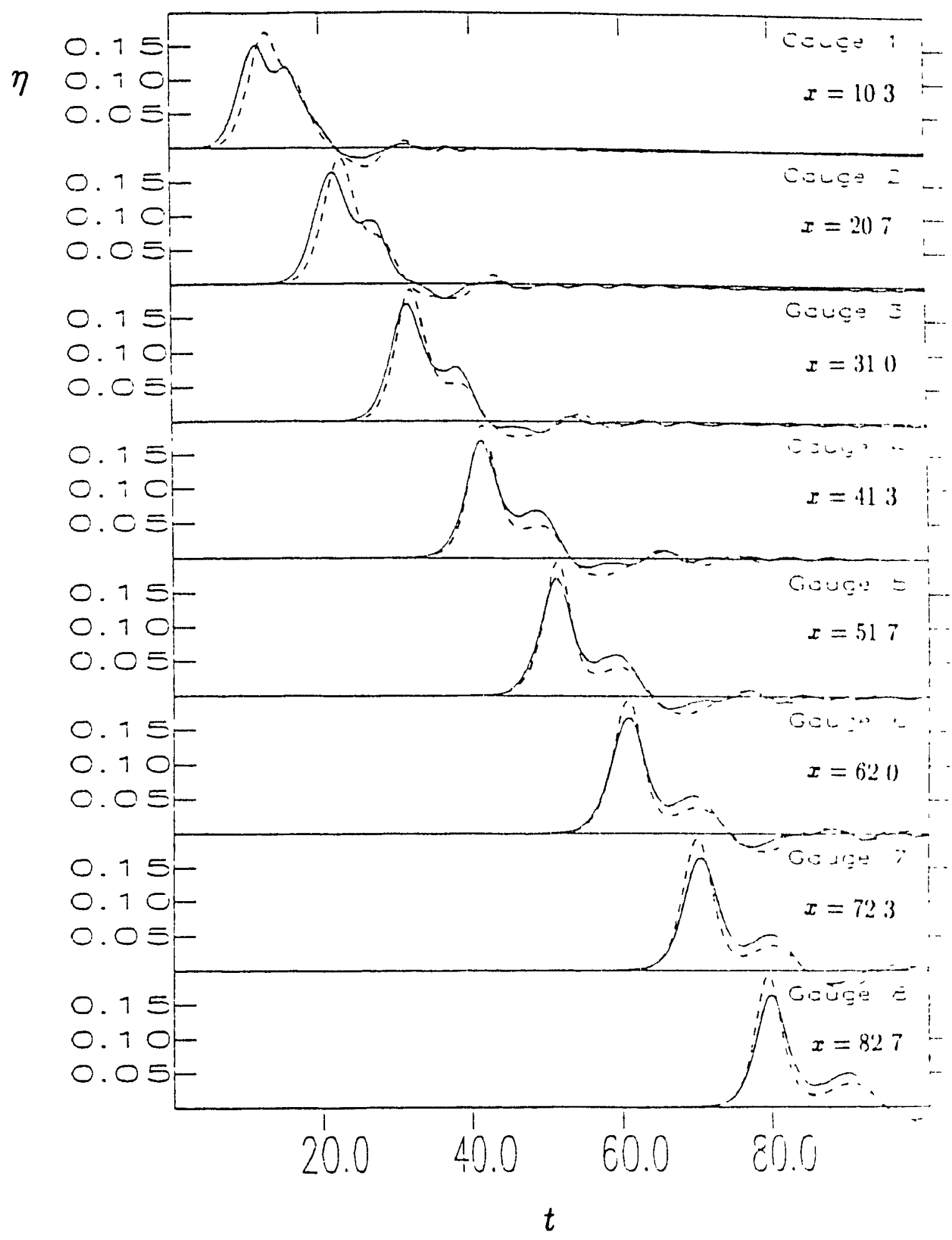

Figure 5.4b: Wave generated by a rotating plate. Length of the plate: $l=3: 3$ vertical pull velocity $V=0.1$ at $x=0$. duration of the bed motuon $t_{f}=x(1)$. $f_{m}$. slope $m_{\max }=0.24$. Experiment - computation - - 
of the intial disturbance into shorter humps is not clearly defined in the computed results

Ile alsove comments can also be applied to the results shown on figures D.3a and 1) 3h, of $A$ ppendix D

Two reasoms may explain these small discrepancies. First, it is possible that the Impoustion of a constant and impulsive pull velocity at the end of the plate imsen slight mstabulitues in the numerical model. Secondly, it appears that in all the ummlated rotating plate problems, the final slope was rather low $(\approx 0.2)$. Thus the whitun of llup plate manly moluces a vertical motion component to the water layer "lwe. In In thene comditions. the depth-averaged relocity assumption made in the

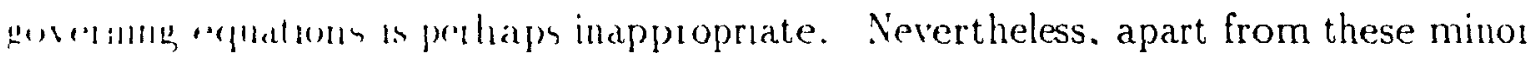

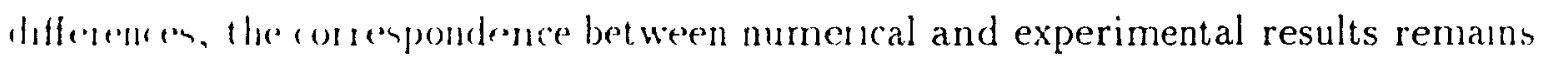
funte salufartory As seen in figure 5.4h, the wiodel gives a good representation of lenth the loathing and thathng wates

\subsection{Moving wedge}

lhe lant mung bed poblem that was considered involves the generation of a wave hi the lateral displacement of an inclined wall intersecting the water surface.

In mentundealier, the treatment of this problem is complicated by the presence of a moning waterhne The program WEDGE was used to compute the resulting "awe putilen The F('I' algonthm was again used in this case. Without FCT, the computed colutions suffered from severe instablities.

Figumen 5 . 3 and $55 \mathrm{~b}$ give the evolution of a wave created by the motion of a wedge of slope $m=0.268$ which moves with a sinusoidal velocity from $x=0$ to $r=16$ in a tme of $13.1 \%$. In figure $5.5 \mathrm{~b}$, the instantaneous position of the moving Watcrline is induated by a curve in the $x$-t plane.

It appears that, during the initial rise of water, the $x$-coordinate of the waterline lcmanns at $x=0$. As soon as the wave leaves the origin, the water depth comes 
back to its undisturbed level as indicated by the position of the waterline which star constant at $x \approx 1.67$.

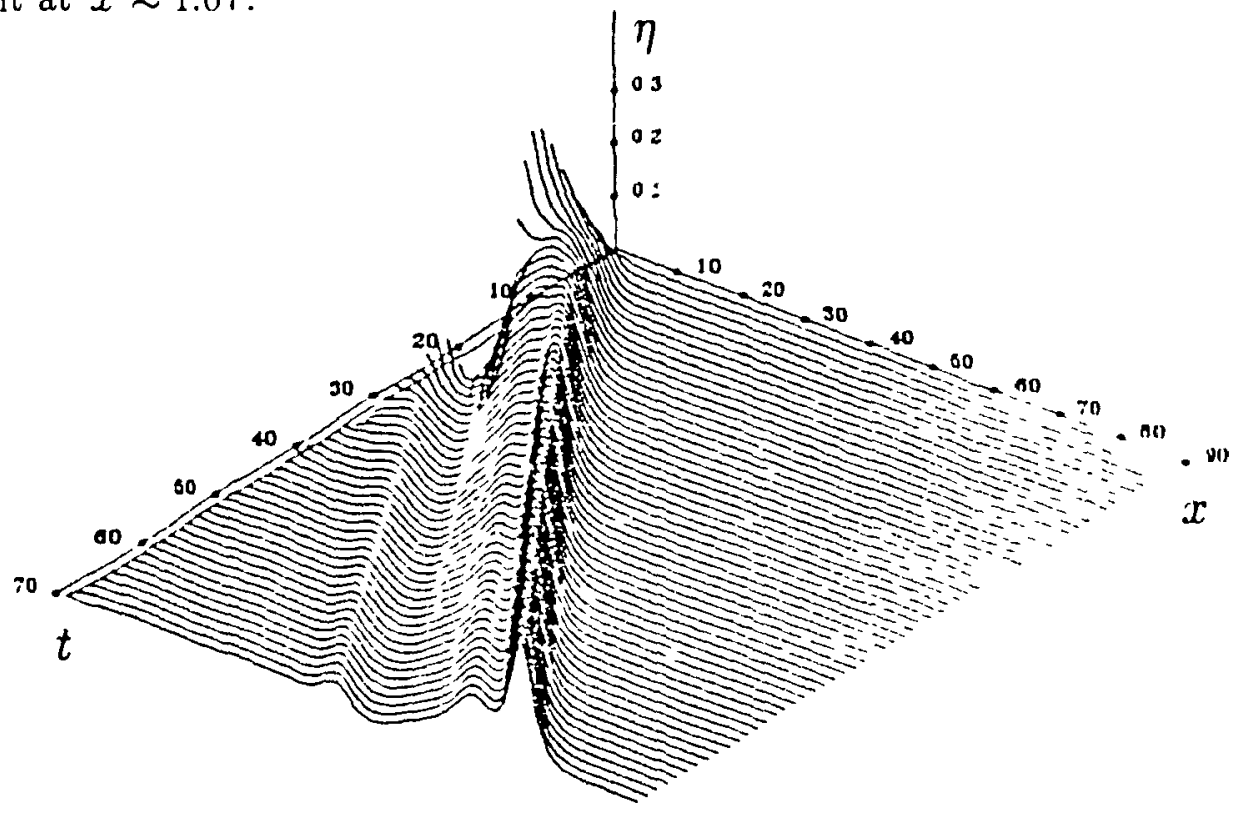

Figure 55a: Wave generated by a moving wedge

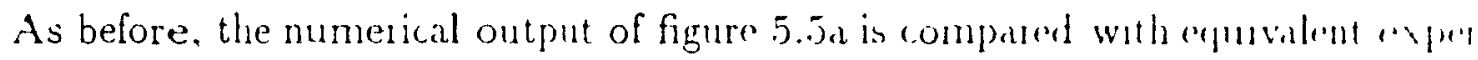
imental results in figure $55 \mathrm{~b}$. The agreement is excellent The computed amplitume and wavelength of both the leading and trallng waves match almost penferty the experimental values

The effect of the bed slope discontinusty on the evaluation of the derivatuve $h_{1}$. With studied by replacing that discontmuity by a smooth transition as given by efuatun (4.3). No improvement was observed in the computed solution.

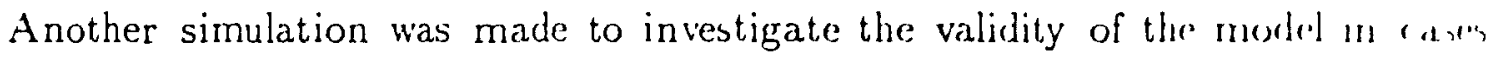
1nvolving a steeper wedge slope. Figure D.4 shows calculated and morasmerl wave heights for a moving wedge of slope $m=1$. As in the previous case, the comput,atum, closely match the experiment.

The above results show that the Lagranglan treatment, of the moving wat erlume gives unexpectedly good results in spite of its relatuve smplucity Furthermon.. Hary confirm the validity of the proposed model for the accurate descriputon of wares generated by moving boundaries 


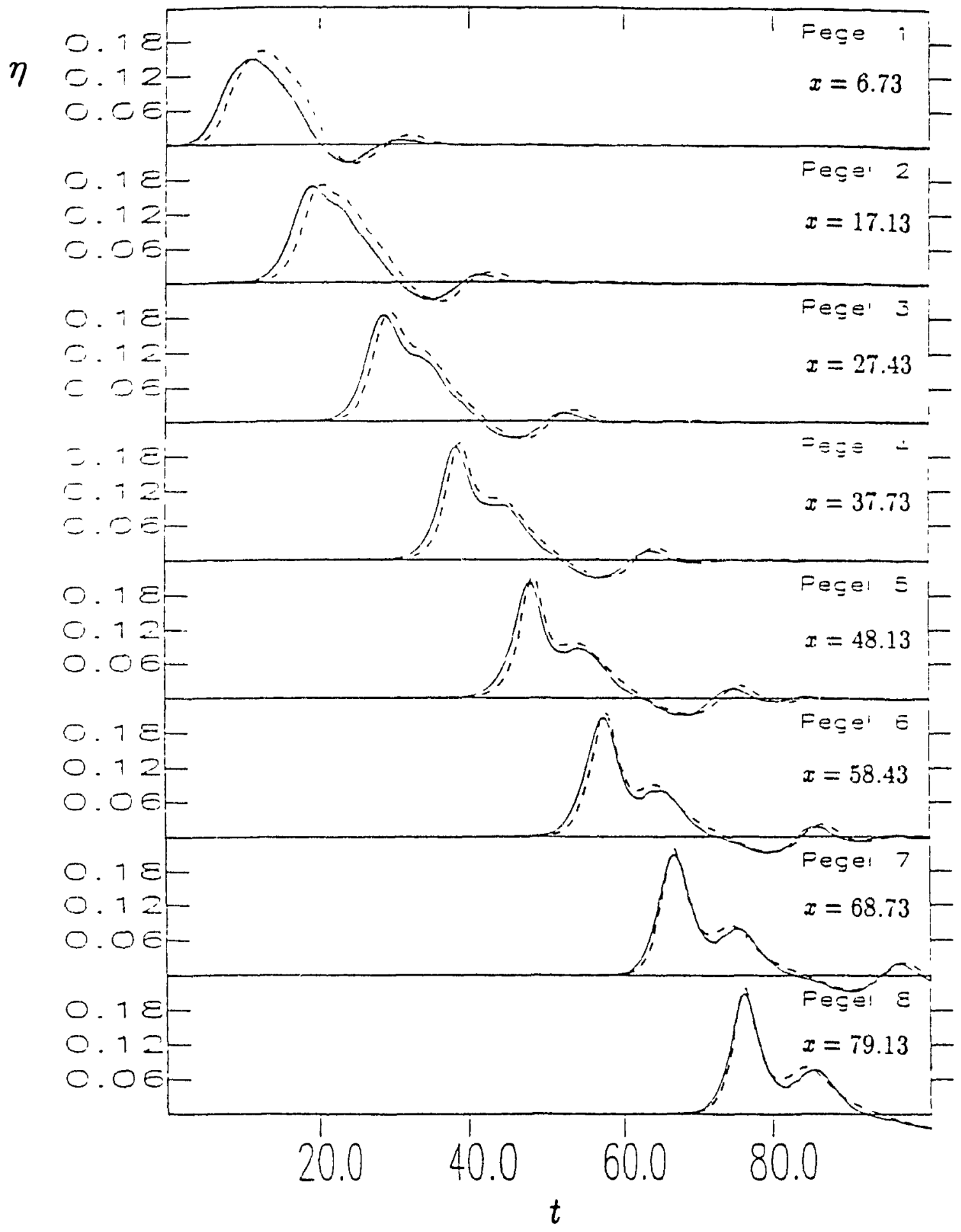

Figure 5 it) Wave generated by a moving wedge. Slope $m=0.268$; maximum horkontal velocity of the wedge $v^{*}=0.2$; total displacement $d=1.67$; duration of the bed motion $t_{f}=13.12$. Experiment - computation $\cdots$. 


\section{CONCLUSIONS}

Natural phenomena involving the generation and propagation of water waves aled oped by moving boundaries sometimes can constitute a serious threat to both coastal populations and man-made structures. Landshde induced water waves in lakes and tsunamis are well documerited examples of this type of problem.

In the preceding sections, a mathematical model was developed for the monlellum, of water waves generated by a moving bed As a starting point to more coulintu representations, only one-dimensional, frictionless problems were comsidered

The model relies on a set of depth-averaged continuty and momentum equalmun which constitute a generalization of the Boussinesq equations accounting for the un steady and nonuniform bed topography This theory is hmuted to wave prophgation in shallow water but it presents the advantage of combining in an apponumatr. balance-the two fundamental effects of nonlinearity (or amplitude dispersum) and dispersion (or frequency dispersion). Thus fature dimmates questoms aboul 1 ha. range of applicability of the theory and in that respect constitute a major advintane over small-amplitude and finite-amplitude (Ary) thoores.

A simple numerical solution to the governing equatuons was obtaned by mentur of finte difference approximations In ordes to merease the acurary, the compulat

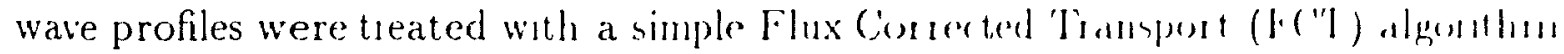
That scheme was not only useful in upgradung the accuracy of the solutums. It al th" lead to accurate solutions in cases where a stable solution conld uot reven lae oblanded without FCT.

The numerical results presented in 8.5 showed an excellent agreerment, with ar responding expermental results. The ampliturle, wave length and velosity of holh the leading wave and disperswe trailing waves were veny well preducted by the morle. (except perhaps in the case of the rotaing plate for which the shallow-watrip apde. imation $u(x, y, t) \simeq \bar{u}(x, t)$ was maybe mippropriate).

Therefore, we conclude that the nonhnear. disprersive, shallow wat , wave malh 
ematiral model presented herein constitutes a very good representation for the generation and propagation of waves developed by a moving bed.

Further studies could be directed towards the treatment of problems in two spatial limensions and on a more realistic representation of the moving bed in which the moving solid boundaries could be replaced by a sliding granular material. 


\section{REFERENCES}

BoRIs. J.P. 1981 A one-dimensional Lagrangian code for nearly incompressible then In Finate-dafference Technaques For lectorlzed Fluad Dynamuss Calculatum, (x) D.L. Book), $75-97$.

Boris, J.P. \& Book, D.L 1973 Flux-Corrected Transport. I. SHASTA, a thum transport algorithm that works. J. Comput. Phys. 11, 38-69.

Boris, J.P.,Book, D.L. \& HAN, K. 1975 Flux-Corected Transport. II (iemel alizations of the method J. Comput. Phys. 18, 248-283.

Bonis, J.P. \& Book, D.L. 1976 Flux-Corrected Transpott. III. Mmmmal 'tun FCT algorithms. J. Comput. Phys. 20, 397-431.

Carrier,G.F. \& Greenspan,H.P. 1958 Water waves of finite amplitude on a sloping beach. J. Flurd Mech. 4, 97-109.

DAS, M.M. \& WIEGEL, R.L. 1972 Waves generated by horizontal molion of a wall J. Waterw. Harbors Div. ASCE. 98, No. WW 1, 49-65

FRIEDRICis, K.O 19.48 On the derivation of the shallow water theory fapmidin 'The formation of breakers and bores' by J.J Stoker. Commun $1 / p l$. $1 /$ ull $1,81-85$.

HAMMACK, J L. 1973 A note on tsunamis: their generation and propagatwn 111 in ocean of uniform depth. J. Fluzd Mech. 60, 769-799.

Helfrich, K.R. \& Melville, W.K. 1986 On long nonlinear internal waves owe slope-shelf topography. J. Flund Mech. 167, 285-30s.

Hibberd, S. \& Percgrine, D H. 1979 Surf and run-up on a bear.h a mutrmu bore. J. Fluid Mech. 95, 323-345.

HUBER, A. 1982 Impulse waves in Siviss lahes as a result of rock avalinches and hronl. slides. Experimental results for the prediction of the characteristic. muntwin these waves. Quatorzième Congrès des Grands Barrages, Rio De Jan'mo, 19n: $45.5-475$.

Hunt, B. 1988 Water waves generated by distant lanclides. J. Hyd Res 26, 3317 32.

HWANG,L.S. \& DivokY, D. 1970 Tsunam generation J Ceophys lies 75 $6802-6817$.

KELLER, J.B. 1948 The solitary wave and persodic waves in shall,w watem Commun. Appl Math 1,323-333 
JoInson, R.S. 1972 Sorne numerical solutions of a variable-coefficient Korteweg-de Vries equation (with applications to solitary wave development on a shelf). $J$. Flund Mech. 54, 81-91.

KravZer, H.C. \& Keller, J.B. 1959 Water waves produced by explosions. J. Appl. Phys. 30, 398-407.

MADSEN, OS \& MEI, C.C. 1969 The transformation of a solitary wave over an uneven bottom. J. Fluzd Mech. 39, 781-791.

NoDA, E. 1970 Water waves generated by landslides. J. Waterw. Harbors Div. ASCE 96 , No. WW 4,835-855.

NoDA, E.K 1971 Water waves generated by a local surface disturbance. J. Geophys. Re's. 76, 7389-7400.

PIdichev, G. \& GueviK, B. 1983 Run-up of solitary waves. J. Fluid Mech. 135. $283-299$.

PLrigrine, D H 1967 Long waves on a beach. J. Fluzd Mech. 27, 815-827.

PlRkCRINE, D.H. 1972 Equations for water waves and the approximation behind them In Waves on Beaches and Resulting Sedıment Transport (ed. R.E. Meyer), Academic Press, 95-121.

Plntimine, D.H 1983 Breaking waves on beaches. Ann. Rev. Fluid Mech. 15, $149-1$ is.

Peyner, R. \& Taylon, T.D. 1983 Computational Methods for Fluid Flow. Springer-Verlag, 104-105.

PuINs, J.E. 1958 Characteristics of waves generated by a local disturbance. Trans. Am Geophys Unıon 39, S65-874.

RaNi:, D C. \& Butler, H L. 1976 Landslide generated water wave model. J. Hyd. Diw. ASCE. 102,No.HY'9, 1269-12S2.

Sabstili, P.C. 1983 On water waves produced by ground motions. J. Fluid Mech. $126,2 i-5 s$.

SANDER, J. 1988 Waves in lakes generated by rockslides. Internal report, Laboratory of Hydraulics, Hydrology and Glaciology (VAW) of the Swiss Federal Institute of Technology (ETH), Zürich.

Senbra-Sintos,F.G..Renouard,D.P. \& Temperville, A.M. 1987 Numerical and experimental study of the transformation of a solitary wave over a shelf or isolated obstacle. J. Flurd Mech. 176, 117-134. 
Slingerland, R.L. \& Vorght, B. 1979 Occurences, properties, and predic:1we models of landslide-generated water waves. In Rockshdes and dralanches, Ind 2 (ed. B. Voight), Elsevier, 31i-397.

SPIElvogel, L.Q. 1975 Single-wave run-up on sloping beaches. J. Fluad Mech. 74. 685-694.

Su, C.H. \& Gardner, C.S. 1969 Korteweg-de Vries equation and generalizations III. Derivation of the Korteweg-de Vries equation and Burgers equations I Math. Phys. 10, 536-539.

TUCK, E.O. \& HWANG, L S. 1972 Long wave generation on a sloping beath f Flund Mech. 51, 449-461.

URSELL, F. 195.3 The long-wave pardox in the theory of gravity waves Proc. ('amb) Phal. Soc. 49, 685-694.

VoIt, S.S. 1987 Tsunamis. Ann. Rev. Fluid Mech. 19, 217-2:36.

WIEGEL, R.L. ET aL 1970 Water waves generated by landslides in reservons, I Waterw. Harbors Div. ASCE 96, No.WW2, 307-333.

Wehausen,J.V. \& Laitonen,E.V. 1960 Surface waves. In Eincyclopedea of Physics, Vol.9 (ed. S. Flugge), Sprunger-Verlag, 617-619.

Whitha.M, G.B. 1974 Linear and Nonlinear Waves Wiley-Interscience, New-Youk

Wu, T.Y. 1981 Long waves in ocean and coastal waters. J. Fing. Merh. Dol AS' 'L' 107, No. EM3, 501-522.

ZaLeSAK, S.T. 1979 Fully multidimensional Flux-Corrected Transport algonithum for fluids. J. Comput. Phys 31, 335--362. 


\section{APPENDIX A}

\section{Validity of the shallow-water approximation}


As pointed out in $\S 2.1$, the depth-averaging of the $x$-momentum equation shows that the 'shallow-water' approximation-which merely consists in replacung $u(x, y, t)$ by $\bar{u}(x, t)$-is only valid if $H\left(\overline{u^{2}}-\bar{u}^{2}\right)$ is small compared to the remaining terms of the equation

$$
\frac{\partial}{\partial t}(H \bar{u})+\frac{\partial}{\partial x}\left[\left(H\left(\bar{u}^{2}+\bar{p}\right)+H\left(\overline{u^{2}}-\bar{u}^{2}\right)\right]=p_{b} \frac{\partial h}{\partial x} .\right.
$$

In other words, for $\overline{u^{2}}-\bar{u}^{2}$ to be negligible, we must show that it is of higherr orden than the expressions developed for $p_{b}$ and $\bar{p}$. From (2.22) and (2.23). we see thith the order of $p_{b}$ and $\bar{p}$ is determined by the order of the factors $\alpha, \beta$ and $\gamma$ (eymation $2.2(1)$ ) in terms of which they are expessed In the scaled equations (2 3:2) for $6^{*}, 3^{*}$, and $\gamma^{*}$, we saw that the higher order terms were $O\left(\epsilon^{2} \sigma^{2}\right)$. We therefore must delenmim under what conditions the difference $\overline{u^{2}}-\bar{u}^{2}$ becomes smaller than $O\left(c^{2} \sigma^{2}\right)$.

We first rewrite the approximation (2.12) for the vertical veloc ity as :

$$
v \simeq-\left[h_{t}+\bar{u} h_{x}+(y+h) \bar{u}_{x}\right]
$$

Combining (A2) with the irrotationality condition

$$
\frac{\partial u}{\partial y}=\frac{\partial v}{\partial x}
$$

we obtain

$$
\frac{\partial u}{\partial y}=-(y+h) \bar{u}_{x x}-D
$$

where

$$
D=h_{t x}+2 h_{J} \bar{u}_{x}+h_{L s} \bar{u}
$$

Integration of $(A 4)$ from $y$ to $\eta$ gives:

$$
u=u_{s}+\bar{u}_{x x}\left[\frac{H^{2}}{2}-\frac{(y+h)^{2}}{2}\right]+D(\eta-y)
$$

and from the definition (2.10) of the depth-averaged velocity, we have:

$$
\bar{u}=\frac{1}{H} \int_{-h}^{\eta} u d y=u_{s}+\bar{u}_{x x} \frac{H^{2}}{3}+D \frac{I I}{2} .
$$


Sublerating (AG) from $(A .5)$, we get

$$
u-\bar{u}=\bar{u}_{\perp 5}\left[\frac{I^{2}}{6}-\frac{(y+h)^{2}}{2}\right]+D\left[\frac{H}{2}-(y+h)\right] .
$$

Vister that $(\Lambda 7)$ can be reduced to

$$
u=\bar{u}-\left(y+\frac{h}{2}\right)\left[h_{t}+(h \bar{u})_{x}\right]_{x}-\left(\frac{y^{2}}{2}+\frac{h^{2}}{6}\right) \bar{u}_{x x}+O\left(\epsilon^{2} \sigma^{2}\right)
$$

as In equation (2.36). Squaring $u$ in equation (A7) gives

$$
\begin{aligned}
u^{2}=\bar{u}^{2} & +2 \bar{u}_{s x}\left[\frac{I^{2}}{6}-\frac{(y+h)^{2}}{2}\right]+2 \bar{u} D\left[\frac{H}{2}-(y+h)\right] \\
& +\bar{u}_{x x}^{2}\left[\frac{H^{1}}{36}-\frac{I^{2}(y+h)^{2}}{6}+\frac{(y+h)^{4}}{4}\right] \\
& +2 \bar{u}_{x x} D\left[\frac{H^{3}}{12}-\frac{H(y+h)^{2}}{4}-\frac{H^{2}(y+h)}{6}+\frac{(y+h)^{3}}{2}\right] \\
& +D^{2}\left[\frac{I^{2}}{4}(y+h)+(y+h)^{2}\right]
\end{aligned}
$$

and applyng the defintion (2) 17$)$ of $\overline{u^{2}}$ to $(A S)$, we finally obtain

$$
\bar{u}^{2}-\bar{u}^{2}=\bar{u}_{x x}^{2} \frac{H^{4}}{45}+\bar{u}_{x x} D \frac{H^{3}}{12}+D^{2} \frac{H^{2}}{12} \text {. }
$$

Fin wales over a flat horrontal bed, $D=0$ and (A9) reduces to

$$
\bar{u}^{2}-\bar{u}^{2}=\bar{u}_{r x}^{2} \frac{H^{4}}{45}
$$

an lowed by Sin \& Gadner (1969) The difference $\overline{u^{2}}-\bar{u}^{2}$ as given by $(A 10)$ is () $\left(c^{2} \sigma^{\prime}\right)$ and is always negligible selative to the $O\left(\epsilon^{2} \sigma^{2}\right)$ pressure terms. Therefore. "we comluke that there is no restriction on the applicability of the shallow-" ater" anmmptun $u(r, !, t) \simeq \pi(r, t)$ lo1 long waves over a flat bed.

Humere, if the bed $k m$ motion. $D$ is non-zero and depends on the slope and wherts of the bed 1 - nummg the orders of magnitude of the slope $\frac{\partial h}{\partial x}$ and velocity " to be mhknwms, we an use the scalng factors defined by (2.27) to write

$$
D^{*}=-\left[\sigma \nu^{\prime}\left(h_{t}\right)_{x^{*}}^{*}+2 \iota \sigma \mu\left(h_{x}\right)^{*} \bar{u}_{x}^{*}+\epsilon \sigma \mu\left(h_{x}\right)_{x}^{*} \cdot \bar{u}^{*}\right]
$$


where it is assumed that $\mu$ and $\nu$ respectively represent the orden of magnitule of the slope and velocity of the bed, i. e.

$$
\begin{aligned}
& \frac{\partial h}{\partial x}=O(\mu), \\
& \frac{\partial h}{\partial t}=O\left(\iota^{\prime}\right)
\end{aligned}
$$

As mentionned before, the first term on the right hand skde of (Ag) wall alwan be smaller than $O\left(\epsilon^{2} \sigma^{2}\right)$. Therefore, for the difference $\overline{u^{2}}-\bar{u}^{2}$ to be neglighthe, we ment

$$
\bar{u}_{\alpha 2} D \frac{H^{3}}{12}<O\left(c^{2} \sigma^{2}\right)
$$

and

$$
D^{2} \frac{H^{2}}{12}<O\left(c^{2} \sigma^{2}\right)
$$

Using the scales defined in (227) and (A11), we see that the comdllam $(112)$, mil (A13) tespectively imply.

$$
\left.\begin{array}{c}
\left(c \sigma^{3}\right) \mu \\
\left(\sigma^{2} \sigma^{3}\right) \mu
\end{array}\right\}<c^{2} \sigma^{2}
$$

and

$$
\left.\begin{array}{r}
\sigma^{2} \nu^{2} \\
\left(\epsilon^{2} \sigma^{2}\right) \mu^{2} \\
\left(c \sigma^{2}\right) \mu l
\end{array}\right\}<e^{2} \sigma^{2}
$$

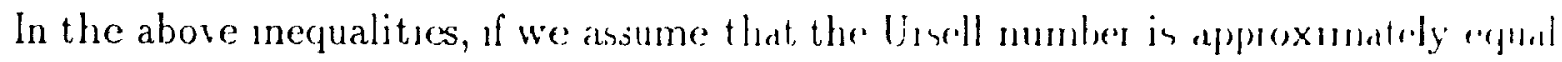

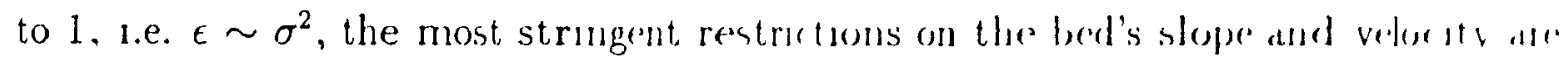
found to be:

$$
\mu<1 \text { and } \nu<1
$$

The slope of the bed must then be

$$
\frac{\partial h}{\partial x}<O(1)
$$

and its velocity

$$
\frac{\partial h}{\partial t}<O(r)
$$


Wr comelude that for long waves generated by a moving bed, the approximation $u(r, y, l) \simeq \bar{u}(x, \imath)$ is only valud if the conditions (A14) and (A1,5) are satisfied. Nev"Itheress, the very wide range of realistic bed motions that can be described within $H_{1} \cdot h_{m}$ ts of $(A 14)$ and $(A 1.5)$ justifies the elimination of the term $H\left(\overline{u^{2}}-\bar{u}^{2}\right)$ from the gevernugh momentum equation (224).

Finally, we chere ve that these conditions are satisfied by the scales

$$
\begin{aligned}
& \frac{\partial h}{\partial r}=O(\sigma) \\
& \frac{\partial h}{\partial t}=O(\epsilon \sigma)
\end{aligned}
$$

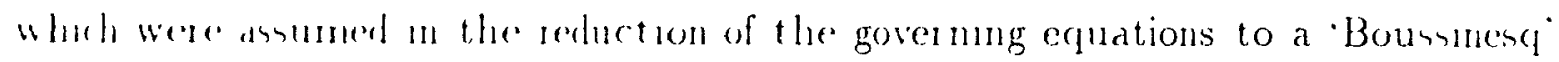
onder in 322 With $(A \mid 6)$ and $(A 1 T)$, the term $D$ given by $(A 11)$ becomes $O\left(6 \sigma^{2}\right)$ and hom (A!) we therefore obsan

$$
\overline{u^{2}}-\bar{u}^{2}=O\left(c^{2} \sigma^{1}\right)
$$

an 11 Win $(1981)$ 
APPENDIX B

\section{Derivation of the governing equations using an expansion method}


'The momentum equation (2.34) of 'Boussinesq' order was obtained by scaling undelumnating the higher-order terms from the 'complete' depth-averaged equation $(224)$

An alternative procedure can be used to derive equation (.2.34) directly from the fundamental equations of motion The method basically consists in expanding the dependent variables of the problem in terms of the small parameters $\sigma$ and $\epsilon$ defined III 322 These expansions are then substituted in the equations of motion and the boundaty conditions, and coefficients of like powers of $\sigma$ and $\epsilon$ are equated. The ongmal poblem is then effectively split mto simpler sub-problems each associated with a yeresfir order of magnitude indicated by the power of the small factors $\sigma$ and - The solution to the exact hydrodynamic problem can then be approximated by the sum of the solutions to these sub-problems, from order zero up to the required order

The above proced ae, which is based on the introduction of one or more small patameter(s) un wew of amplifyng the solution of a complex problem. is called a perturbution method The formal application of perturbation methods in the theor! of hiallow-water waves has been described by Friedrichs (1948) and Keller (1948) Wie here evend the procedure described by Petegrine (1967) to account for the effects of moving bed The following denvation is limited to two spatial dimensions, although the treatment of thee dimemsons does not present further difficulties

Wie reall from $\xi 2.1$ the fundamental equations of motion in dimensionless form $(\because 1)(\because 1)$

$$
\begin{gathered}
\frac{\partial u}{\partial x}+\frac{\partial v}{\partial y}=0, \\
\frac{\partial \jmath_{u}}{\partial t}+u \frac{\partial u}{\partial x}+v \frac{\partial u}{\partial y}=-\frac{\partial p}{\partial x}, \\
\frac{\partial v}{\partial t}+u \frac{\partial v^{\prime}}{\partial x}+v \frac{\partial v}{\partial y}=-\frac{\partial p}{\partial y}-1, \\
\frac{\partial u}{\partial y}=\frac{\partial v}{\partial x}
\end{gathered}
$$


and write the boundary conditions $(2.5)-(2 \pi)$ as

$$
\begin{gathered}
v=\frac{\partial \zeta}{\partial t}+u \frac{\partial \zeta}{\partial x} \text { at } y=\zeta(x, t), \\
v=-\frac{\partial h}{\partial t}-u \frac{\partial h}{\partial x} \text { at } y=-h(x, t), \\
p=0 \text { at } y=\zeta(x, t)
\end{gathered}
$$

where the wave amplotude is now denoped by 5 (as opponed to $\eta$ m gi:). I'ung the kinematic boundary conditions (B5) and (B6), we mteglate the contimuty equallun (B1) over the depth to obtain

$$
\frac{\partial \zeta}{\partial t}+\frac{\partial \partial}{\partial r}=-\frac{\partial h}{\partial t}
$$

where

$$
Q=\int_{-h}^{n} u d y
$$

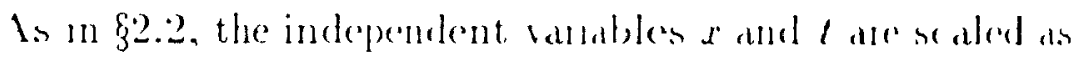

$$
(r, t)=\frac{1}{\sigma}\left(x_{1}, t_{1}\right)
$$

where the scaled variables are here denoted by the subscapt I (an astenuh wat used in $\$ 2.2)$. We also assume that

$$
\frac{\partial h}{\partial t}=\left(\frac{\partial h}{\partial t}\right)_{1}+e\left(\frac{\partial h}{\partial t}\right)_{1}+c^{2}\left(\frac{\partial h}{\partial t}\right)_{2}
$$

The dependent variables $\zeta, u, p$ and $Q$ ancerpanded as.

$$
\begin{aligned}
\zeta & =\zeta_{0}+c \zeta_{1}+c^{2} \zeta_{2}+ \\
u & =u_{0}+\epsilon u_{1}+\epsilon^{2} u_{2}+\cdot \\
p & =p_{0}+\epsilon p_{1}+c^{2} p_{2}+\cdots \\
Q & =Q_{0}+\epsilon Q_{1}+\epsilon^{2} Q_{2}+\cdots
\end{aligned}
$$

and for (B1) to be consistent, we write:

$$
v=\sigma\left(v_{0}+c v_{1}+\cdots\right)
$$


At order gerso(se $\sigma=t=0)$. the solution will be taken to be still water, so that

$$
p_{0}=-y
$$

which implese that $\zeta_{0}, u_{0}, v_{0},\left(\frac{\partial h}{\partial t}\right)_{0}$ and $Q_{0}$ are all equal to zero. The still water solution was inplicitely included in the derivation used in $\S 2.2$ when we wrote $\bar{u}=$ ()$, \eta=O(c)$ and $h_{t}=O(\epsilon \sigma)$ Note that if (B11) and the expansions (B12) are whully sulstituted in the equations (B1)-(B9) the zcio-order equations turn out to be

$$
\begin{gathered}
\frac{\partial \zeta_{0}}{\partial t_{1}}+\frac{\partial}{\partial x_{1}}\left[\left(h+\zeta_{0}\right) u_{0}\right]=-\left(\frac{\partial h}{\partial t}\right)_{0}, \\
\frac{\partial u_{0}}{\partial t_{1}}+u_{0} \frac{\partial u_{0}}{\partial x_{1}}+\frac{\partial \zeta_{0}}{\partial x_{1}}=0
\end{gathered}
$$

whuch are the well-hmown Any (finite-amplitude, shallow-water) equations.

The first onder soluteon melerived as followe At $O(t)=O\left(\sigma^{2}\right)$, the urotationality comditum (B3t) becomen

$$
\frac{\partial u_{1}}{\partial y}=0
$$

$\operatorname{since} r_{0}=0$. Hence,

$$
u_{1}=u_{1}\left(r_{1}, t_{1}\right)
$$

and thereforn, from (B9):

$$
Q_{1}=h u_{1}
$$

The ()(e) y-momentume equation is

$$
\frac{\partial p_{1}}{\partial y}=0
$$

and thom the stress fee boundary condition (BT)

$$
p_{0}+\epsilon p_{1}=0 \text { at } y=\zeta_{0}+\epsilon \zeta_{1} .
$$

we obtum

$$
p_{1}=\zeta_{1}
$$


Substitution of $(B 1+f)-(B 16)$ in $(B 2)$ and (BS) gnes the following fit st-onder epuatume

$$
\begin{gathered}
\frac{\partial \zeta_{1}}{\partial t_{1}}+\frac{\partial\left(h u_{1}\right)}{\partial x_{1}}=-\left(\frac{\partial h}{\partial t}\right)_{1} \\
\frac{\partial u_{1}}{\partial t_{1}}+\frac{\partial \zeta_{1}}{\partial x_{1}}=0
\end{gathered}
$$

which are the linearized long-wave cquations. The first-order vertical vedouly ampu

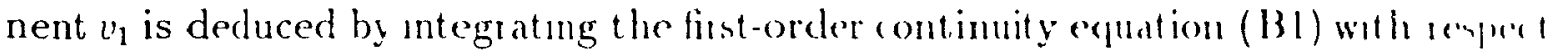
to $y$ :

$$
u_{1}=-y \frac{\partial u_{1}}{\partial x_{1}}+V_{1}\left(x_{1}, t_{1}\right)
$$

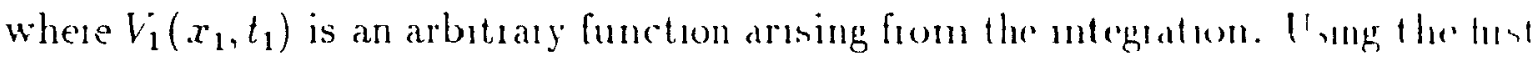
order kinematic boundary condition at the bed

$$
v_{1}=-\left(\frac{\partial h}{\partial t}\right)_{1}-u_{1} \frac{\partial h}{\partial x_{1}} \text { at } y=-h,
$$

we derfuce:

$$
I_{1}=-\left(\frac{\partial h}{\partial t}\right)_{1}-u_{1} \frac{\partial h}{\partial l_{1}}-h \frac{\partial u_{1}}{\partial x_{1}}
$$

and can then write

$$
v_{1}=-\left(\frac{\partial h}{\partial t}\right)_{1}-\frac{\partial\left(h u_{1}\right)}{\partial x_{1}}-y \frac{\partial \|_{1}}{\partial x_{1}}
$$

The above proc dure is repeated in onder to derwe the seond-onder equalum At $O\left(\epsilon^{2}\right)=O\left(c \sigma^{2}\right)$, irrotationality (B.l) give's

$$
\frac{\partial u_{2}}{\partial y}=\frac{\partial u_{1}}{\partial c_{1}}
$$

in which ( $\mathrm{B} 19)$ can be substituted to olstan

$$
\frac{\partial u_{2}}{\partial y}=-\frac{\partial}{\partial x_{1}}\left(\frac{\partial h}{\partial t}\right)_{1}-\frac{\partial^{2}\left(h u_{1}\right)}{\partial x_{1}^{2}}-y \frac{\partial^{2} u_{1}}{\partial x_{1}^{2}}
$$

Integration with respect to y leads to

$$
u_{2}=U_{2}\left(x_{1}, h_{1}\right)-y \frac{\partial}{\partial x_{1}}\left(\frac{\partial h_{h}}{\partial t}\right)_{1}-y \frac{\partial \partial^{2}\left(h u_{1}\right)}{\partial x_{1}^{2}}-\frac{y^{2}}{2} \frac{\partial^{2} u_{1}}{\partial x_{1}^{2}}
$$


whers $\left(I_{2}\left(r_{1}, t_{1}\right)\right.$ is an arbitrary function of integration. The $O\left(\epsilon^{2}\right) y$-momentum (q)iation

$$
\frac{\partial p_{2}}{\partial y}=-\frac{\partial v_{1}}{\partial t_{1}}
$$

now moludes the effect of the local vertical acceleration of the fluid. Hence, from (B19), we have.

$$
\frac{\partial p_{2}}{\partial y_{y}}=\frac{\partial}{\partial t_{1}}\left(\frac{\partial h}{\partial t}\right)_{1}+\frac{\partial^{2}\left(h u_{1}\right)}{\partial t_{1} \partial x_{1}}+y \frac{\partial^{2} u_{1}}{\partial t_{1} \partial x_{1}}
$$

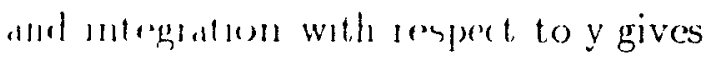

$$
p_{2}=\eta_{2}+y \frac{\partial}{\partial t_{1}}\left(\frac{\partial h}{\partial t}\right)_{1}+y \frac{\partial^{2}\left(h u_{1}\right)}{\partial t_{1} \partial x_{1}}+\frac{y^{2}}{2} \frac{\partial^{2} u_{1}}{\partial t_{1} \partial x_{1}}
$$

In whuth the besundary condation (B BT)

$$
p=p_{0}+\epsilon p_{1}+\epsilon^{2} p_{2}=0 \text { at } y=\zeta_{0}+\epsilon \zeta_{1}+\epsilon^{2} \zeta_{2}
$$

hou been used to determune the abbitrary function arising from the integration From Here defintuen (BS) of $Q$, we also have

$$
\epsilon^{2} Q_{2}=\int_{0}^{c \zeta_{1}} \epsilon u_{1} d y+\int_{-h}^{0} \epsilon^{2} u_{2} d y
$$

what. fiom $(13: 20)$

$$
Q_{2}=\zeta_{1} u_{1}+h u_{2}+\frac{h^{2}}{2} \frac{\partial}{\partial x_{1}}\left[\left(\frac{\partial h}{\partial t}\right)_{1}+\frac{\partial\left(h u_{1}\right)}{\partial x_{1}}\right]-\frac{h^{3}}{6} \frac{\partial^{2} u_{1}}{\partial x_{1}^{2}}
$$

Subutitutum of $(B 20)(B 2 \cdot 2), n(B 2)$ and $(B S)$ gives the following second order equa(1011)

$$
\begin{aligned}
& \frac{\partial \zeta_{2}}{\partial t_{1}}+\frac{\partial Q_{2}}{\partial x_{1}}=-\left(\frac{\partial h}{\partial t}\right)_{2}, \\
& \frac{\partial U_{2}}{\partial t_{1}}+u_{1} \frac{\partial u_{1}}{\partial x_{1}}+\frac{\partial \zeta_{2}}{\partial x_{1}}=0 .
\end{aligned}
$$

As pounted out by Peregine (1967), the fir t-order linearized long-wave equations $(B 1 i)$ and $(B 1 S)$ are only valid for small values of $t_{1}$. After only a short propagation Imine, the second order effects of amplitude and frequency dispersion included in (B23) and (1321) effectwely become first-order effects. The final governing equations must 
therefore include "fist-order variables incorporating second-order terms". Fon the wave amplitude, we let

$$
\eta=\dot{c}_{1}+c^{2} \dot{\zeta}_{2}
$$

For the horizontal flud velocity, we introduce the depth averaged velority which we define as

$$
\begin{aligned}
\bar{u} & =\frac{1}{h+\eta}\left(\epsilon Q_{1}+\epsilon^{2}\left(Q_{2}\right)\right. \\
& =\epsilon u_{1}+\epsilon^{2}\left\{U_{2}+\frac{h}{2} \frac{\partial}{\partial x_{1}}\left[\left(\frac{\partial h}{\partial t}\right)_{1}+\frac{\partial\left(h u_{1}\right)}{\partial x_{1}}\right]-\frac{h^{2} \partial^{2} u_{1}}{\partial} \frac{\partial x_{1}^{2}}{}\right\} .
\end{aligned}
$$

Note that the choice of $\bar{u}$ to morporate second-orden effecto is not the only altentin tive. The final govenning equations can also be formulated on temms of the veluenty at the bed (Madsen \& Mei 1969) or by means of the velocity at $y=0$.

$$
u(r, 0, t)=c u_{1}+c^{2} U_{2} .
$$

Combining (B17) and (BLS) with (B2:3) (B26), and going back to the olghtol variables, we finally obtain

$$
\begin{gathered}
\frac{\partial \eta}{\partial t}+\frac{\partial}{\partial x}[(h+\eta) \bar{u}]=-\frac{\partial h}{\partial t} \\
\frac{\partial \bar{u}}{\partial t}+\bar{u} \frac{\partial \bar{u}}{\partial x}+\frac{\partial \eta}{\partial x}=\frac{h}{2} \frac{\partial^{2}}{\partial t \partial x}\left[\frac{\partial h}{\partial t}+\frac{\partial(h \bar{u})}{\partial x}\right]-\frac{h^{2}}{6} \frac{\partial^{3} \bar{u}}{\partial t \partial \cdot x^{2}} .
\end{gathered}
$$

Equations (B27) and (B28) are respectively identical to equations (29) and (231) derived in $\S 2$. The depth-averaged continuty equation (B27) is exact while the dephlsaveraged momentum equation (B2s) has an error tem of order $\left(0\left(1^{2} \sigma^{3}\right)\right.$ 
APPENDIX C

Computer programs 


\section{Program WALL}

\section{Purpose of the program}

Modelling of nonlinear, dispersive, shallow-water waves generated in a channel by a moving vertacal wall.

\section{Definition of the constants}

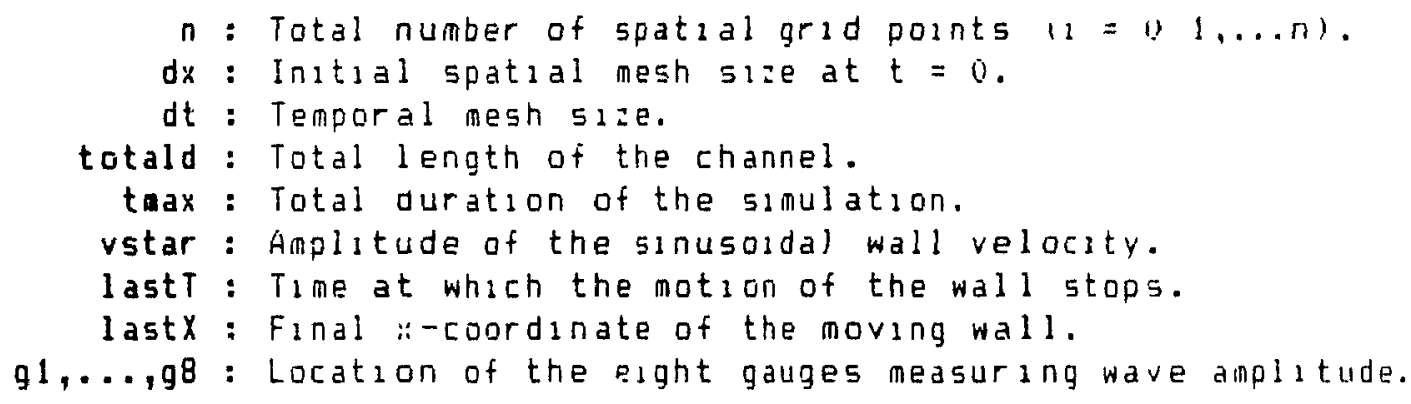

\section{Definition of the global variables}

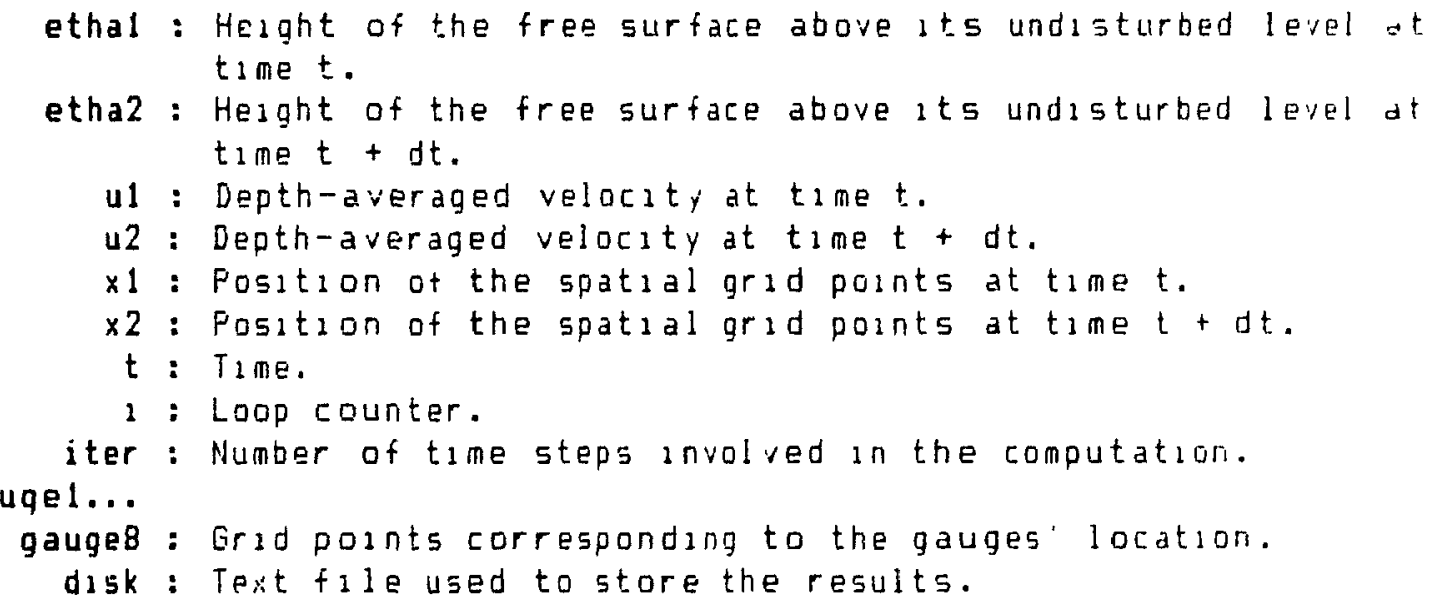




\section{Program listing}

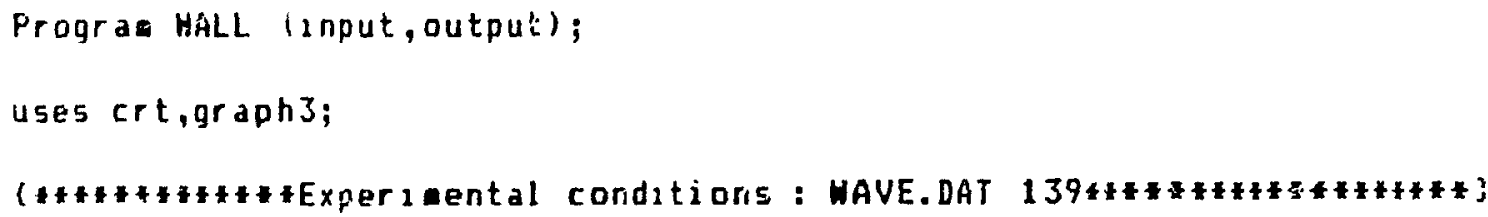

CONST

$$
\begin{aligned}
n & =500 ; \\
d x & =0.25 ; \\
d t & =0.25 ; \\
\text { totald } & =125 ; \\
\text { tmax } & =125 ; \\
v 5 t a t & =0.2 ; \\
\text { last } & =13.12 ; \\
\text { last } & =1.67 ; \\
91 & =3.7 ; \\
92 & =14.1 ; \\
93 & =24.4 ; \\
94 & =34.7 ; \\
95 & =45.1 ; \\
96 & =55.4 ; \\
97 & =65.7 ; \\
98 & =76.1 ;
\end{aligned}
$$

TYPE

vector = array $[0 \ldots n]$ of real;

VAR

ethal,ethaz,ul,u2 : vector;

$x !, \times 2$ : vactor:

$t$ : reai;

1, lter : integer;

gaugel,gauge2,gauge3,gauge4: integer;

gauges,gaugeb,gauge7, gauges : 2 nteger;

disk : text;

Function UPISTON(t:realiareal;

begin

if $t<=$ last

THEN

vpiston: = vstar $\sin \left(p_{1}+t / 1\right.$ ast $\left.T\right)$

ELSE

end;

vpsiston: $=0.0$ 
Function ACCPISTON(t:real):real;

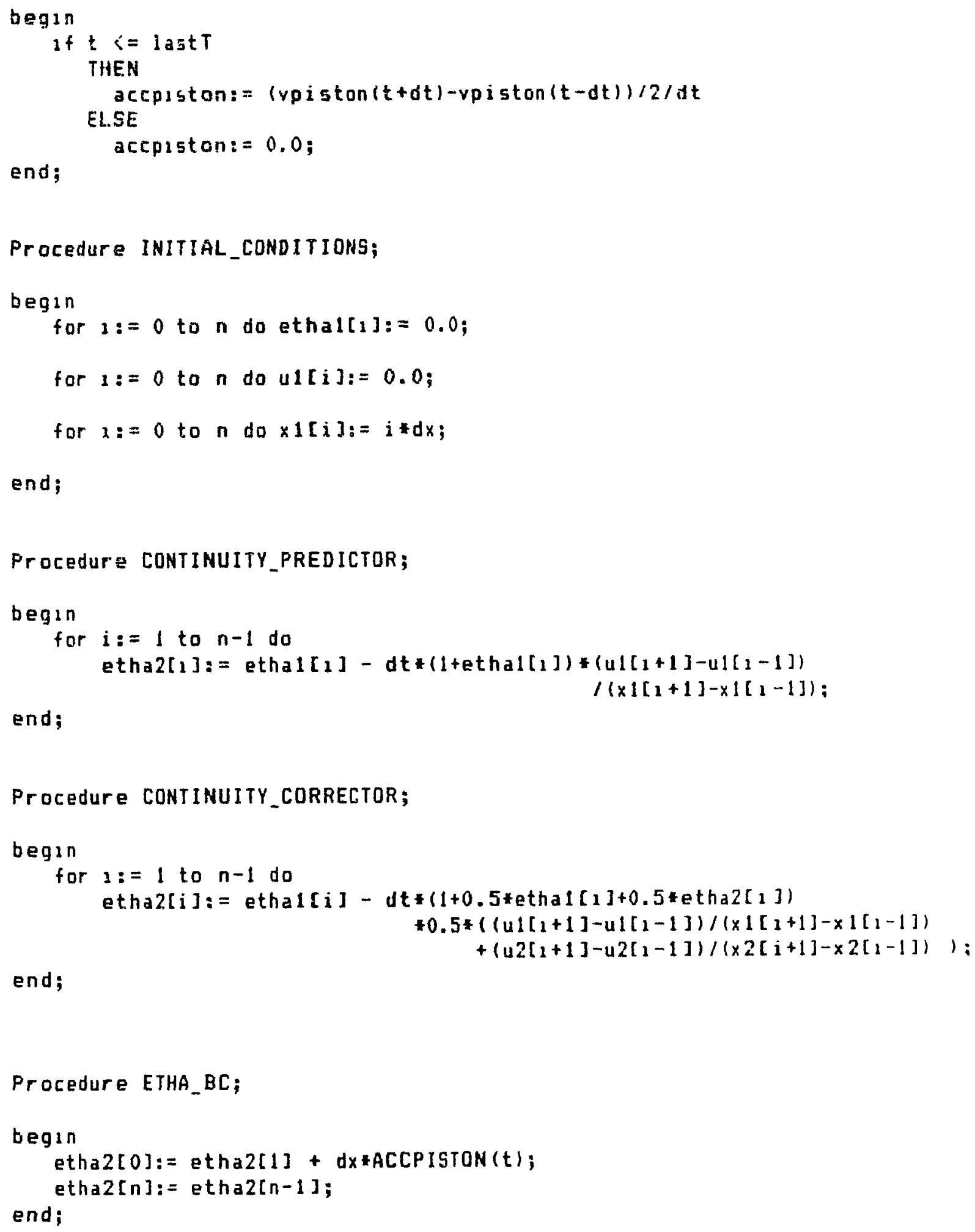




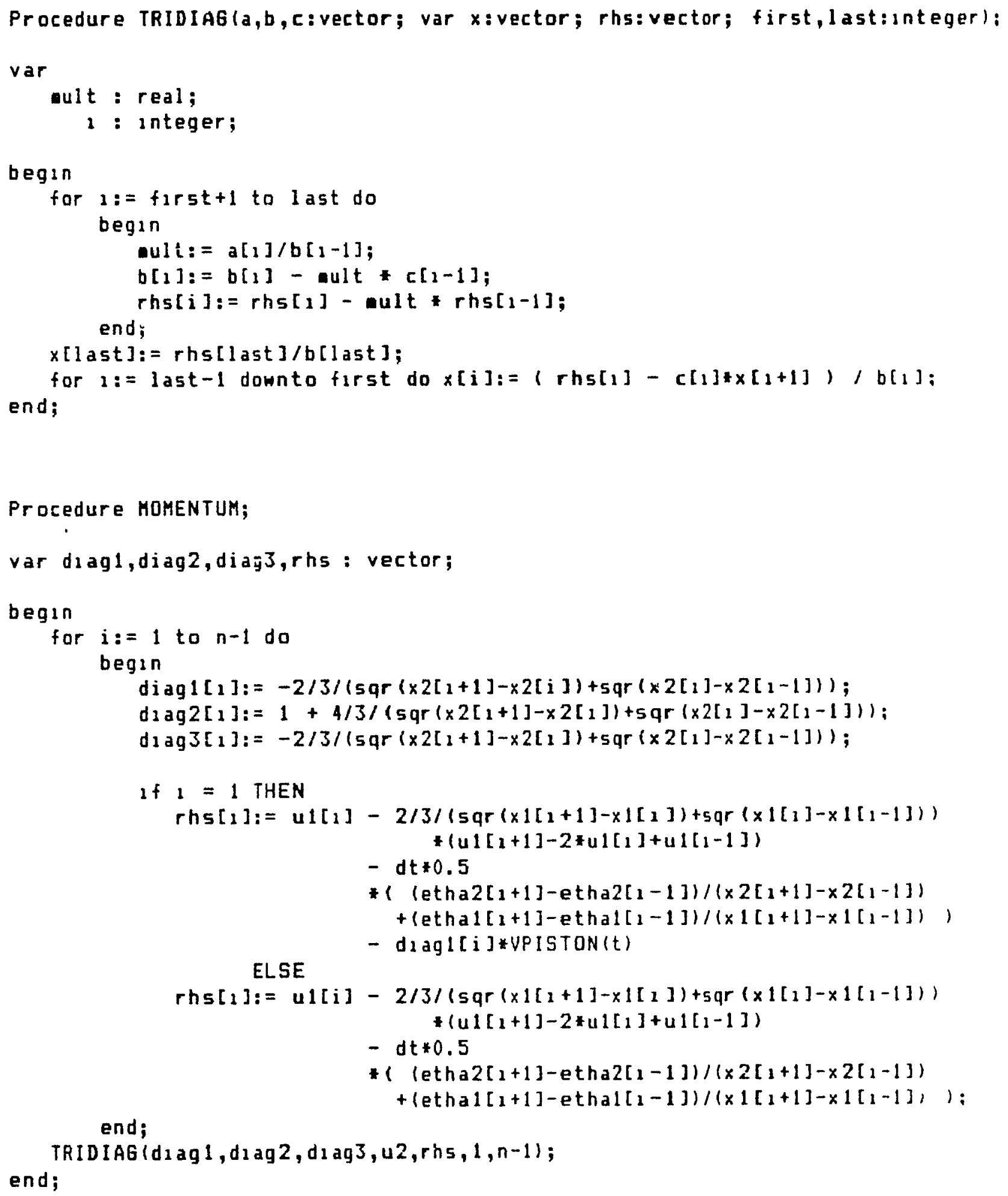

Procedure APPROX_INTERFACES;

begin

for $i:=0$ to $n$ do $\times 2[1]:=\times 1[1]+d t * u 1[1]$;

end; 
Procedure U_BC;

begin

$u 2[0]:=\operatorname{VPISTON}(t) ;$

$u 2[n]:=0.0$;

end;

Procedure INTERFACES;

begin

$\times 2[0]:=\times 1[0]+0.5 * d t *(u 1[0]+u 2[0]) ;$

for $1:=1$ to $n$ do

begin

$\left.\times 2[1]:=\times 1[1]+0.5 x d t \neq\left(u[]_{1}\right]+u 2[1]\right) ;$

if $(\times 2[1-1]<=g 1)$ and $(\times 2[1]>g 1)$

then if $(g 1-x 2[1-1])<=(\times 2[1]-g 1)$ then gaugel:= $1-1$

else gaugel: = 1;

If $(\times 2[1-1]<=g 2)$ and $(\times 2[1]>g 2)$

then if $(g 2-\times 2[1-1])<=(x 2[1]-g 2)$ then gauge $2:=1-1$

else gauge $2:=1$;

If $(\times 2[1-1](=g 3)$ and $(\times 2[1]>g 3)$

then $1 f(g 3-x 2[1-1])<=(x 2[i]-g 3)$ then gauge $3:=1-1$

else gauge $3:=1$;

if $(x 2[1-1]<=94)$ and $(\times 2[1]>94)$

then $1 f(94-x 2[1-1])<=(x 2[1]-94)$ then gauge $4:=1-1$

else gauge $4:=1$;

$1+(\times 2[1-1]<=95)$ and $(\times 2[1]>95)$

then if $(95-x 2[1-1])<=(x 2[1]-95)$ then gauges:= $1-1$

else gauges:= 1;

if $(\times 2[1-1]<=96)$ and $(\times 2[1]>96)$

then if $(96-x 2[1-1])<=(x 2[i]-96)$ then gaugeb: $=1-1$

else gaugeb: = 1;

If $(\times 2[i-1]\langle=g 7)$ and $(\times 2[1]>g 7)$

then if $(g 7-\times 2[1-1\})<=(\times 2[1]-97)$ then gauge $7:=1-1$

else gauge7: = 1;

If $(\times 2[1-1]\langle=g 8)$ and $(\times 2[1]>g 8)$

then if $(98-\times 2[1-1])\langle=(\times 2[1]-g 8)$ then gauged: $=1-1$

else gauger: = 1;

end;

end;

Procedure DRAWEED;

var $\times 1$ : real;

begin

HiRes:

Graphw1 ndow (50,40,589, 159);

GoToxy $(\theta, 23) ; m r$ teln ('TIME = , t:5:2,

piston VELOCITY $\left.={ }^{\prime}, \operatorname{VPISTON}(t): 5: 3,{ }^{\circ} \quad \operatorname{ACC}={ }^{\circ}, \operatorname{ACCPISTON}(t): 5: 3\right):$

$d r a w(r o u n d(x 2[0] /$ totald $\$ 540), 0$, round $(x 2[0] /$ totald $\$ 540), 100,1)$;

$x 1:=$ last $x /$ totald $\neq 540$;

draw(round $(x 1), 100,540,100,1)$;

end: 
for $1:=0$ to $n$ do ethal $[1]:=$ etha $2[1]$;

for $1:=0$ to $n$ do $u 1[i]:=u 2[1]$;

for $1:=0$ to $n$ do $\times 1[1]:=\times 2[i]$;

end;

close $(d 15 k)$;

END. 


\title{
Program SUBWED
}

\section{Purpose of the program}

Modelling of nonlinear, dispersive, shallow-water waves qenerated ind channel by the notion of a submerged wedge.

\section{Definition of the constants}

\author{
$n$ : Total number of spatial grid points $(1=0,1 \ldots \ldots)$. \\ $n 1: n-1$. \\ $d x$ : Spatial mesh size (constant). \\ dt : Temporal mesh $51 z e$. \\ taax : Total duration of the simulation. \\ endaotion: Total duration of the bed motion. \\ vstar : Amplitude of the horizontal, sinusoidal velocity of the \\ wave generating device. \\ slope : Constant slope of the submerged wedge. \\ xsin : Parameter detining the sinusoidal velocity of the \\ bed, $x \sin =\tau_{2}-\tau_{1}$. \\ eu : Implacitness parameter for the depth-averaged velocity. \\ en : Implicitness parameter for the wave amplitude. \\ eh : Implicitness parameter for the bed position.
}

\section{Definition of the global varlables}

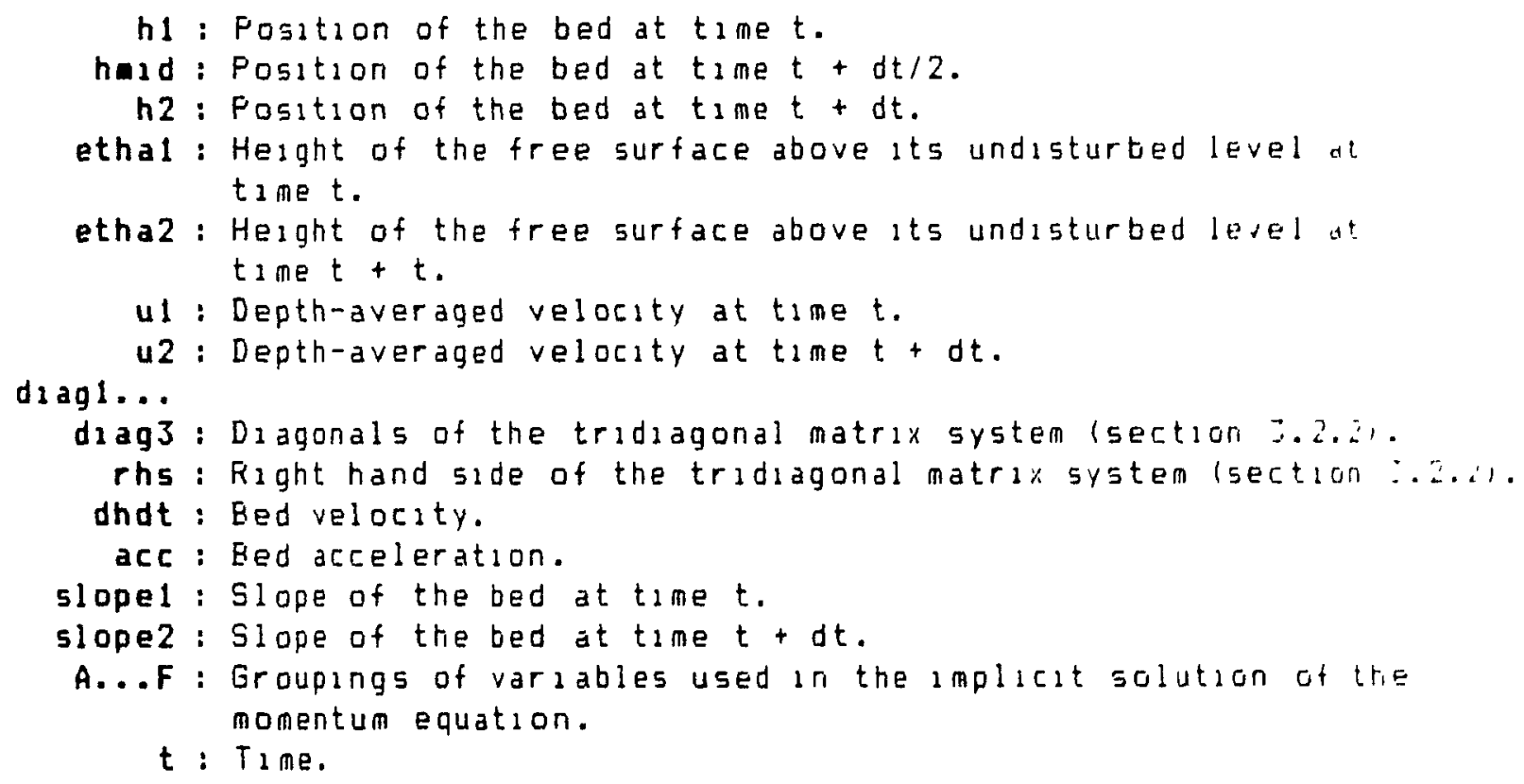




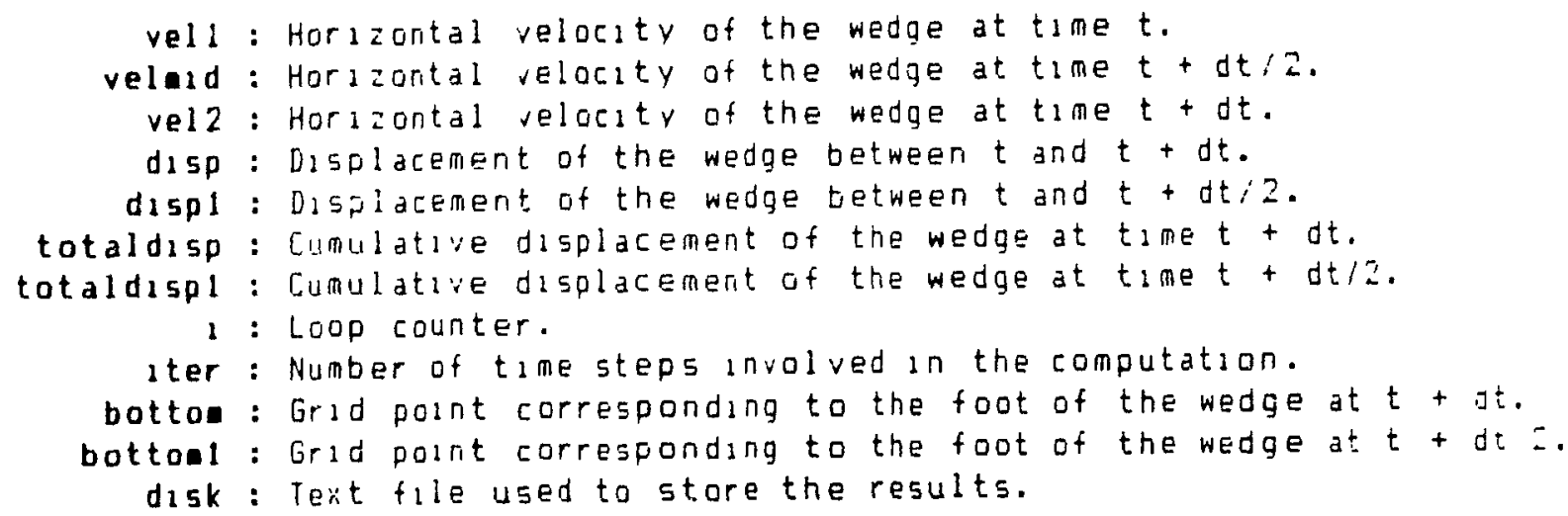

\section{Program IIsting}

Progran SUBWED (input,output);

uses crt,graph3;

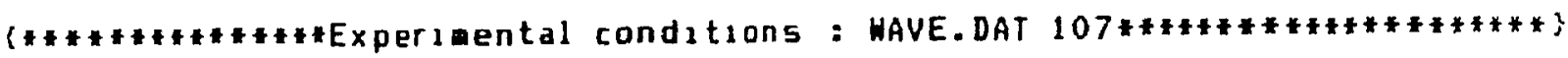

CONST

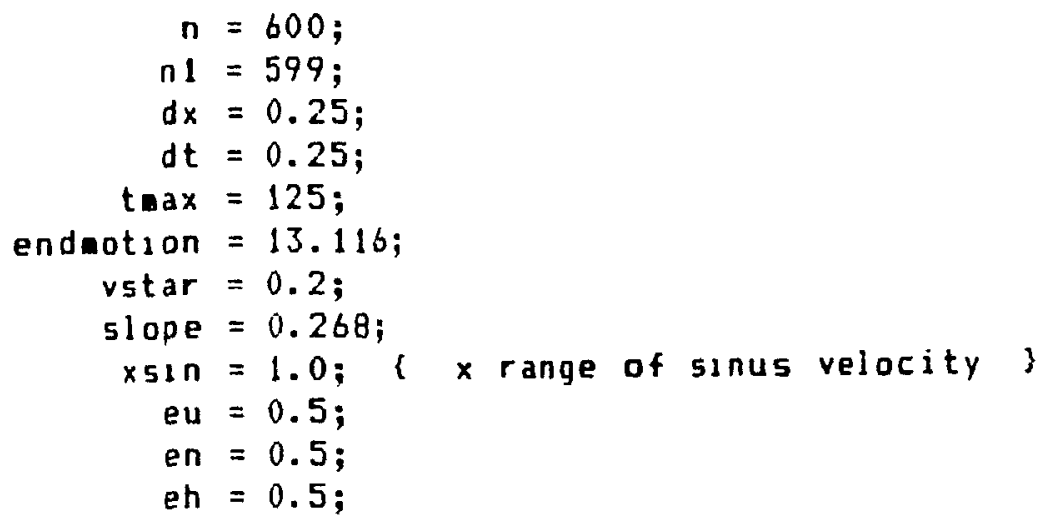

TYPE

vector = array $[0 . . n]$ of rea];

VAR

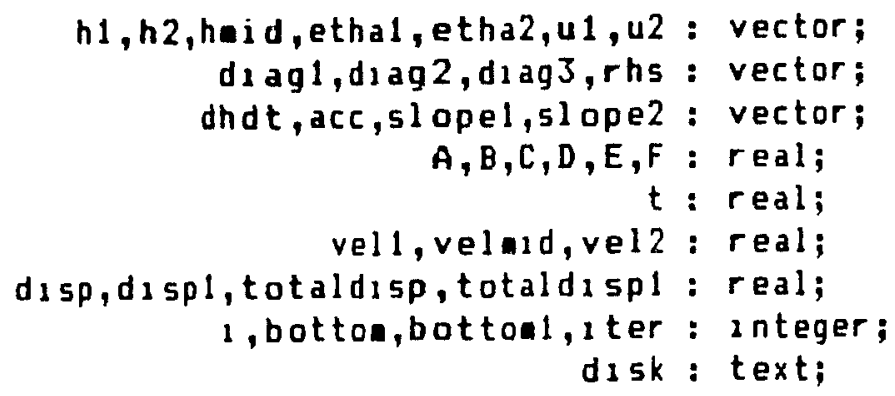




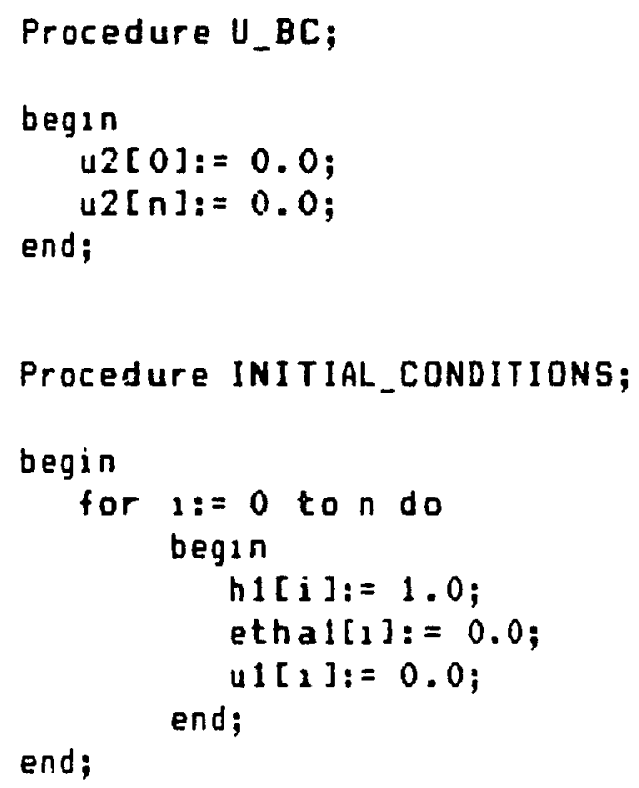




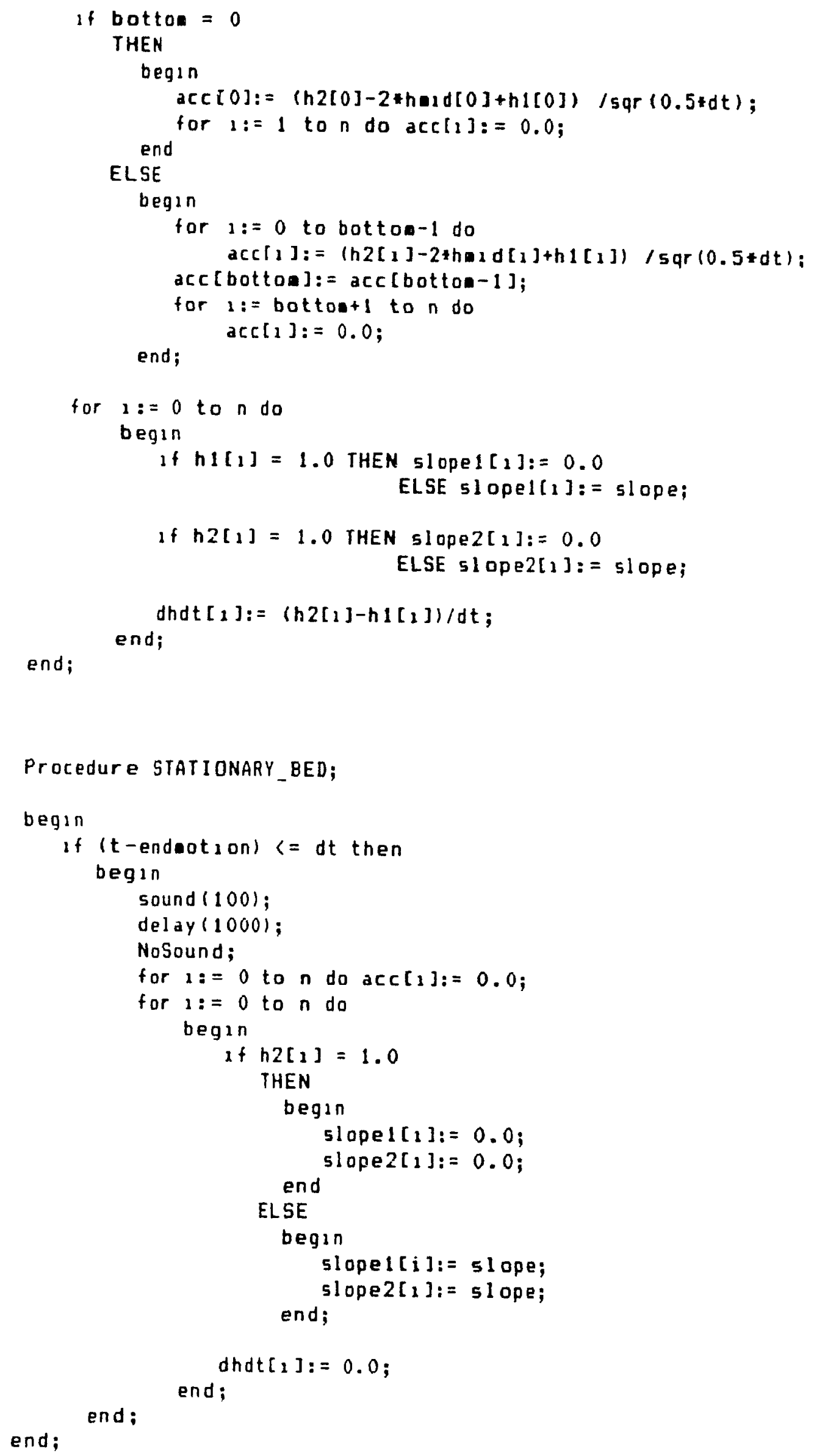




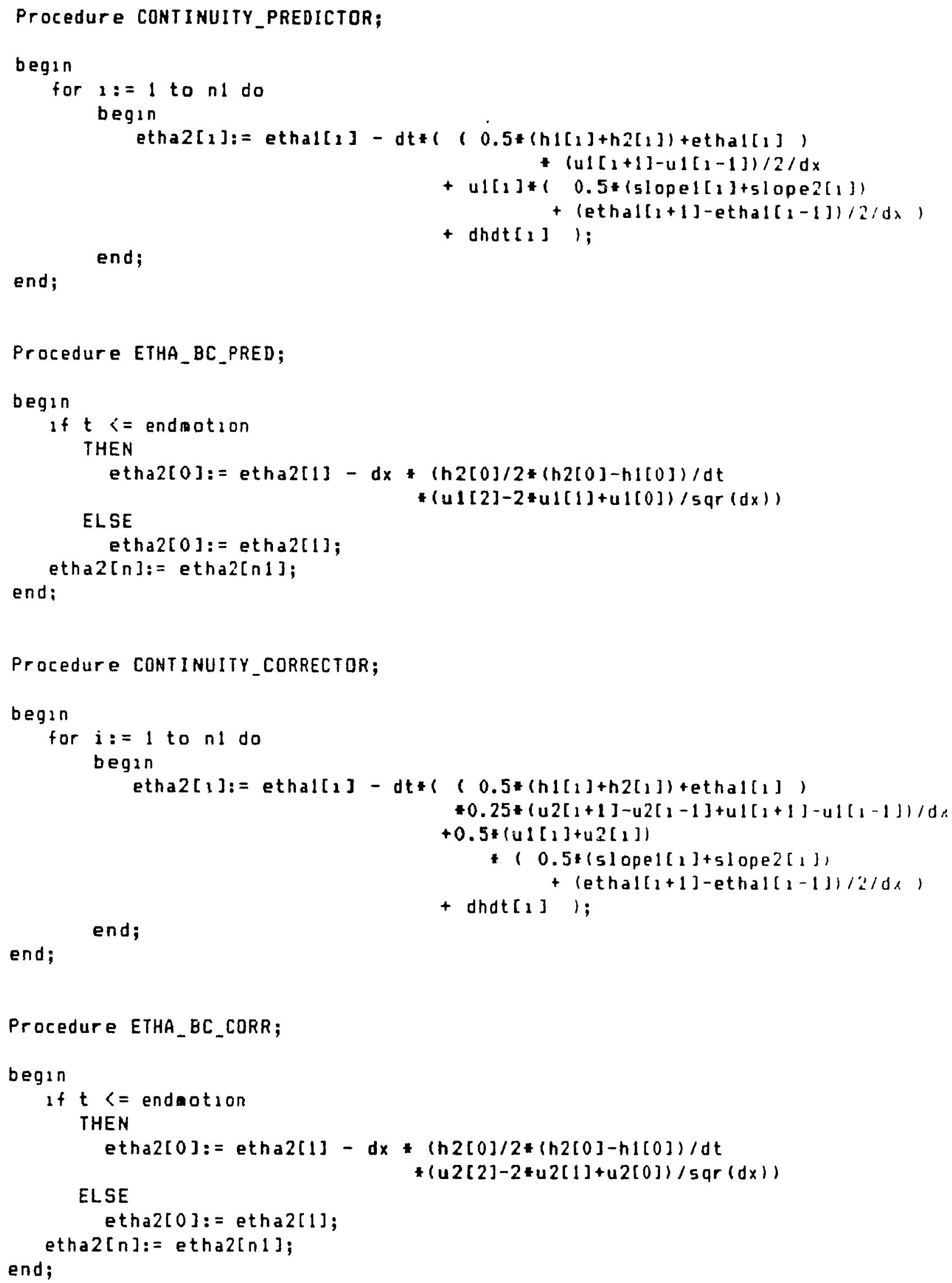




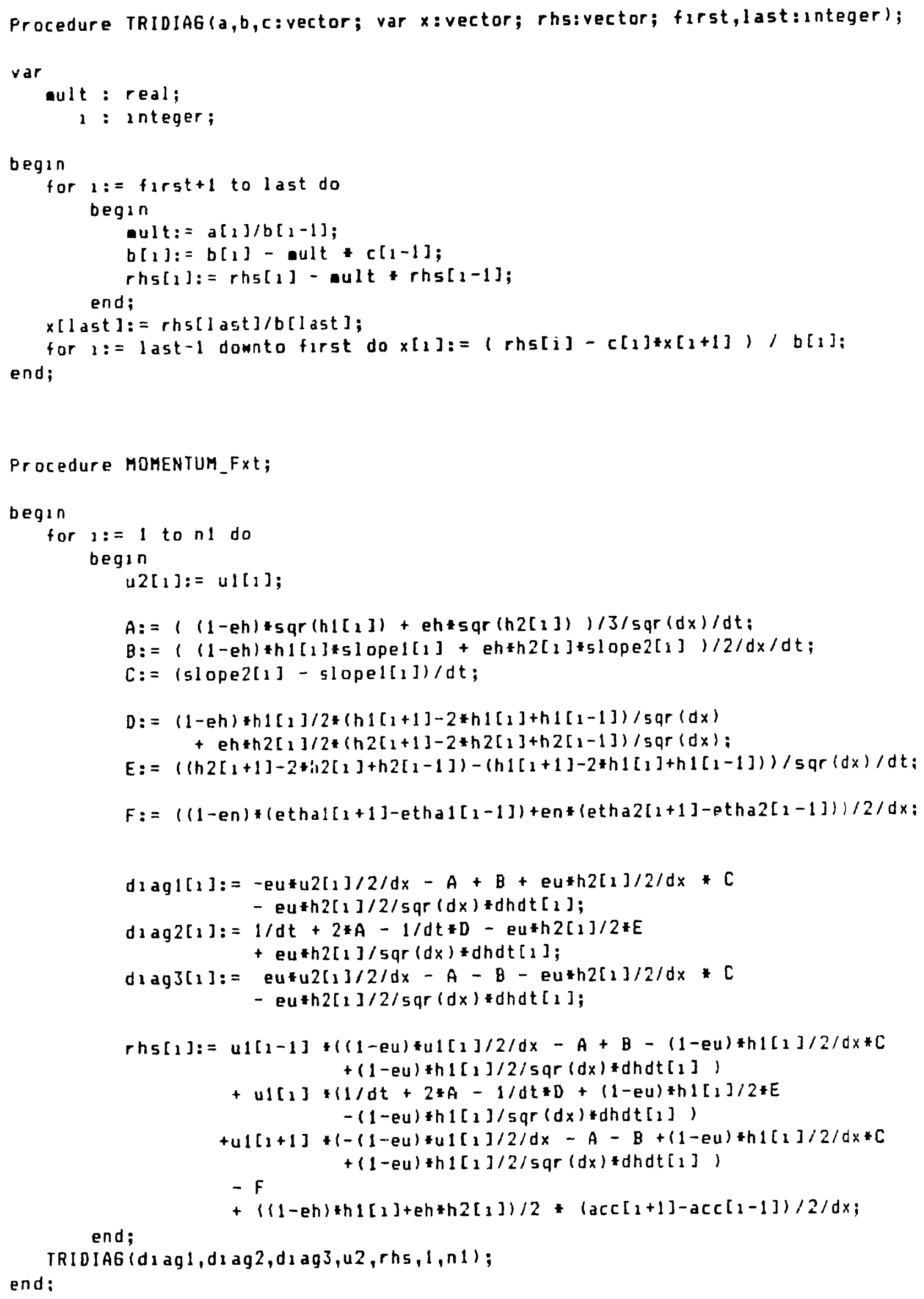


Procedure MOHENTUH_FX;

begin

for $1:=1$ to $n 1$ do

begin

$u 2[1]:=u 1[1]$;

$A:=\operatorname{sqr}(\ln 2[1]) / 3 / 5 q r(d x) / d t ;$

$B:=h 2[$ i $] \neq 5 l$ upe $2[\mathrm{l}] / 2 / d x / d t ;$

$D:=h 2[1] / 2+(h 2[1+1]-2 * h 2[1]+h 2[1-1]) / \operatorname{sgr}(d x) ;$

$F:=((1-e n) *(e \operatorname{tha}[\{1+1]-\operatorname{etha}][1-1])+e n *(\operatorname{etha} 2[1+1]-\operatorname{etha} 2[1-1])) / 2$, d: :

daggl[1]: = -eu*u2[1]/2/dx-A+Bi

diag2[1]: $=1 / d t+2 * A-1 / d t * D$;

diagJ[1]: = eutu2[1]/2/dx-A-B;

$\operatorname{rhs}[1]:=\quad u 1[1-1]+((1-e u) * u 1[1] / 2 / d x-A+B)$

$+u 1[1] *(1 / d t+2 * A-1 / d t+D)$

$+u 1[1+1]+(-(1-e u) * u 1[1] / 2 / d x-A-B)$

$-\mathrm{F} ;$

end;

TRIDIAG (diagl, diag2,dzag $3, u 2$, rhs $, 1, n 1)$;

end;

Procedure MOMENTUM:

begin

if $t<=$ endmotion THEN MOHENTUM_f $x t$

ELSE MOMENTUM_FX;

end;

Procedure DRAWBED(vivector; no_points:integer);

var $x 1, x_{-, y 1, y 2}:$ real;

color : Integer;

begin

$H_{1} R_{t}=$,

GraphHindow $(50,40,589,159)$;

6oToXY $(35,20) ; m r i t e l n(\cdot t=\cdot, t: 6: 3)$;

( for 1:= 0 to no points do draw(round(i/no_points $\$ 5401,90$,

for $1:=0$ to no_points-1 do round ( $1 /$ no_point5\$540),20,1);

begin

$\times 1:=1 /$ no_points $\$ 540$;

$y 1:=v[1] * 30$;

$\times 2:=(1+1) /$ no_points $* 540$;

y $2:=v[1+1] * 30$;

draw(round (1/no_points $\$ 540), 60$, round (1/no_points\$540),0,1);

draw (round $(x 1), 60+r$ ound $(y 1)$, round $(x 2), 60+r$ ound $(y 2) .1)$; end;

end; 
Procedure DRAWSURFACE (v:vector; no_points: integer);

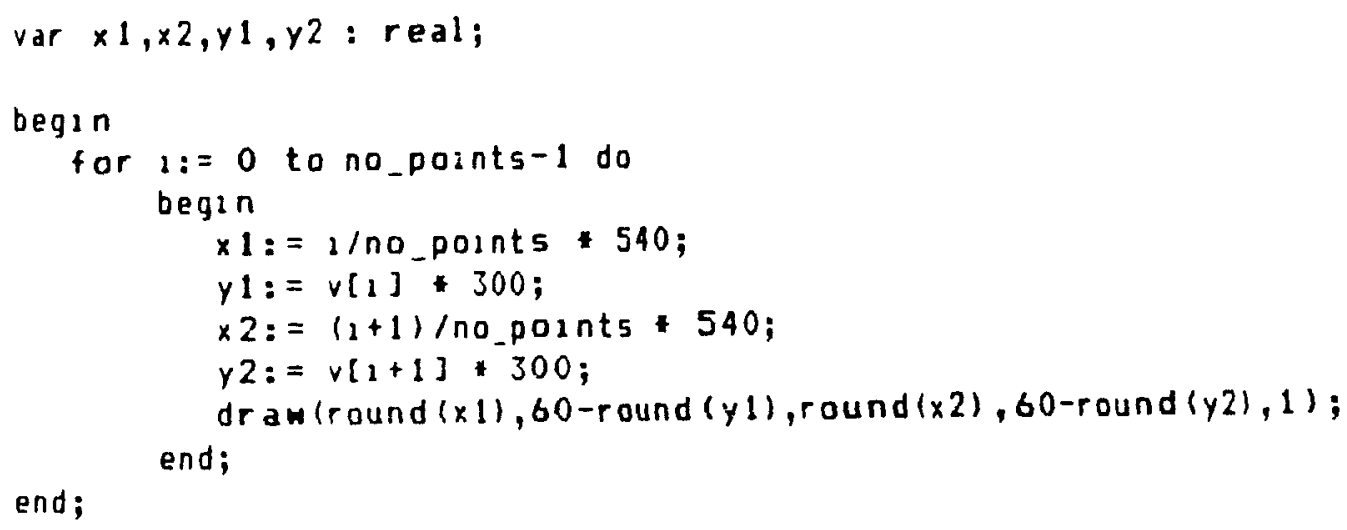




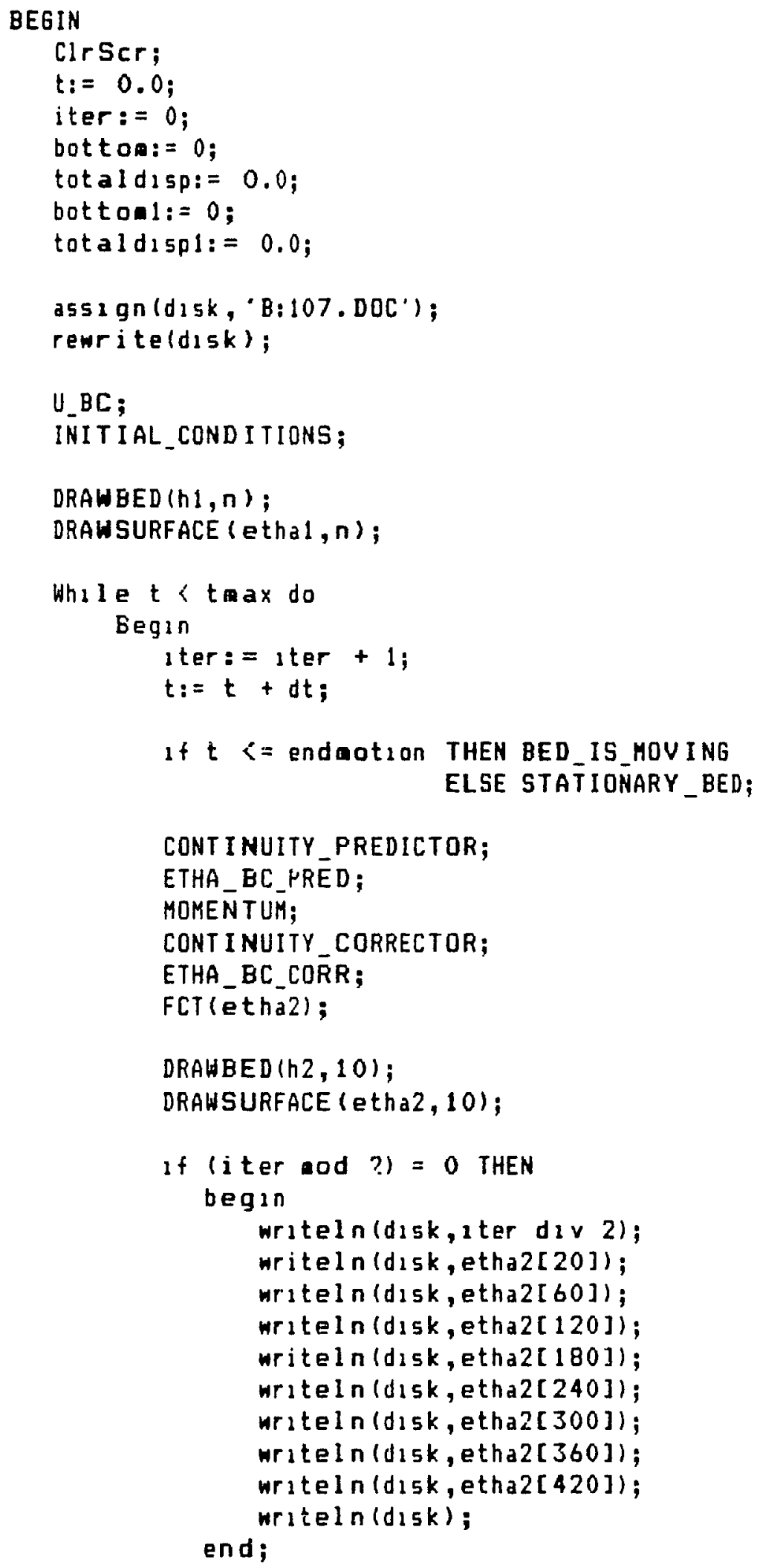




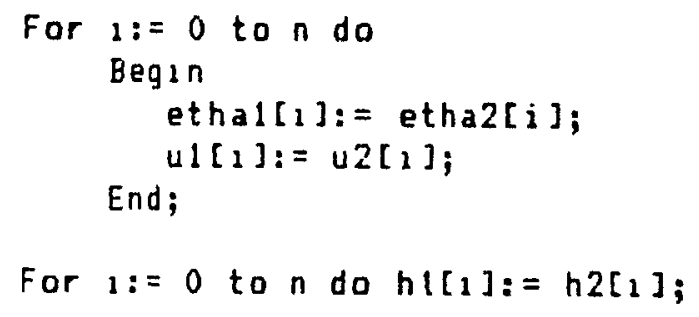




\section{Program SHELF}

\section{Purpose of the program}

Modelling of nonlinear, dispersive, shallow-water waves generated in a channel by the motion of a shelf.

\section{Definition of the constants}

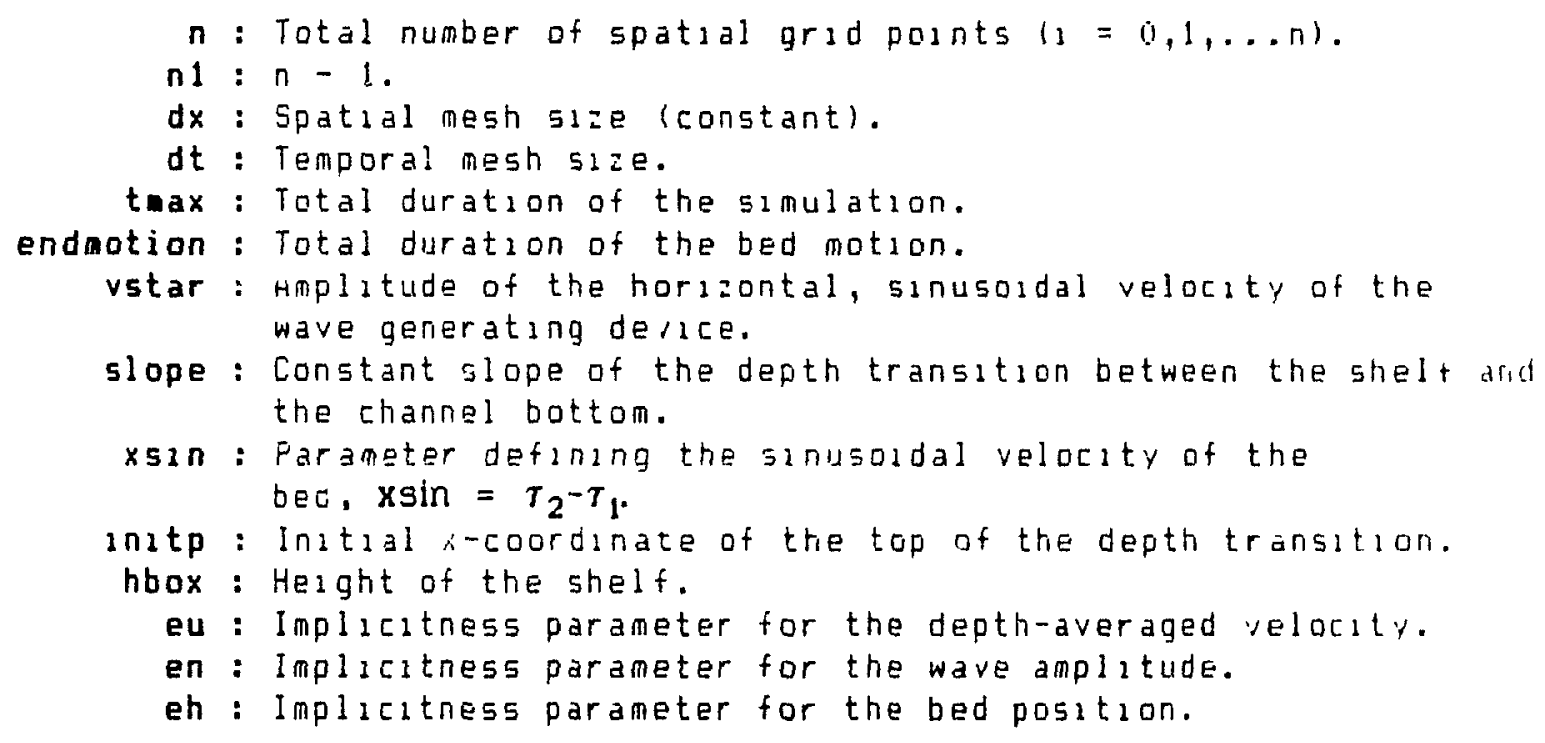

\section{Definition of the global variables}

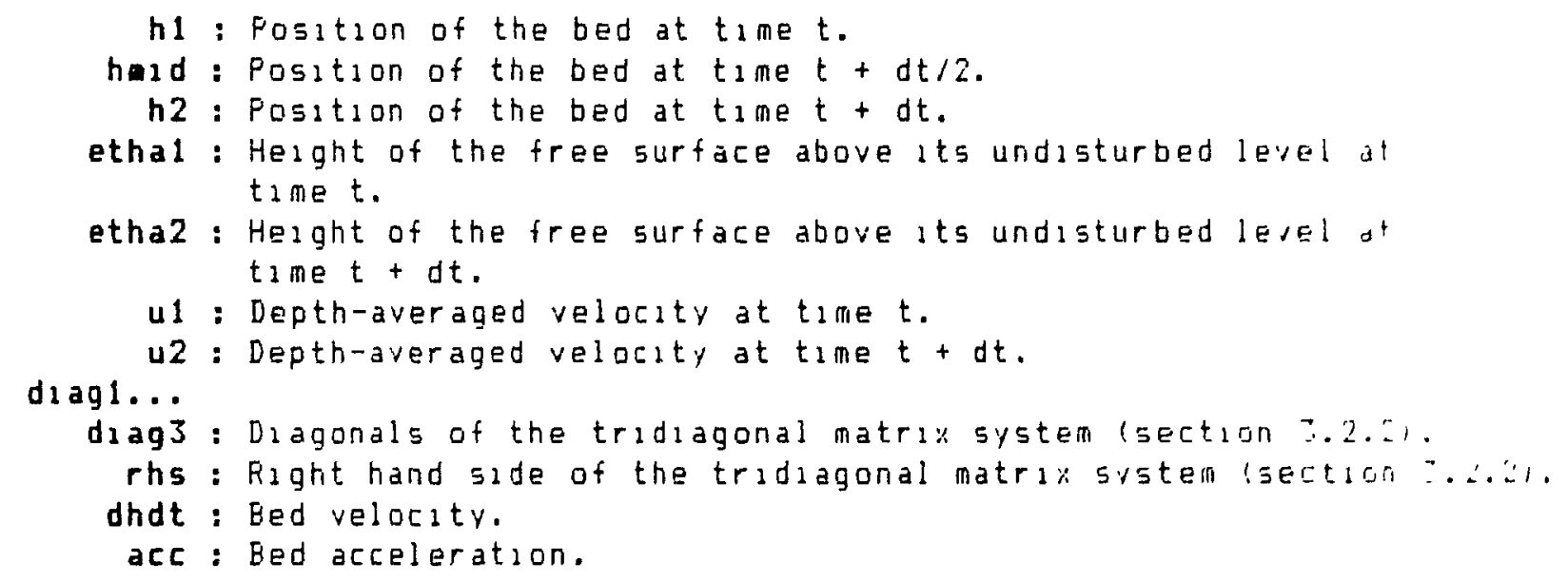




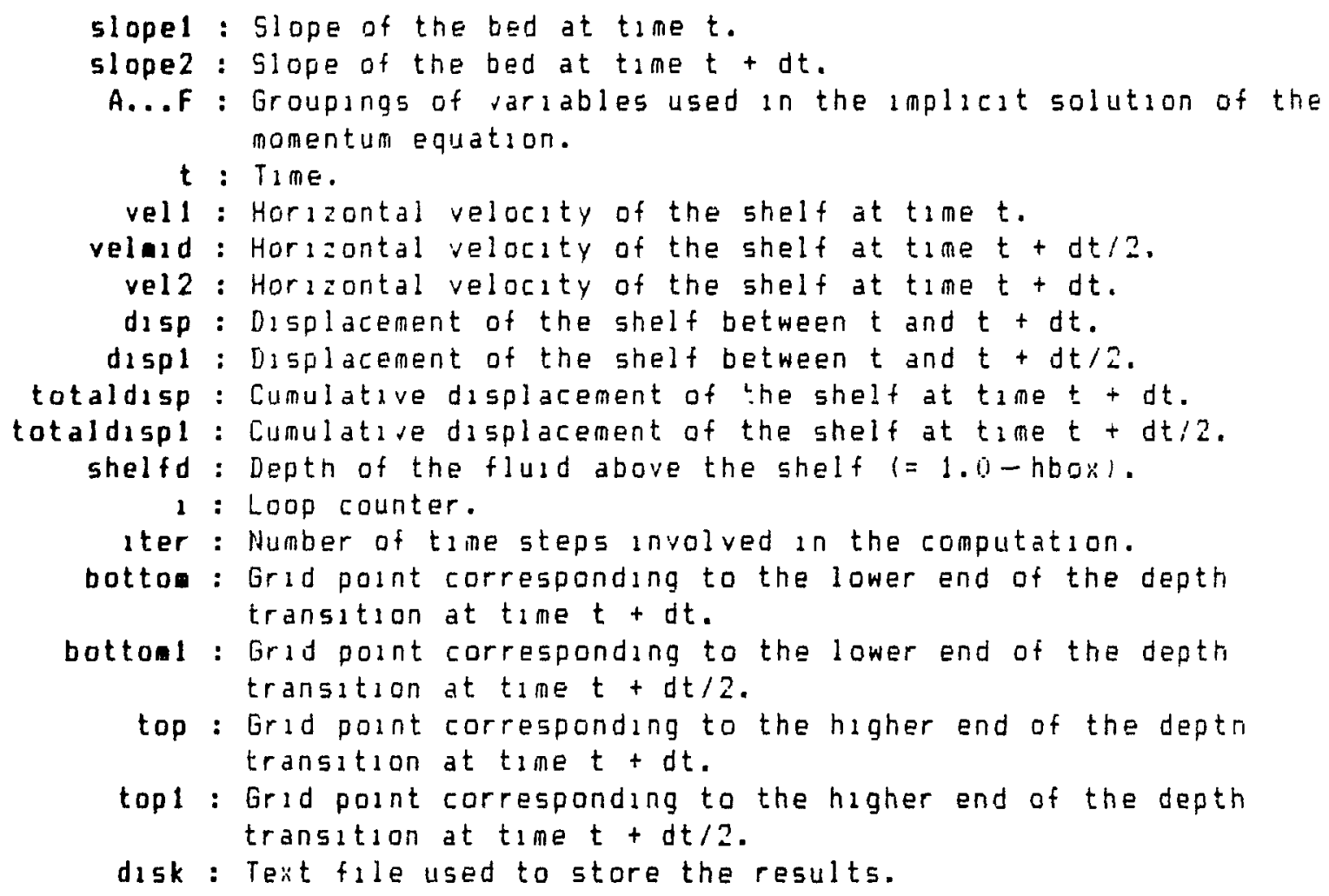

\section{Program 11sting}

Prograa SHELF (1nput, output);

uses crt,graph3;

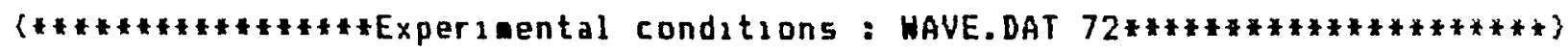

CONST

$$
\begin{aligned}
n & =500 ; \\
n 1 & =499 ; \\
d x & =0.25 ; \\
d t & =0.25 ; \\
\operatorname{tadx} & =120 ; \\
\text { endation } & =13.0 ; \\
v 5 t a r & =0.2 ; \\
\text { slope } & =0.577 ; \\
\text { xs } & =1.0 ; \\
1 n 1 t p & =5.0 ; \\
\text { hbox } & =0.33333 ; \\
\text { eu } & =0.5 ; \\
\text { en } & =0.5 ; \\
\text { eh } & =0.5 ;
\end{aligned}
$$

TYPE

$$
\text { vector = array }[0 . . n] \text { of real; }
$$


VAR

$$
\begin{aligned}
& \text { h1,h2,høid, etha1, etha2,u1,u2 : vector; } \\
& \text { dlag1,dlag2,diag3, rhs : vector; } \\
& \text { ohdt,acc,slopel,slope2 : vector; } \\
& A, B, C, D, E, F \text { : real; } \\
& t \text { : real; } \\
& \text { vell, velud,vel 2 : real; } \\
& \text { disp,displ, totaldisp,totaldispl i real; } \\
& \text { shelfd : real; } \\
& \text { 1, bottoa,bottoal,top,topl,1ter: integer; } \\
& \text { disk : text; }
\end{aligned}
$$

Procedure U_BC:

begin

$\mathrm{u} 2[0]:=0.0$

$u 2[n]:=0.0$

end:

Procedure INITIAL_CONDITIONS;

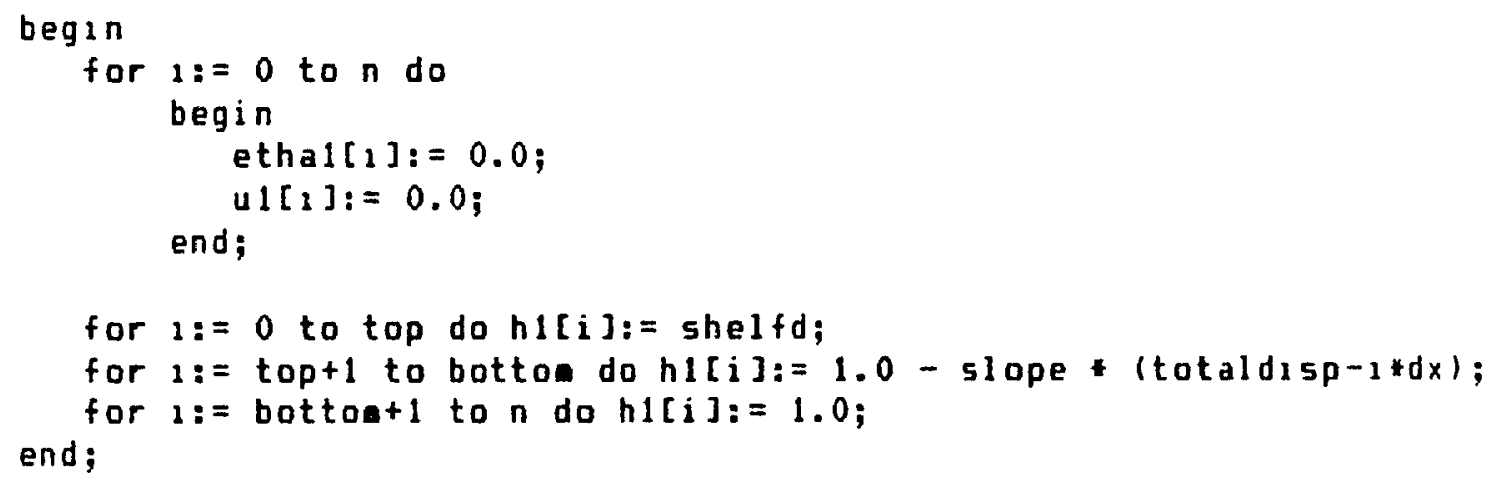

Procedure MOVE_BED;

began

for $1:=0$ to top do $h 2[i]:=$ shelfd;

for $1:=$ top+l to botton do $h 2[i]:=1.0-$ slope ( $(\operatorname{totald} 15 p-2 * d x)$;

for $1:=$ bottoes to $n$ do $h 2[1]:=1.0$;

for $1:=0$ to top 1 do heid[1]:= shelfd;

for $1:=\operatorname{top} 1+1$ to bottonl do hald[1]:=1.0 - slope * (totaldasp1-1*d 1$)$;

for $1:=$ bottonlt to $n$ do hasd $[1]:=1.0$; end;

Procedure BED_IS_MOVING;

begin

vel1:= vstar $\$ \sin (0.5 * p 1-(0.5 *$ endnotion-(t-dt)) *xsin/endaotiontpl 1 ;

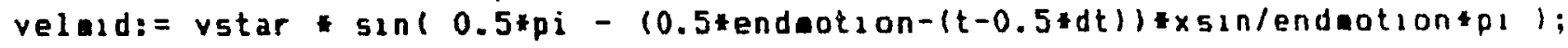
vel 2: = vstar $* \sin (0.5 * p i-(0.5 *$ endeotion-t)*xin/endaot 2 on*pl); 


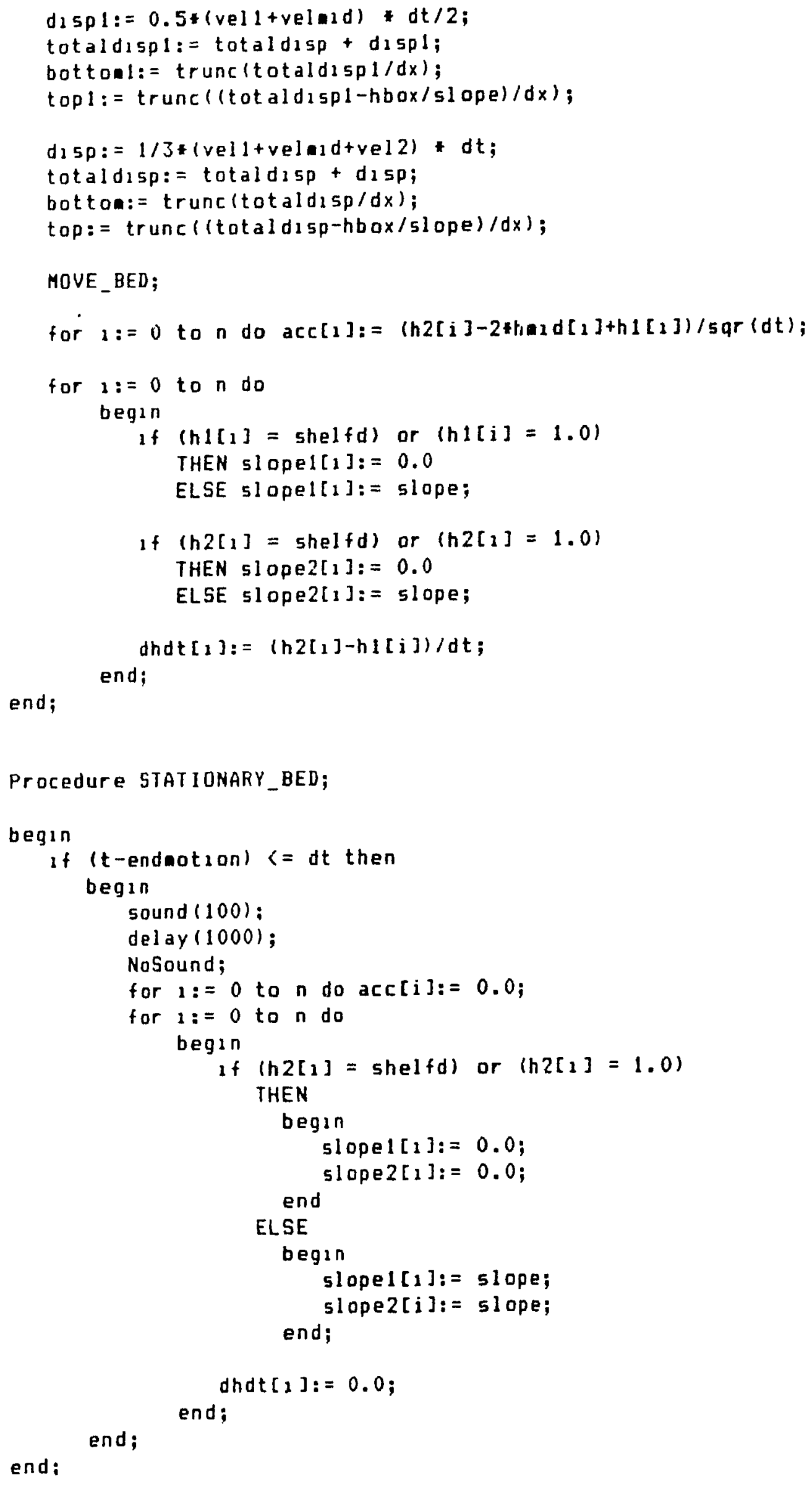




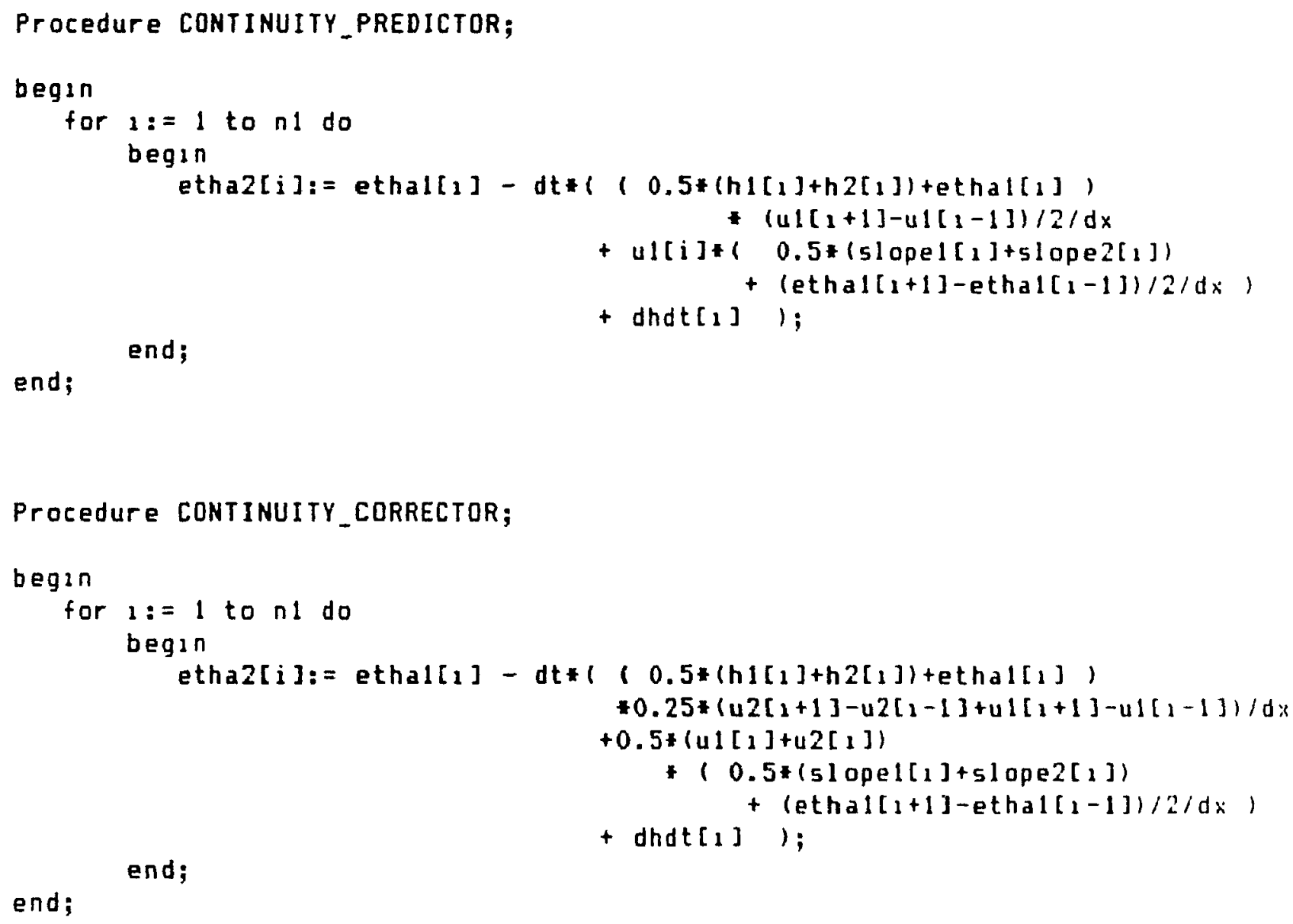




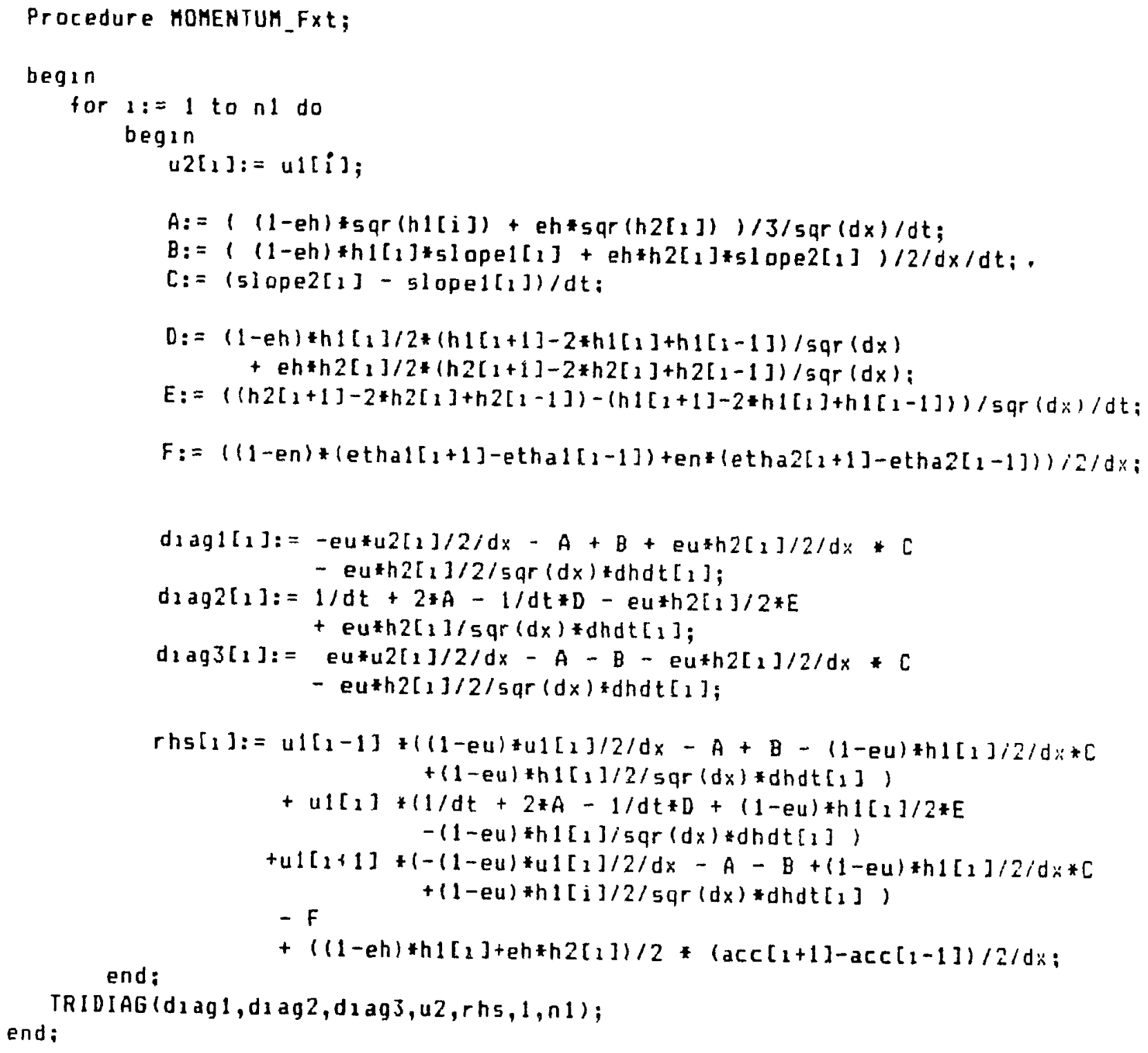

TRIDIAG (d) agl, diag $2, d 1$ ag $3, u 2, r h s, 1, n 1)$;

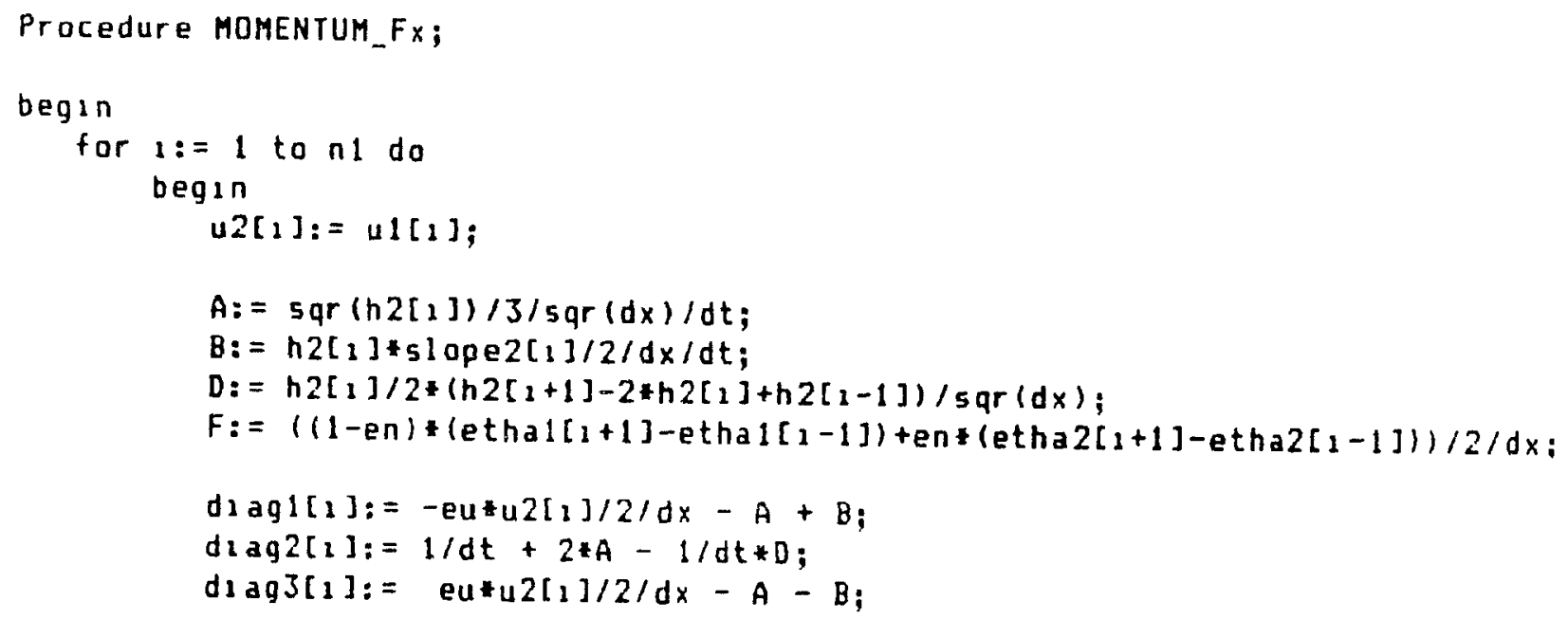




$$
\begin{aligned}
\operatorname{rhs}[1]:= & u t[1-1]+((1-e u)+u 1[1] / 2 / d x-A+B) \\
& +u 1[i]+(1 / d t+2+A-1 / d t+D) \\
& +u 1[1+1]+(-(1-e u)+u 1[1] / 2 / d x-A-B) \\
& -F
\end{aligned}
$$

end; end;

TRIDIAG (d)agl, d1 ag2, di ag $3, u 2$, rhs $, 1, n 1)$;

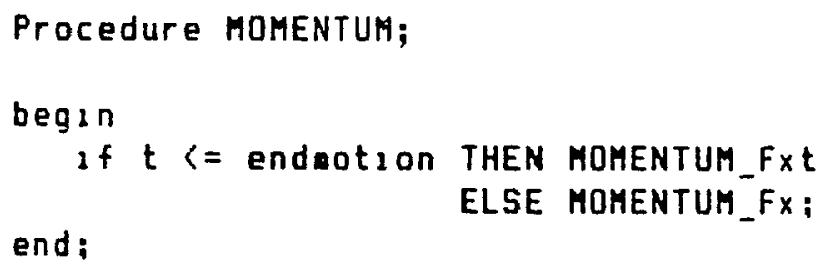

end:

Procedure DRAHBED(v:vector; no_points: integer);

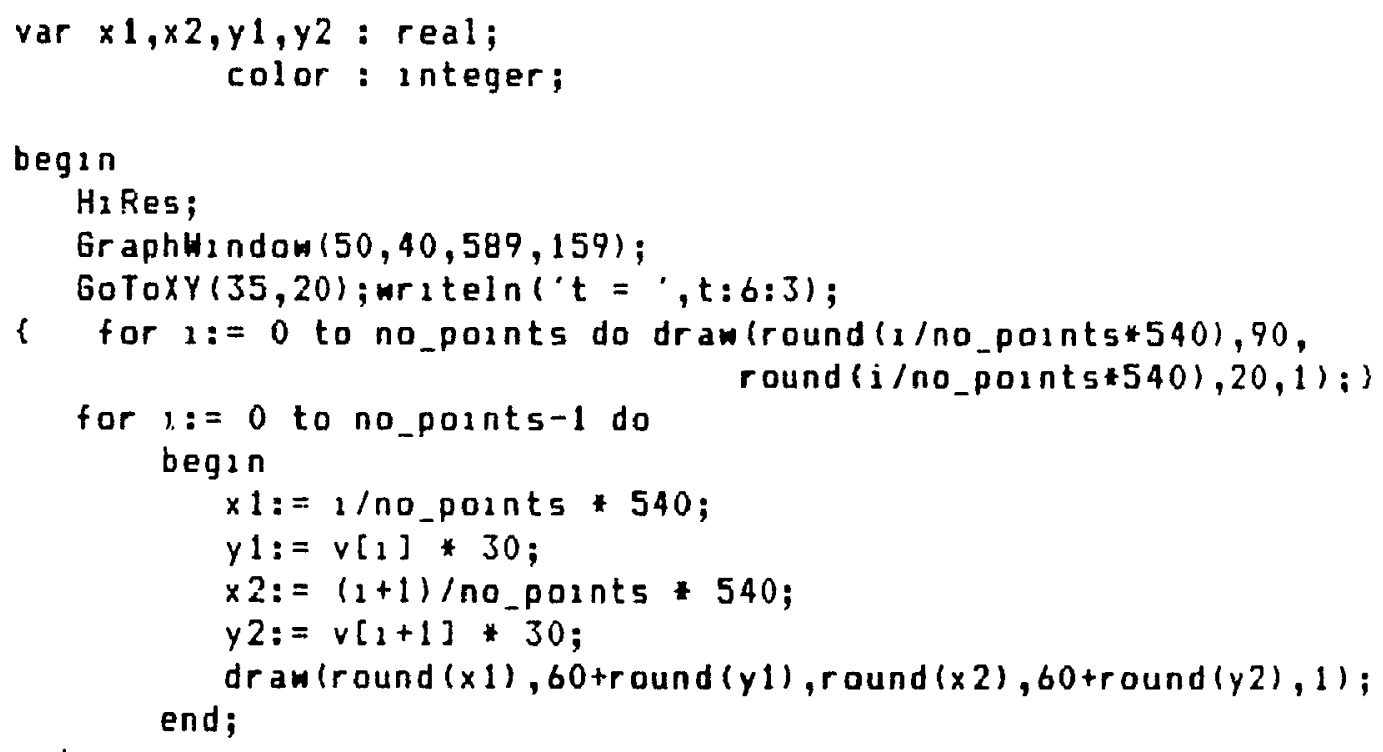

Procedure DRAHSURFACE(v: vector; no_points:1nteger);

$\operatorname{var} \times 1, \times 2, y 1, y 2$ : real;

began

for $1:=0$ to no_points-1 do begin 


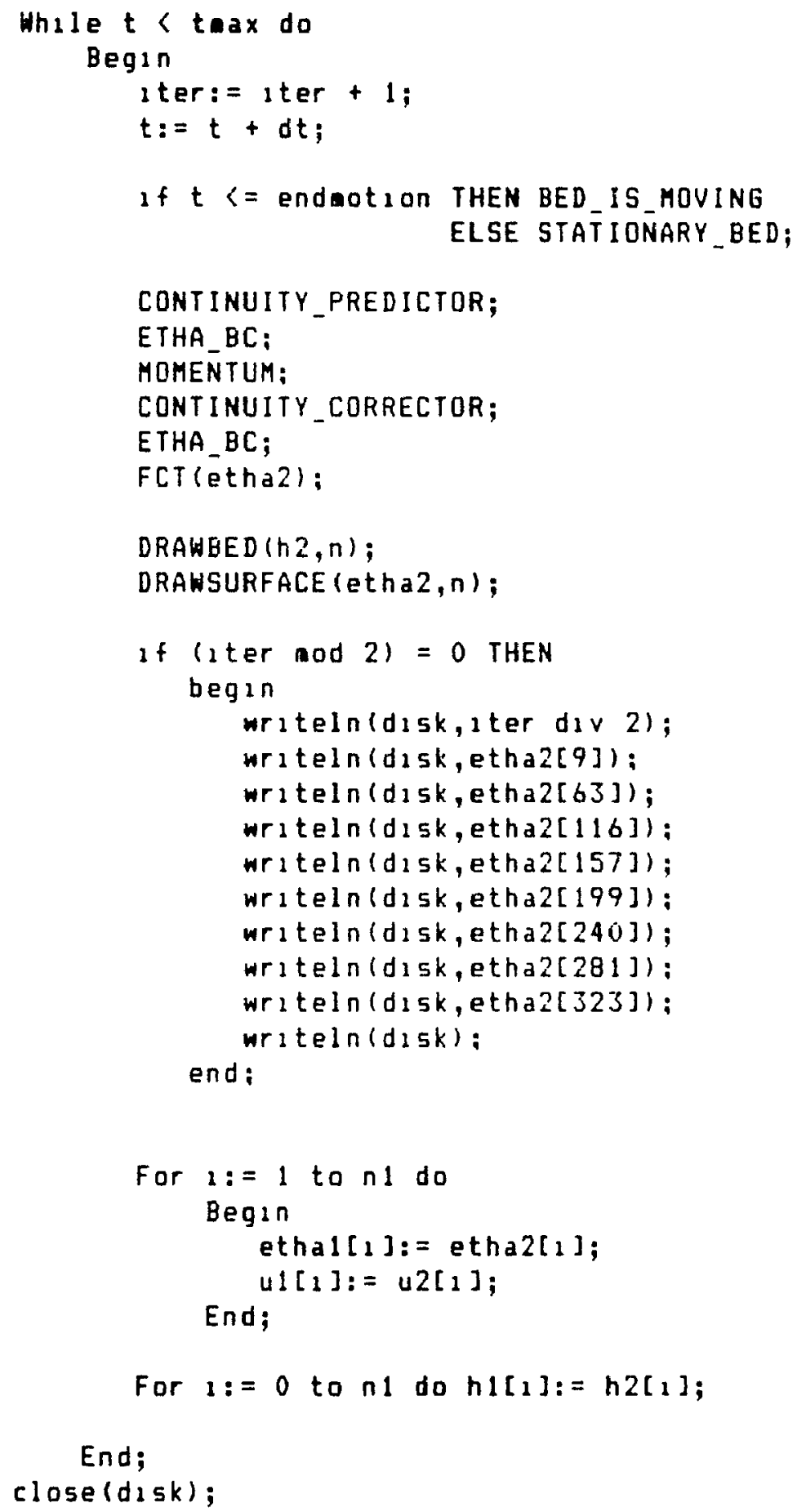




\section{Prog ram ROPLATE}

\section{Purpose of the program}

Modelisng of nonlinear, dispersive, shallow-water waves generated in a channel by the motion of a rotating plate.

\section{Definition of the constants}

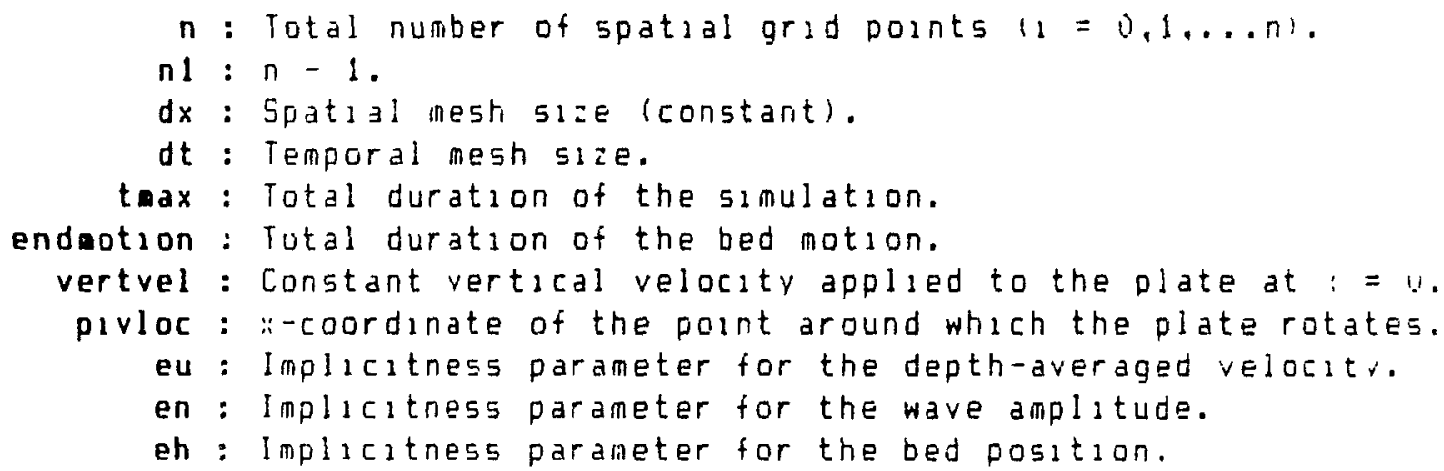

\section{Definition of the global variables}

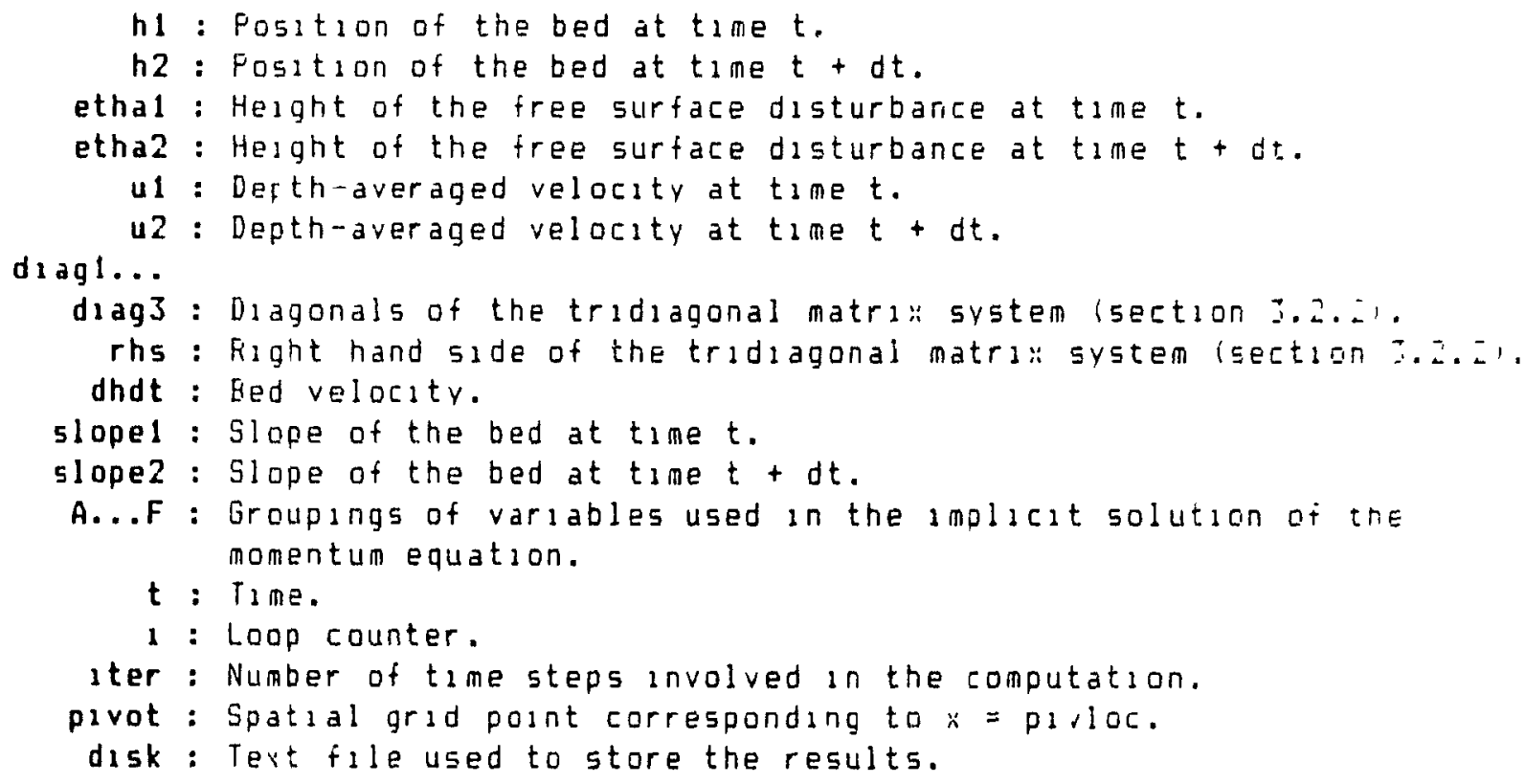




\section{Program listing}

Progran ROTATING_PLATE (1nput, output);

uses crt,graph3;

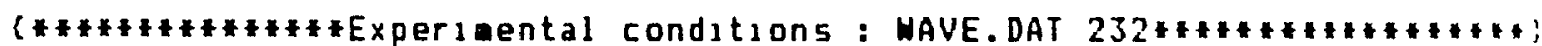

CONST

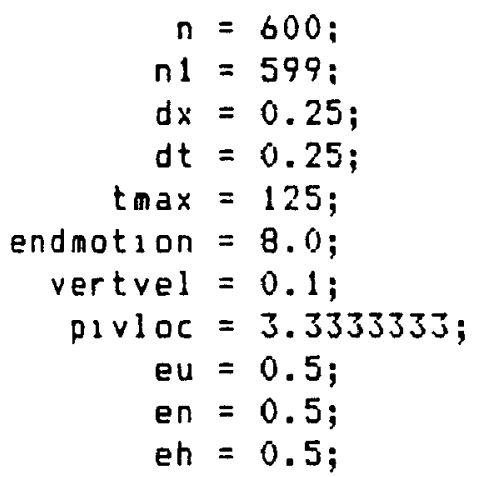

TYPE

vector = array $[0 \ldots n]$ of real;

VAR

$$
\begin{aligned}
& \text { h1,h2, ethal, etha2,ul,u2 : vector; } \\
& \text { 5lope1,5lope2, dhdt : vector: } \\
& \text { daagl,daag2,daag3,rhs : vector: } \\
& t \text { : real: } \\
& A, B, C, D, E, F \text { : real; } \\
& \text { 1, ater,pivot : integer; } \\
& \text { disk : text; }
\end{aligned}
$$

Procedure U_BC;

begin

$u 2[0]:=0.0$

$u 2[n]:=0.0 ;$

end;

Procedure INITIAL_CONDITIONS;

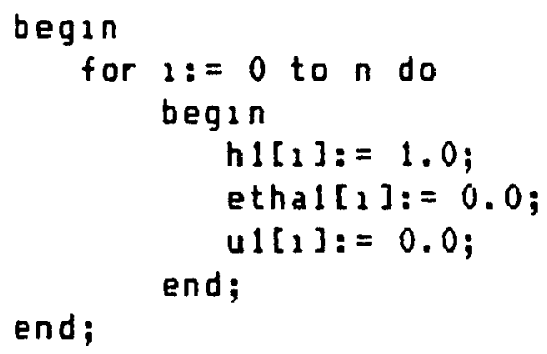




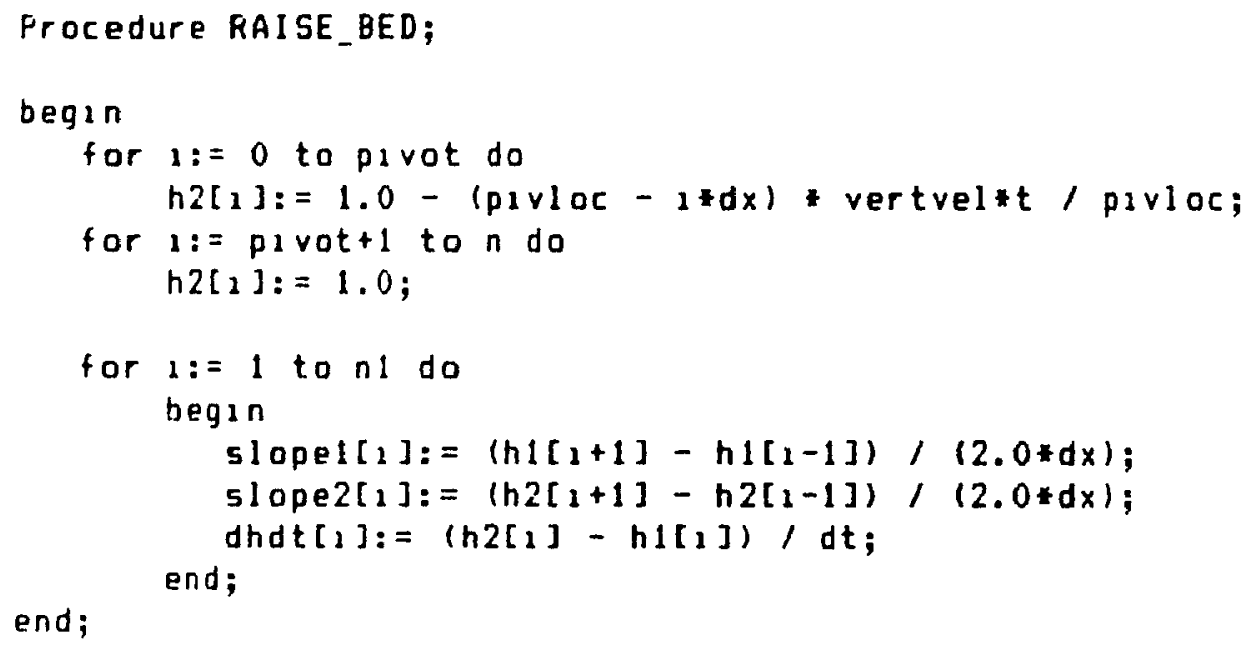

end; 


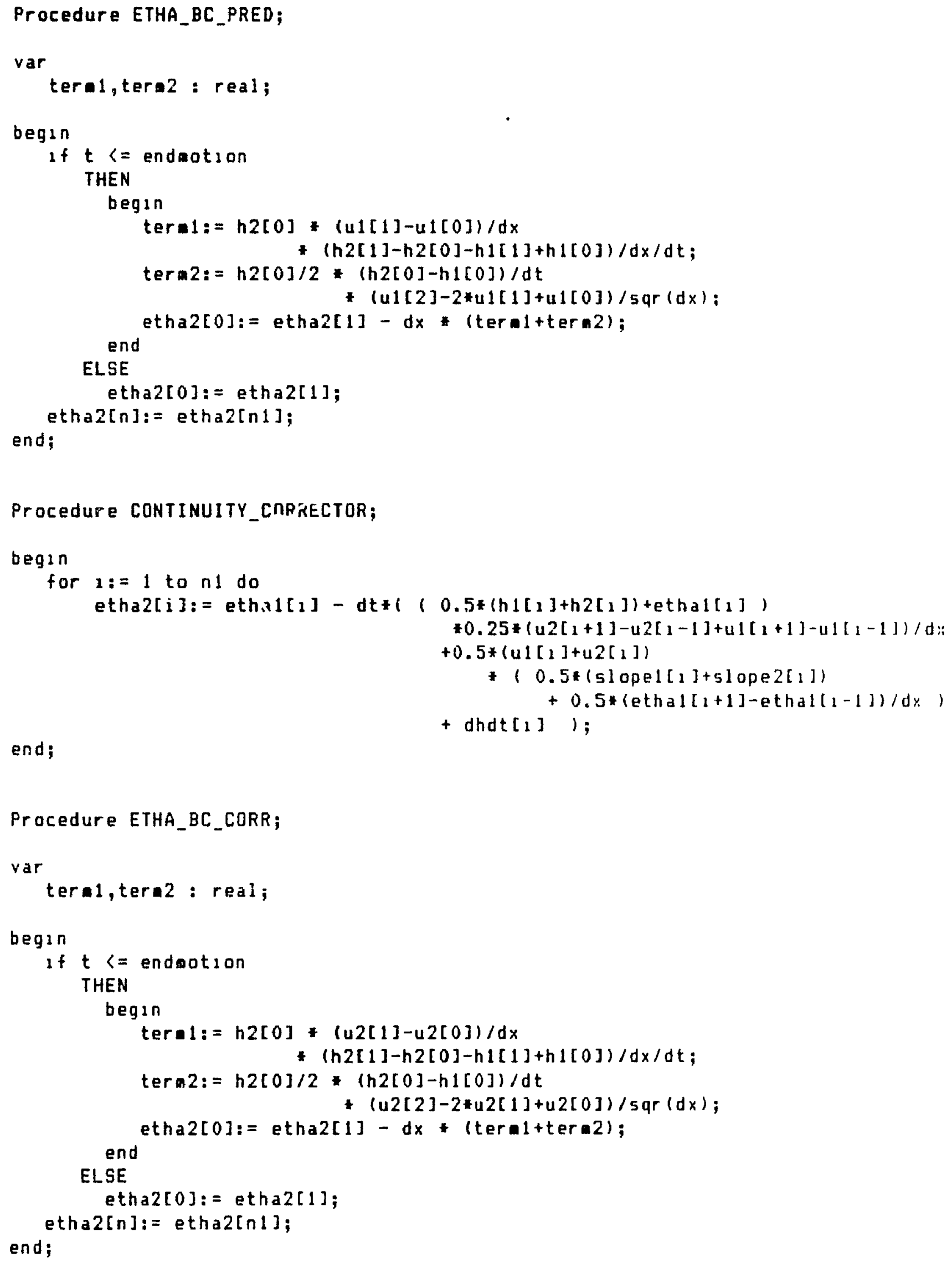




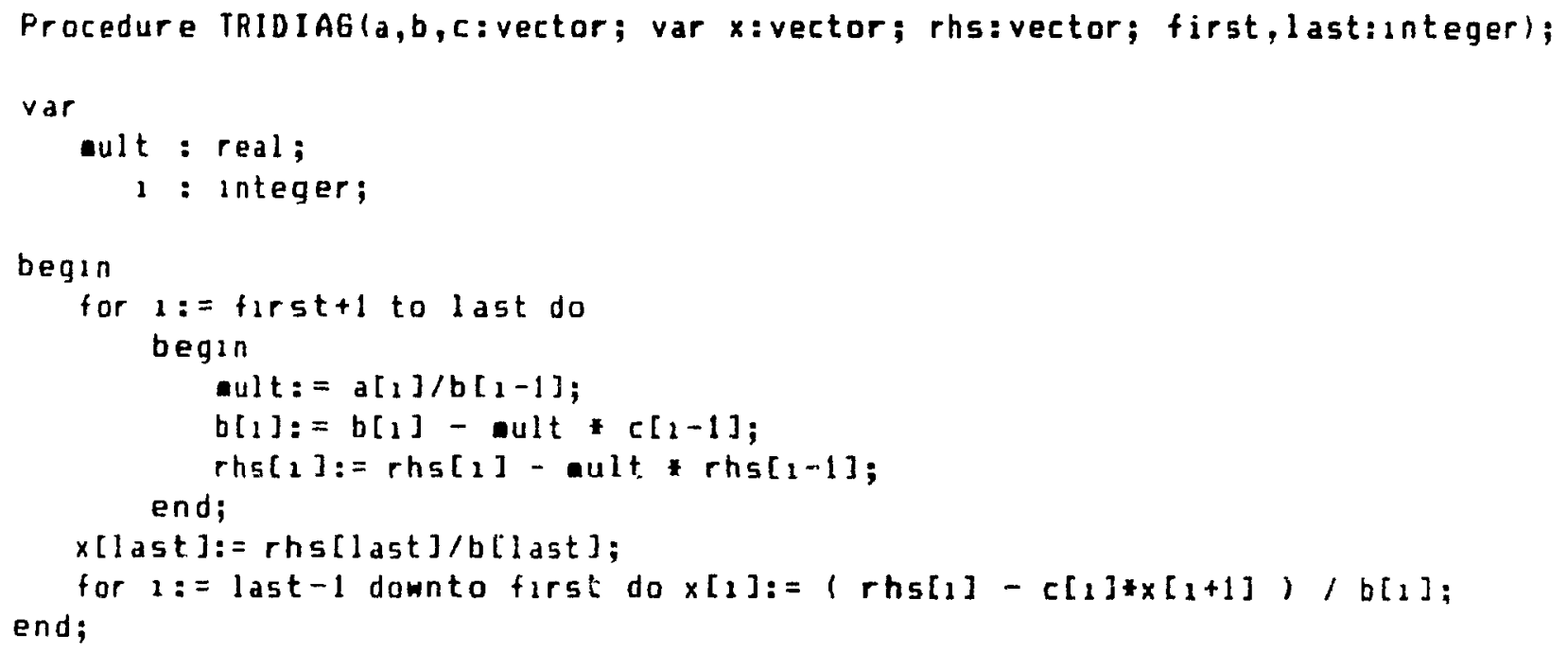


Pracedure MOMENTUH_Fx;

begin

for $1:=1$ to $n 1$ do

begin

$u 2[1]:=u 1[1] ;$

$A:=\operatorname{sqr}(h 2[1]) /(3 * 5 q r(d x) * d t) ;$

$B:=5$ lope $2[1] * h 2[i] /(4 * d x+d t)$;

$D:=h 2[1] / 2 *(h 2[1+1]-2 * h 2[1]+h 2[1-1]) / \operatorname{sqr}(d x)$;

$F:=((1-e n) *(e t h a 1[i+1]-e t h a 1[1-1])+e n *(e t h a 2[1+1]-e t h a 2[1-1])) / 2$, dx:

diagl[1]:= -eu*u2[1]/2/dx-A+B;

$d \operatorname{ag} 2[1]:=1 / d t+2 * A-1 / d t * D$;

dzag $[i]:=$ eu*u2[1:/2/dx-A $-B$;

$\operatorname{rhs}[1]:=u 1[1-1] *((1-e u) * u 1[1] / 2 / d x-A+B)$

$+u 1[1] *(1 / d t+2 * A-1 / d t * D)$

$+u 1[1+1] *(-(1-e u) * 1: 1[1] / 2 / d x-A-B)$

- F:

end;

TRIDIAG (diagl, diag2, diag $3, u 2$, rhs $, 1, n 1)$; end;

Procedure MOMENTUM;

begin

if $t<=$ endmotion tnen MOHENTUM_Fxt

el se MOHENTUM_FX;

end;

Procedure DRAWBED(vivector);

$\operatorname{var} x 1, x 2, y 1, y 2:$ real;

begin

$H_{1}$ Res;

GraphW1ndow $(50,40,589,159)$;

GoToxy $(35,20)$; writeln ('t $=$ ', t:5:3);

for $1:=0$ to $n 1$ do

begin

$\times 1:=(1-1) /(n 1) \div 540$;

$y 1:=\vee[1] * 30$;

$\times 2:=1 /(n 1) * 540$

$y 2:=v[1+1] * 30$;

$\operatorname{draw}(r$ ound $(x 1), 60+$ round $(y 1)$, round $(x 2), 60+r$ ound $(y 2), 1)$; end;

end ; 
Procedure DRAUSURFACE(v: vector);

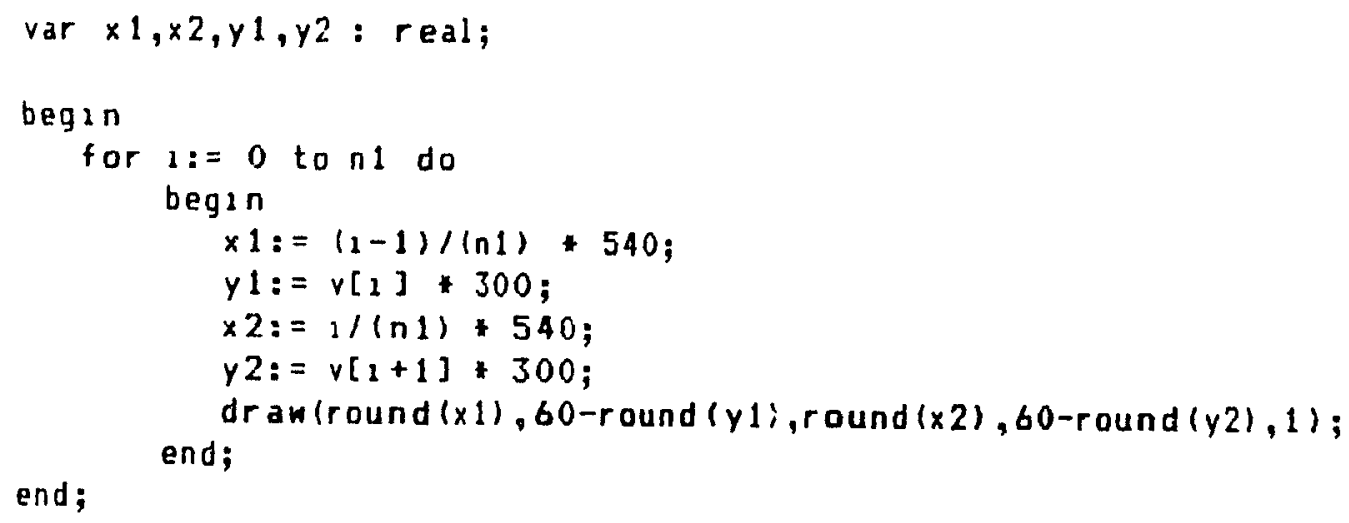




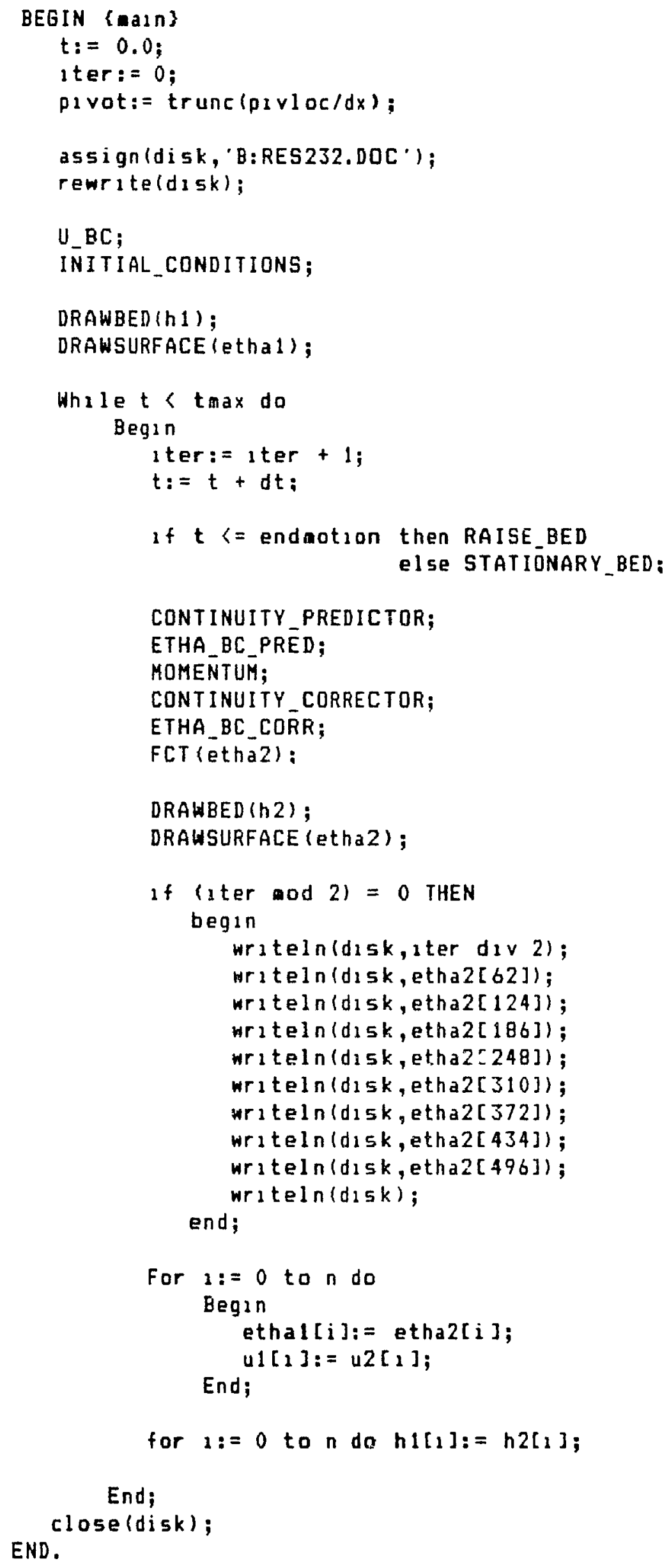




\title{
Program WEDGE
}

\section{Purpose of the program}

Modelling of nonlinear, dispersive, shallow-water waves generated in a channel by a moving inclined wall (moving wegde).

\section{Definition of the constants}

\author{
$n$ : Total number of $5 p a t a l$ grad points $(1=0,1, \ldots n)$. \\ $d x$ : Initial spatial mesh slze at $t=0$. \\ dt : Temporal mesh size. \\ totald: Total length of the channel. \\ thax : Total duration of the simulation. \\ vstar : Amolitude of the sinusoidal wall velocity. \\ slope : Constant slope of the moving wedge. \\ last T: Time at which the motion of the wedge stops. \\ lastx : Final : -coordinate of the moving wall. \\ smooth : Farameter controlling the lenght of the smoothed transitior \\ between the unclined wall and the channel bottom. \\ $91, \ldots, g^{8}$ : Location of the ezght qauges measuring wave amplitude.
}

\section{Definition of the global variables}

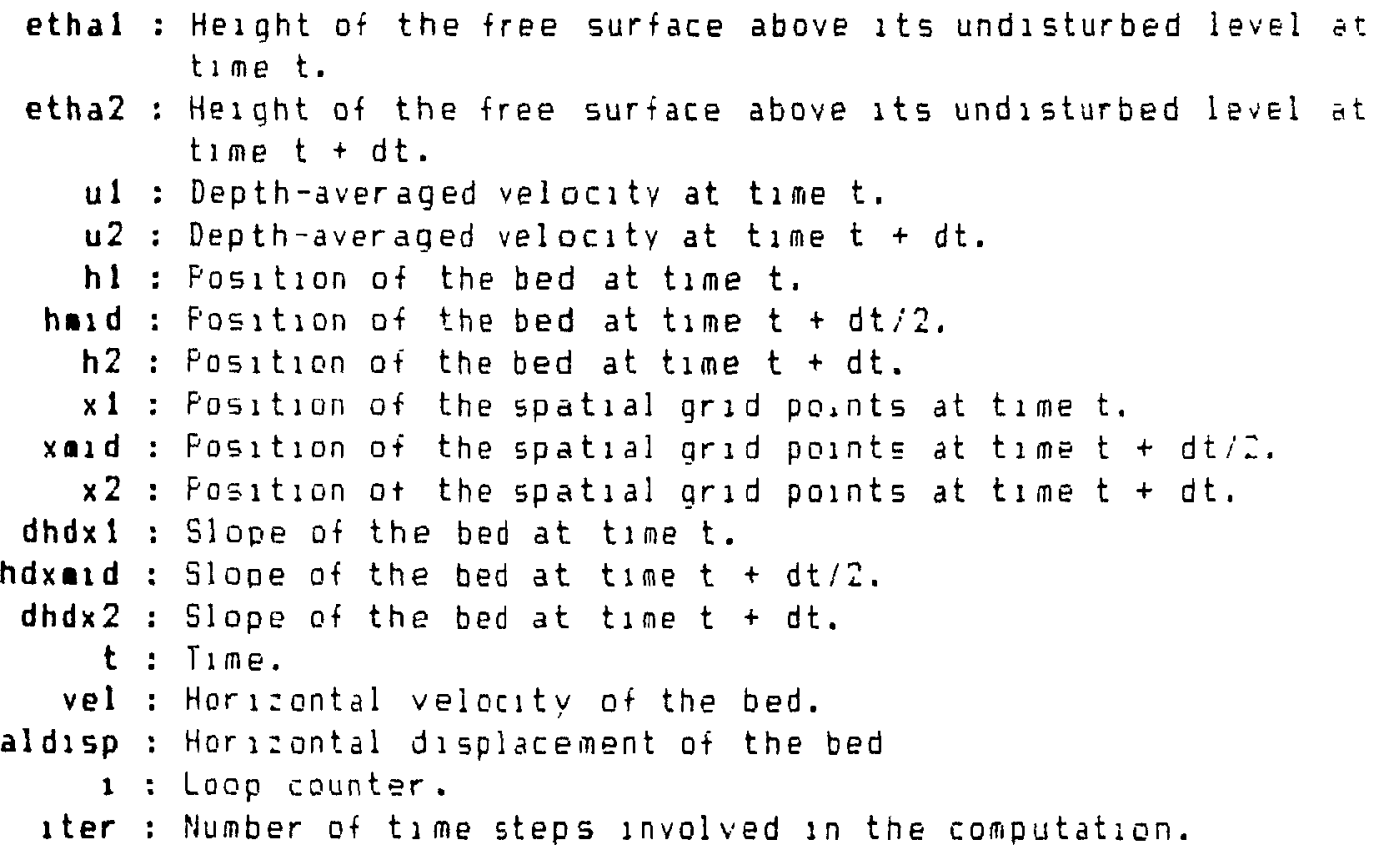


gaugel...

gauge8 : Grad points corresponding to the gauges location.

disk : Te:t file used to store the results.

\section{Program listing}

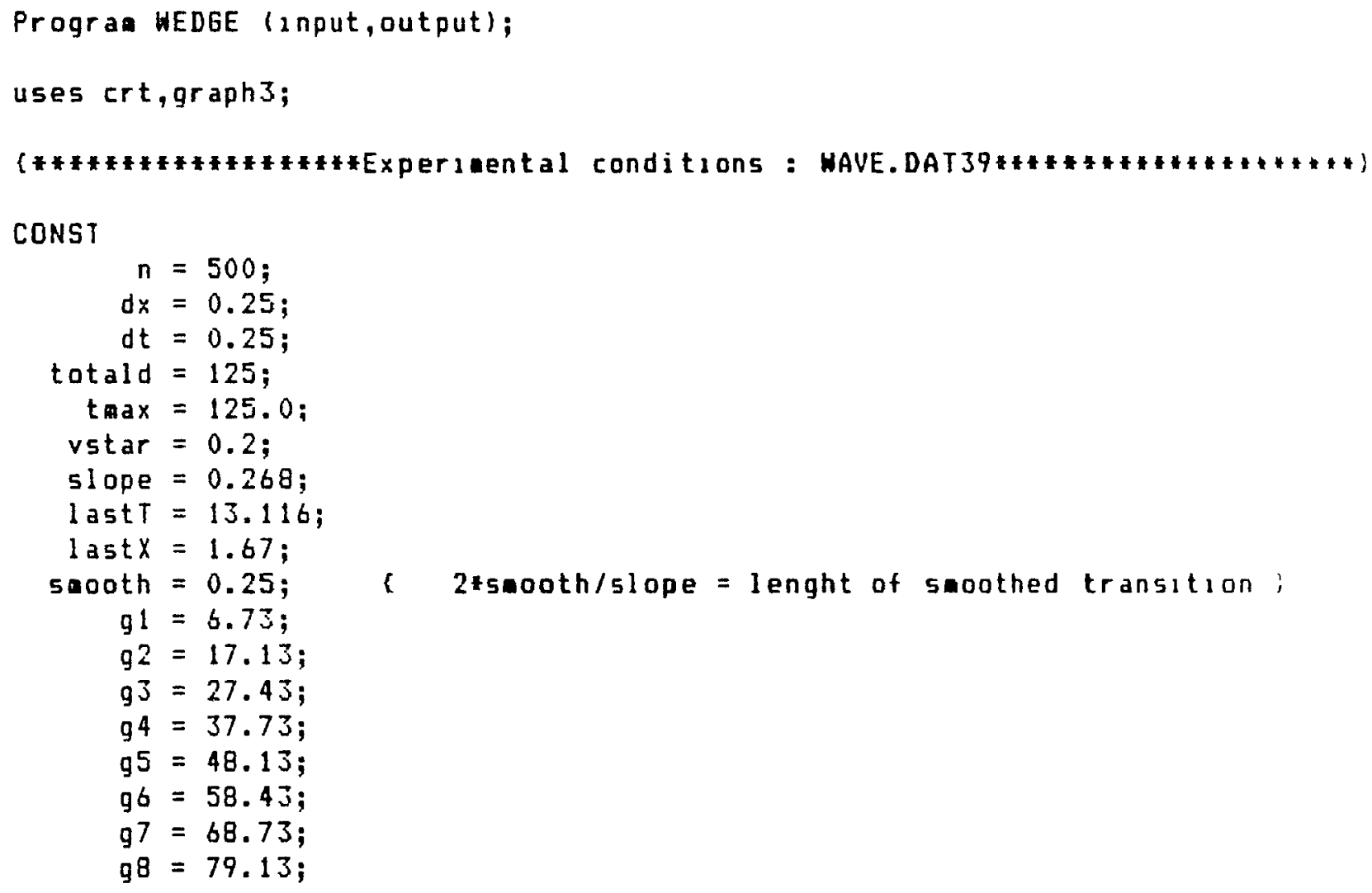




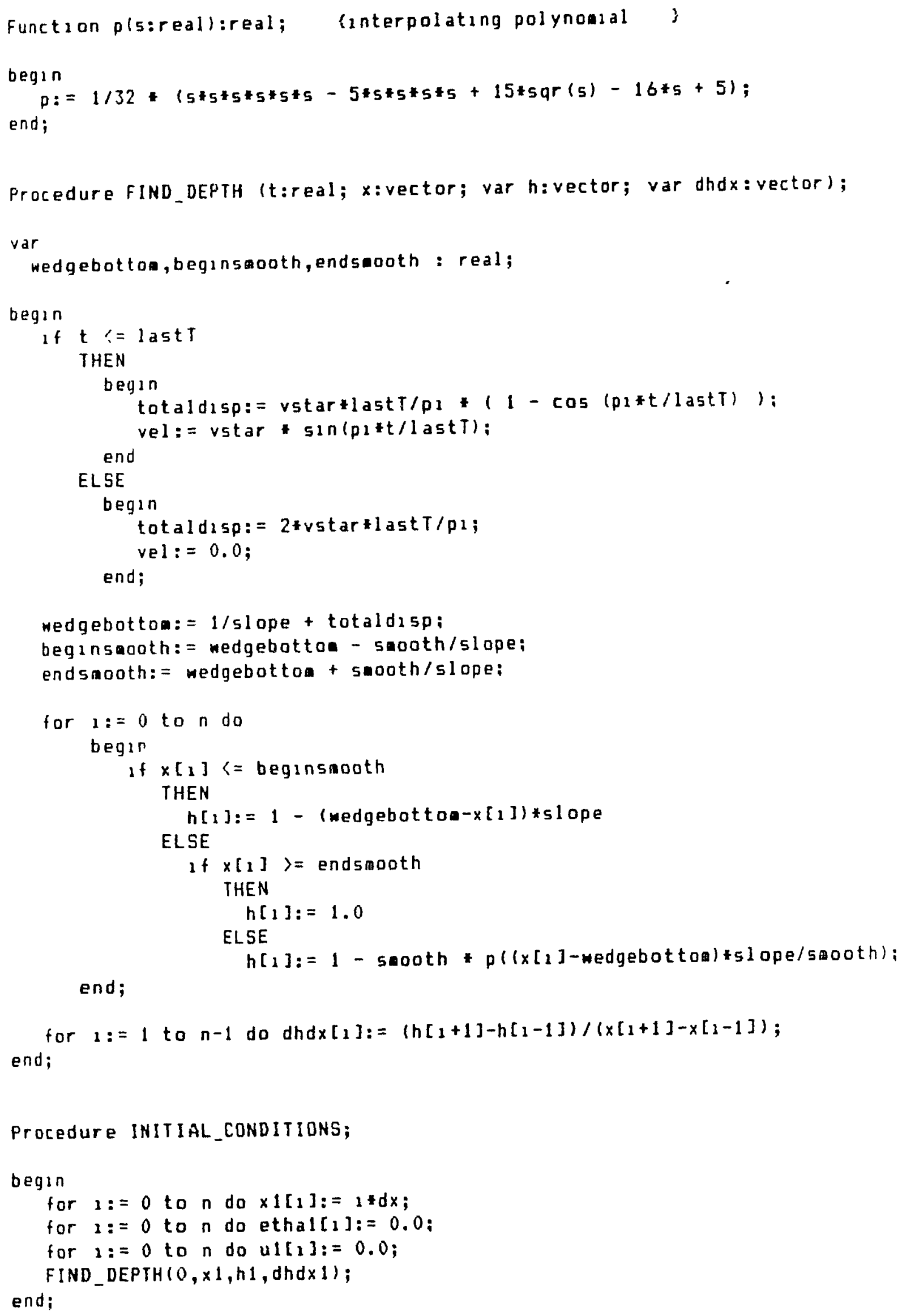


Procedure APPROX_INTERFACES:

begin

for $1:=0$ to $n$ do

begin

$x \operatorname{ad}[1]:=x 1[1]+d t / 2 * u 1[1]$;

$\times 2[1]:=x 1[1]+d t * u[[1]$ :

end:

end;

Procedure CONTINUITY_PREDICTOR;

begin

for $3:=1$ to $n-1$ do

begin

etha2[1]:= etha1[1] $-(h 2[1]-h 1[2])$

- dt * (ethal[1]+hi[1])

- $(u 1[1+1]-u 1[1-1]) /(x|[1+1]-x|[1-1])$ :

end;

end;

Procedure CONTINUITY_CORRECTOR;

begin

for $1:=1$ to $n-1$ do

begin

etha2[1]:= ethal[1] - (h2[1]-h1[1])

- dt $* 0.5 *($ etha $1[1]+\operatorname{etha} 2[1]+h 1[1]+h 2[1])$

* $0.5 *($ (u) $[1+1]-u([1-1]) /(x)[1+1]-\times 1[1-1])$

$+(u 2[1+1]-u 2(1-1]) /(\times 2[1+1]-\times 2(1-1])) ;$

end;

end;

Procedure FS_BC;

begin

etha2[0]:= - h2[0];

etha $2[n]:=$ etha $2[n-1]$;

end;

Procedure U_BC_PRED;

begin

$u 2[0]:=u 1[0]$;

$u 2[n]:=0$;

end; 


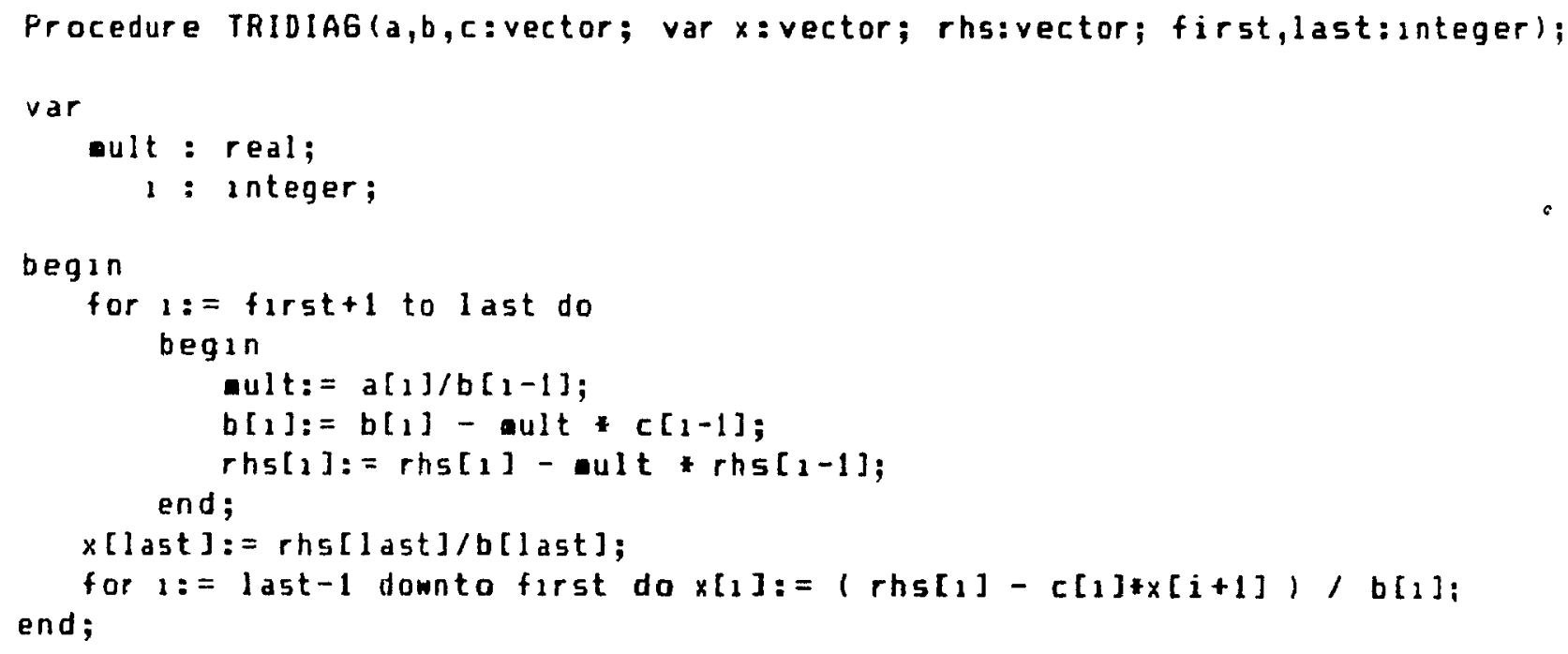




\section{ELSE}

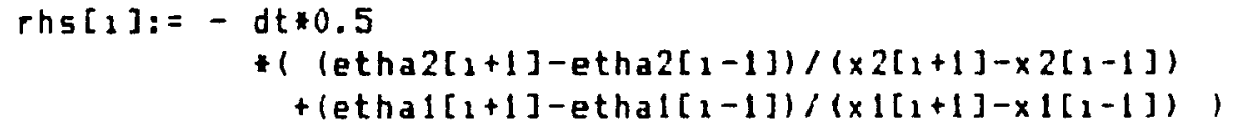

Procedure INTERFACES;

begin

$\times 2[0]:=\times 1[0]+0.5 * d t *(u 1[0]+u 2[0]) ;$

for $1:=1$ to $n$ do

begin

$x 2[1]:=x[1]+0.5 * d t *(u)[i]+u 2[1]) ;$

If $(\times 2[1-1]<=g 1)$ and $(\times 2[1]>g 1)$

then $1 f(g 1-x 2[1-1])<=(\times 2[1]-g l)$ then gauge $1:=1-1$

else gauge $1:=1$;

If $(\times 2[1-1]<=g 2)$ and $(\times 2[2]>g 2)$

then if $(g 2-x 2[1-1])(=(\times 2[1]-g 2)$ then gauge $2:=1-1$

if $(\times 2[1-1]<=g 3)$ and $(\times 2[1]>g 3)$

el se gauge $2:=1$;

then if $(93-\times 2[1-1])<=(\times 2[1]-g 3)$ then gauge $3:=1-1$

else gauge $3:=1$;

if $(\times 2[2-1]<=94)$ and $(\times 2[1]>g 4)$

then if $(94-x 2[3-1])<=(\times 2[1]-94)$ then gauge $4:=1-1$

else gauge $4:=1$;

if $(\times 2[1-1]<=95)$ and $(\times 2[2]>g 5)$

then if $(95-\times 2[1-1])<=(\times 2[1]-g 5)$ then gauges: $=1-1$

else gauge $5:=1$;

if $(x 2[1-1](=96)$ and $(x 2[1]>96)$

then if $(96-\times 2[1-1])<=(\times 2[1]-96)$ then gaugeb: $=1-1$

else gaugeb: $=1$;

if $(\times 2[1-1]<=g 7)$ and $(\times 2[1]>97)$

then if $\{g 7-x 2[1-1]\rangle<=\langle\times 2[1]-g\rangle)$ then gauge $7:=1-1$

el se gauge $7:=1$;

If $(x 2[1-1]<=98)$ and $(\times 2[1]>g 8)$

then if $(98-\times 2[1-1])<=(\times 2[1]-98)$ then gauge $8:=1-1$

end;

el se gauge8: = 1;

end; 


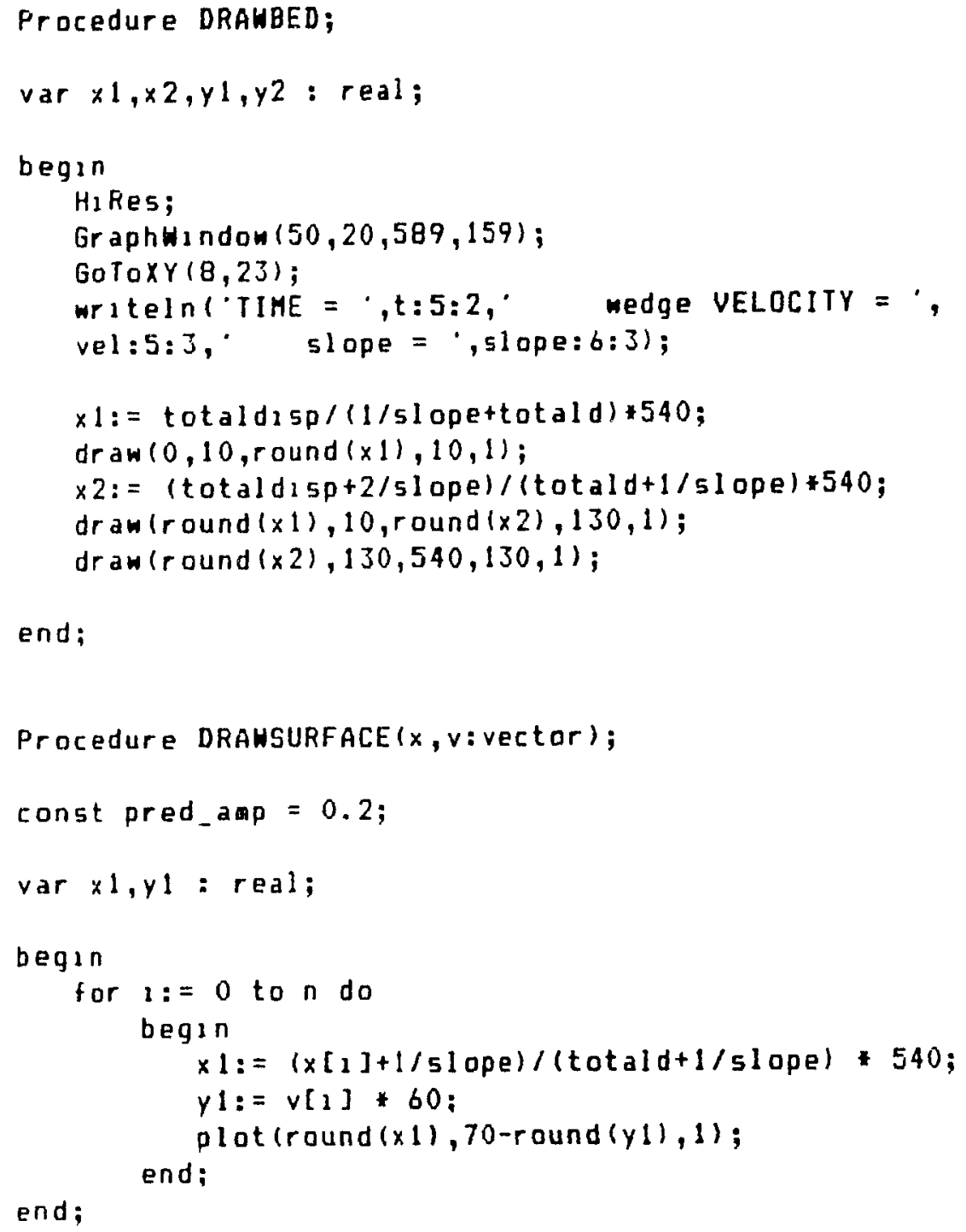




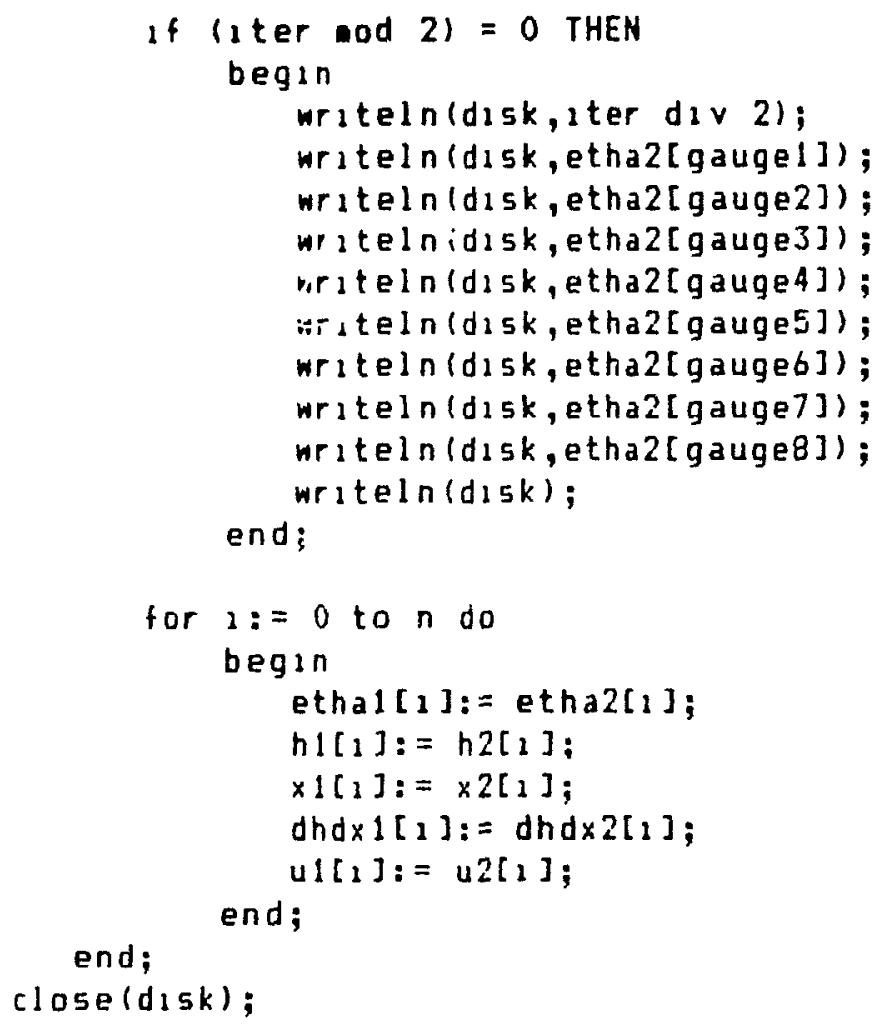


APPENDIX D

Supplementary results 


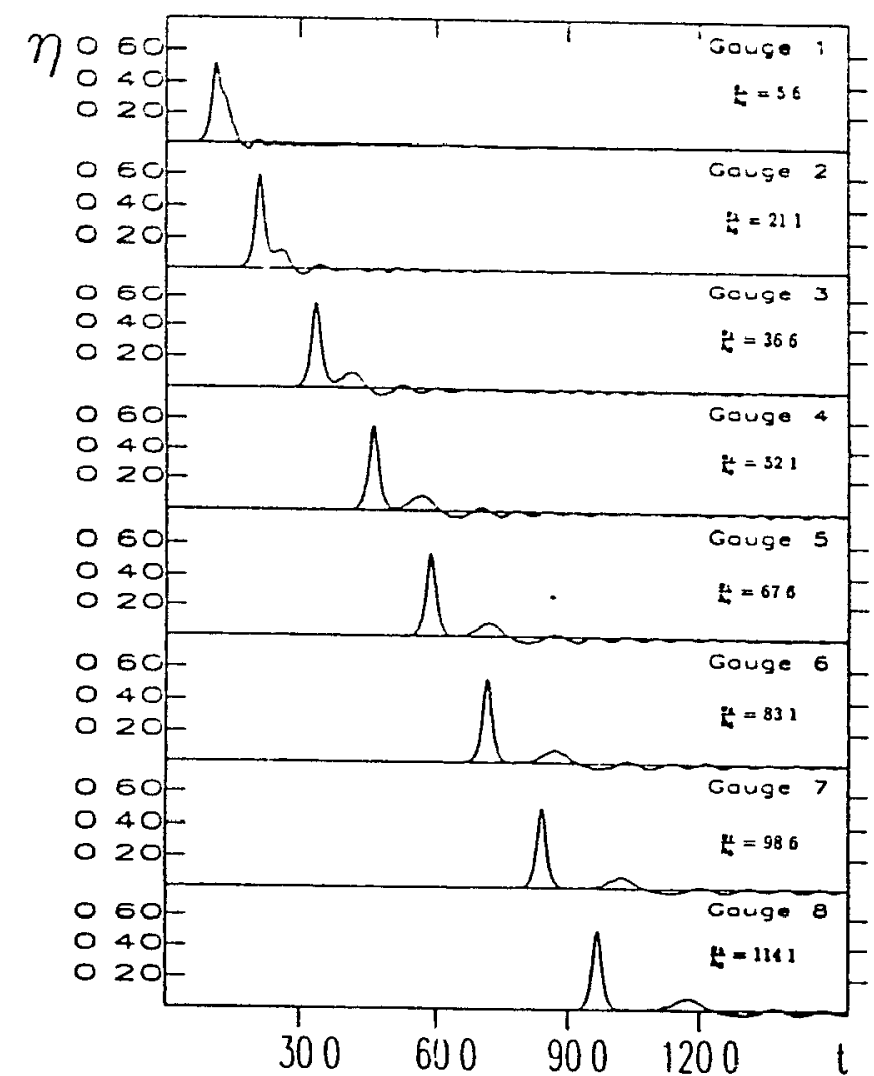

a) Experiment

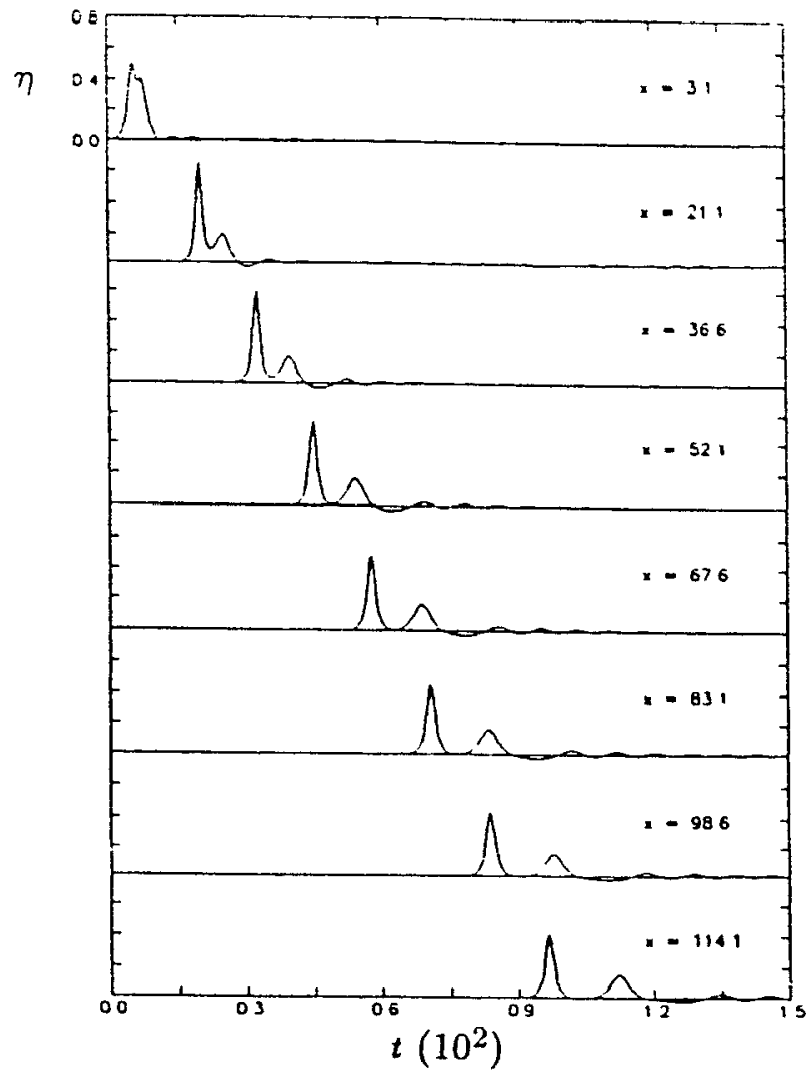

b) Computation

Figure D.1: Wave generated by a moving wall. Maximum horizontal velocity of the wall $v^{*}=$ 0.4 ; total displacement $d=2.5$; duration of the motion $t_{f}=9.82$. Note : The trme coordlnate corresponding to $t=0$ may slightly differ in the computations and experiments. 


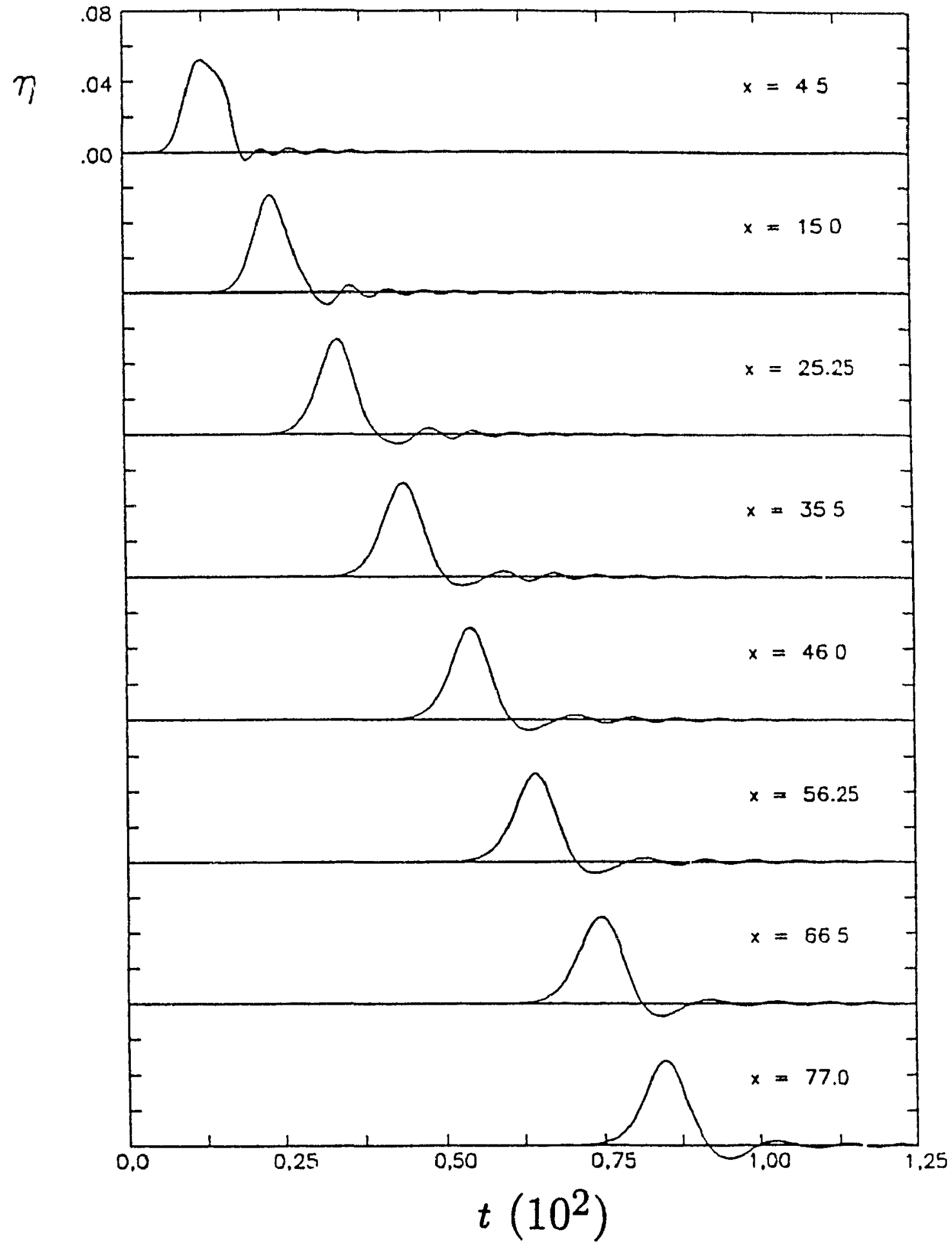

Figure D.2a: Wave generated by a submerged wedge. Slope $m=0.268$;maximum horizontal velocity of the wedge $v^{*}=0.2$; total displacement $d=1.67$; duration of the bed motion $t_{f}=13.12$. 


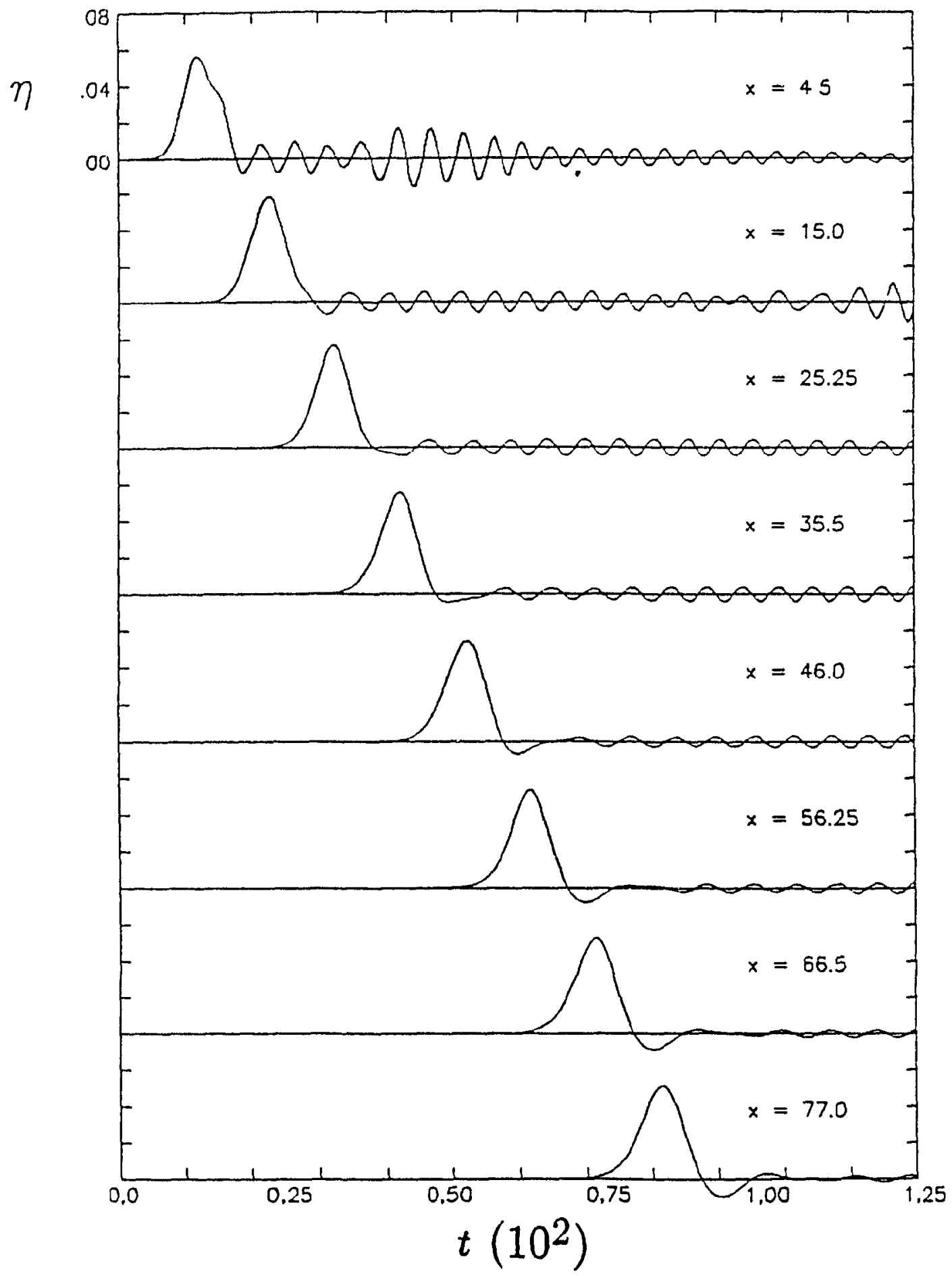

Figure D.2b: Wave generated by a submerged wedge. Results obtained without the use of the FCT algorithm. 


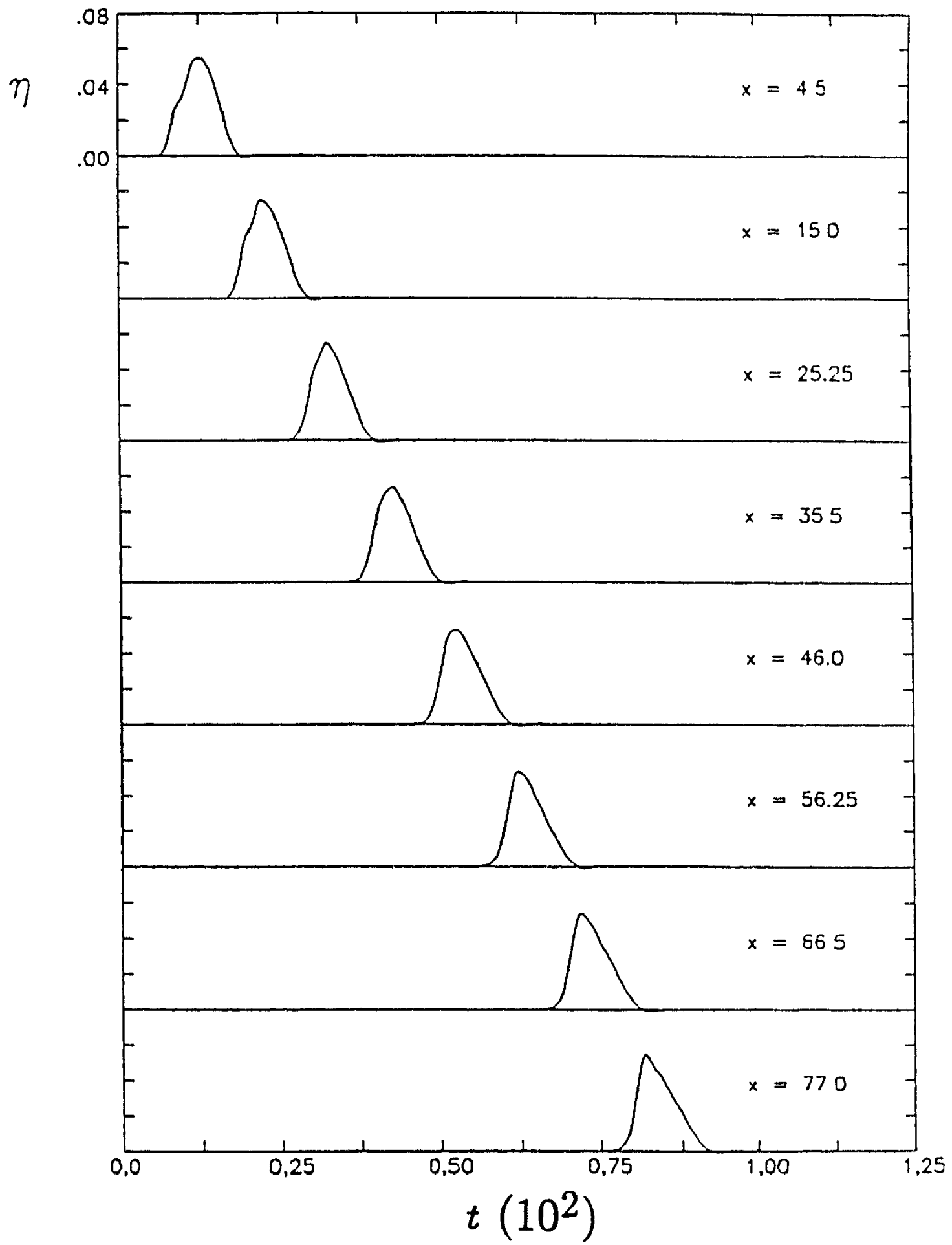

Figure D.2c: Wave generated by a submerged wedge. Cornputations based on the: linearized long-wave theory. 


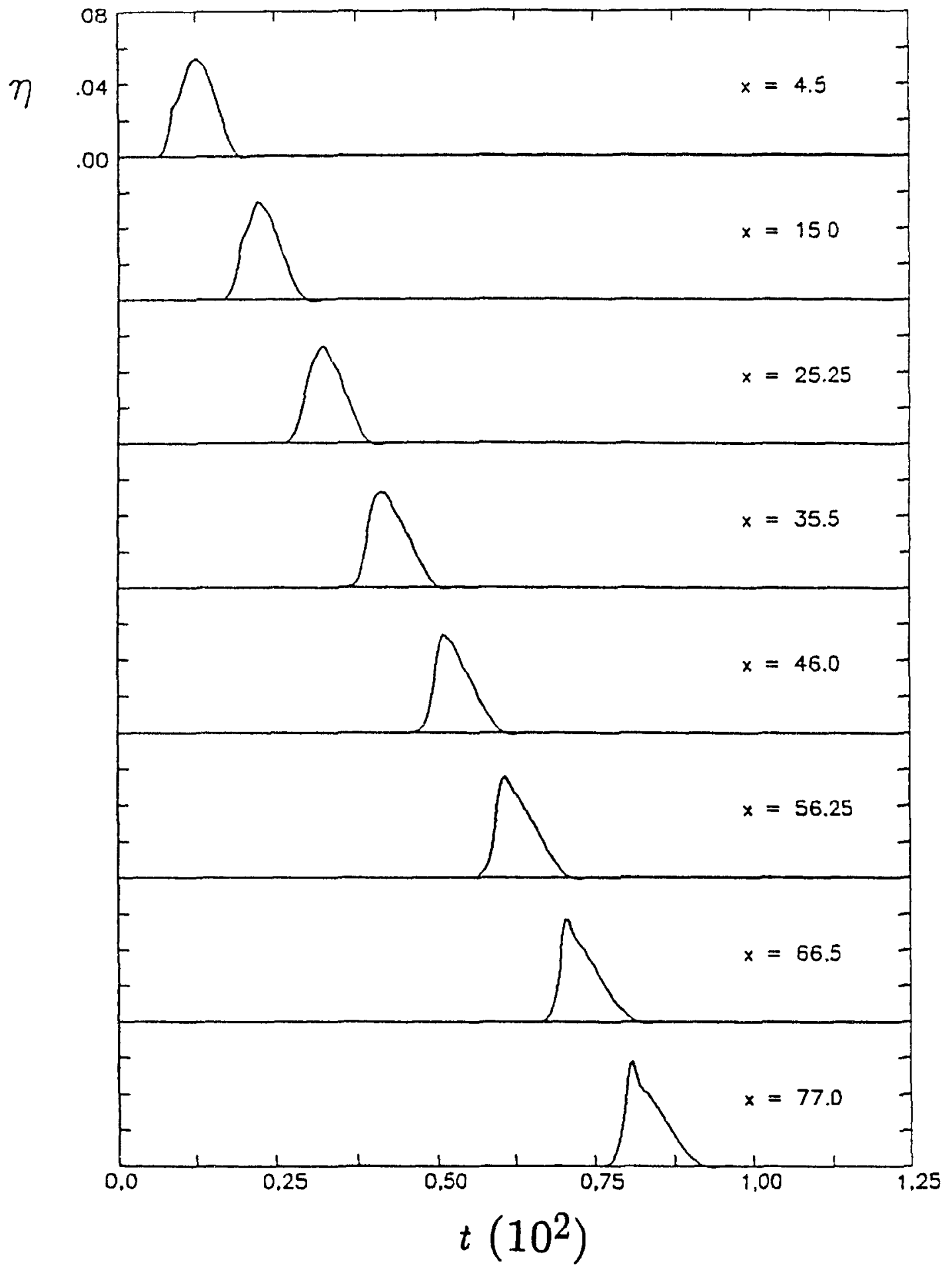

Figure D.2d: Wave generated by a submerged wedge. Computations based on the shallow-water, finite-amplitude theory. 


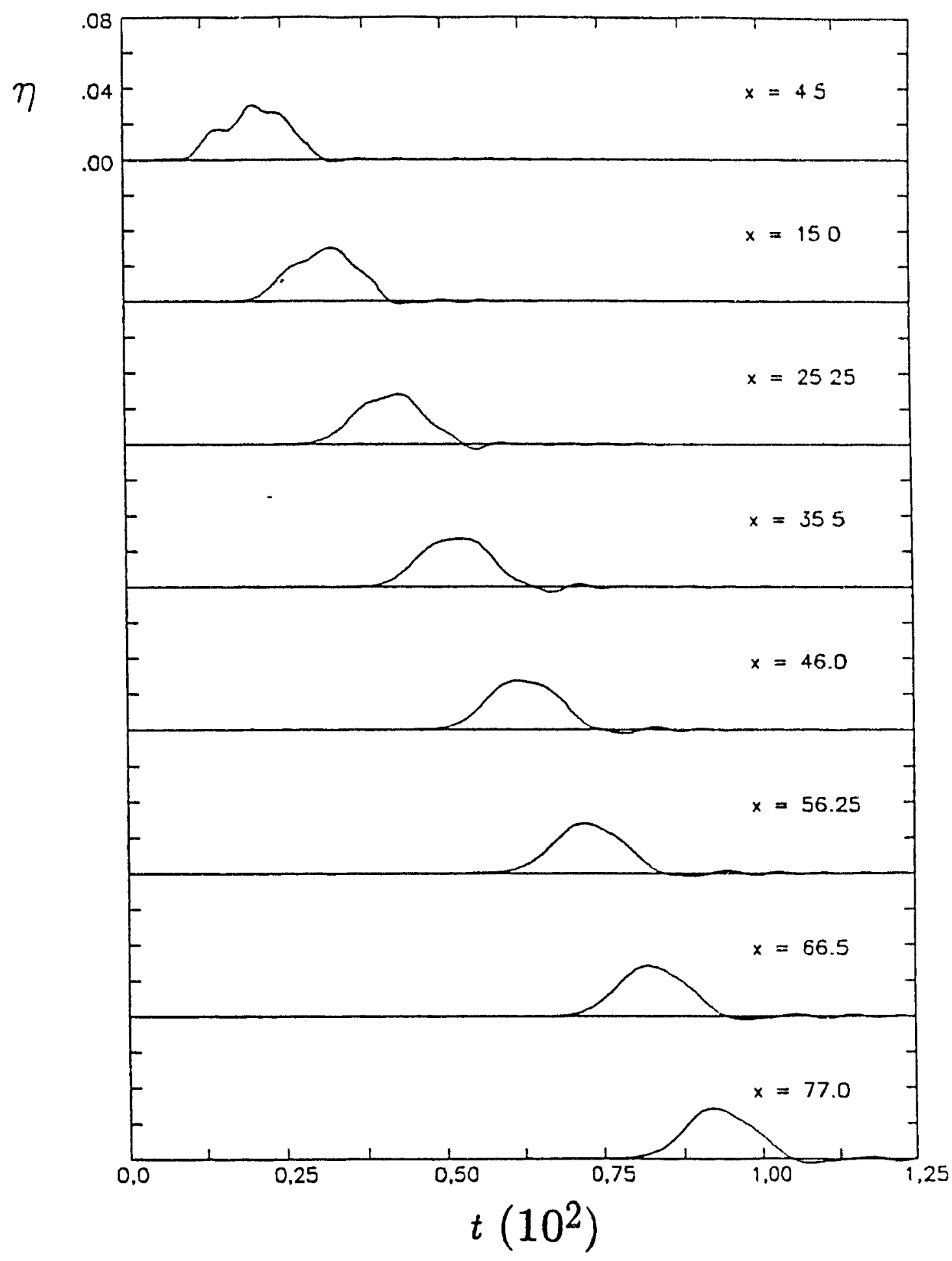

Figure D.2e: Wave generated by a submerged wedge. Slope $m=0.268$;maxmum horizontal velocity of the wedge $v^{*}=0.1$; total displacement $d=1.67$; duration of the bed motion $t_{f}=26.23$. 


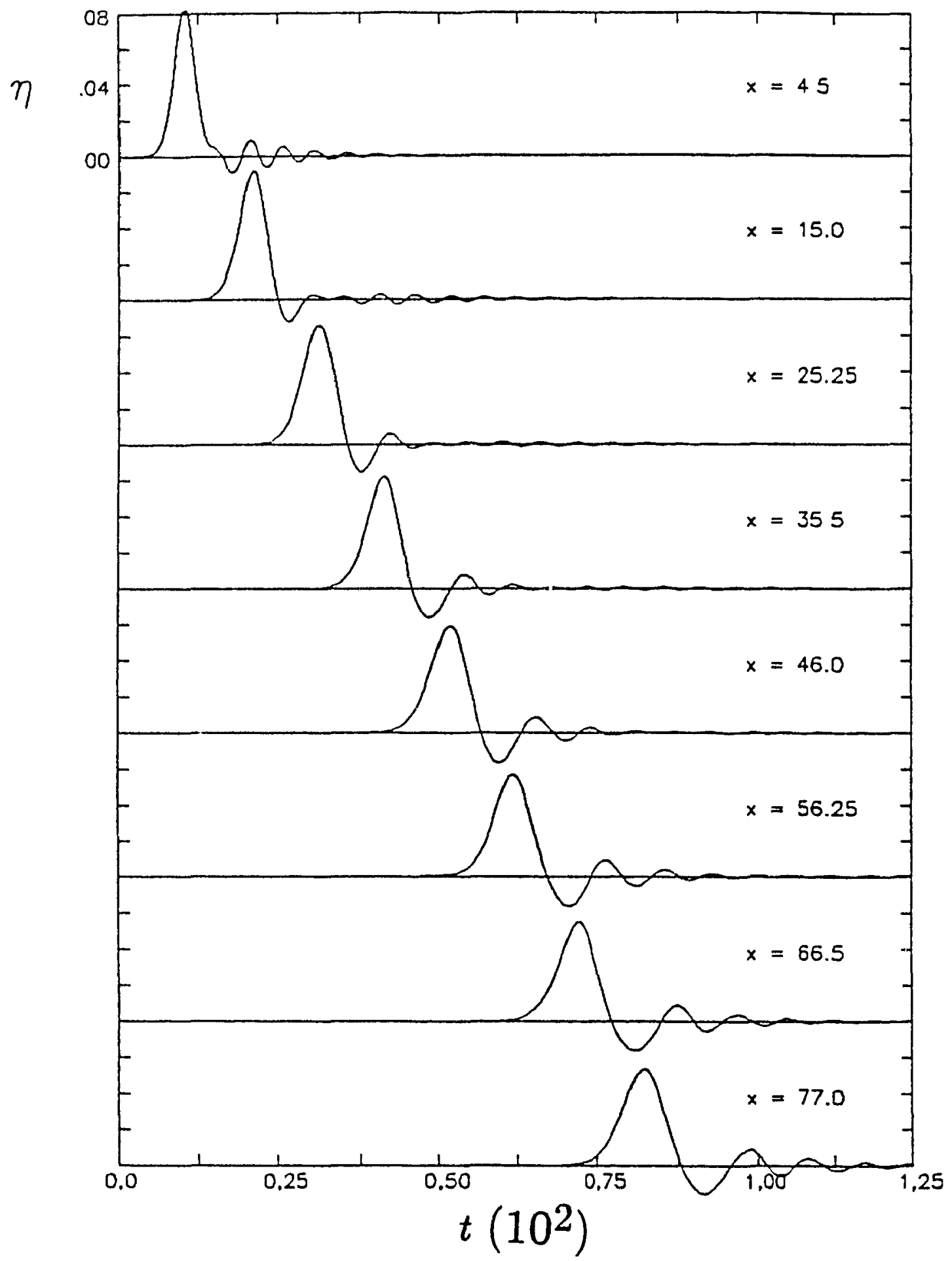

Figure D.2f: Wave generated by a submerged wedge. Slope $m=0.577 ;$ maximum horizontal velocity of the wedge $v^{*}=0.2$; total displacement $d=1.14$; duration of the bed motion $t_{f}=8.94$. 


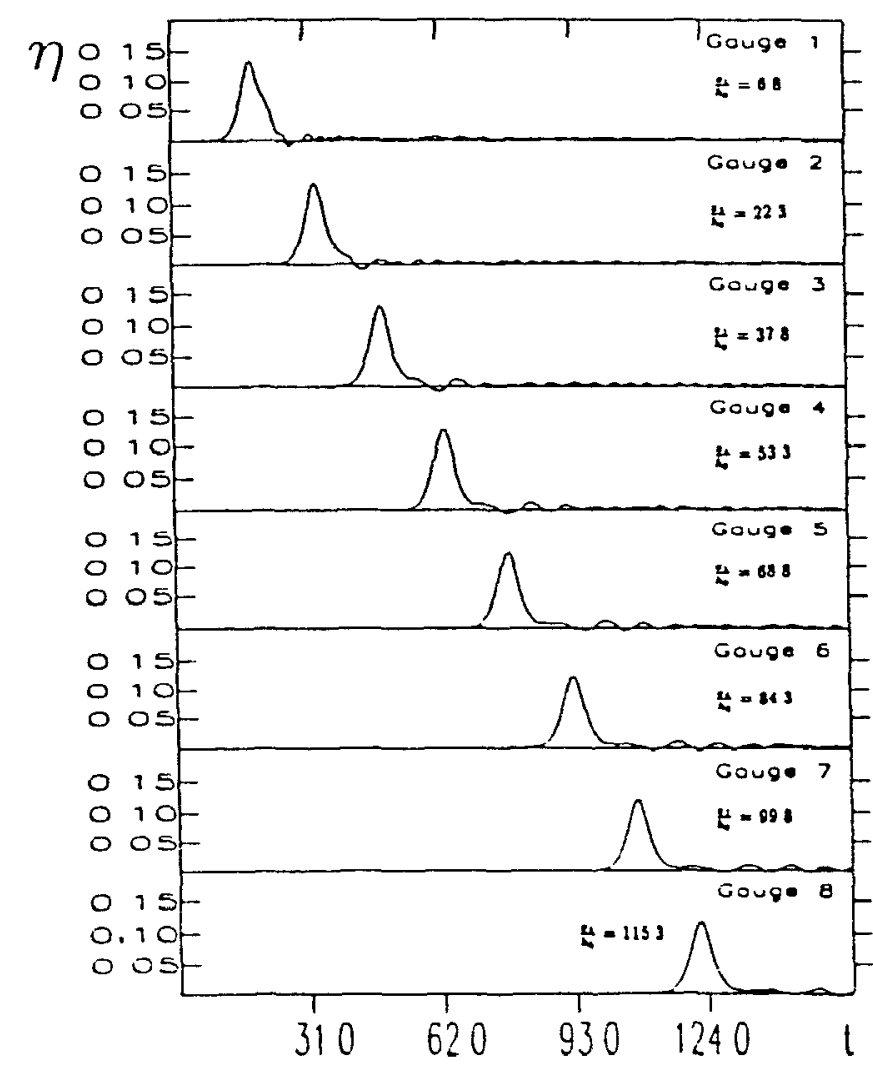

a) Experiment

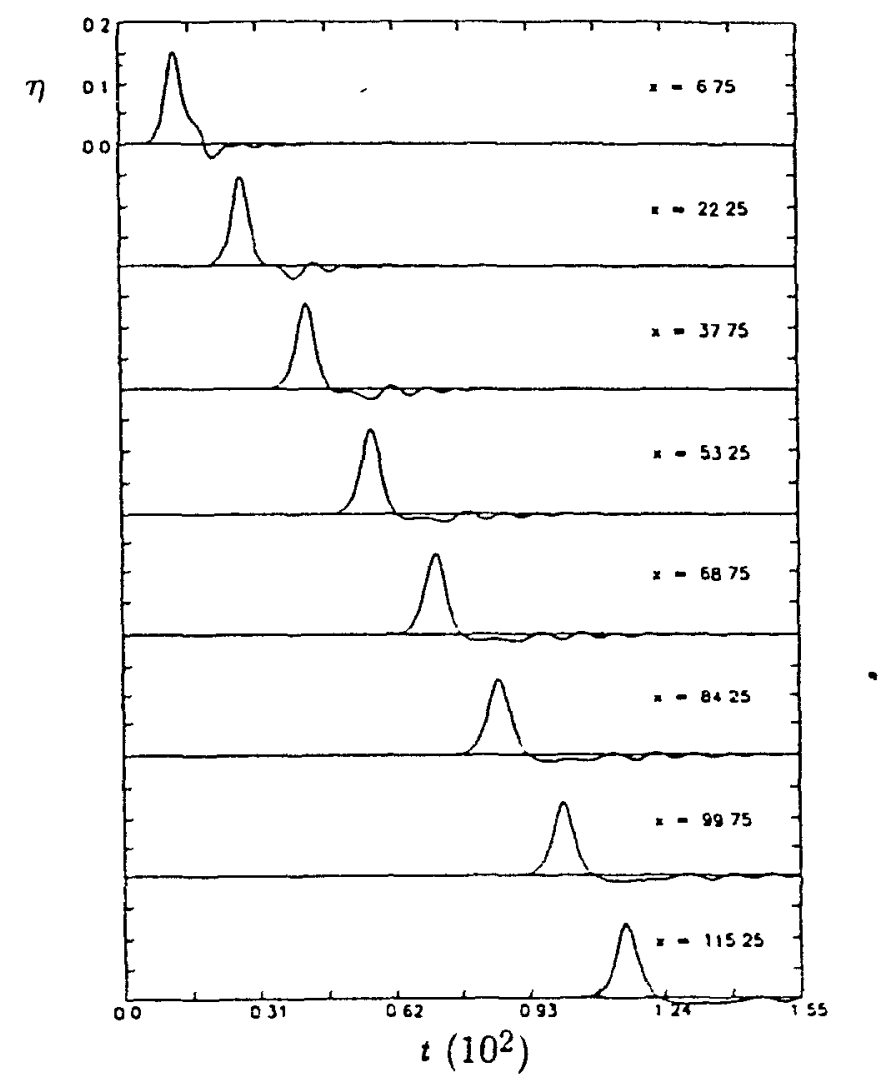

b) Computation

Figure D 2g: Wave generated by a submerged wedge. Slope $m=0.268$; maximum horizontal velocity of the wedge $v^{*}=04$; total displacement $d=25$. duration of the bed motion $t_{f}=9.75$ 


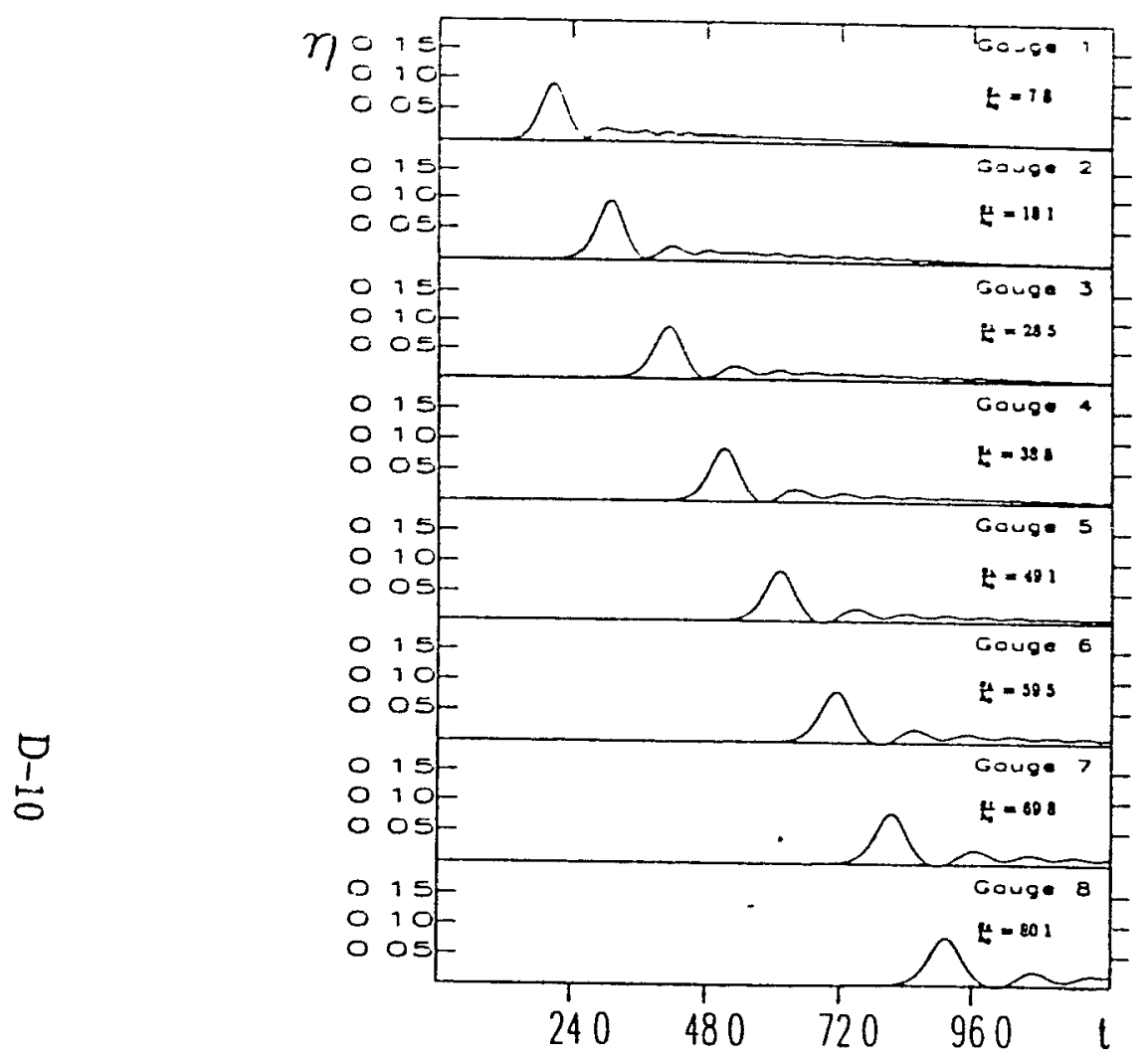

a) Experiment

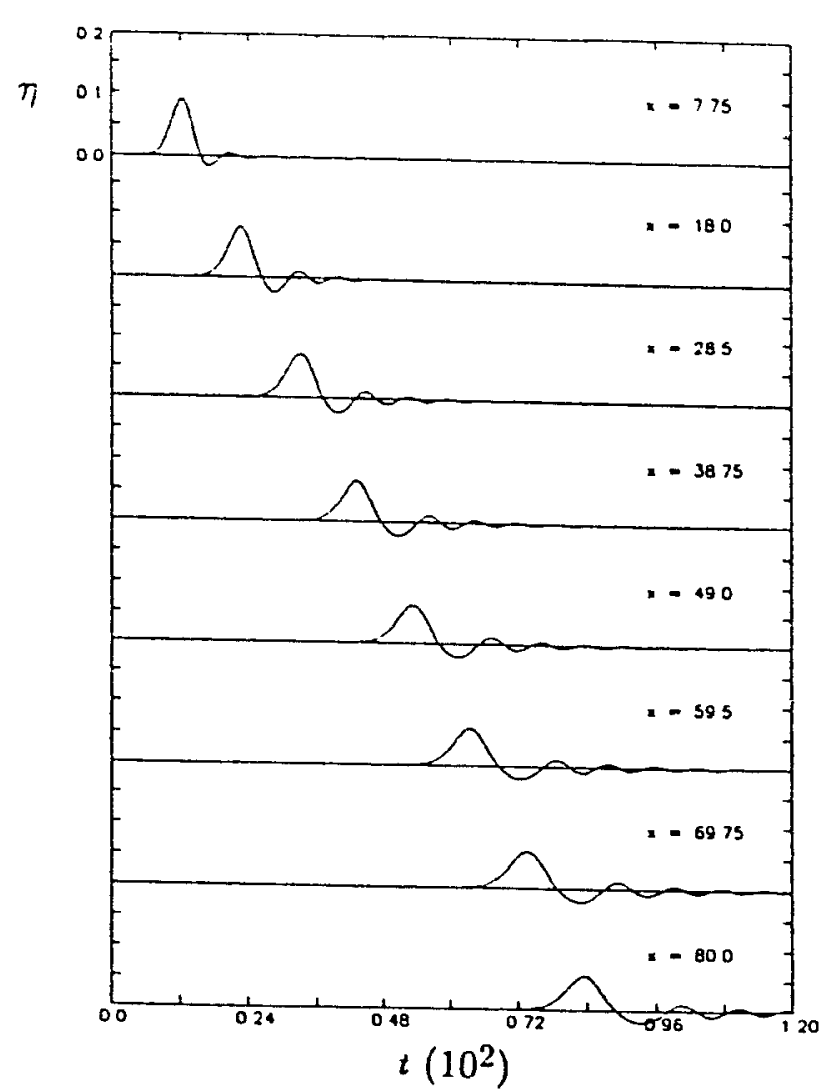

b) Computation

Figure D.2h: Wave generated by a submerged wedge. Slope $m=0.577$; maximum horizontal velocity of the wedge $v^{*}=0.2$; total displacement $d=1.18$; duration of the bed motion $t_{f}=6.18$. 


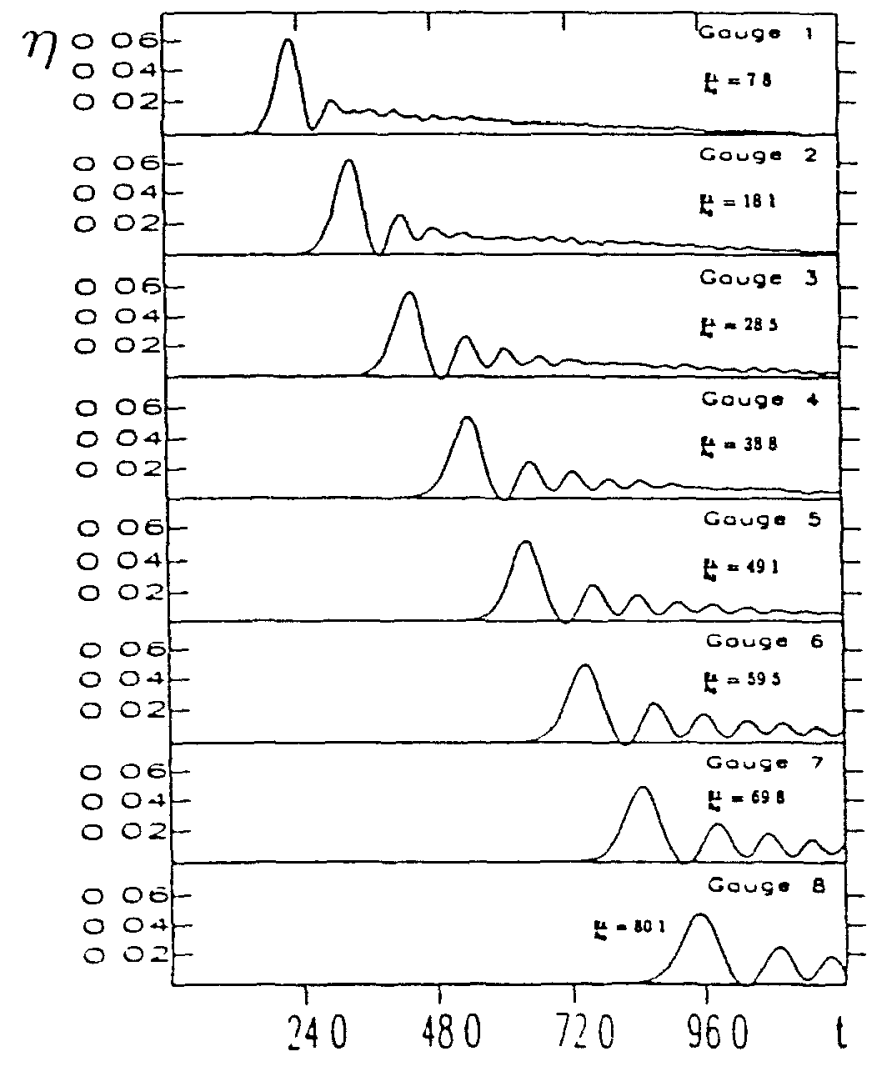

a) Experiment

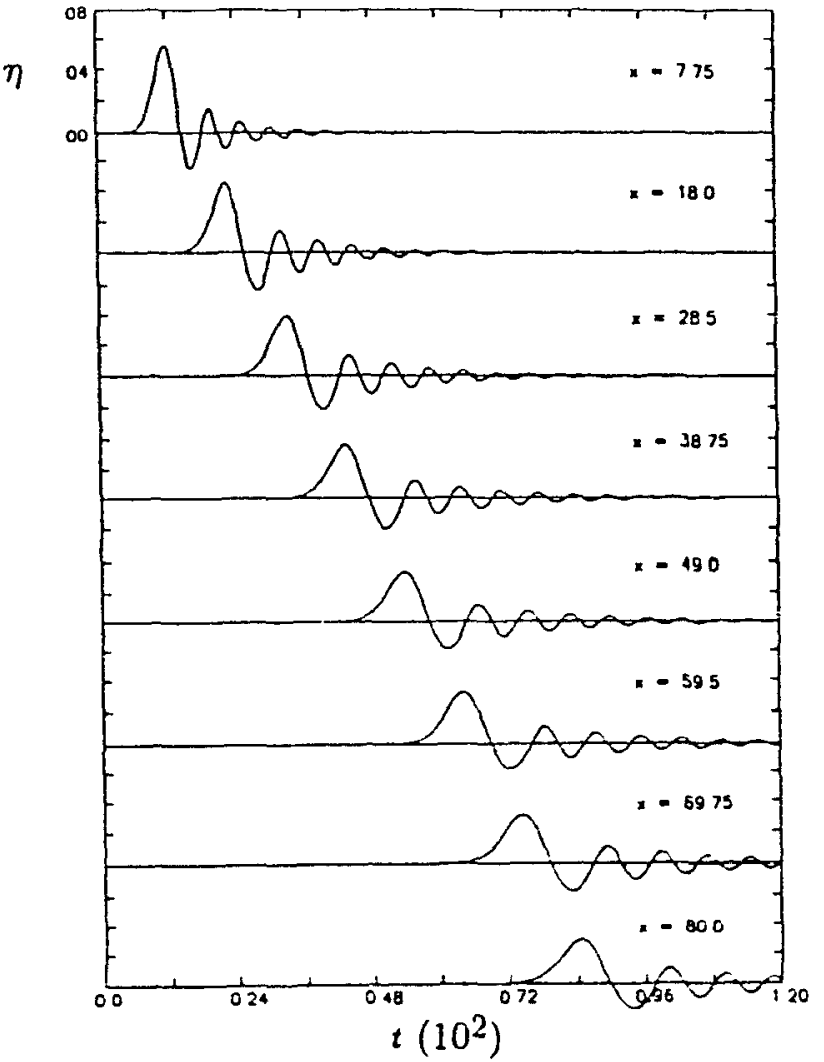

b) Computation

Figure D.2i: Wave generated by a submerged wedge Slope $m=0.577$; maxımum horizontal velocity of the wedge $v^{*}=0.2$; total displacement $d=0.85$, duration of the bed motion $t_{f}=4.75$. 


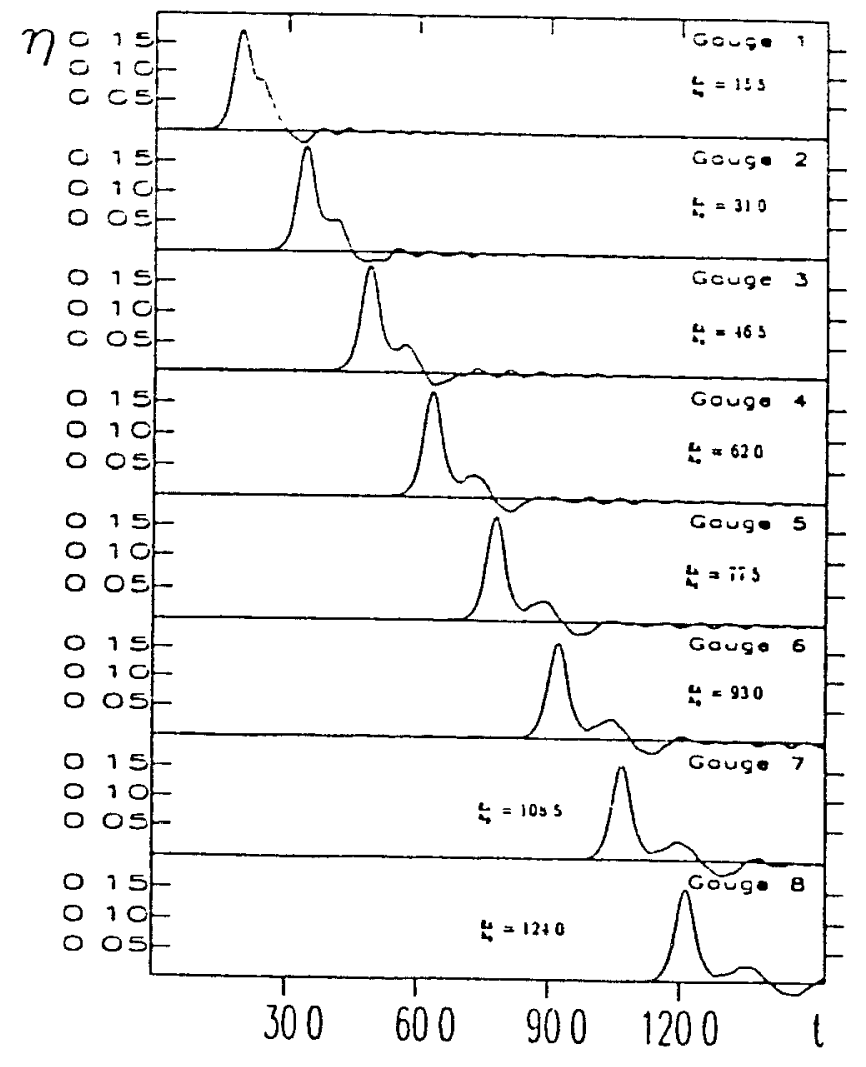

a) Experiment

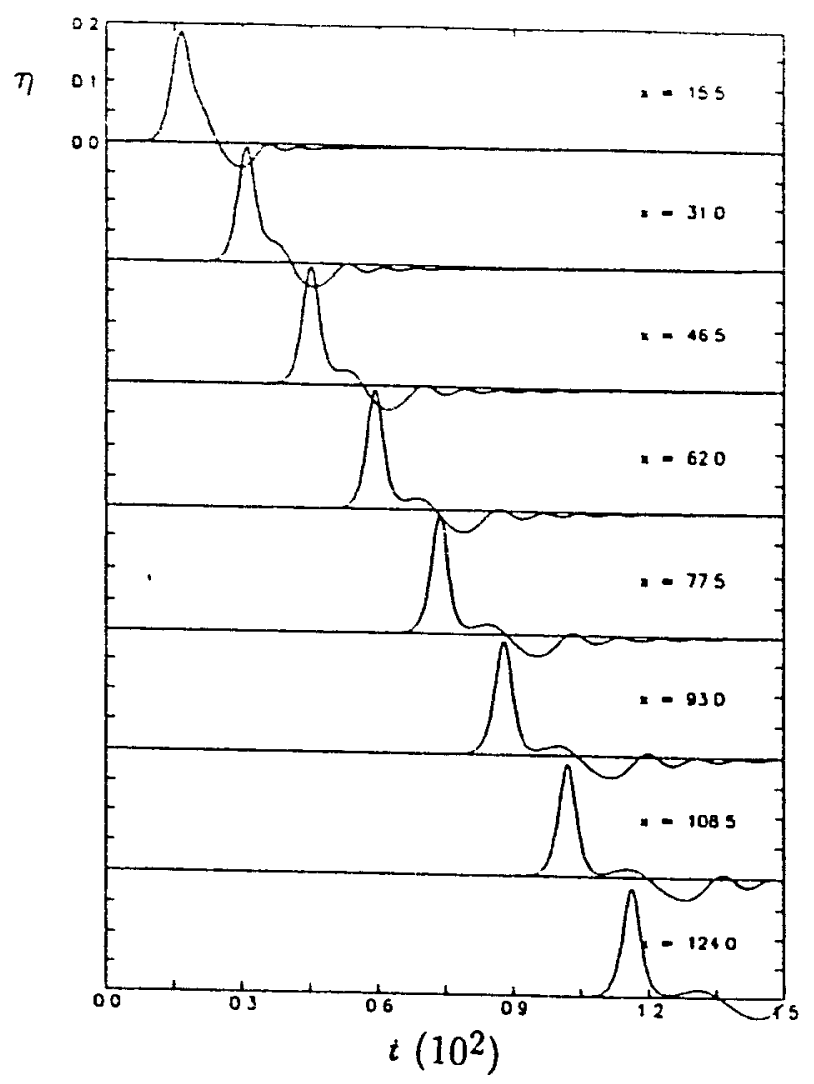

b) Computation

Figure D.3a: Wave generated by a rotating plate. length of the plate $l=5$; vertical pull velocity $V=0.1$ at $x=0$; duration of the bed motion $t_{f}=5.0$; final slope $m_{\max }=0.1$. 


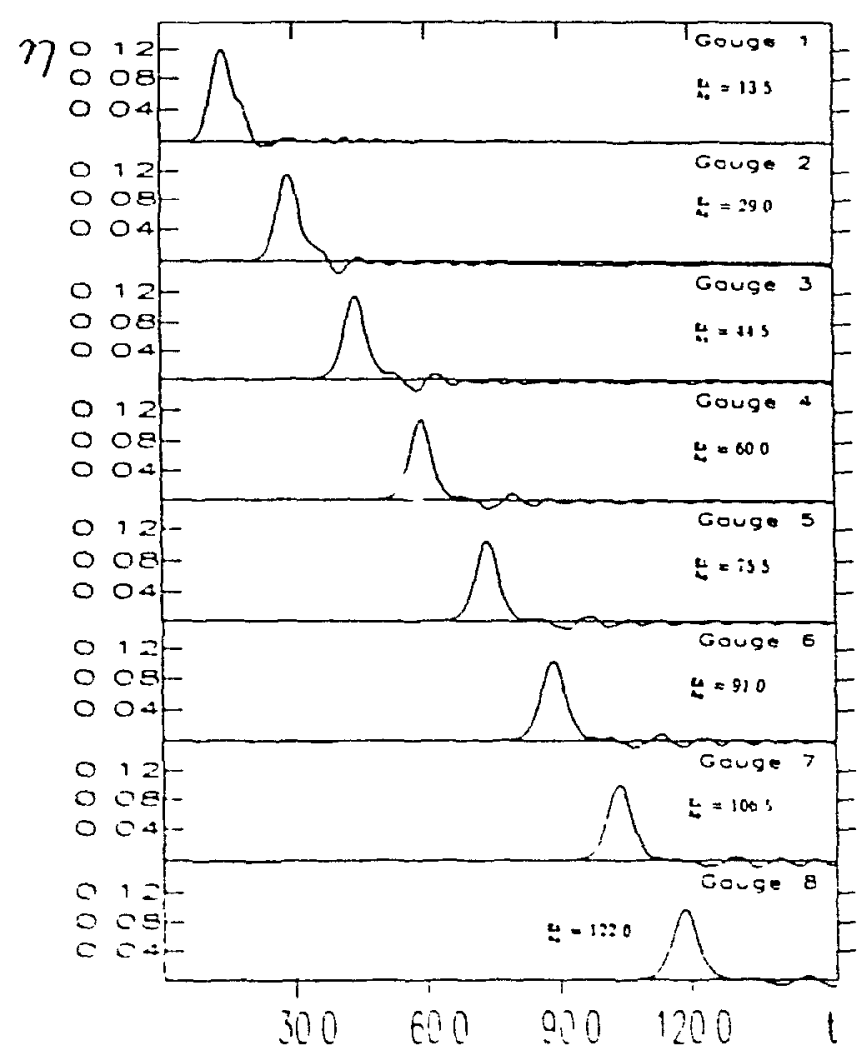

a) Experiment

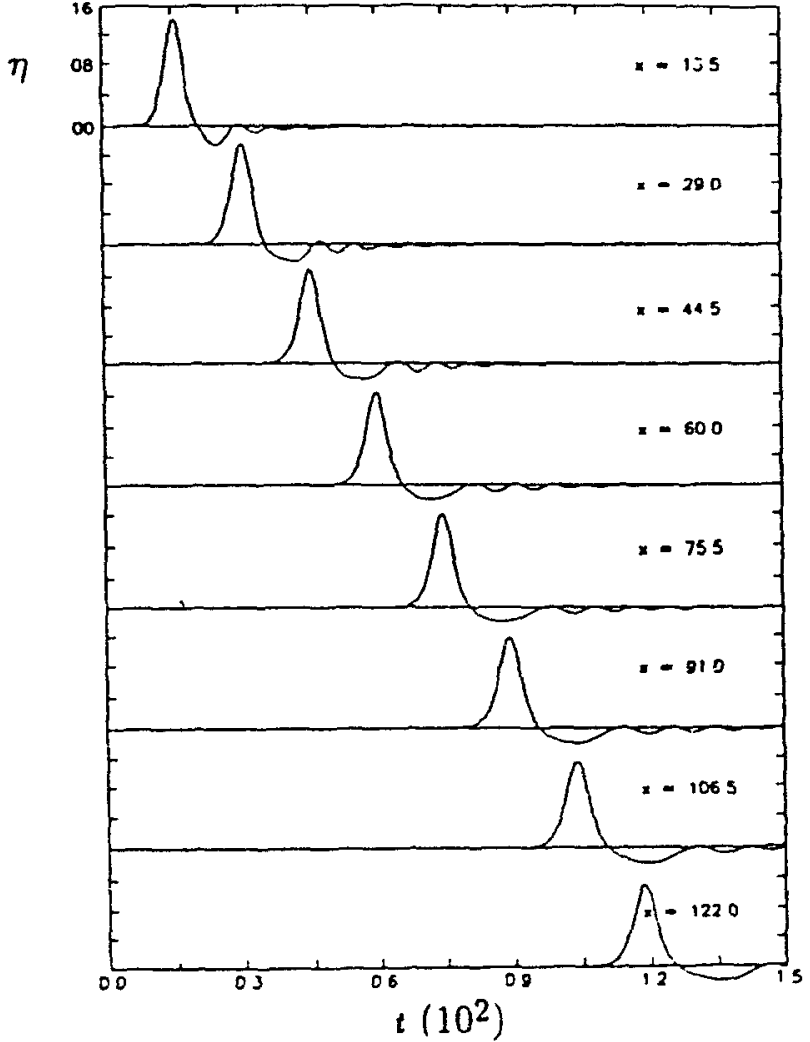

b) Computation

Figure D 3b Wave generated by a rotating plate Length of the plate $l=3$; vertical pull velocity $I^{*}=01$ at $x=0$ : duration of the bed motion $t_{f}=50$; final slope $m_{\max }=\frac{5}{30}$ 


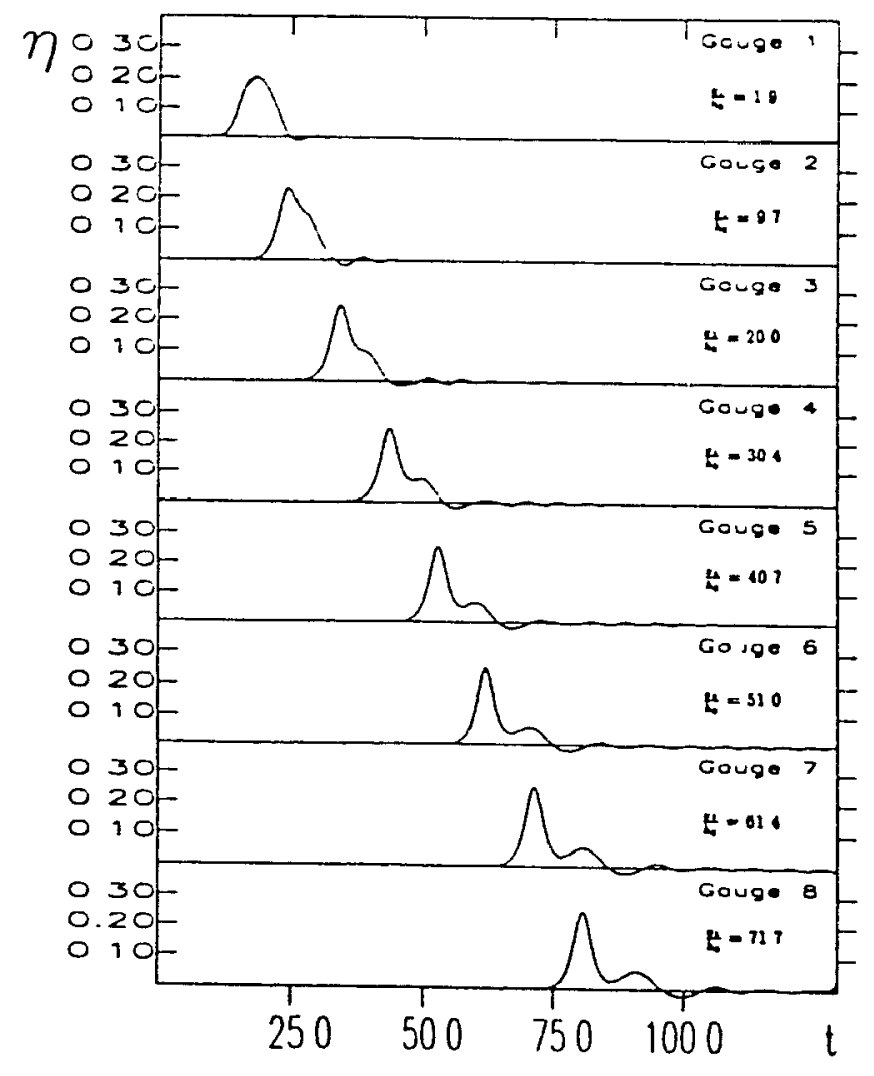

a) Experiment

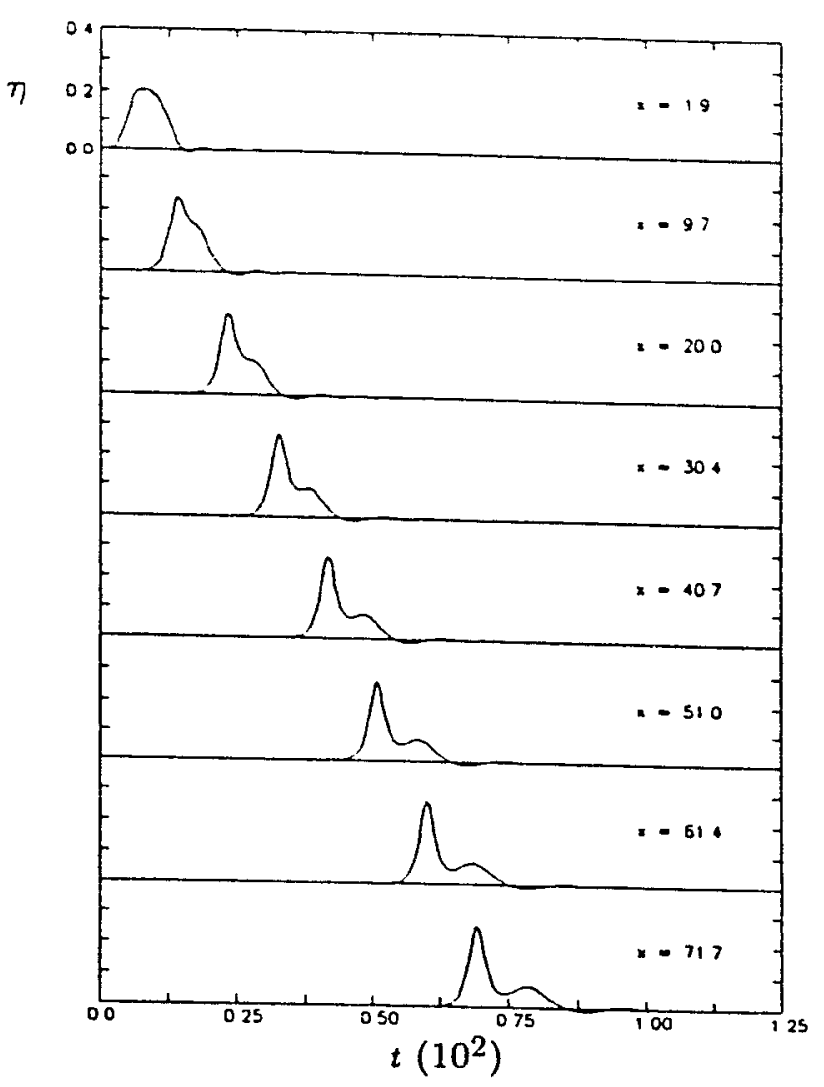

b) Computation

Figure D.4: Wave generated by a moving wedge. Slope $m=1$;maximum horizontal velocity of the wedge $v^{*}=0.2$; total displacement $d=1.67$; duration of the bed motion $t_{f}=13.12$. 